\title{
Relaxation Dynamics in Graphene
}

\author{
Vorgelegt von Master-Physiker \\ Faris Kadi \\ aus Berlin
}

von der Fakultät II - Mathematik und Naturwissenschaften
der Technischen Universität Berlin

zur Erlangung des akademischen Grades

Doktor der Naturwissenschaften

- Dr. rer. nat. -

genehmigte Dissertation

Promotionsausschuss

Vorsitzende: Prof. Dr. rer. nat. Ulrike Woggon, TU Berlin

1. Gutachter: Prof. Dr. rer. nat. Andreas Knorr, TU Berlin

2. Gutachter: Prof. Dr. rer. nat. Felix von Oppen, FU Berlin

3. Gutachter: Prof. Dr. rer. nat. Ermin Malić, Chalmers University of Technology

Tag der wissenschaftlichen Aussprache: 11.09.2015

Berlin 2015 



\section{Abstract}

In this thesis, the relaxation dynamics of nonequilibrium carriers in graphene is investigated. Based on the density matrix approach, the interaction of carriers with light, phonons and electrons is theoretically modelled. The resulting time-, momentum-, and angle-resolved simulation of the carrier dynamics enables the interpretation of recent experimental observations.

Typically, the carrier relaxation has been accessed via high-resolution pump-probe experiments. Common to all studies is a bi-exponential decay of the pump-induced differential transmission (DT) spectrum. The fast decay component in the range of few tens of femtoseconds is assigned to an ultrafast Coulomb-dominated carrier redistribution towards a hot Fermi-Dirac distribution, whereas the slower decay component in the range of a picosecond reflects the equilibration between the electron and the phonon system. However, many studies report a zero-crossing after the initial decay in the transient DT spectrum, where the second decay component characterizes the recovering of the DT signal. In very good agreement with recent pump-probe experiment performed by the group of Prof. Manfred Helm (Helmholtz-Zentrum Dresden-Rossendorf), a microscopic explanation for the occurrence of transient negative differential transmission in graphene is found, where a detailed interplay of intraand interband absorption processes on the transient DT in graphene is investigated. In particular, phonon-assisted intraband processes are shown to lead to an enhanced absorption giving rise to the experimentally observed zero-crossing from positive to negative DT signals.

In collaboration with the group of Prof. Theodore B. Norris (Michigan University, USA), theoretical studies combined with ultrafast time-resolved THz spectroscopy were performed to systematically investigate the hot-carrier dynamics in an array of graphene samples. The theory calculates explicitly the time-dependent response of the system to a THz probe pulse. The calculations reveal that the observed dynamics can be accounted for qualitatively without including any fitting parameters, phenomenological models or extrinsic effects. Specifically, the hot-carrier dynamics are governed by the coupling of extraordinarily efficient carrier-carrier and carrier-phonon interactions. Furthermore, the theory demonstrates that the simple Drude model is insufficient to fully account for the $\mathrm{THz}$ interactions.

Beside pump-probe studies, the thesis presents the absorption spectra of mono- and bilayer graphene including the impact of the fully momentum-dependent optical and Coulomb matrix elements. In agreement with recent experiments, the absorbance of graphene is characterized by a frequency-independent value in the near-infrared spectral region and a pronounced peak in the ultraviolet region resulting from interband transition. In contrast to the linear band structure of graphene, the energy dispersion of bilayer graphene exhibits four parabolic bands resulting in interesting optical features: The absorbance exhibits in the low energy spectrum a pronounced peak, which can be unambiguously ascribed to interband cross transitions at the Dirac point. In the ultraviolet region, the spectrum is characterized by two pronounced energetically close absorption peaks. For both mono- and bilayer graphene, the calculations reveal the importance of the Coulomb interaction resulting in the formation of saddle-point excitons.

Finally, the relaxation dynamics in doped graphene is discussed, where the main focus lies on the carrier multiplication. This is an interesting ultrafast phenomenon that is related to the linear electronic band structure of graphene opening up the possibility of efficient Coulomb-induced Auger processes. Introducing doping, the Coulomb-induced processes of carrier multiplication $(\mathrm{CM})$ and hot carrier multiplication (hCM) are to be distinguished: Auger scattering bridging the valence and the conduction band gives rise to CM corresponding to an increase of the number of charge carriers in graphene. In contrast, hCM is induced by Coulomb-induced intraband scattering bridging the states below and above the Fermi level and resulting in an increase of the number of hot carriers. Comparing the theoretical results with recent time-resolved ARPES measurements, an excellent agreement on the temporal evolution of the hot carrier multiplication for $\mathrm{n}$ - and $\mathrm{p}$-doped graphene is found. 



\section{Contents}

1. Introduction 1

1.1. Motivation . . . . . . . . . . . . . . 1

1.2. Structure of the thesis . . . . . . . . . . . . . 3

2. Theoretical model 4

2.1. Hamiltonoperator . . . . . . . . . . . . . . . 4 4

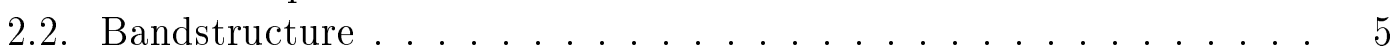

2.2.1. Unit cell and Brillouin zone of graphene and bilayer graphene 5

2.2.2. $\quad$ Bandstructure of graphene . . . . . . . . . 7

2.2.3. Bandstructure of bilayer graphene . . . . . . . . . . . 10

2.3. Matrix elements . . . . . . . . . . . . . . . . 12

2.3.1. Optical matrix element . . . . . . . . . . . . . 12

2.3.2. Coulomb matrix element . . . . . . . . . . . . . . . 16

2.3.3. Electron-phonon matrix elements . . . . . . . . . . . . . 19

2.4. Microscopic Bloch equation . . . . . . . . . . . . 21

2.4.1. Hartree-Fock approximation . . . . . . . . . . . . . . 23

2.4.2. Second-order Born-Markov approximation . . . . . . . . . 23

2.5. Differential transmission spectroscopy . . . . . . . . . . . 27

\begin{tabular}{ll}
3. & Absorption spectra \\
\hline
\end{tabular}

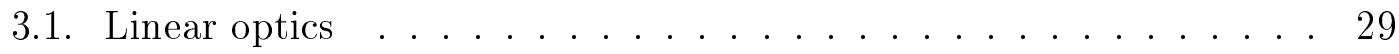

3.2. Excitonic absorption spectra . . . . . . . . . . . . . . . . . . . . . . . . . . . 31

3.2.1. Graphene ..................... . . 31

3.2.2. $\quad$ Bilayer graphene . . . . . . . . . . . . . . . 34

\begin{tabular}{|l|l|}
\hline 4. Impact of intraband absorption in graphene & 37
\end{tabular}

4.1. Phonon-assisted intraband absorption processes . . . . . . . . . . 38

4.2. Transient negative differential transmission . . . . . . . . . . . . . 41

$\begin{array}{ll}\text { 5. Terahertz carrier relaxation dynamics in graphene } & 47\end{array}$

5.1. Terahertz carrier dynamics . . . . . . . . . . . . . 48

5.1.1. Hot-carrier dynamics in graphene . . . . . . . . . . . . . . 48

5.1.2. $\quad$ Perturbation approach for the $\mathrm{THz}$ dynamics . . . . . . . 50 
5.2. Optical pump and $\mathrm{THz}$ probe differential transmission spectra . . . 51

5.2.1. Highly doped graphene . . . . . . . . . . . . . 52

5.2 .2$. Undoped graphene . . . . . . . . . . . . . 56

5.3. Shortcoming of the Drude model . . . . . . . . . . . . 60

6. Impact of doping on carrier relaxation dynamics in graphene 66

6.1 Carrier occupation and polarization . . . . . . . . . . . 67

6.2. Orientational carrier relaxation . . . . . . . . . . . . . 69

6.3. Carrier Multiplication in doped graphene . . . . . . . . . . . . . 72

6.3.1. Statictical approach for the purely Coulomb-induced carrier

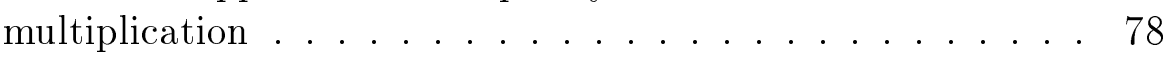

\begin{tabular}{ll}
\hline 7. Conclusion & 83
\end{tabular}

\begin{tabular}{|l|l}
\hline A. Optical matrix elements & I
\end{tabular}

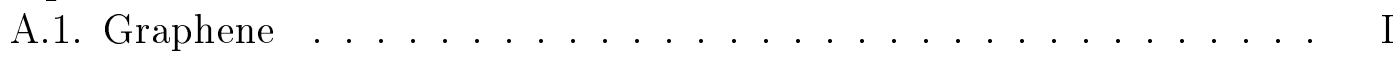

A.1.1. Linearised matrix elements in graphene . . . . . . . . . . . II

A.2. Bilayer graphene ...................... III

B. Coulomb matrix element IV

C. Derivation of the Coulomb- and phonon-induced dynamics VI

C.1. Coulomb interaction .................. VI

C.2. Electron-phonon coupling . . . . . . . . . . . . IX

C.2.1. Phonon dynamics . . . . . . . . . . . . XII

D. Derivation of the dephasing in the dynamical screening XIII

E. Perturbation approach of the phonon-assisted quantities XVI

\begin{tabular}{ll}
\hline F. Terahertz dynamics & XXI
\end{tabular}

F.1. Electron-Phonon Scattering . . . . . . . . . . . . . . . XXI

F.2. Electron-Electron Scattering . . . . . . . . . . . . . . XXII

F.3. Drude-like intraband current . . . . . . . . . . . . . . . XXXIII

G. Carrier and energy density $\quad$ XXIV 


\section{Introduction}

\subsection{Motivation}

During the past few decades, nanomaterials, such as thin films and carbon structures, entered the forefront of research in materials science. These carbon materials includes fullerones, nanotubes, nanoribbons, and recently prepared single layer of graphite: graphene [1], cp. Fig. 1.1. For a long time it was presumed that freestanding graphene can never be found in nature, since strictly two-dimensional crystals could not exist due to the thermodynamically instability |2,3|. However, in 2004, Novoselov et al. demonstrates that graphene can be isolated from graphite by a machanical exfoliation technique on a surface [1]. The existence was later confirmed by an unusual qunatum Hall effect 4,5. Since then, graphene has been a subject of research due its remarkable electronic, mechanical, optical, and chemical properties and potential for future applications [6]. Graphene as a monolayer of carbon atoms in the form of a strict two-dimensional hexagonal lattice structure provides a lot fascinating physics. It has a unique bandstructure, which is fundamentally different from anything that was known before. The linear dispersion in the low-energy regime giving rise to many extraordinary properties, where the electrons behave like relativistic massless particles. Hence, the electrons are called Dirac fermions and can be described by a Dirac-like equation. Although the density of states vanishes at the Dirac points, graphene exhibits a conductivity of order $2 e^{2} / \hbar$ at these points. Graphene is one of the strongest materials, which can be traced back to the strong $\mathrm{sp}^{2}$-bonds between the carbon atoms. Furthermore, it conducts heat and electricty with a large efficiency due to the large carrier mobility. Recent investigations consider graphene as a saturable absorber in laser systems, as a transparent electrode in solar cells or as ultrafast photodetectors. Because of these unique electronic properties, graphene is one of the most promising materials for several applications in optoelectronics and nanoelectronics. Many claim that graphene has the potential to replace silicon in electronic devices. Another interesting material, which will be part of this thesis is Bilayer graphene. Bilayer graphene consisting of weakly coupled two carbon monolayers exhibits also remarkable optical and electronic properties [7-10]. Most significantly, the interlayer coupling changes the energy dispersion from linear to parabolic 17,9,11,12]. The possibilty to break the layer symmetry of bilayer graphene by applying an 


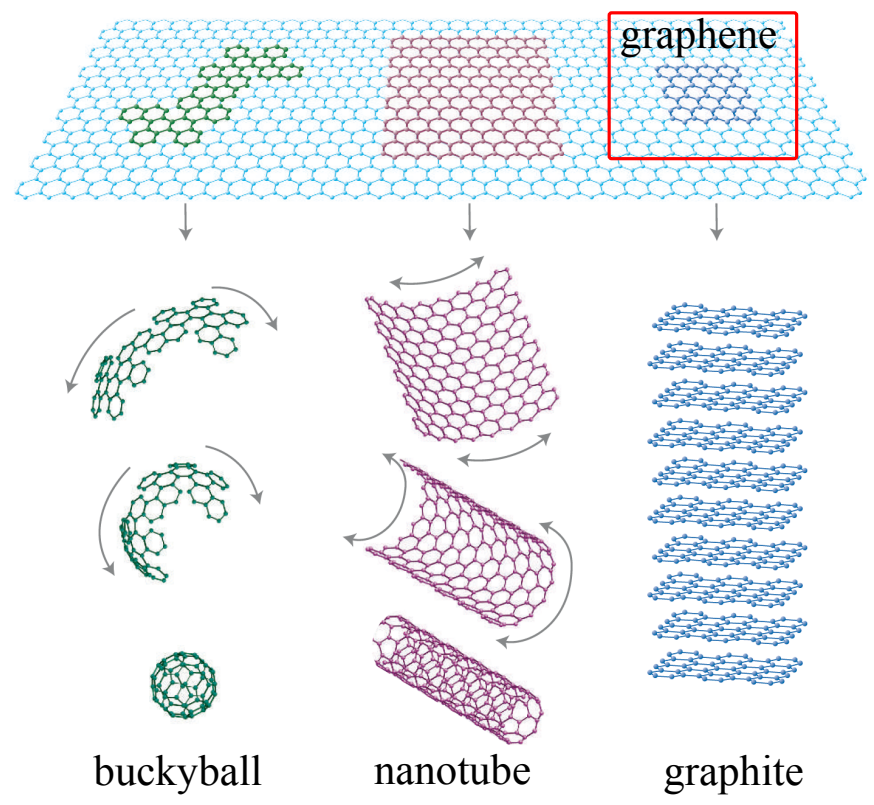

Figure 1.1.: Graphene can be seen as the two-dimensional building material for other forms as buckyballs (0D), nanotubes (1D), and graphite (3D). Figure is taken from $|6|$.

external field is promising for the design of future nanoelectronic devices [11-16]. For all these applications it is of crucial importance to understand the dynamics of excited carriers in carbon structures.

In the last few years a number of theoretical and experimental studies were performed to obtain a deep understanding of the carrier relaxation dynamics in graphene [17-27]. In graphene, a linearly polarized ultrafast optical pulse excitation gives rise to an initially anisotropic distribution of carriers at high energy [28, 29]. The efficient carrier-carrier and carrier-phonon scattering processes quickly relax the hot carriers into an isotropic thermal distribution, which is then followed by carrier cooling due to carrier-phonon processes [17, 22, 23]. A variety of dynamical phenomena, such as the appearance of a significant carrier multiplication and transient optical gain, have been theoretically predicted $[30-33]$ and experimentally confirmed $[32,34,35]$. A consistent treatment of the relaxation dynamics containing all important scattering contributions can be realized within the density matrix approach $17,36,37$. 


\section{The main questions addressed in this work are:}

1. What characteristic features exhibit the absorption spectrum of mono- and bilayer graphene and how important are Coulomb-induced effects?

2. Can phonon-assisted intraband absorption processes explain the transient negative differential transmission in graphene, which has been observed in many optical pump-probe experiments?

3. How does the relaxation dynamics behave in the terahertz regime for undoped and highly doped graphene, which have been interpreted in the framework of phenomenological models so far, but have not been understood in terms of fundamental microscopic theory?

4. Finally, how does the relaxation dynamics change by introducing doping with respect to the orientational dynamics and carrier multiplication?

The thesis will follow the treatment of these questions within the density matrix approximation. Furthermore, the obtained results will be compared with recent experiments.

\subsection{Structure of the thesis}

In chapter 2 the thereotical background of the many-particle density matrix theory is introduced. Here, the underlying Hamilton operator is provided including a detailed description of the specific band structure, matrix elements. Furthermore, the Bloch equations are derived, which is the key to obtain the microscopic picture of the nonequilibrium dynamics in graphene. The exitonic absorption spectrum of mono- and bilayer graphene including a detailed microscopic study of all allowed optical transitions is presented in chapter 3. Chapter 4 discusses the influence of phonon-assisted intraband on the differential transmission spectra, where a microscopic explanation for the occurrence of transient negative differential transmission in graphene is given. The next chapter gives insight in the terahertz dynamics of graphene, where optical pump and terahertz probe differential transmission spectra are discussed. Finally, chapter 5 predicts the occurrence of a significant hot carrier multiplication in doped graphene. 


\section{Theoretical model}

In this chapter a summary of the theoretical basics is introduced to describe the linear absorption as well as the relaxation dynamics of nonequilibrium carrier in graphene. The microscopic approach is based on the density matrix formalism, where the quantum mechanical Heisenberg picture is applied and electronic quantities and phonon interaction are described in second quantization. First, in section 2.1. the complete Hamilton operator in second quantization is presented. In the sections 2.2 and 2.3, the graphene and bilayer graphene bandstructure, the specific optical and Coulomb matrix elements are derived within the tight-binding approach. Furthermore, the electron-phonon matrix element for different modes is shown. In section 2.4 the derivation of the optical graphene Bloch equations, which describe the coupled dynamics of carriers, phonons and the microscopic polarization, is presented. Finally the chapter concludes with the theoretical modelling of the differential transmission spectrum, which is required to compare the numerical results with the experiment.

\subsection{Hamiltonoperator}

The starting point of the theoretical approach is the many-particle Hamiltonoperator

$$
H=H_{0}+H_{c, l}+H_{c, p}+H_{c, c}+H_{c, p},
$$

which is expressed within the formalism of second quantization. The contributions in Eq. 2.1 can be expressed by the creation and annihilation operators, where $a_{l}^{+}$ $\left(a_{l}\right)$ creates (annihilates) an electron in the quantum state $l=(\lambda, \mathbf{k})$. The compound index $l$ contains the wave vector $\mathbf{k}$ and the band index $\lambda$. The phonon operators $b_{u}^{\dagger}$ and $b_{u}$ act on the phonon states $u=\left(\mathbf{q}_{u}, j_{u}\right)$ with the phonon momentum $\mathbf{q}$ and the phonon mode $j$. The non-interaction part $H_{0}$ is determined by the electric single-particle energy $\varepsilon_{l}$ and phonon dispersion $\omega_{u}$ and reads

$$
H_{0}=\sum_{l} \varepsilon_{l} a_{l}^{\dagger} a_{l}+\sum_{u} \hbar \omega_{u}\left(b_{u}^{\dagger} b_{u}+\frac{1}{2}\right) .
$$

The efficiency of carrier-light coupling is determined by the contribution $H_{c, l}$ in the complete Hamilton operator, which reads within the radiation gauge and the 
dipole approximation 38

$$
H_{c, l}=i \hbar \frac{e_{0}}{m_{0}} \sum_{l_{1}, l_{2}} \mathbf{M}_{l_{1}, l_{2}} \cdot \mathbf{A}(t) a_{l_{1}}^{+} a_{l_{2}}
$$

with the elementary electron charge $e_{0}$, the free electron mass $m_{0}$, and the vector potential $\mathbf{A}(t)$. The optical matrix element $\mathbf{M}_{\mathbf{k} \mathbf{k}^{\prime}}^{\lambda \lambda^{\prime}}$ determines the coupling strength between the electronic system and an external field. A detailed discussion of the optical matrix element is provided in section 2.3 .

The carrier-carrier interaction $H_{c, c}$ in the Hamilton operator is given by

$$
H_{c, c}=\frac{1}{2} \sum_{l_{1}, l_{2}, l_{3}, l_{4}} V_{l_{3} l_{4}}^{l_{1} l_{2}} a_{l_{1}}^{\dagger} a_{l_{2}}^{\dagger} a_{l} a_{l_{3}}
$$

with $V_{l_{3} l_{4}}^{l_{1} l_{2}}=\int \mathrm{d} \mathbf{r} \int \mathrm{d} \mathbf{r}^{\prime} \Psi_{l_{1}}^{*}(\mathbf{r}) \Psi_{l_{2}}^{*}\left(\mathbf{r}^{\prime}\right) V_{\text {Coul }}\left(\mathbf{r}-\mathbf{r}^{\prime}\right) \Psi_{l_{4}}(\mathbf{r}) \Psi_{l_{3}}\left(\mathbf{r}^{\prime}\right)$ as the Coulomb matrix element, which will be discussed in section 2.3.2 in detail. The last Hamilton operator $H_{c, p}$ describes the interaction between electrons and phonons and reads in second quantization

$$
H_{c, p}=\sum_{\lambda \lambda^{\prime} \mathbf{k}} \sum_{j \mathbf{q}}\left[g_{\mathbf{k}, \mathbf{q}}^{\lambda \lambda^{\prime} j} a_{\mathbf{k}}^{\lambda \dagger} a_{\mathbf{k}-\mathbf{q}}^{\lambda^{\prime}} b_{\mathbf{q}}^{j}+g_{\mathbf{k}, \mathbf{q}}^{\lambda \lambda^{\prime} j *} a_{\mathbf{k}-\mathbf{q}}^{\lambda^{\prime} \dagger} a_{\mathbf{k}}^{\lambda} b_{\mathbf{q}}^{j \dagger}\right] .
$$

The strength of the carrier-phonon interaction is determined by the corresponding electron-phonon coupling elements $g_{\mathbf{k}, \mathbf{q}}^{\lambda \lambda^{\prime} j}$ considering the optical as well as the acoustic modes [39, 40]. The first term of $H_{c, p}$ can be interpreted as an electronic transition from the state $\mathbf{k}-\mathbf{q}$ to the state $\mathbf{k}$ induced by an annihilation of a photon with the momentum $\mathbf{q}$, whereas the second term describes the inverse processes initiated by the creation of a phonon. A detailed description of the electron-phonon matrix element is presented in section 2.3.3.

The Hamilton operator becomes specific by determine the bandstructure and matrix elements. In the next two sections 2.2 and 2.3 , tight-binding wave functions are applied to analytically work out the bandstructure, the optical as well as the Coulomb matrix elements of graphene and bilayer graphene.

\subsection{Bandstructure}

\subsubsection{Unit cell and Brillouin zone of graphene and bilayer graphene}

Graphene is assumed to be a perfectly two-dimensional crystal consisting of carbon atoms arranged in a hexagonal lattice, cf. Fig. 2.1(a). The unit cell contains two 
(a)

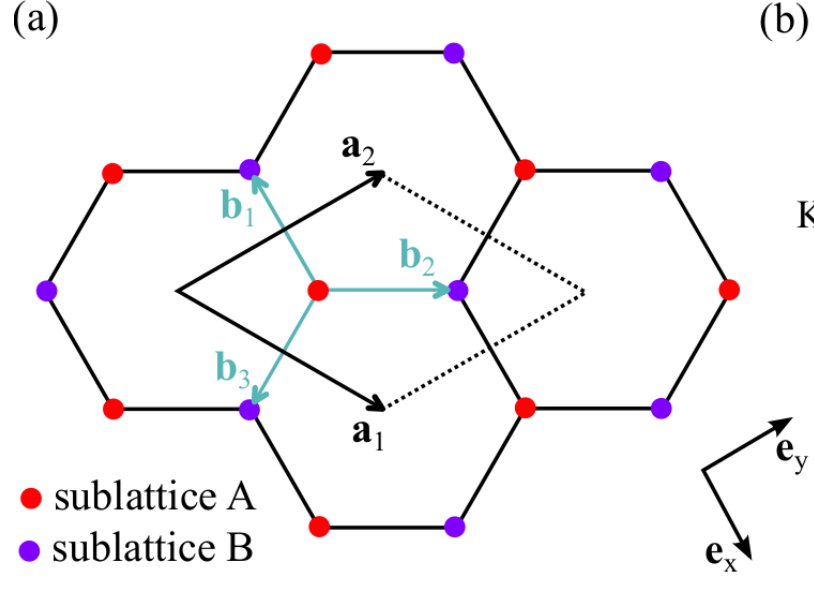

(b)

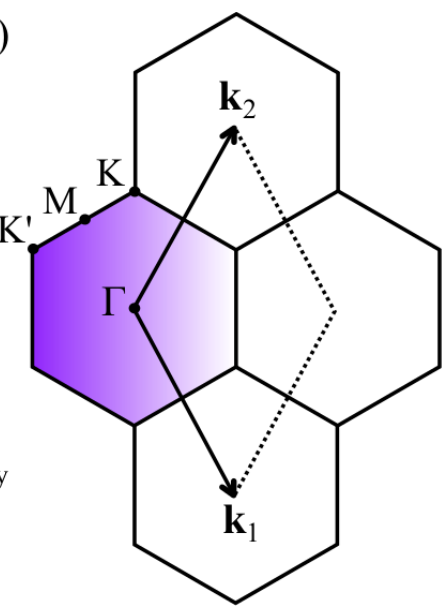

Figure 2.1.: (a) Direct lattice of graphene with the two subatoms A and B. The unit cell is spanned by the two lattice vectors $\mathbf{a}_{1}$ and $\mathbf{a}_{2}$. (b) Brillouin zone (blue area) of graphene with the reciprocal lattice vectors $\mathbf{k}_{1}$ and $\mathbf{k}_{2}$ and the high symmetry points $\Gamma, M, K$ and $K^{\prime}$.

atoms $\mathrm{A}$ and $\mathrm{B}$ and is spanned by the two lattice vectors $\mathbf{a}_{1}$ and $\mathbf{a}_{2}$ :

$$
\mathbf{a}_{1}=a_{0}\left(\begin{array}{c}
\frac{\sqrt{3}}{2} \\
\frac{1}{2}
\end{array}\right) \text { and } \mathbf{a}_{2}=a_{0}\left(\begin{array}{l}
0 \\
1
\end{array}\right)
$$

where the length $a_{0}=0,246 \mathrm{~nm}$ is the lattice constant [41]. The hopping vectors $\mathbf{b}_{i}$ from a carbon atom in the sublattice $A$ to its three neighbour atoms in sublattice $B$ are given by

$$
\mathbf{b}_{1}=a_{0}\left(\begin{array}{c}
-\frac{1}{\sqrt{3}} \\
0
\end{array}\right), \mathbf{b}_{2}=a_{0}\left(\begin{array}{c}
\frac{1}{2 \sqrt{3}} \\
-\frac{1}{2}
\end{array}\right) \text { and } \mathbf{b}_{3}=a_{0}\left(\begin{array}{c}
\frac{1}{2 \sqrt{3}} \\
\frac{1}{2}
\end{array}\right) .
$$

The reciprocal lattice including the first Brillouin zone is shown in Fig. 2.1(b). It contains the high-symmetry $\Gamma, K, K^{\prime}$ and $M$ points and is spanned by the reciprocal vectors $\mathbf{k}_{1}$ and $\mathbf{k}_{2}$ :

$$
\mathbf{k}_{1}=a_{0}^{-1}\left(\begin{array}{c}
\frac{4 \pi}{\sqrt{3}} \\
0
\end{array}\right) \text { and } \mathbf{k}_{2}=a_{0}^{-1}\left(\begin{array}{c}
-\frac{2 \pi}{\sqrt{3}} \\
2 \pi
\end{array}\right) .
$$

In layered carbon sheets, there are several ways of stacking. As graphite, bilayer graphene typically exists in Bernal-type stacking [10, 42], where the $A_{1}$ atoms in the first monolayer are directly stacked over the $B_{2}$ atoms of the second layer, cp. Fig. 2.2. The unit cell of bilayer graphene contains the atoms $A_{1}, A_{2}, B_{1}$ and $B_{2}$. The interaction parameters $\gamma_{1}, \gamma_{3}$ and $\gamma_{4}$ will be explicitly determined in the next section. In this thesis, only the Bernal-type stacking is considered. 


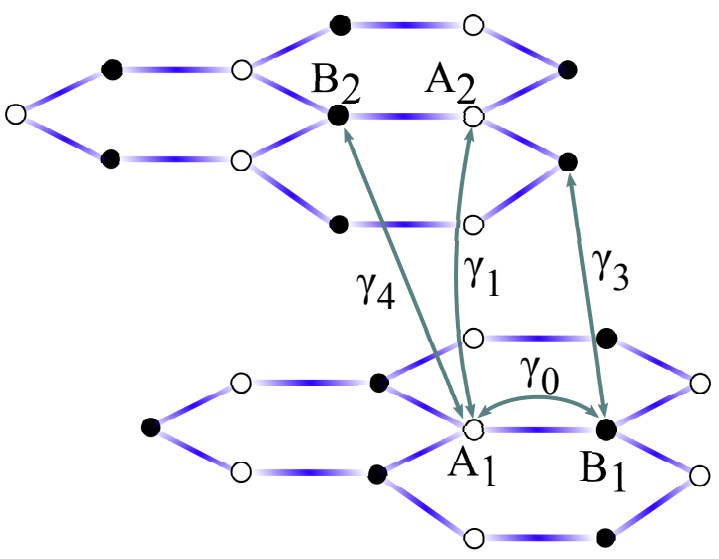

Figure 2.2.: The geometric structure of bilayer graphene with Bernal stacking. Here, $\gamma_{1}$ represents the interaction between A and B atoms from two neighbouring carbon layers, while $\gamma_{0}$ denotes the coupling between the two next atoms within one layer. Furthermore, $\gamma_{3}$ describes the coupling between the subatoms $B_{1}$ and $B_{2}$ and $\gamma_{4}$ between $A_{1}$ and $B_{2}$, respectively.

\subsubsection{Bandstructure of graphene}

The bandstructure of graphene is formed by four valence electrons, two 2s-electrons and two 2p-electrons. Respectively, three two 2 s-electrons and one 2 p-electron configure an $\mathrm{sp}^{2}$ - hybridization, being responsible for the strong in-plane binding with the carbon-carbon distance of $0.142 \mathrm{~nm}$ in graphene [41]. The remaining valence electron forms a $\pi$-bond with the neighbouring carbon atoms. By using a tight-binding approach for the $\pi$-electrons from the $2 p_{z}$-orbitals, the low-energy electronic properties of mono- as well as bilayer graphene turn out to be captured in good approximation. The starting point to determine the bandstructure is the Schroedinger equation:

$$
H \Psi_{\mathbf{k}}^{\lambda}(\mathbf{r})=\varepsilon_{\mathbf{k}}^{\lambda} \Psi_{\mathbf{k}}^{\lambda}(\mathbf{r})
$$

The wave function $\Psi_{\mathbf{k}}^{\lambda}(\mathbf{r})$ can be approximated within the tight-binding approach as a linear combination of both equivalent sublattices $\Phi_{A}$ and $\Phi_{B}$

$$
\Psi_{\mathbf{k}}^{\lambda}(\mathbf{r})=C_{A}^{\lambda}(\mathbf{k}) \Phi_{A}(\mathbf{k}, \mathbf{r})+C_{B}^{\lambda}(\mathbf{k}) \Phi_{B}(\mathbf{k}, \mathbf{r}),
$$

with the tight-binding coefficients $C_{s}^{\lambda}(\mathbf{k})$. The sublattice wave functions are described by Bloch functions:

$$
\Phi_{l}(\mathbf{k}, \mathbf{r})=\frac{1}{\sqrt{N}} \sum_{\mathbf{R}_{s}} e^{i \mathbf{k} \mathbf{R}_{s}} \phi\left(\mathbf{r}-\mathbf{R}_{s}\right),
$$


where $N$ is the number of unit cells in the system. $\mathbf{R}_{s}$ are the corresponding lattice vector and $\phi\left(\mathbf{r}-\mathbf{R}_{l}\right)$ the $2 p_{z}$-orbital function, which are approximated by $2 p_{z}$-orbitals of the hydrogen atom with an effective atomic number $Z_{\text {eff }}$. Inserting this expression into Eq. 2.10 and multiplying with $\Phi_{A}^{*}(\mathbf{k}, \mathbf{r})$ and $\Phi_{B}^{*}(\mathbf{k}, \mathbf{r})$, the integration yields

$$
\left(\begin{array}{ll}
H_{A A}-\varepsilon_{\mathbf{k}} S_{A A} & H_{A B}-\varepsilon_{\mathbf{k}} S_{A B} \\
H_{B A}-\varepsilon_{\mathbf{k}} S_{B A} & H_{B B}-\varepsilon_{\mathbf{k}} S_{B B}
\end{array}\right) \cdot\left(\begin{array}{l}
C_{A}(\mathbf{k}) \\
C_{B}(\mathbf{k})
\end{array}\right)=0 .
$$

with $S_{i j}=\left\langle\phi_{i} \mid \phi_{j}\right\rangle$ and $H_{i j}=\left\langle\phi_{i}|H| \phi_{j}\right\rangle$. Since the sublattices $A$ and $B$ are equal, the elements fulfil following symmetries:

$$
\begin{gathered}
H_{A A}=H_{B B}, H_{A B}=H_{B A}^{*}, \\
S_{A A}=S_{B B} \text { and } S_{A B}=S_{B A}^{*} .
\end{gathered}
$$

Equation 2.12 exhibits non-trivial solutions, if the eigenvalues fulfil

$$
\varepsilon_{\mathbf{k}}^{1,2}=\frac{H_{A A} S_{A A}-\operatorname{Re}\left[H_{A B} S_{A B}^{*}\right]}{S_{A A}^{2}-\left|S_{A B}\right|^{2}} \pm \sqrt{\left(\frac{\operatorname{Re}\left[H_{A B} S_{A B}^{*}\right]-H_{A A} S_{A A}}{S_{A A}^{2}-\left|S_{A B}\right|^{2}}\right)^{2}-H_{A A}^{2}+\left|H_{A B}^{2}\right|} .
$$

To obtain a specific solution the matrix elements $H_{i j}$ and $S_{i j}$ have to be calculated. The element $H_{A A}(\mathbf{k})$ reads within the tight-binding approximation

$$
H_{A A}(\mathbf{k})=\left\langle\phi_{A}|H| \phi_{A^{\prime}}\right\rangle=\frac{1}{N} \sum_{\mathbf{R}_{A}} \sum_{\mathbf{R}_{A^{\prime}}}\left\langle e^{i \mathbf{k} \mathbf{R}_{A}} \phi\left(\mathbf{r}-\mathbf{R}_{A}\right)|H| e^{i \mathbf{k} \mathbf{R}_{A^{\prime}}} \phi\left(\mathbf{r}-\mathbf{R}_{A^{\prime}}\right)\right\rangle .
$$

Due to the localization of the atomic orbital, $H_{i j}$ can be treated within the nearest neighbour approximation [41]. That means for each summand $\mathbf{R}_{A}$ only the three adjacent $B$ atoms at $R_{B_{i}}=R_{A}+b_{i}$ contribute, where the $b_{i}$ with $i=1,2,3$ are the nearest neighbour vectors, which are shown in Fig. 2.1. Note that the opposite case, i.e. fixing the $B$-sublattice and considering the nearest $A$-atoms, leads to the same eigenvalues.

$$
H_{A A}(\mathbf{k})=\frac{1}{N} \sum_{\mathbf{R}_{A}} e^{i \mathbf{k} \mathbf{R}_{A}} e^{-i \mathbf{k} \mathbf{R}_{A}}\left\langle\phi\left(\mathbf{r}-\mathbf{R}_{A}\right)|H| \phi\left(\mathbf{r}-\mathbf{R}_{A}\right)\right\rangle=\frac{N}{N} \varepsilon_{0}=\varepsilon_{0}
$$

with $\sum_{\mathbf{R}_{A}}=N$ and $\varepsilon_{0}=\left\langle\phi\left(\mathbf{r}-\mathbf{R}_{A}\right)|H| \phi\left(\mathbf{r}-\mathbf{R}_{A}\right)\right\rangle$. The non-diagonal matrix element $H_{A B}$ reads

$$
H_{A B}(\mathbf{k})=\left\langle\phi_{A}|H| \phi_{B}\right\rangle=\frac{1}{N} \sum_{\mathbf{R}_{A}} \sum_{\mathbf{R}_{B}}\left\langle e^{i \mathbf{k} \mathbf{R}_{A}} \phi\left(\mathbf{r}-\mathbf{R}_{A}\right)|H| e^{i \mathbf{k} \mathbf{R}_{B}} \phi\left(\mathbf{r}-\mathbf{R}_{B}\right)\right\rangle .
$$


(a)

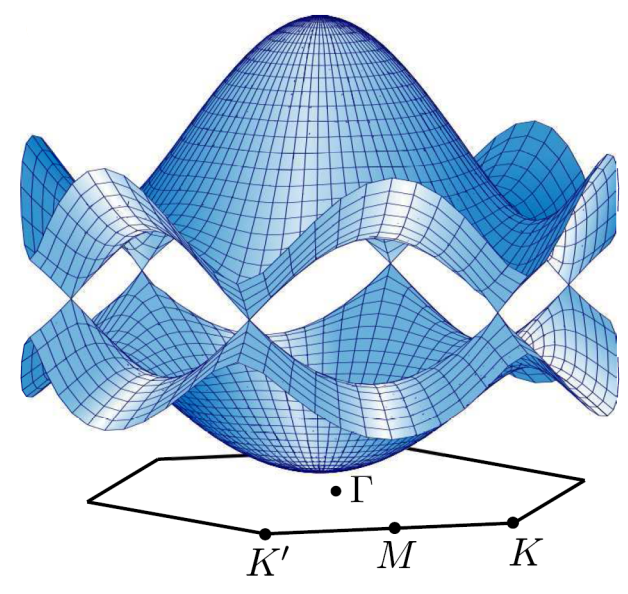

(b)

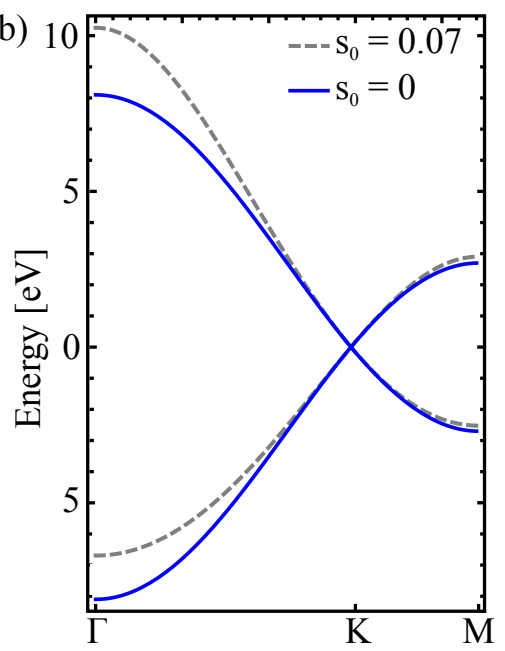

Figure 2.3.: (a) Bandstructure of graphene illustrating the valence and the conduction band along the Brillouin zone. (b) The bandstructure along the highsymmetry $\Gamma K M$ line showing the influence of the interaction parameter $s_{0}$. Subfigure (a) is adopted from [17.

With $\mathbf{R}_{B}-\mathbf{R}_{A}=\mathbf{b}_{i}$ the matrix element $H_{A B}$ results in

$$
H_{A B}(\mathbf{k})=\frac{1}{N} \sum_{\mathbf{R}_{A}} \sum_{i=1}^{3} e^{i \mathbf{k} \mathbf{b}_{i}}\left\langle\phi\left(\mathbf{r}-\mathbf{R}_{A}\right)|H| \phi\left(\mathbf{r}-\mathbf{R}_{A}-\mathbf{b}_{i}\right)\right\rangle=\gamma_{0} e(\mathbf{k}) .
$$

with the carbon-carbon interaction parameter $\gamma_{0}=\left\langle\phi\left(\mathbf{r}-\mathbf{R}_{A}\right)|H| \phi\left(\mathbf{r}-\mathbf{R}_{A}-\mathbf{b}_{i}\right)\right\rangle$. The function $e(\mathbf{k})$, which describes the contribution from the three nearest neighbour atoms, reads

$$
e(\mathbf{k})=\sum_{i=1}^{3} e^{i \mathbf{k b}_{i}}
$$

The matrix elements $S_{A A}$ and $S_{A B}$ are derived analogously:

$$
\begin{aligned}
& S_{A A}=1 \\
& S_{A B}=\left\langle\phi\left(\mathbf{r}-\mathbf{R}_{A}\right) \mid \phi\left(\mathbf{r}-\mathbf{R}_{A}-\mathbf{b}_{i}\right)\right\rangle e(\mathbf{k})=s_{0} e(\mathbf{k})
\end{aligned}
$$

with the overlap integral between two neighbouring atoms $s_{0}$. Inserting the four derived matrix element into Eq. 2.14 leads to the bandstructure of graphene

$$
\varepsilon_{\mathbf{k}}^{\lambda}=\frac{\varepsilon_{0}-\sigma_{\lambda} \gamma_{0}|e(\mathbf{k})|}{\sigma_{\lambda} s_{0}|e(\mathbf{k})|}
$$


where $\lambda=(c, v)$ denotes the conduction $\left(\sigma_{c}=1\right)$ and valance $\left(\sigma_{v}=-1\right)$ band. The three parameter $\varepsilon_{0}, \gamma_{0}$, and $s_{0}$ are determined by fitting to experimental data. In this thesis, $\varepsilon_{0}=0 \mathrm{eV}, \gamma_{0}=-2.7 \mathrm{eV}$ and $s_{0}=0.07$ are used $[43$. The absolute value of the function $e(\mathbf{k})$ can be written as

$$
|e(\mathbf{k})|=\sqrt{3+4 \cos \left(\frac{\sqrt{3} a_{0}}{2} k_{x}\right) \cos \left(\frac{a_{0}}{2} k_{y}\right)+2 \cos \left(a_{0} k_{y}\right)} .
$$

Figure 2.3(a) illustrates the bandstructure of graphene offering a saddle at the $M$ point and a maximal band gap at the $\Gamma$ point. The overlap integral $s_{0}$ [cp. Fig. 2.3(b)] breaks the electron-hole symmetry (for undoped graphene), but since its influence in the optically relevant region $E<4 \mathrm{eV}$ is vanishingly small it is neglected in the following. The conduction and valence band cross at the $K$ and $K^{\prime}$ point resulting in a vanishing bandgap. The region around these Dirac points can be approximated by a linear electron dispersion

$$
\varepsilon_{\mathbf{k}}^{\lambda}=\sigma_{\lambda} \hbar v_{F}|\mathbf{k}|
$$

with the Fermi velocity $v_{F}=\gamma_{0} \sqrt{3} a_{0} / 2=0.605 \mathrm{eVnm}$.

\subsubsection{Bandstructure of bilayer graphene}

In bilayer graphene, the wave function $\Psi_{\mathbf{k}}^{\lambda}(\mathbf{r})$ can be approximated within the tight-binding approach as a linear combination of the equivalent sublattices $\phi_{A_{1,2}}$ and $\phi_{B_{1,2}}[44]$ :

$$
\Psi_{\mathbf{k}}^{\lambda}(\mathbf{r})=\sum_{\substack{s=A_{1}, A_{2} \\ B_{1}, B_{2}}} C_{s}^{\lambda}(\mathbf{k}) \frac{1}{\sqrt{N}} \sum_{\mathbf{R}_{s}} e^{i \mathbf{k} \mathbf{R}_{s}} \phi\left(\mathbf{r}-\mathbf{R}_{s}\right) .
$$

In analogy to graphene, the matrix equation for bilayer graphene is given by

$$
\left(\begin{array}{cccc}
H_{A_{1} A_{1}}-\varepsilon_{\mathbf{k}} S_{A_{1} A_{1}} & H_{A_{1} B_{1}}-\varepsilon_{\mathbf{k}} S_{A_{1} B_{1}} & H_{A_{1} A_{2}}-\varepsilon_{\mathbf{k}} S_{A_{1} A_{2}} & H_{A_{1} B_{2}}-\varepsilon_{\mathbf{k}} S_{A_{1} B_{2}} \\
H_{B_{1} A_{1}}-\varepsilon_{\mathbf{k}} S_{B_{1} A_{1}} & H_{B_{1} B_{1}}-\varepsilon_{\mathbf{k}} S_{B_{1} B_{1}} & H_{B_{1} A_{2}}-\varepsilon_{\mathbf{k}} S_{B_{1} A_{2}} & H_{B_{1} B_{2}}-\varepsilon_{\mathbf{k}} S_{B_{1} B_{2}} \\
H_{A_{2} A_{1}}-\varepsilon_{\mathbf{k}} S_{A_{2} A_{1}} & H_{A_{2} B_{1}}-\varepsilon_{\mathbf{k}} S_{A_{2} B_{1}} & H_{A_{2} A_{2}}-\varepsilon_{\mathbf{k}} S_{A_{2} A_{2}} & H_{A_{1} B_{2}}-\varepsilon_{\mathbf{k}} S_{A_{2} B_{2}} \\
H_{B_{2} A_{1}}-\varepsilon_{\mathbf{k}} S_{B_{2} A_{1}} & H_{B_{2} B_{1}}-\varepsilon_{\mathbf{k}} S_{B_{2} B_{1}} & H_{B_{2} A_{2}}-\varepsilon_{\mathbf{k}} S_{B_{2} A_{2}} & H_{B_{2} B_{2}}-\varepsilon_{\mathbf{k}} S_{B_{2} B_{2}}
\end{array}\right)\left(\begin{array}{l}
C_{A_{1}} \\
C_{B_{1}} \\
C_{A_{2}} \\
C_{B_{2}}
\end{array}\right)=0 .
$$

These matrix elements fulfil the following symmetries (the atoms of the sublattice $A_{1,2}$ and $B_{1,2}$ are equal):

$$
\begin{aligned}
& H_{A_{1} A_{1}}=H_{B_{1} B_{1}}=H_{A_{2} A_{2}}=H_{B_{2} B_{2}}, \\
& H_{A_{1} B_{1}}=H_{B_{2} A_{2}}=H_{B_{1} A_{1}}^{*}=H_{A_{2} B_{2}}^{*}, \\
& H_{A_{1} A_{2}}=H_{A_{1} A_{2}}, \quad H_{B_{1} B_{2}}=H_{B_{2} B_{1}}^{*}, \\
& H_{B_{2} A_{1}}=H_{A_{2} B_{1}}=H_{B_{1} A_{2}}^{*}=H_{A_{1} B_{2}}^{*} .
\end{aligned}
$$


The elements $S_{i j}$ have the same symmetries. The matrix element $H_{A_{1} A_{1}}(\mathbf{k})$ reads within the tight-binding approximation

$$
\begin{aligned}
H_{A_{1} A_{1}}(\mathbf{k}) & =\left\langle\phi_{A_{1}}|H| \phi_{A_{1}^{\prime}}\right\rangle=\frac{1}{N} \sum_{\mathbf{R}_{A_{1}}} \sum_{\mathbf{R}_{A_{1}^{\prime}}}\left\langle e^{i \mathbf{k} \mathbf{R}_{A_{1}}} \phi\left(\mathbf{r}-\mathbf{R}_{A_{1}}\right)|H| e^{\left.i \mathbf{k} \mathbf{R}_{A_{1}^{\prime}} \phi\left(\mathbf{r}-\mathbf{R}_{A_{1}^{\prime}}\right)\right\rangle}\right. \\
& =\frac{1}{N} \sum_{\mathbf{R}_{A_{1}}} e^{i \mathbf{k} \mathbf{R}_{A_{1}}} e^{-i \mathbf{k} \mathbf{R}_{A}}\left\langle\phi\left(\mathbf{r}-\mathbf{R}_{A_{1}}\right)|H| \phi\left(\mathbf{r}-\mathbf{R}_{A_{1}}\right)\right\rangle=\frac{N}{N} \varepsilon_{0}=\varepsilon_{0} .
\end{aligned}
$$

The element $H_{A_{1} B_{1}}(\mathbf{k})$ corresponds with the matrix element of graphene $H_{A B}(\mathbf{k})$ :

$$
H_{A_{1} B_{1}}(\mathbf{k})=\frac{1}{N} \sum_{\mathbf{R}_{A_{1}}} \sum_{\mathbf{R}_{B_{1}}}\left\langle e^{i \mathbf{k} \mathbf{R}_{A_{1}}} \phi\left(\mathbf{r}-\mathbf{R}_{A_{1}}\right)|H| e^{i \mathbf{k} \mathbf{R}_{B_{1}}} \phi\left(\mathbf{r}-\mathbf{R}_{B_{1}}\right)\right\rangle=\gamma_{0} e(\mathbf{k}) .
$$

The matrix element $H_{A_{1} A_{2}}(\mathbf{k})$ yields

$$
\begin{aligned}
H_{A_{1} A_{2}}(\mathbf{k}) & =\frac{1}{N} \sum_{\mathbf{R}_{A_{1}}} \sum_{\mathbf{R}_{A_{2}}}\left\langle e^{i \mathbf{k} \mathbf{R}_{A_{1}}} \phi\left(\mathbf{r}-\mathbf{R}_{A_{1}}\right)|H| e^{i \mathbf{k} \mathbf{R}_{A 2}} \phi\left(\mathbf{r}-\mathbf{R}_{A_{2}}\right)\right\rangle \\
& =\frac{1}{N} \sum_{\mathbf{R}_{A_{1}}} e^{i \mathbf{k} \mathbf{R}_{A_{1}}} e^{-i \mathbf{k} \mathbf{R}_{A}}\left\langle\phi\left(\mathbf{r}-\mathbf{R}_{A_{1}}\right)|H| \phi\left(\mathbf{r}-\mathbf{R}_{A_{2}}\right)\right\rangle=\gamma_{1}
\end{aligned}
$$

with the constant $\left\langle\phi\left(\mathbf{r}-\mathbf{R}_{A_{1}}\right)|H| \phi\left(\mathbf{r}-\mathbf{R}_{A_{2}}\right)\right\rangle=\gamma_{1} \approx 0.35 \mathrm{eV}$ [45 describing the interaction between the $A_{1}$ and $A_{2}$ atoms of the two layers of bilayer graphene [44]. Finally, $H_{A_{1} B_{2}}(\mathbf{k})$ reads

$$
\begin{aligned}
H_{A_{1} B_{2}}(\mathbf{k}) & =\frac{1}{N} \sum_{\mathbf{R}_{A_{1}}} \sum_{\mathbf{R}_{B_{2}}}\left\langle e^{i \mathbf{k} \mathbf{R}_{A_{1}}} \phi\left(\mathbf{r}-\mathbf{R}_{A_{1}}\right)|H| e^{i \mathbf{k} \mathbf{R}_{B_{2}}} \phi\left(\mathbf{r}-\mathbf{R}_{B_{2}}\right)\right\rangle \\
& =\frac{1}{N} \sum_{\mathbf{R}_{A_{1}}} \sum_{i=1}^{3} e^{-i \mathbf{k} \mathbf{b}_{i}}\left\langle\phi\left(\mathbf{r}-\mathbf{R}_{A_{1}}\right)|H| \phi\left(\mathbf{r}-\mathbf{R}_{A_{1}}+\mathbf{b}_{i}\right)\right\rangle=\gamma_{4} e^{*}(\mathbf{k})
\end{aligned}
$$

with $\mathbf{R}_{A_{1}}-\mathbf{R}_{B_{2}}=\mathbf{b}_{i}$. The matrix element $\left\langle\phi\left(\mathbf{r}-\mathbf{R}_{A_{1}}\right)|H| \phi\left(\mathbf{r}-\mathbf{R}_{A_{1}}+\mathbf{b}_{i}\right)\right\rangle$ is equal for all $i$ and labelled as $\gamma_{4} \approx 0.19 \mathrm{eV}$. All other matrix element of Eq. 2.26 are obtained analogously. The overlap matrix elements $S_{i j}=\left\langle\phi_{i} \mid \phi_{j}\right\rangle$ expect for $S_{i i}=1$ are neglected, since their corresponding overlap integrals are considerably small. Inserting the calculated matrix elements into the system of equation (Eq. 2.26), one obtains

$$
\left(\begin{array}{cccc}
\varepsilon_{0}-\varepsilon_{\mathbf{k}} & \gamma_{0} e(\mathbf{k}) & \gamma_{1} & \gamma_{4} e^{*}(\mathbf{k}) \\
\gamma_{0} e^{*}(\mathbf{k}) & \varepsilon_{0}-\varepsilon_{\mathbf{k}} & \gamma_{4} e^{*}(\mathbf{k}) & \gamma_{3} e(\mathbf{k}) \\
\gamma_{1} & \gamma_{4} e(\mathbf{k}) & \varepsilon_{0}-\varepsilon_{\mathbf{k}} & \gamma_{0} e^{*}(\mathbf{k}) \\
\gamma_{4} e(\mathbf{k}) & \gamma_{3} e^{*}(\mathbf{k}) & \gamma_{0} e(\mathbf{k}) & \varepsilon_{0}-\varepsilon_{\mathbf{k}}
\end{array}\right) \cdot\left(\begin{array}{c}
C_{A_{1}}(\mathbf{k}) \\
C_{B_{1}}(\mathbf{k}) \\
C_{A_{2}}(\mathbf{k}) \\
C_{B_{2}}(\mathbf{k})
\end{array}\right)=0
$$




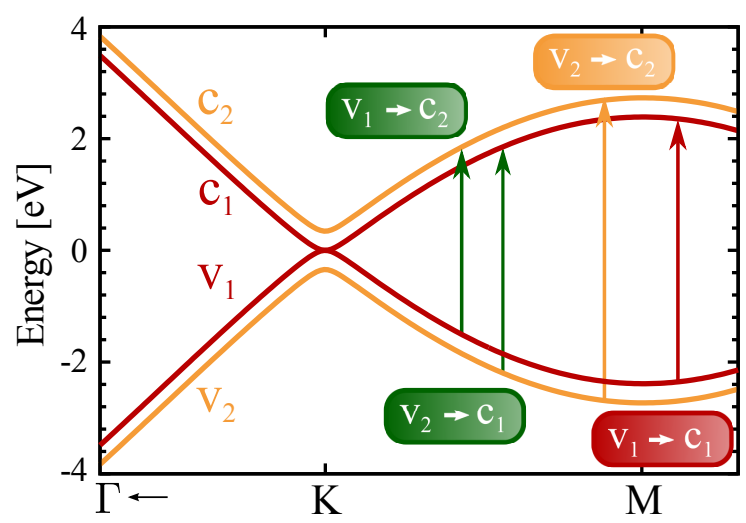

Figure 2.4.: The bandstructure along the high-symmetry $\Gamma K M$ line. The energy dispersion exhibits four parabolic bands, where the valence band $v_{1}$ and the conduction band $c_{1}$ touch at the Dirac point. The energy gap between $v_{2}$ and $c_{2}$ is determined by the tight-binding interaction term $\gamma_{1}$. Figure is taken from [46].

Neglecting the interaction parameters $\gamma_{3}$ and $\gamma_{4}$, the eigenvalues of bilayer graphene results in

$$
\varepsilon_{\mathbf{k} \pm}^{\lambda_{i}}= \pm \frac{1}{2}\left((-1)^{i+1} \gamma_{1}-\sqrt{4|e(\mathbf{k})|^{2} \gamma_{0}^{2}+\gamma_{1}^{2}}\right)
$$

with the band index $i=1,2$ and $(+)$ corresponds to the valence bands $\left(v_{\alpha}\right)$ and $(-)$ to the conduction bands $\left(c_{\alpha}\right)$. The resulting electronic bandstructure of bilayer graphene is illustrated in Fig. 2.4 focusing on the high high-symmetry $\Gamma K M$ line within the two-dimensional Brillouin zone. In contrast to the linear bands in graphene, the energy dispersion exhibits four parabolic bands around the Dirac point, where the valence band $v_{1}$ and the conduction band $c_{1}$ touch. The other pair of bands $\left(v_{2}, c_{2}\right)$ shows an energy gap corresponding to $2 \gamma_{1}=0.72 \mathrm{eV}$. The most important advantage of bilayer graphene lies in the tunability of its bandstructure, which can be achieved by applying an electrical field [11, 12, 47].

\subsection{Matrix elements}

\subsubsection{Optical matrix element}

The optical matrix element determines the strength of coupling between the electron system and an external field in terms of its vector potential $\mathbf{A}(t)$. Within the representation of second quantization the matrix element is given by 41

$$
\mathbf{M}_{\mathbf{k k}^{\prime}}^{\lambda \lambda^{\prime}}=\left\langle\Psi_{\mathbf{k}}^{\lambda}(\mathbf{r})\left|\nabla_{\mathbf{r}}\right| \Psi_{\mathbf{k}^{\prime}}^{\lambda^{\prime}}(\mathbf{r})\right\rangle .
$$



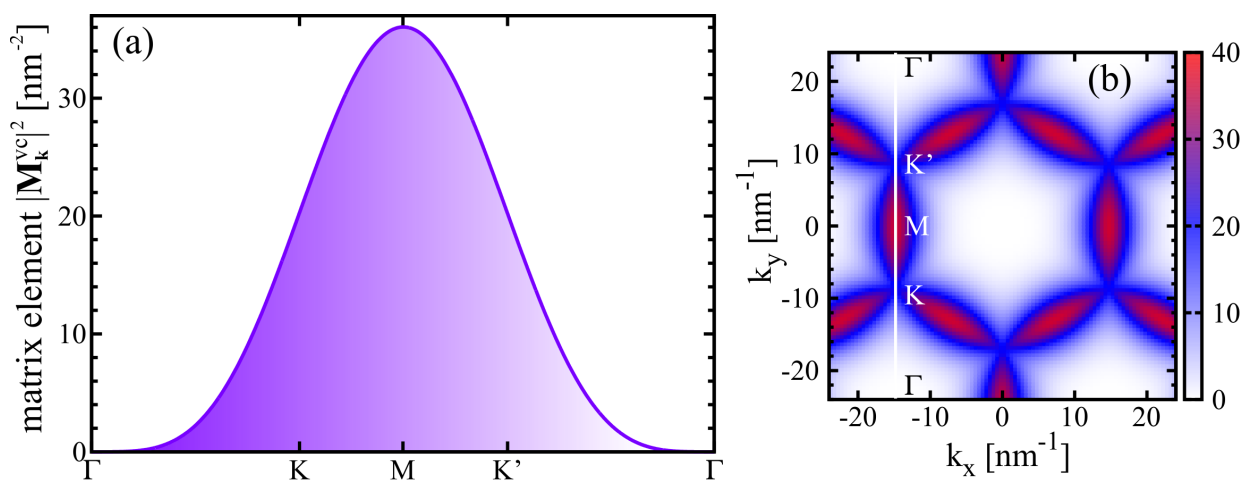

Figure 2.5.: (a) The absolute square of the optical matrix element $\left|\mathbf{M}_{\mathbf{k}}^{v c}\right|^{2}$ is illustrated along the high-symmetry $\Gamma K M$ line within the two-dimensional Brillouin zone of graphene. $\left|\mathbf{M}_{\mathbf{k}}^{v c}\right|^{2}$ shows a maximum at the $M$ point . (b) The contour plots showing the square of the corresponding optical matrix element as a function of $k_{x}$ and $k_{y}$ in the entire Brillouin zone. The white line denotes the high-symmetry $\Gamma K M$ line considered in figure (a).

Applying the tight-binding approach by using the wave function $\Psi_{\mathbf{k}}^{\lambda}(\mathbf{r})$ and exploiting the symmetry of the $2 p_{z}$-orbital [48, 49], the optical matrix element for mono- and bilayer graphene can be calculated by the expression

$\mathbf{M}_{\mathbf{k} \mathbf{k}^{\prime}}^{\lambda \lambda^{\prime}}=\frac{1}{N} \sum_{l, l^{\prime}}^{s} \sum_{\mathbf{R}_{l}, \mathbf{R}_{l^{\prime}}}\left(C_{l}^{\lambda *}(\mathbf{k}) C_{l^{\prime}}^{\lambda^{\prime}}\left(\mathbf{k}^{\prime}\right) e^{-i\left(\mathbf{k} \mathbf{R}_{l}-\mathbf{k}^{\prime} \mathbf{R}_{l}^{\prime}\right)}\left\langle\phi\left(\mathbf{r}-\mathbf{R}_{l}\right)\left|\nabla_{\mathbf{k}}\right| \phi\left(\mathbf{r}-\mathbf{R}_{l^{\prime}}\right)\right\rangle+\right.$ c.c. $)$.

A detailed derivation this equation can be found in appendix A. Including the tight-binding coefficient $C_{l}^{\lambda}$, the specific optical matrix elements for inter- and intraband transitions can be obtained.

Graphene With Eqs. 2.12 and 2.22 the tight-binding coefficients can be written as

$$
C_{A}^{\lambda}(\mathbf{k})=\sigma_{\lambda} \frac{1}{\sqrt{2}} \frac{e(\mathbf{k})}{|e(\mathbf{k})|} \quad \text { and } \quad C_{B}^{\lambda}(\mathbf{k})=\sigma_{\lambda} \frac{1}{\sqrt{2}} .
$$

These coefficients are determined by a complex phase depending on the choice of the used coordinate system [17. Including these coefficients into Eq. 2.34 the optical matrix element for interband $\left(\mathbf{M}_{\mathbf{k}}^{v c}\right)$ and intraband $\left(\mathbf{M}_{\mathbf{k}}^{v v}\right)$ processes are 
given by

$$
\begin{aligned}
& \mathbf{M}_{\mathbf{k}}^{v c}=\frac{M}{|e(\mathbf{k})|\left|b_{1}\right|} \operatorname{Re}\left[e^{*}(\mathbf{k}) \sum_{i=1}^{3} e^{i \mathbf{k} \mathbf{b}_{i}} \mathbf{b}_{i}\right], \\
& \mathbf{M}_{\mathbf{k}}^{v v}=\frac{i M}{|e(\mathbf{k})|\left|b_{1}\right|} \operatorname{Im}\left[e^{*}(\mathbf{k}) \sum_{i=1}^{3} e^{i \mathbf{k} \mathbf{b}_{i}} \mathbf{b}_{i}\right]
\end{aligned}
$$

with the symmetries $\mathbf{M}_{\mathbf{k}}^{v c}=-\mathbf{M}_{\mathbf{k}}^{c v}$ and $\mathbf{M}_{\mathbf{k}}^{v v}=-\mathbf{M}_{\mathbf{k}}^{c c}$ and the constant $M \approx 3,7$ $\mathrm{nm}^{-1}$. Note that the optical matrix element allows only direct optical transition and neglecting the quantum mechanical momentum of the photons.

The optical properties of graphene are mainly determined by the square of the optical interband matrix element $\left|\mathbf{M}_{\mathbf{k}}^{v c}\right|^{2}$, cf. Fig. 2.5. The matrix element vanishes at the $\Gamma$ point, which means that optical transitions are forbidden due to the optical selection rules [41]. Maximal values are found at the $M$ point (saddle point), where the density of states is also very high leading to an absorption peak in the linear optical spectrum of graphene, see chapter 3 . Within the linear regime of the bandstructure the optical matrix element can be further simplified, which is shown in appendix A.1.1.

Bilayer graphene The bilayer graphene tight-binding coefficients $C_{s}^{\lambda}(\mathbf{k})$ can be evaluated by exploiting the normalization of the wave function:

$$
\begin{aligned}
& C_{A_{1}}^{\lambda}(\mathbf{k})=\frac{1}{\beta_{\mathbf{k}}^{\sigma_{i}}}\left(\frac{\gamma_{1}-\sigma_{i} \sqrt{4|e(\mathbf{k})|^{2} \gamma_{0}^{2}+\gamma_{1}^{2}}}{2 e(\mathbf{k}) \gamma_{0}}\right), \\
& C_{B_{1}}^{\lambda}(\mathbf{k})=\sigma_{\lambda} \frac{1}{\beta_{\mathbf{k}}^{\sigma_{i}}}\left(\sigma_{i} \frac{e(\mathbf{k})^{*}}{e(\mathbf{k})}\right) \\
& C_{A_{2}}^{\lambda}(\mathbf{k})=\sigma_{\lambda} \frac{1}{\beta_{\mathbf{k}}^{\sigma_{i}}}\left(\frac{\sigma_{i} \gamma_{1}-\sqrt{4|e(\mathbf{k})|^{2} \gamma_{0}^{2}+\gamma_{1}^{2}}}{2 e(\mathbf{k}) \gamma_{0}}\right), C_{B_{2}}^{\lambda}(\mathbf{k})=\frac{1}{\beta_{\mathbf{k}}^{\sigma_{i}}},
\end{aligned}
$$

where $\sigma_{\lambda}$ equals +1 for $\lambda=v_{i}$ and -1 for $\lambda=c_{i}$. The normalization value is given by

$$
\beta_{\mathbf{k}}^{\sigma_{i}}=\left(4-\frac{4 \gamma_{1}}{\left(\gamma_{1}+\sigma_{i} \sqrt{4|e(\mathbf{k})|^{2} \gamma_{0}^{2}+\gamma_{1}^{2}}\right)}\right)^{\frac{1}{2}}
$$



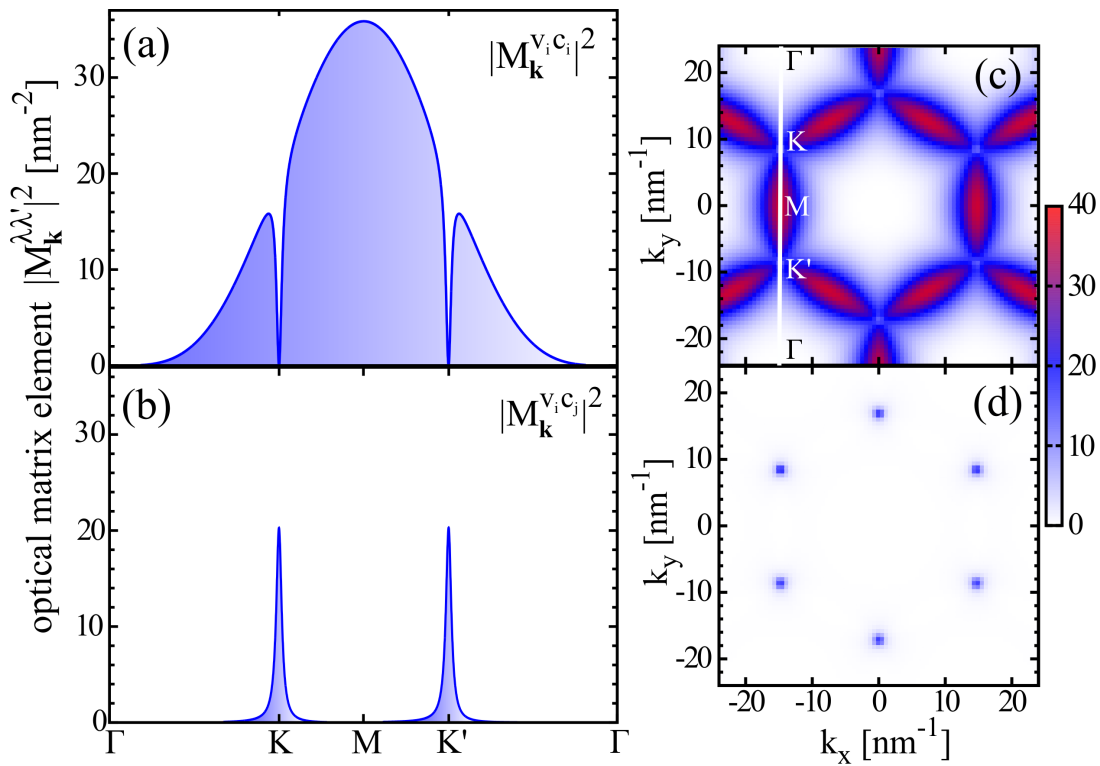

Figure 2.6.: The absolute square of the optical matrix element (a) $\left|\mathbf{M}_{\mathbf{k}}^{v_{i} c_{i}}\right|^{2}$ with $i=1,2$ and (b) $\left|\mathbf{M}_{\mathbf{k}}^{v_{i} c_{j}}\right|^{2}$ with $i \neq j$ is illustrated along the high-symmetry $\Gamma K M$ line within the two-dimensional Brillouin zone of bilayer graphene. While $\left|\mathbf{M}_{\mathbf{k}}^{v_{i} c_{i}}\right|^{2}$ shows a maximum at the $M$ point and vanishes at the $K$ and $K^{\prime}$ points, the coupling element $\left|\mathbf{M}_{\mathrm{k}}^{v_{i} c_{j}}\right|^{2}$ describing optical cross transitions (cp. Fig. 2.4) has only non-zero values at the Dirac points. (c)-(d) The contour plots showing the square of the corresponding optical matrix elements as a function of $k_{x}$ and $k_{y}$ in the entire Brillouin zone. Figure is taken from $\overline{46} \mid$.

with $\sigma_{i}=1,(-1)$ for $i=1,(2)$. Inserting the evaluated coefficients $C_{s}^{\lambda}(\mathbf{k})$ into Eq. (2.34), the optical matrix elements for different transitions read

$$
\begin{aligned}
& \mathbf{M}_{\mathbf{k}}^{v_{i} c_{i}}=-\mathbf{M}_{\mathbf{k}}^{c_{i} v_{i}}=\frac{2 M \gamma_{0}}{\sqrt{4|e(\mathbf{k})|^{2} \gamma_{0}^{2}+\gamma_{1}^{2}}} \operatorname{Re}[\eta(\mathbf{k})], \\
& \mathbf{M}_{\mathbf{k}}^{v_{i} v_{i}}=-\mathbf{M}_{\mathbf{k}}^{c_{i} c_{i}}=-\frac{2 i M \gamma_{0}}{\sqrt{4|e(\mathbf{k})|^{2} \gamma_{0}^{2}+\gamma_{1}^{2}}} \operatorname{Im}[\eta(\mathbf{k})], \\
& \mathbf{M}_{\mathbf{k}}^{\lambda_{1} \lambda_{2}}=-\mathbf{M}_{\mathbf{k}}^{\lambda_{2} \lambda_{1}}=\frac{M \gamma_{1}}{|e(\mathbf{k})|^{2} \gamma_{0}}\left(1+\frac{\gamma_{1}^{2}}{|e(\mathbf{k})|^{2} \gamma_{0}^{2}}\right)^{-\frac{1}{2}} \operatorname{Re}[\eta(\mathbf{k})], \\
& \mathbf{M}_{\mathbf{k}}^{v_{1} c_{2}}=-\mathbf{M}_{\mathbf{k}}^{v_{2} c_{1}}=\frac{i M \gamma_{1}}{|e(\mathbf{k})|^{2} \gamma_{0}}\left(1+\frac{\gamma_{1}^{2}}{|e(\mathbf{k})|^{2} \gamma_{0}^{2}}\right)^{-\frac{1}{2}} \operatorname{Im}[\eta(\mathbf{k})],
\end{aligned}
$$

where $\eta(\mathbf{k})=e^{*}(\mathbf{k}) \sum_{i=1}^{3} e^{i \mathbf{k b}_{i}} \frac{\mathbf{b}_{i}}{\left|\mathbf{b}_{i}\right|}$. The matrix elements fulfil the symmetries $\mathbf{M}_{\mathbf{k}}^{v_{1} c_{2}}=\mathbf{M}_{\mathbf{k}}^{c_{2} v_{1}}=-\mathbf{M}_{\mathbf{k}}^{c_{1} v_{2}}$. 
Figure 2.6 illustrates the square of the optical matrix element as a function of $k_{x}$ and $k_{y}$ along the entire Brillouin zone. Similar to monolayer graphene, the matrix element shows a highly anisotropic coupling with maximal values at the $M$ point. Plotting $\left|\mathbf{M}_{\mathbf{k}}^{v_{i} c_{i}}\right|^{2}$ along the high-symmetry line $\Gamma K M$, we find - in contrast to monolayer graphene - the matrix element goes to zero around the $K$ and $K^{\prime}$ points, whereas the plot of $\left|\mathbf{M}_{\mathbf{k}}^{v_{i} c_{j}}\right|^{2}=\left|\mathbf{M}_{\mathbf{k}}^{v_{i} v_{j}}\right|^{2}$ has only values around the $K$ and $K^{\prime}$ points. At the addressed maxima the density of states is high. As a consequence for the optical spectra two peaks at the $M$ point and one peak at the $K$ point is expected.

\subsubsection{Coulomb matrix element}

The strength of the Coulomb interaction is given by

$$
V_{l_{3} l_{4}}^{l_{1} l_{2}}=\int \mathrm{d} \mathbf{r} \int \mathrm{d} \mathbf{r}^{\prime} \Psi_{l_{1}}^{*}(\mathbf{r}) \Psi_{l_{2}}^{*}\left(\mathbf{r}^{\prime}\right) V_{\text {Coul }}\left(\mathbf{r}-\mathbf{r}^{\prime}\right) \Psi_{l_{4}}(\mathbf{r}) \Psi_{l_{3}}\left(\mathbf{r}^{\prime}\right) .
$$

The derivation of the Coulomb matrix element within the tight binding ansatz is described in the appendix $B$. The resulting matrix elements for graphene and bilayer graphene read

$$
V_{l_{3} l_{4}}^{l_{1} l_{2}}=V_{\mathbf{q}}\left[\left(\frac{q a_{B}}{Z_{\text {eff }}}\right)^{2}+1\right]^{-6} \tilde{\Gamma}_{l_{1}, l_{3}} \tilde{\Gamma}_{l_{4}, l_{2}}^{*} \delta_{\mathbf{q}, \mathbf{k}_{4}-\mathbf{k}_{2}}
$$

with the form factor $\tilde{\Gamma}_{i j}=\sum_{s=A, B} C_{s}^{i *}(\mathbf{k}) C_{s}^{j}(\mathbf{k})$ and $\tilde{\Gamma}_{i j}^{B G}=\sum_{\substack{s=A_{1}, A_{2} \\ B_{1}, B_{2}}} C_{s}^{i *}(\mathbf{k}) C_{s}^{j}(\mathbf{k})$ (bilayer graphene). Here, $q=\left|\mathbf{k}_{3}-\mathbf{k}_{1}\right|$ is the transfer of momentum between the interacting carries, $V_{\mathbf{q}}=\frac{e_{0}^{2}}{2 \varepsilon_{0} L^{2}} \frac{1}{q}$ is the Fourier Transform of the two-dimensional Coulomb potential, $Z_{\text {eff }}$ the effective charge number, and $a_{B}$ the Bohr radius. The delta function ensures the energy conservation for Coulomb-induced scattering processes.

For large momentum distance $q$ the decay of the Coulomb potential is proportional to $q^{-13}$. As a result, the Coulomb interaction is strongly suppressed for large momentum transfers. In contrast, for small momentum transfers $q$ the decay of matrix element scales with $q^{-1}$, since $q \ll Z_{\text {eff }} / a_{B}$. In that case the efficiency of the Coulomb interaction processes depends on the coefficient $\tilde{\Gamma}_{i j}$, which is given within the linear approximation $\mid 50$

$$
\tilde{\Gamma}_{i j}=\frac{1}{2}\left[1+\sigma_{\lambda_{i}} \sigma_{\lambda_{j}} e^{-i\left(\phi_{i}-\phi_{j}\right)}\right]
$$

Here, $\phi_{i}$ describes the orientation angle of the corresponding momentum $\mathbf{k}_{i}$. In the case of intraband processes, the Coulomb matrix element is maximal for parallel 
(a) Inter- and intraband scattering channels

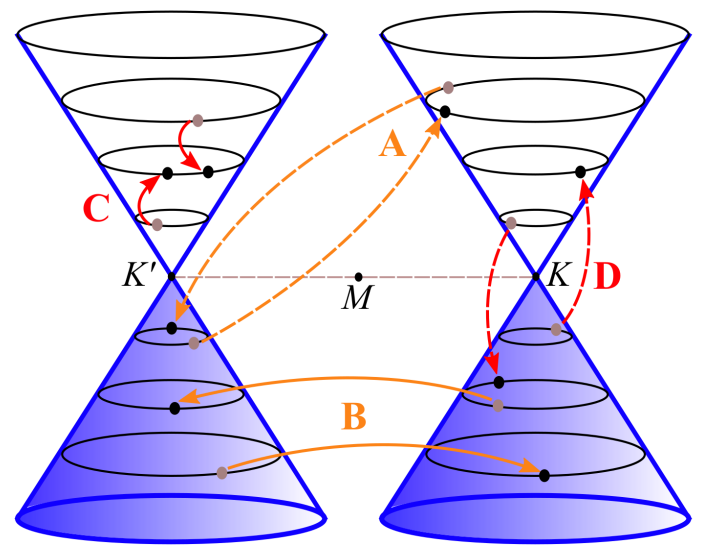

(b) Auger processes

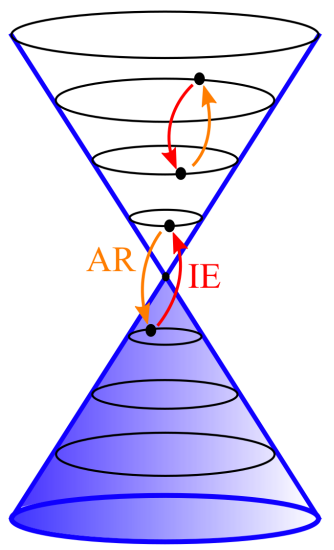

(c) 'Hot' Auger processes

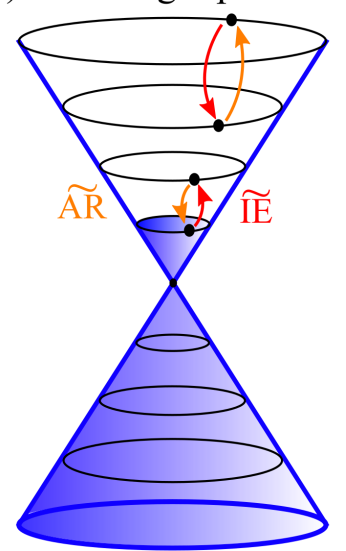

Figure 2.7.: Illustration of the Coulomb-induced scattering channels in graphene.(a) Inter- $(A, D)$ and intraband $(B, C)$ scattering processes. (b) Different Auger-type processes: Auger recombination (AR) and impact excitation (IE). (c) 'Intraband' Auger processes, which take place in a doped system.

scattering processes and zero for anti-parallel scattering, whereas the situation is reserved for interband processes [41,50].

Figure 2.7illustrates the different Coulomb-induced scattering channels in graphene. The Coulomb matrix element $V_{l_{3} l_{4}}^{l_{1} l_{2}}$ contains four band indices, which can be considered as interband, intraband and Auger scattering processes. Auger relaxation processes are remarkable, since they bridge the valence and the conduction band giving rise to carrier multiplication corresponding to an increase of the number of charge carriers in graphene. Two inverse processes have to be distinguished, namely Auger recombination (AR) and its inverse process impact excitation (IE), cp. 2.7(b). The result of IE is an increase of the carrier density, where an electron relaxes to an energetically state within a band inducing the excitation of an electron from valence to the conduction band. In doped graphene, 'intraband' Auger processes bridging the states below and above the Fermi level and resulting in an increase of the number of hot carriers, cp. 2.7(c). Here, the actual number of charge carriers remains unchanged in each band. In section 6.3, a detailed study of Auger-type scattering and carrier multiplication is presented.

\section{Many-particle screening}

The presence of many charge carriers and the substrate screens the Coulomb potential $V_{\mathbf{q}} \rightarrow V_{\mathbf{q}} /\left(\varepsilon_{\mathrm{sub}} \varepsilon(\mathbf{q}, \omega)\right)$. Here, $\varepsilon_{\text {sub }}$ represent an effective dielectric background screening, which is assumed to be an constant value. The intrinsic dynamical screening $\varepsilon(\mathbf{q}, \omega)$ can be self-consistently obtained from higher-order correlations 


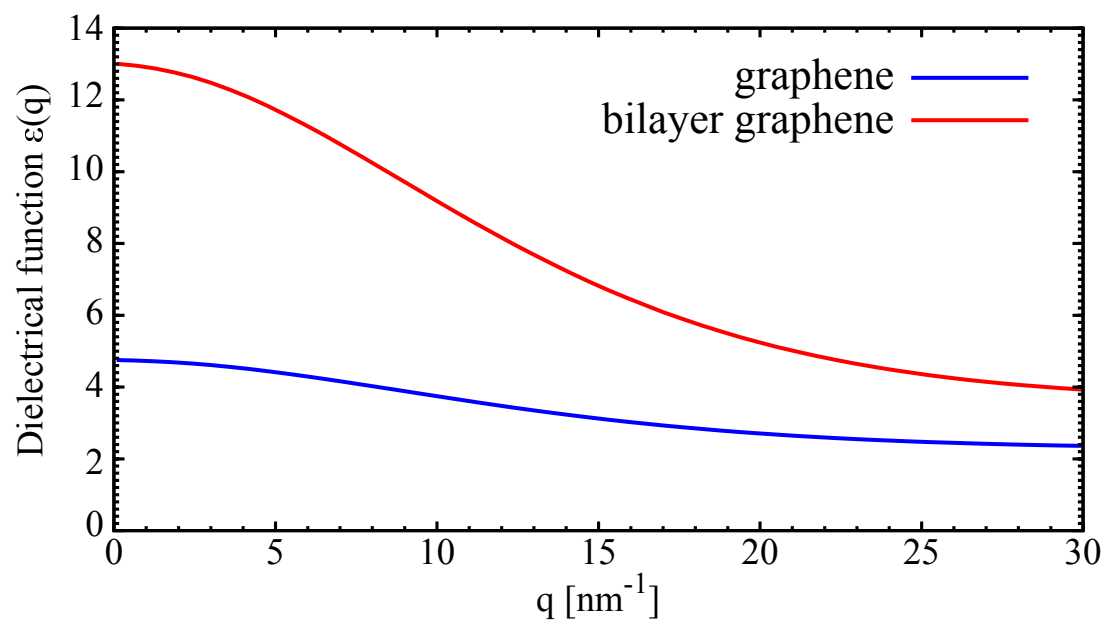

Figure 2.8.: Dielectrical function $\varepsilon(\mathbf{q})$ in the static limit as a function of the momentum transfer for monolayer and bilayer graphene. The figure illustrates the efficient screening for small momentum transfers q. Bilayer graphene shows a three times larger screening for $q \rightarrow 0$.

within the density matrix theory $[51,52$ :

$$
\varepsilon(\mathbf{q}, \omega)=1-2 V_{\mathbf{q}} \sum_{\lambda \lambda^{\prime} \mathbf{k}} \frac{\rho_{\mathbf{k}+\mathbf{q}}^{\lambda^{\prime}}-\rho_{\mathbf{k}}^{\lambda}}{\varepsilon_{\mathbf{k}+\mathbf{q}}^{\lambda^{\prime}}-\varepsilon_{\mathbf{k}}^{\lambda}+\hbar \omega+i \delta_{\mathbf{k}, \mathbf{q}}^{\lambda}}\left|\int d^{3} r \Psi_{\mathbf{k}}^{\lambda *}(\mathbf{r}) e^{i \mathbf{q} \cdot \mathbf{r}} \Psi_{\mathbf{k}-\mathbf{q}}^{\lambda^{\prime}}(\mathbf{r})\right|^{2}
$$

with the bare Coulomb potential $V_{\mathbf{q}}$ and the frequency $\omega=q v_{F} / \hbar$, which represents the energy transfer of one of the two carriers involved in the scattering processes. The momentum dependent broadening $\delta_{\mathbf{k}, \mathbf{q}}$ of the dynamical screening is obtained from higher-order correlations [see appendix D] which becomes important in doped graphene, since it is determined by Coulomb-like scattering rates, which are known to be large in a doped system. Since this many-particleinduced screening is directly influenced by carrier occupations in the conduction and valence bands, doping plays a crucial role and has a significant influence on the ultrafast carrier dynamics in graphene.

Static Screening The static limit of the Lindhart formula is evaluated at $\omega=0$ and $\delta_{\mathbf{k}, \mathbf{q}}^{\lambda}=0$, which is determined in detail in appendix D. Figure 2.8 shows the static dielectric screening $\varepsilon(\mathbf{q})$ along different directions within the Brillouin zone of graphene and bilayer graphene. For $|\mathbf{q}| \approx 0$ the screening function reaches the maximum of 4,7 in graphene and 13 in bilayer graphene, respectively. The screening becomes for increasing momentum transfer $\mathbf{q}$ less efficient until it reaches the value 1 for very large momentum $\mathbf{q}$. In bilayer graphene the screening is almost 
three times larger than in the monolayer case for small momentum transfers, since more carriers are available due to the additional bands.

\subsubsection{Electron-phonon matrix elements}

Optical phonons The phonon dispersion is illustrated in Fig. 2.9 and characterized by different phonon modes. They are divided into acoustic phonons $\left(\Gamma_{L A}\right)$, and optical phonon $\left(\Gamma_{L O}, \Gamma_{T O}, K_{T O}\right)$. In the case of the optical phonons, the dispersion at the $\Gamma$ and $K$ point is approximately constant with the values $\hbar \omega_{\Gamma_{L O}}=198 \mathrm{mev}, \hbar \omega_{\Gamma_{T O}}=192 \mathrm{mev}$, and $\hbar \omega_{K_{T O}}=162 \mathrm{mev}$ [53. The momentum transfer during a scattering processes of a carrier located within the Dirac cone could be either small (intravalley process) or very large with $q \approx 4 \pi /\left(\sqrt{3} a_{0}\right)$ (intervalley scattering between $K$ and $K^{\prime}$ points), cp. Fig. 2.9(b). The electron-phonon coupling elements, which determines the symmetry and the probability of each process, are given for the $\Gamma_{L O}, \Gamma_{T O}$ and $K$ modes by [40]

$$
\begin{aligned}
& \left|g_{\mathbf{q}, \Gamma_{L O}}^{\mathbf{k}, \lambda \lambda^{\prime}}\right|^{2}=\left|g_{\mathbf{q}, \Gamma_{T O}}^{\mathbf{k}, \lambda \lambda}\right|^{2}=\frac{1}{N} \tilde{g}_{\Gamma}^{2}\left[1+\cos \left(\varphi+\varphi^{\prime}\right)\right], \\
& \left|g_{\mathbf{q}, \Gamma_{T O}}^{\mathbf{k}, \lambda \lambda^{\prime}}\right|^{2}=\left|g_{\mathbf{q}, \Gamma_{L O}}^{\mathbf{k}, \lambda \lambda}\right|^{2}=\frac{1}{N} \tilde{g}_{\Gamma}^{2}\left[1-\cos \left(\varphi+\varphi^{\prime}\right)\right], \\
& \left|g_{\mathbf{q}, K_{T O}}^{\mathbf{k}, \lambda \lambda \lambda^{\prime}}\right|^{2}=\frac{1}{N} \tilde{g}_{K}^{2}\left[1-\sigma_{\lambda \lambda^{\prime}} \cos \left(\varphi^{\prime \prime}\right)\right],
\end{aligned}
$$

with $\tilde{g}_{\Gamma}^{2}=0.0405 \mathrm{eV}^{2}, \tilde{g}_{K}^{2}=0.0994 \mathrm{eV}^{2}$ and the angles $\varphi, \varphi^{\prime}$ and $\varphi^{\prime \prime}$ between the wave vectors of the involved carriers and phonons, cp. the inset of Fig. 2.9(c). The electron-phonon matrix elements for the optical modes do not directly depend on the phonon momentum $q$, but they exhibit a angle dependence for each mode. The longitudinal interband and transversal intraband coupling is most efficient for $\varphi+\varphi^{\prime}=0$ and minimal for $\varphi+\varphi^{\prime}= \pm \pi$, cp. Fig. 2.9(c). The situation for the longitudinal intraband and transversal interband matrix elements is reserved. The electron-phonon matrix element for $K$ phonons is more than twice as large as for the $\Gamma$ modes. The interband $K_{T O}$ mode is maximal for $\mathbf{k}$ perpendicular to $\mathbf{k}+\mathbf{q}$ and vanishes for $\varphi^{\prime \prime}=0$.

Acoustic phonons The dispersion of acoustic phonons is assumed to be linear in the long-wavelength region with $\hbar \omega_{\mathbf{q}}^{j}=\hbar \nu_{\Gamma_{L A}}|\mathbf{q}|$, cp. Figure 2.9(a). Only the strongest $\Gamma_{L A}$ phonon mode with $\nu_{\Gamma_{L A}}=2 \times 10^{4} \mathrm{~ms}^{-1}$ is considered. The corresponding matrix element for the acoustic mode is applied from Das Sarma et al. [55] and given by

$$
\left|g_{\mathbf{q}, \Gamma_{L A}}^{\mathbf{k}, \lambda \lambda^{\prime}}\right|^{2}=\frac{1}{L^{2}} \frac{q D^{2} \hbar}{2 M \nu_{\Gamma_{L A}}}\left[1-\cos \left(\varphi^{\prime \prime}\right)\right]
$$


(a)

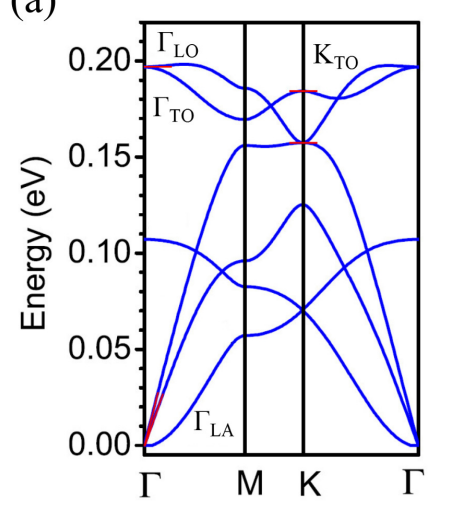

(b)

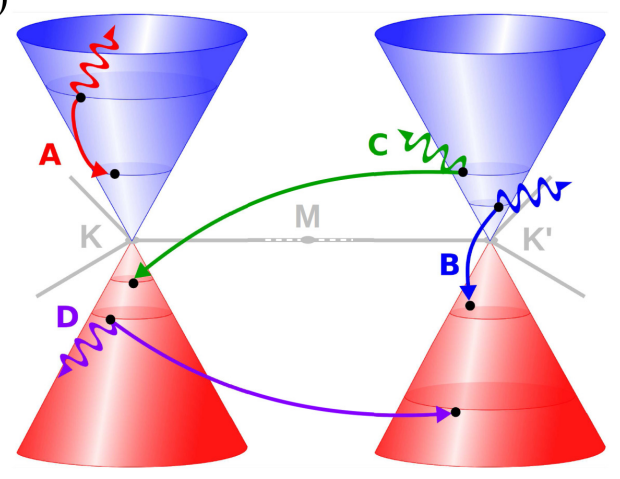

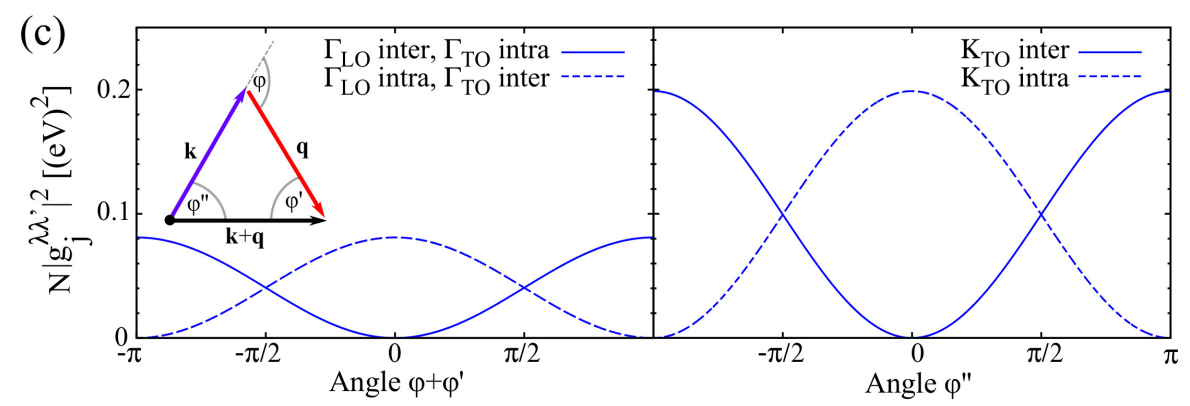

Figure 2.9.: (a)Phonon dispersion of graphite along the high-symmetry line $\Gamma M K \Gamma$, where two optical (LO,TO) and one acoustic $(L A)$ modes are illustrated. The red lines describe the approximately linear dispersion for acoustic and constant energy dispersion for optical modes. Figure is taken from [54]. (b) Phonon-induced intra- and interband as well as intra- and intervalley scattering processes (emission). The intervalley scattering (C) and (D) is induced by the $K$-mode, whereas the $\Gamma$-mode induces intra- $(\mathrm{A})$ and interband scattering (B). Figure is adopted from [17]. (c) Angle dependence of the electron-phonon matrix elements for the optical modes $\Gamma_{L O}, \Gamma_{T O}$ and $K_{T O}$ including the definition of the phonon-induced scattering angles.

where $D=16 \mathrm{eV}$ is the deformation potential and $M=7.6 \times 10^{-8} \mathrm{gcm}^{-2}$ the mass density of graphene [55]. In contrast to the optical modes, the acoustic phonon scattering depends on the phonon momentum $q$ allowing for continuous energy transfer. Since $\nu_{\Gamma_{L A}}$ is smaller than the Fermi velocity $v_{F}$, the absorption and emission of an acoustic phonon occurs only as an intraband processes 55. 


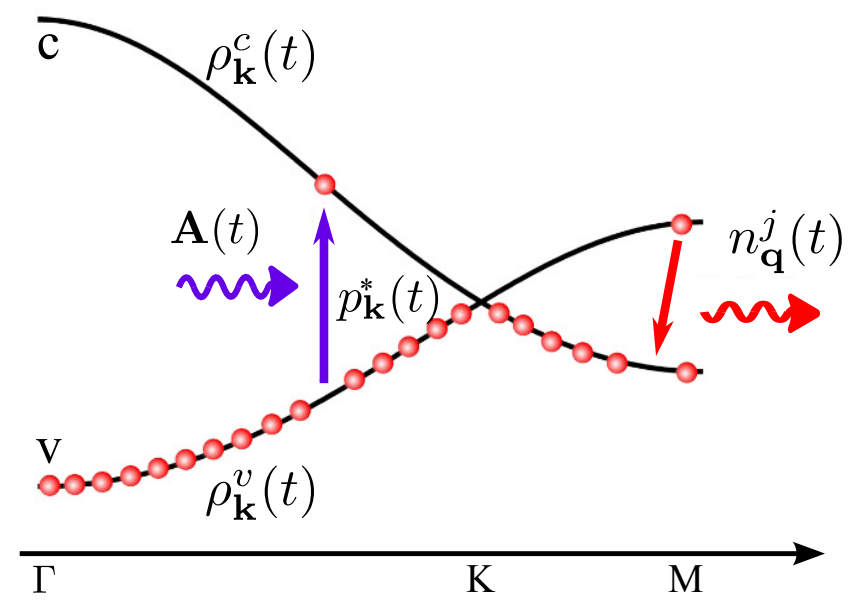

Figure 2.10.: Illustration of the microscopic polarization $p_{\mathbf{k}}$, the occupation probability $\rho_{\mathbf{k}}^{\lambda}$ and the phonon occupation $n_{\mathbf{q}}^{j}$ within the band structure of graphene. The vector potential $\mathbf{A}(t)$ describes the optical excitation by lifting electrons from the valence into the conduction band.

\subsection{Microscopic Bloch equation}

With the Hamilton operator $H$ (Eq. 2.1) the microscopic access to the temporal evolution of an arbitrary operator quantity $x(t)$ within the Heisenberg equation of motion is given by $\mid 51$

$$
i \hbar \frac{d}{d t} x(t)=[x(t), H]_{-} .
$$

The focus of this work is the carrier and phonon dynamics in a non-equilibrium, where the relevant time- and momentum dependent quantities are the carrier occupation $\rho_{\mathbf{k}}^{\lambda}(t)=\left\langle a_{\mathbf{k}}^{\lambda \dagger} a_{\mathbf{k}}^{\lambda}\right\rangle(t)$, the microscopic polarization $p_{\mathbf{k}}(t)=\left\langle a_{\mathbf{k}}^{\lambda \dagger} a_{\mathbf{k}}^{\lambda^{\prime}}\right\rangle(t)$, which is a measure for the transition probability between the valence and conduction band, and the phonon population $n_{\mathbf{k}}^{j}(t)=\left\langle b_{\mathbf{q}}^{j \dagger} b_{\mathbf{q}}^{j}\right\rangle(t)$. Figure 2.10 illustrates these microscopic quantities within the electronic bandstructure of graphene. An optical pulse described by the vector potential $\mathbf{A}(t)$ perturbs the carrier system resulting in a generation of a non-equilibrium carrier distribution by lifting electrons from the valence into the conduction band. The optical excitation and the electron-electron and electron-phonon scattering dynamics changes the occupation probabilities $\rho_{\mathbf{k}}^{\lambda}$ as well as the phonon occupation $n_{\mathbf{k}}^{j}$. Note that in bilayer graphene the polarization has two band indices $\left(p_{\mathbf{k}}^{\lambda_{i} \lambda_{j}^{\prime}}\right)$ representing different possible transitions due to the two valance and conduction bands, for instance the transition $v_{1} \rightarrow c_{2}$. Applying the Heisenberg equation of motion and exploiting the fundamental commutator relations for fermionic and bosonic operators the resulting microscopic 
Bloch equations read

$\dot{\rho}_{\mathbf{k}}^{\lambda}(t)=\sigma_{\lambda} 2 \operatorname{Im}\left[\Omega_{\mathbf{k}}^{v c *}(t) p_{\mathbf{k}}(t)\right]+\left.\dot{\rho}_{\mathbf{k}}^{\lambda}(t)\right|_{\mathrm{HF}+\text { scat }}$,

$\dot{p}_{\mathbf{k}}(t)=\left[i \omega_{\mathbf{k}}^{v c}+\Omega_{\mathbf{k}}^{v v}(t)-\Omega_{\mathbf{k}}^{c c}(t)\right] p_{\mathbf{k}}(t)+i \Omega_{\mathbf{k}}^{v c}(t)\left[\rho_{\mathbf{k}}^{v}(t)-\rho_{\mathbf{k}}^{c}(t)\right]+\left.\dot{p}_{\mathbf{k}}(t)\right|_{\mathrm{HF}+\text { scat }}$,

$\dot{n}_{\mathbf{q}}^{j}(t)=-\gamma_{\mathrm{ph}}\left[n_{\mathbf{q}}^{j}(t)-n_{B}^{j}\right]+\left.\dot{n}_{\mathbf{q}}^{j}(t)\right|_{\mathrm{HF}+\mathrm{scat}}$.

Here, $\omega_{\mathbf{k}}^{v c}=\frac{1}{\hbar}\left(\varepsilon_{\mathbf{k}}^{v}-\varepsilon_{\mathbf{k}}^{c}\right)$ is the optical transition frequency within the linear bandstructure $\varepsilon_{\mathbf{k}}^{\lambda}$ of graphene near the Dirac point. 41] The carrier-light coupling is determined by the $\Omega_{\mathbf{k}}^{\lambda \lambda^{\prime}}(t)=\frac{i e_{0}}{m_{0}} \mathbf{M}_{\mathbf{k}}^{\lambda \lambda^{\prime}} \cdot \mathbf{A}(t)$ with the optical matrix element $\mathbf{M}_{\mathbf{k}}^{\lambda \lambda^{\prime}}$ and vector potential $\mathbf{A}(t)$ of the exciting pulse [17]. $\Omega_{\mathbf{k}}^{v c}(t)$ is the Rabi frequency and $\Omega_{\mathbf{k}}^{\lambda \lambda}(t)$ accounts for intraband transitions $[56]$. The finite phonon lifetime is considered by a coupling to a phonon bath $n_{B}^{j}$ (which is given by the Bose-Einstein distribution at room temperature) at ambient temperature with the experimental determined phonon lifetime $\gamma_{\mathrm{ph}}[17$.

A finite doping breaks the symmetry between the valence and conduction band around the Dirac point, cp. Fig. 6.5 in chapter 6. As a result, the occupation probability of electrons $\rho_{\mathbf{k}}^{e}=\rho_{\mathbf{k}}^{c}$ and of holes $\rho_{\mathbf{k}}^{h}=1-\rho_{\mathbf{k}}^{v}$ needs to be treated separately. As initial condition, a Fermi distribution $\left.\rho_{\mathbf{k}}^{(h, e)}=1 /\left[\exp \left(\left(\varepsilon_{\mathbf{k}}^{\lambda} \pm \varepsilon_{F}\right) / k_{B} T\right)\right)+1\right]$ is assumed with the Fermi level $\varepsilon_{F}$, and the temperature $T$, where + stands for the hole and - for the electron occupation.

The many-particle interactions in Eqs. 2.53 2.55 can be separated in a HartreeFock (HF) and a scattering (scat) part [41. These interactions couple the dynamics of single-particle elements to higher-order terms describing the correlation between carriers. The temporal evolution for the appearing two-particle quantities depends on three-particle terms, which couple to four-particle quantities, and so on. To obtain a finite set of equations, the infinite hierarchy needs to be systematically truncated at some level by exploiting the correlation expansion [51]. The $\mathrm{n}$-th order expectation value is factorized into all possible permutations of correlated expectation values of the same and lower order correlations:

$$
\begin{aligned}
\left\langle x_{a}\right\rangle= & \left\langle x_{a}\right\rangle^{c} \\
\left\langle x_{a} x_{b}\right\rangle= & \left\langle x_{a} x_{b}\right\rangle^{c}+\left\langle x_{a}\right\rangle^{c}\left\langle x_{b}\right\rangle^{c} \\
\left\langle x_{a} x_{b} x_{c}\right\rangle= & \left\langle x_{a} x_{b} x_{c}\right\rangle^{c}+(-1)^{P(a b c)}\left\langle x_{a}\right\rangle^{c}\left\langle x_{b} x_{c}\right\rangle^{c}+(-1)^{P(c a b)}\left\langle x_{c}\right\rangle^{c}\left\langle x_{a} x_{b}\right\rangle^{c} \\
& +(-1)^{P(b c a)}\left\langle x_{b}\right\rangle^{c}\left\langle x_{c} x_{a}\right\rangle^{c}+\left\langle x_{a}\right\rangle^{c}\left\langle x_{b}\right\rangle^{c}\left\langle x_{c}\right\rangle^{c} .
\end{aligned}
$$

Here, $P($.$) is the number of permutations of fermion operators with respect to the$ uncorrelated expectation value. In this thesis, only contributions from a certain order are considered, where the higher-order terms are assumed to be negligibly small. This factorization technique leads to a closed set of equations for the singleparticle elements. 


\subsubsection{Hartree-Fock approximation}

In this section, the Hartree-Fock approximation applied to the Coulomb interaction will be discussed. This approximation already describes well the formation of excitons [17, 57 -59], which mainly describe the excitonic spectra of graphene and bilayer graphene, see chapter 3 . Assuming $\rho_{\mathbf{k}}^{v}=1$ and $\rho_{\mathbf{k}}^{c}=0$ (linear optics), the Bloch equations for graphene read within the Hartree-Fock approximation:

$$
\left.\dot{p}_{\mathbf{k}}(t)\right|_{\mathrm{HF}}=\frac{i}{\hbar} \sum_{\mathbf{k}^{\prime}}\left[\left(V_{\mathbf{k} v, \mathbf{k}^{\prime} c}^{\mathbf{k}^{\prime} v, \mathbf{k}}-V_{\mathbf{k} v, \mathbf{k}^{\prime} c}^{\mathbf{k}^{\prime} v, \mathbf{k} c}\right) p_{\mathbf{k}}(t)+\left(V_{\mathbf{k} v, \mathbf{k}^{\prime} c}^{\mathbf{k}^{\prime} v, \mathbf{k}} p_{\mathbf{k}^{\prime}}(t)+V_{\mathbf{k} v, \mathbf{k}^{\prime} v}^{\mathbf{k}^{\prime} c, \mathbf{k}} p_{\mathbf{k}^{\prime}}^{*}(t)\right)\right] .
$$

The resulting bilayer graphene Bloch equations is given by

$$
\begin{aligned}
\left.\dot{p}_{\mathbf{k}, B G}^{\lambda_{i} \lambda_{j}^{\prime}}(t)\right|_{\mathrm{HF}}=\frac{i}{\hbar} \sum_{\mathbf{k}^{\prime}}[ & \left(-V_{\mathbf{k} \lambda_{i}, \mathbf{k}^{\prime} \lambda_{i}}^{\mathbf{k}^{\prime} \lambda_{i}, \mathbf{k} \lambda_{i}}-V_{\mathbf{k} \lambda_{i}, \mathbf{k}^{\prime} \lambda_{j}}^{\mathbf{k}^{\prime} \lambda_{j}, \mathbf{k} \lambda_{i}}+V_{\mathbf{k} \lambda_{j}^{\prime}, \mathbf{k}^{\prime} \lambda_{i}}^{\mathbf{k}^{\prime} \lambda_{i}, \mathbf{k} \lambda_{j}^{\prime}}+V_{\mathbf{k} \lambda_{j}^{\prime}, \mathbf{k}^{\prime} \lambda_{j}}^{\mathbf{k}^{\prime} \lambda_{j}, \mathbf{k} \lambda_{j}^{\prime}}\right) p_{\mathbf{k}}^{\lambda_{i} \lambda_{j}^{\prime}}(t) \\
& \left.+V_{\mathbf{k} \lambda_{i}, \mathbf{k}^{\prime} \lambda_{j}^{\prime}}^{\mathbf{k}^{\prime} \lambda_{i}, \mathbf{k} \lambda_{\mathbf{k}^{\prime}}^{\prime}} p^{\lambda_{i} \lambda_{j}^{\prime}}(t)+V_{\mathbf{k} \lambda_{i}, \mathbf{k}^{\prime} \lambda_{i}}^{\mathbf{k}^{\prime} \lambda_{j}^{\prime}, \mathbf{k} \lambda_{j}^{\prime}} p_{\mathbf{k}^{\prime}}^{\lambda_{i} \lambda_{j}^{\prime} *}(t)\right] .
\end{aligned}
$$

There are two different contributions to the dynamics of the microscopic polarization from the Coulomb-induced Bloch equation. In the case of graphene, the term proportional to $p_{\mathbf{k}}(t)$ describes the repulsive electron-electron part of the Coulomb interaction and results in an energy renormalization. Since these renormalization terms are always larger than zero a blue-shift of the absorption spectrum is expected. The remaining terms in 2.57 are proportional to $p_{\mathbf{k}^{\prime}}$ and represent a renormalization of the Rabi frequency $\Omega_{\mathbf{k}}^{\lambda \lambda}(t)$. They correspond to the attractive electron-hole part of the Coulomb interaction and lead to a momentum-dependent redistribution of the oscillator strength [17,41,59].

The Hartree-Fock contribution will be only considered for the excitonic absorption spectra in chapter 3, since their main influence is around the $M$ point and negligible near the Dirac point [17].

\subsubsection{Second-order Born-Markov approximation}

To obtain the scattering parts of the polarization $\left.p_{\mathbf{k}}\right|_{\text {scat }}$, the occupation $\left.\rho_{\mathbf{k}}^{\lambda}\right|_{\text {scat }}$, and the phonon population $\left.n_{\mathbf{q}}^{j}\right|_{\text {scat }}$, carrier-carrier and carrier-phonon scattering processes have to be considered within the second-order Born-Markov approximation. The resulting microscopic Boltzmann-like kinetic equation for the carrier occupation probabilities read

$$
\left.\dot{\rho}_{l}(t)\right|_{\mathrm{scat}}=\left[\Gamma_{l}^{\mathrm{in}, c p}(t)+\Gamma_{l}^{\mathrm{in}, c c}(t)\right]\left(1-\rho_{l}(t)\right)+\left[\Gamma_{l}^{\mathrm{out}, c p}(t)+\Gamma_{l}^{\mathrm{out}, c c}(t)\right] \rho_{l}(t)
$$

with the the time- and momentum-dependent phonon-induced $\Gamma_{l}^{\text {in,out,cp }}$ and carriercarrier $\Gamma_{l}^{\text {in,out, } c c}$ scattering rates. These equations are obtained by applying the 
Markov approximation, which neglects quantum mechanical memory effects. To give a short example for the Markov approximation, a differential equation of the form

$$
\dot{x}(t)=(i \Delta \omega-\gamma) x(t)+i Q(t)
$$

appears in the derivation of the carrier-carrier and carrier-phonon scattering contributions. Here, $\Delta \omega$ is the frequency difference of all involved states, $\gamma$ a damping constant, and $Q(t)$ a scattering kernel, which contains the integrals over all scattering and dephasing contributions. A formal integration of this inhomogeneous differential equations yields

$$
x(t)=i \int_{0}^{\infty} \mathrm{d} s e^{(i \Delta \omega-\gamma) s} Q(t-s) .
$$

Assuming that the scattering kernel $Q(t-s)$ is a slowly varying function compared to the oscillation $e^{-i(\Delta \tilde{\omega}) s}$, the memory kernel of $Q(t-s)$ can be neglected via $\left.Q(t-s)=\tilde{Q}(t) e^{-i(\Delta \tilde{\omega}-\Delta \omega}\right) s$, where the quantum numbers of $\Delta \tilde{\omega}$ stem from the free energy part of the scattering kernel. This assumption leads to a simple analytic solution of Eq. 2.60.

$$
x(t)=\tilde{Q}(t)\left[i \frac{\gamma}{\Delta \tilde{\omega}^{2}+\gamma^{2}}-\mathcal{P}\left(\frac{\Delta \tilde{\omega}}{\Delta \tilde{\omega}^{2}+\gamma^{2}}\right)\right]
$$

with the principal value $\mathcal{P}($.$) . The influence of the principal value on the carrier$ dynamics in graphene is known to be small and will be neglected in the following. Finally, for a vanishing $\gamma$ the integral in Eq. 2.61 yields

$$
x(t)=i \pi \tilde{Q}(t) \delta(\Delta \tilde{\omega})
$$

where the delta function represents the energy conservation.

In the next two sections, the specific carrier-carrier as well as carrier-phonon scattering rates are presented. In addition, the phonon- and Coulomb-induced dynamics of the microscopic polarization is presented. A detailed derivation of the following expressions can be found in the appendix $\mathrm{C}$.

\section{Carrier-Carrier scattering rates}

The in- and out-scattering rates for the Coulomb-induced carrier dynamics are given by

$$
\begin{aligned}
\Gamma_{l}^{\mathrm{in}, c c}(t) & =\frac{2 \pi}{\hbar} \sum_{a b c} V_{b c}^{l a} \tilde{V}_{b c}^{l a}\left[1-\rho_{a}\right] \rho_{b} \rho_{c} \delta\left(\varepsilon_{l}+\varepsilon_{a}-\varepsilon_{b}-\varepsilon_{c}\right), \\
\Gamma_{l}^{\mathrm{out}, c c}(t) & =\frac{2 \pi}{\hbar} \sum_{a b c} V_{b c}^{l a} \tilde{V}_{b c}^{l a} \rho_{a}\left[1-\rho_{b}\right]\left[1-\rho_{c}\right] \delta\left(\varepsilon_{l}+\varepsilon_{a}-\varepsilon_{b}-\varepsilon_{c}\right)
\end{aligned}
$$


with $\tilde{V}_{b c}^{l a}=2 V_{b c}^{l a *}-V_{b c}^{a l *}$. The delta function in the scattering rates resulting from the Markov approximation assures the energy conservation. The increase of the occupation in the state $l=(\lambda, \mathbf{k})$ is given by to the product of the Pauli blocking term $\left(1-\rho_{l}\right)$ (Eq. 2.59) and the in-scattering rate. On the other hand, the decrease of the occupation is proportional to the occupation $\rho_{l}$ in Eq. 2.59 and the out-scattering rate.

\section{Carrier-Phonon scattering rates}

The in- and out-scattering rates of the phonon-induced carrier dynamics read

$$
\begin{aligned}
\Gamma_{\lambda, \mathbf{k}}^{\mathrm{in}, c p}=\frac{2 \pi}{\hbar} \sum_{\lambda^{\prime} j \mathbf{q}} & {\left[\left|g_{\mathbf{k}, \mathbf{q}}^{\lambda^{\prime} \lambda j}\right|^{2} \rho_{\mathbf{k}-\mathbf{q}}^{\lambda^{\prime}} n_{\mathbf{q}}^{j} \delta\left(\Delta \varepsilon_{\mathbf{k}, \mathbf{q}}^{\lambda \lambda^{\prime}}\right)+\left|g_{\mathbf{k}+\mathbf{q}, \mathbf{q}}^{\lambda^{\prime} \lambda j}\right|^{2} \rho_{\mathbf{k}+\mathbf{q}}^{\lambda^{\prime}}\left(n_{\mathbf{q}}^{j}+1\right) \delta\left(\Delta \varepsilon_{\mathbf{k}+\mathbf{q}, \mathbf{q}}^{\lambda^{\prime} \lambda}\right)\right] } \\
\Gamma_{\lambda, \mathbf{k}}^{\text {out }, c p}=\frac{2 \pi}{\hbar} \sum_{\lambda^{\prime} j \mathbf{q}} & {\left[\left|g_{\mathbf{k}, \mathbf{q}}^{\lambda^{\prime} \lambda j}\right|^{2}\left[1-\rho_{\mathbf{k}-\mathbf{q}}^{\lambda^{\prime}}\right]\left[n_{\mathbf{q}}^{j}+1\right] \delta\left(\Delta \varepsilon_{\mathbf{k}, \mathbf{q}}^{\lambda \lambda^{\prime}}\right)\right.} \\
& \left.+\left|g_{\mathbf{k}+\mathbf{q}, \mathbf{q}}^{\lambda^{\prime} \lambda j}\right|^{2}\left[1-\rho_{\mathbf{k}+\mathbf{q}}^{\lambda^{\prime}}\right]\left(n_{\mathbf{q}}^{j}+1\right) \delta\left(\Delta \varepsilon_{\mathbf{k}+\mathbf{q}, \mathbf{q}}^{\lambda^{\prime} \lambda}\right)\right]
\end{aligned}
$$

with the energy conversation condition $\Delta \varepsilon_{\mathbf{k}, \mathbf{q}}^{\lambda \lambda^{\prime}}=\varepsilon_{\mathbf{k}}^{\lambda}-\varepsilon_{\mathbf{k}-\mathbf{q}}^{\lambda^{\prime}}-\hbar \omega_{\mathbf{q}}^{j}$. The absorption of a phonon depends on the phonon occupation $n_{\mathbf{q}}^{j}$, while the phonon emission is proportional to $n_{\mathbf{q}}^{j}+1$, which can always take place.

The scattering part of the phonon dynamics is also described by a Boltzmann-like equation:

$$
\left.\dot{n}_{\mathbf{q}}^{j}(t)\right|_{\text {scat }}=\Gamma_{\mathbf{q}}^{j, \mathrm{em}}(t)\left[n_{\mathbf{q}}^{j}(t)+1\right]-\Gamma_{\mathbf{q}}^{j, \text { abs }}(t) n_{\mathbf{q}}^{j}(t)
$$

with the phonon emission and absorption rates

$$
\begin{aligned}
& \Gamma_{\mathbf{q}}^{\mathrm{em}, j}(t)=\frac{2 \pi}{\hbar} \sum_{\lambda \lambda^{\prime} \mathbf{k}}\left|g_{\mathbf{k}, \mathbf{q}}^{\lambda \lambda^{\prime} j}\right|^{2} \rho_{\mathbf{k}}^{\lambda}\left[1-\rho_{\mathbf{k}-\mathbf{q}}^{\lambda^{\prime}}\right] \Delta \varepsilon_{\mathbf{k}, \mathbf{q}}^{\lambda \lambda^{\prime}}, \\
& \Gamma_{\mathbf{q}}^{\mathrm{abs}, j}(t)=\frac{2 \pi}{\hbar} \sum_{\lambda \lambda^{\prime} \mathbf{k}}\left|g_{\mathbf{k}, \mathbf{q}}^{\lambda \lambda^{\prime} j}\right|^{2}\left[1-\rho_{\mathbf{k}}^{\lambda}\right] \rho_{\mathbf{k}-\mathbf{q}}^{\lambda^{\prime}} \Delta \varepsilon_{\mathbf{k}, \mathbf{q}}^{\lambda \lambda^{\prime}}
\end{aligned}
$$

The efficiency of the phonon emission and absorption processes depend on the carrier-phonon coupling element $\left|g_{\mathbf{k}, \mathbf{q}}^{\lambda \lambda^{\prime} j}\right|^{2}$ (see section 2.3.3 as well as on the occupation of the initial state $(\lambda)$ and the final state $\left(\lambda^{\prime}\right)$. The microscopic treatment of the phonon dynamics beyond the bath approximation is very important, since the scattering via phonons can be very efficient (hot phonon effect) resulting in a slower relaxation dynamics 17,60 .

The finite phonon lifetime is considered by a coupling to a phonon bath $n_{B}^{j}$ at ambient temperature with the experimentally determined phonon lifetime $\gamma_{\mathrm{ph}}$ 61:

$$
\left.\dot{n}_{\mathbf{q}}^{j}(t)\right|_{\text {scat }}=\Gamma_{\mathbf{q}}^{\mathrm{em}, j}(t)\left[n_{\mathbf{q}}^{j}(t)+1\right]-\Gamma_{\mathbf{q}}^{\mathrm{abs}, j}(t) n_{\mathbf{q}}^{j}(t) .
$$




\section{Many-Particle Dephasing}

The phonon- and Coulomb-induced dynamics of the microscopic polarization $p_{\mathbf{k}}(t)$ is given by

$$
\left.\dot{p}_{\mathbf{k}}(t)\right|_{\text {scat }}=-\gamma_{\mathbf{k}}(t) p_{\mathbf{k}}(t)+\mathcal{U}_{\mathbf{k}}(t)
$$

with the diagonal dephasing $\gamma_{\mathbf{k}}(t)$ and nondiagonal part $\mathcal{U}_{\mathbf{k}}(t)$. The many-particle interactions $\gamma_{\mathbf{k}}$ and $\mathcal{U}_{\mathbf{k}}$ read

$$
\begin{aligned}
\gamma_{\mathbf{k}}(t) & =\frac{1}{2} \sum_{\lambda}\left[\Gamma_{\lambda, \mathbf{k}}^{\mathrm{in}, c p+c c}(t)+\Gamma_{\lambda, \mathbf{k}}^{\mathrm{out}, c p+c c}(t)\right] \\
\mathcal{U}_{\mathbf{k}}(t) & =\mathcal{U}_{\mathbf{k}}^{c p}(t)+\sum_{\mathbf{k}^{\prime}}\left[\mathcal{U}_{\mathbf{k k}^{\prime}}^{v c}(t) p_{\mathbf{k}^{\prime}}(t)+\mathcal{U}_{\mathbf{k k}^{\prime}}^{c v}(t) p_{\mathbf{k}^{\prime}}^{*}(t)\right]
\end{aligned}
$$

Here, the nondiagonal dephasing contains a phonon- $\left(\mathcal{U}_{\mathrm{k}}^{c p}\right)$ and Coulomb-induced part (second term), which couples to all coherences in the Brillouin zone. The phonon off-diagonal contribution is given by

$$
\begin{array}{r}
\mathcal{U}_{\mathbf{k}}^{c p}(t)=\frac{\pi}{\hbar} \sum_{\lambda j \mathbf{q}}\left[\left|g_{\mathbf{k}+\mathbf{q}, \mathbf{q}}^{\lambda \lambda j}\right|^{2}\left[\left(1-\rho_{\mathbf{k}}^{\lambda}\right)\left(n_{\mathbf{q}}^{j}+1\right)+\rho_{\mathbf{k}}^{\lambda} n_{\mathbf{q}}^{j}\right] p_{\mathbf{k}+\mathbf{q}} \delta_{c p}^{+}\right. \\
\left.+\left|g_{\mathbf{k}, \mathbf{q}}^{\lambda \lambda j}\right|^{2}\left[\left(1-\rho_{\mathbf{k}}^{\lambda}\right) n_{\mathbf{q}}^{j}+\rho_{\mathbf{k}}^{\lambda}\left(n_{\mathbf{q}}^{j}+1\right)\right] p_{\mathbf{k}-\mathbf{q}} \delta_{c p}^{-}\right]
\end{array}
$$

with $\delta_{c p}^{ \pm}=\delta\left( \pm \varepsilon_{\mathbf{k} \pm \mathbf{q}}^{\lambda} \mp \varepsilon_{\mathbf{k}}^{\lambda}-\hbar \omega_{\mathbf{q}}^{j}\right)$ The contribution of the Coulomb interaction to the off-diagonal dephasing reads

$$
\begin{aligned}
& \mathcal{U}_{\mathbf{k k}^{\prime}}^{\lambda_{1} \lambda_{2}}=\frac{\pi}{\hbar} \sum_{\mathbf{a b}} \sum_{\lambda}\left[V_{\mathbf{k}^{\prime} \lambda_{2} \mathbf{k}_{b} \lambda_{b}}^{\mathbf{k} c \mathbf{k}_{c} \lambda_{c}} \tilde{V}_{\mathbf{k} v \mathbf{k}_{c} \lambda_{c}}^{\mathbf{k}^{\prime} \lambda_{1} \mathbf{k}_{b} \lambda_{b}}\left(\rho_{\mathbf{k}}^{\lambda}\left[1-\rho_{\mathbf{b}}\right] \rho_{\mathbf{c}}+\left[1-\rho_{\mathbf{k}}^{\lambda}\right] \rho_{\mathbf{b}}\left[1-\rho_{\mathbf{c}}\right]\right) \delta_{c c}^{+}\right. \\
& \left.-V_{\mathbf{k}_{b} \lambda_{b} \mathbf{k}_{c} \lambda_{c}}^{\mathbf{k} \mathbf{k}^{\prime} \lambda_{1}} \tilde{V}_{\mathbf{k} v \mathbf{k}^{\prime} \lambda_{2}}^{\mathbf{k}_{b} \lambda_{b} \mathbf{k}_{c} \lambda_{c}}\left(\left[1-\rho_{\mathbf{k}}^{\lambda}\right] \rho_{\mathbf{b}} \rho_{\mathbf{c}}+\rho_{\mathbf{k}}^{\lambda}\left[1-\rho_{\mathbf{b}}\right]\left[1-\rho_{\mathbf{c}}\right]\right) \delta_{c c}^{-}\right],
\end{aligned}
$$

where $\delta_{c c}^{ \pm}=\delta\left(\varepsilon_{\mathbf{k}}^{\lambda}+\sigma_{\lambda^{\prime}} \varepsilon_{\mathbf{k}^{\prime}}^{\lambda}-\varepsilon_{\mathbf{b}} \pm \varepsilon_{\mathbf{c}}\right)$. 


\subsection{Differential transmission spectroscopy}

The full set of the graphene Bloch equations provides a microscopic access to the ultrafast carrier dynamics. As shown in section 2.4, the description of the relaxation dynamics results is a time-, angle-, and momentum-resolved data of the microscopic polarization, the carrier and phonon occupation. While the measuring of the absorption or conductivity contains information about the polarization, it is difficult to directly measure the carrier or phonon distribution.

The most common technique to study the ultrafast carrier dynamics in graphene experimentally are pump-probe experiments, where differential transmission spectra (DTS) can be obtained: First a pump pulse $p$ at time zero excites the carrier system at the energy $\hbar \omega_{p}$ and creates a nonequilibrium. Then, after a certain time delay $\tau$, a test pulse $t$ scans the sample at the probe energy $\hbar \omega_{t}$, cf. Fig. 2.11. Typically, the signal is referred and normalized to the linear transmission of the probe pulse without previous pump pulse $T_{0}\left(\omega_{t}\right)$, resulting in the DTS formula:

$$
\frac{\Delta T}{T_{0}}=\frac{T\left(\omega_{p}, \omega_{t}, \tau\right)-T_{0}\left(\omega_{t}\right)}{T_{0}\left(\omega_{t}\right)} .
$$

The simplest experimental setup is the degenerated DTS with $\omega_{p}=\omega_{t}=\omega$. Using the relation $T=I / I_{0}$ with the intensity $I=I_{0} e^{-\alpha z}$ and assuming that the absorption $\alpha$ is relatively small (Taylor expansion), the DTS can be approximated in terms of absorbance including both the pump and the probe (test) pulse $\alpha^{(p, t)}(\omega, \tau)$ and only the test pulse $\alpha^{(t)}(\omega)$ 41, 62,:

$$
\frac{\Delta T}{T_{0}} \propto \alpha^{(t)}(\omega)-\alpha^{(p, t)}(\omega, \tau)
$$

Note, that this relation is only valid, if there is no interference between the pump and the test pulse. Taking into account only interband transitions, a delta-

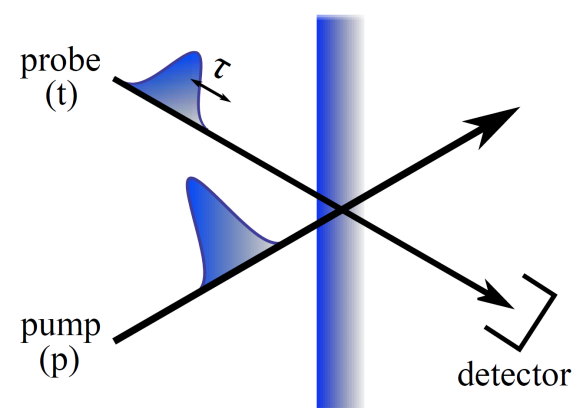

Figure 2.11.: Scheme of the differential transmission spectroscopy. A pump pulse excites the carrier system at a certain energy. The weak probe pulse scans the pump-induced transmission depending on the delay time and the probe frequency. The signal is referred and normalized to the transmission without previous pump pulse resulting in the differential transmission spectrum. Figure is taken from 41. shaped probe pulse, and an isotopic carrier occupation the DTS can be approximated by the pump-induced change in the carrier occupation [41]:

$$
\Delta T / T_{0}(\tau, \omega) \propto \Delta \rho_{k_{t}}^{(p)}(\tau)-\Delta \rho_{k_{t}}^{(p)}(-\infty)
$$

with $\Delta \rho(\tau)=\rho^{c}(\tau)-\rho^{v}(\tau)$. 


\section{Absorption spectra}

A better microscopic understanding of its optical and electronic properties is the key for exploiting the tremendous application potential of graphene. Recent experiments have revealed that the conductivity is characterized by a universal frequency-independent value in the infrared up to the visible spectral region and a pronounced excitonic peak. The optical features depend in detail on the number of graphene layers and the type of the stacking order $[9,10,20,63,65]$. Bilayer graphene consisting of weakly coupled two carbon monolayers exhibits as well as monolayer graphene remarkable optical and electronic properties [7-10|. In particular, its tunable band gap is promising for the design of future nanoelectronic devices $[11-\sqrt{16} \mid$. Similar to the monolayer, bilayer graphene shows a zero-gap band structure at the Dirac point. However, the crossing bands are not linear but parabolic describing electrons with a finite mass $9,13,14,42$ (cf. Fig. 2.4). The most stable stacking arrangement is known to be Bernal stacking (AB-stacking), where $\mathrm{A}$ atoms in one layer lie directly below or above the B atoms of the other layer [9], cp. Fig. 2.2. In addition to the stacking order, the Coulomb interaction influences the optical absorption giving rise to so-called saddle-point excitons in the ultraviolet region of the bilayer spectrum [66, 67].

Based on density functional theory calculations combined with the GW-BetheSalpeter equation, Yang and co-workers 8 have shown that the absorption spectrum of graphene is characterized by a pronounced excitonic transition near 4.5 $\mathrm{eV}$. They have also found a small low-energy peak that stems from interband transitions near the Dirac point. In the following studies [68,69|, Yang investigated the high-energy region involving transitions between $\sigma$ and $\pi$ bands as well as the influence of an applied electrical field on the low-energy spectrum within a free-particle picture. Furthermore, Chang presented an analytic model for the absorption spectrum of AB- and AA-stacked bilayer graphene, however, neglecting excitonic effects $|70|$.

In this chapter, the optical properties of mono- and bilayer graphene are investigated. In particular the absorption spectra are presented including the influence of the Coulomb-induced renormalization and excitons. Furthermore, the origin and the characteristics of the allowed single transitions located in the low-infrared up to the ultraviolet region are systematically studied in the absorption spectrum of bilayer graphene. 


\subsection{Linear optics}

This chapter focuses on the case of linear optics, where the electric field is considered to be small. As a result, the occupation in valence and conduction band is assumed to remain in thermodynamic equilibrium, i.e. the change in occupations is vanishingly small justifying the approximation of a full valence band $\left(\rho_{\mathbf{k}}^{v}=1\right)$, and an empty conduction band $\left(\rho_{\mathbf{k}}^{c}=0\right)$. Therefore, in the limiting case of linear optics, the dynamics of a system is fully determined by the microscopic polarization $p_{\mathbf{k}}(t)$.

Optical susceptibility The determination of the absorption coefficient $\alpha(\omega)$ requires the calculation of the optical susceptibility $\chi(\omega)$. The latter can be calculated either with the Fourier transform of the macroscopic polarization $P(\omega)$ or the current density $j(\omega)$. Within the $\mathbf{p} \cdot \mathbf{A}$ approach, the optical susceptibility can be expressed as a function of the current density $j(\omega)$ and the vector potential $A(\omega)$ via 71

$$
\chi(\omega)=\frac{P(\omega)}{\varepsilon_{0} E(\omega)}=\frac{j(\omega)}{\varepsilon_{0} \omega^{2} A(\omega)} .
$$

This equation results from the relations $\mathbf{j}=\partial_{t} \mathbf{P}, \mathbf{P}(\omega)=\frac{\mathbf{j}(\omega) i}{\omega}$ and $\mathbf{E}(\omega)=$ $-\partial_{t} \mathbf{A}(\omega)-\nabla \psi$, where the scalar potential $\phi$ vanishes within the radiation gauge for the field. The macroscopic current density is defined as 51

$$
\mathbf{j}(t)=\frac{e_{0}}{2 L^{2} m_{0}} \sum_{\lambda \lambda^{\prime}} \sum_{\mathbf{k k}^{\prime}} \sum_{s s^{\prime}}\left\langle\Psi_{\mathbf{k}}^{\lambda}(\mathbf{r})\left|\left(\mathbf{p}-e_{0} \mathbf{A}(\mathbf{r}, t)\right)\right| \Psi_{\mathbf{k}^{\prime}}^{\lambda^{\prime}}(\mathbf{r})\right\rangle\left\langle a_{\mathbf{k}}^{\lambda \dagger} a_{\mathbf{k}^{\prime}}^{\lambda^{\prime}}\right\rangle+\text { c.c. }
$$

where the double spin sum yields a factor $\sigma_{s}=2$, since no interactions changing the spin are considered. After applying the dipole approximation with $\mathbf{A}(\mathbf{r}, t) \approx \mathbf{A}(t)$ and introducing the optical matrix element $\mathbf{M}_{\mathbf{k}}^{\lambda \lambda^{\prime}}$, the current reads

$$
\mathbf{j}(t)=\frac{2 e_{0} \hbar}{m_{0} L^{2}} \sum_{\mathbf{k}}\left[2 \mathbf{M}_{\mathbf{k}}^{v c} \operatorname{Im}\left[p_{\mathbf{k}}(t)\right]-i \sum_{\lambda} \mathbf{M}_{\mathbf{k}}^{\lambda \lambda} \rho_{\mathbf{k}}^{\lambda}(t)\right]+\frac{e_{0}^{2}}{m_{0}} \mathbf{A}(t) n
$$

with the electronic density $n=\left(2 / L^{2}\right) \sum_{\lambda \mathbf{k}} \rho_{\mathbf{k}}^{\lambda}$. The current contains an intraband (fist term) and an interband (second term) contribution and a term directly driven by the vector potential (last term). In equilibrium, the carrier distribution $\rho_{\mathbf{k}}^{\lambda}$ is symmetric with respect to the Dirac point. Since the optical intraband matrix element is antisymmetric, the intraband current vanishes. The last term including the electronic density corresponds to a Drude-like response, which does not have an 
imaginary part and therefore does not directly contribute to the optical absorption. For bilayer graphene, the current density yields within the linear limit 46

$$
\begin{aligned}
\mathbf{j}(t)=\frac{4 e_{0} \hbar}{L^{2} m_{0}} \sum_{\mathbf{k}}\left[\mathbf{M}_{\mathbf{k}}^{v_{1} c_{1}} \operatorname{Im}\left[p_{\mathbf{k}}^{v_{1} c_{1}}(t)\right]+\mathbf{M}_{\mathbf{k}}^{v_{2} c_{2}} \operatorname{Im}\left[p_{\mathbf{k}}^{v_{2} c_{2}}(t)\right]\right. \\
\left.\quad-i\left(\mathbf{M}_{\mathbf{k}}^{v_{1} c_{2}} \operatorname{Re}\left[p_{\mathbf{k}}^{v_{1} c_{2}}(t)\right]+\mathbf{M}_{\mathbf{k}}^{v_{2} c_{1}} \operatorname{Re}\left[p_{\mathbf{k}}^{v_{2} c_{1}}(t)\right]\right)\right]-\frac{e_{0}^{2}}{m_{0}} \mathbf{A}(t) n .
\end{aligned}
$$

The cross transitions $v_{1} \rightarrow c_{2}$ and $v_{2} \rightarrow c_{1}$ characterized by $\left|\mathbf{M}_{\mathbf{k}}^{v_{1} c_{2}}\right|^{2}=\left|\mathbf{M}_{\mathbf{k}}^{v_{2} c_{1}}\right|^{2}$ and the same energy gaps (cp. Fig. 2.4) are expected to equally contribute to the optical spectrum. In contrast, the transitions $v_{1} \rightarrow c_{1}$ and $v_{2} \rightarrow c_{2}$ should lead to distinct features in the absorption spectrum, since they are characterized by different bandgaps.

Absorption To obtain the absorption coefficient for graphene as a single layer of atoms, the contribution of the reflected and transmitted electrical is taken into account $17,41,72,73$. The resulting absorbance reads

$$
\alpha(\omega)=\frac{\omega}{c_{0} n_{B}} \frac{\operatorname{Im}[\chi(\omega)]}{\left|1-i \frac{\omega}{2 c_{0} n(\omega) \chi(\omega)}\right|^{2}}
$$

with the light velocity $c_{0}$. The absorbance of graphene can be interpreted as absorption per graphene layer [8,66]. For free-suspended graphene, the background refraction index $n(\omega)$ can be set to one. The renormalization in the denominator stems from the radiative damping in the graphene sample [41.

Screening It is important to consider taking into account that the screening depends on the single positions of the particles, which is in detail described in Ref. $\mid 74$. . As a result, the screening is given by

$$
\varepsilon_{\mathbf{G}_{1}, \mathbf{G}_{2}}(\mathbf{q})=\delta_{\mathbf{G}_{1}, \mathbf{G}_{2}}-\sum_{12} \Pi^{12} \delta_{q,\left|\mathbf{k}_{2}-\mathbf{k}_{1}\right|} L_{21}^{12}\left(-\mathbf{G}_{1},-\mathbf{G}_{2}\right) V_{\mathbf{q}}\left(q+\mathbf{G}_{2}\right)
$$

with an arbitrary reciprocal lattice vector $\mathbf{G}_{i}$ and the function $L_{34}^{12}\left(\mathbf{G}_{1}, \mathbf{G}_{2}\right)=$

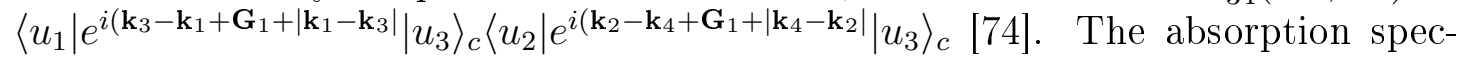
trum of graphene including the derived dielectric function $\varepsilon_{\mathbf{G}_{1}, \mathbf{G}_{2}}(\mathbf{q})$ will be presented in the next section. 


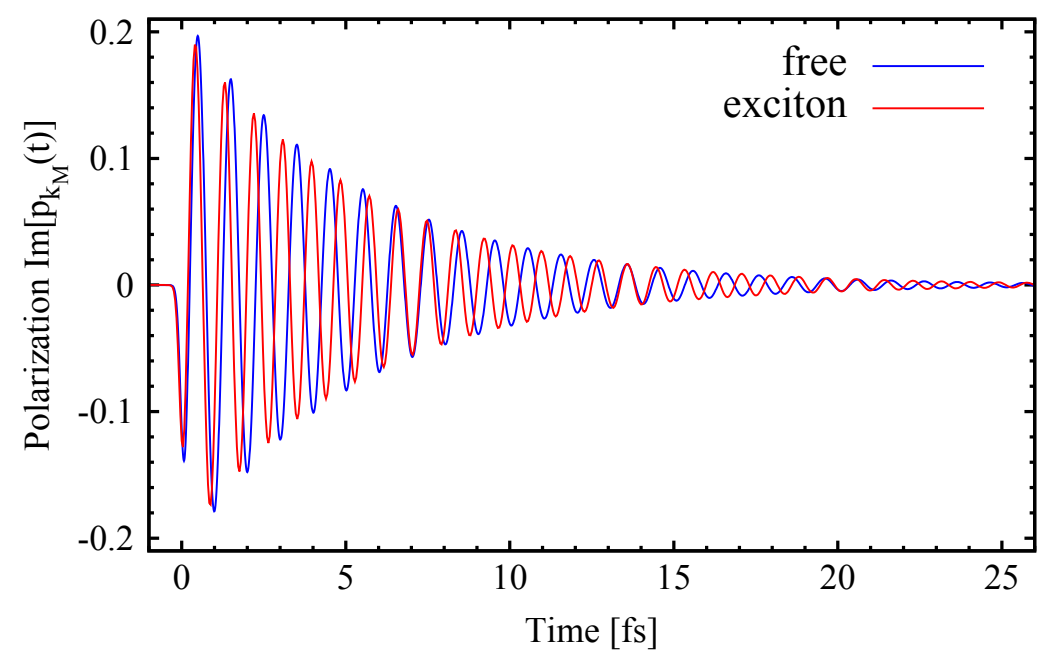

Figure 3.1.: Temporal evolution of the imaginary part of the polarization $p_{k_{M}}(t)$ at the $M$ point. The different frequency and shape of the excitonic polarization in comparison with the free-particle part demonstrates the importance of the Coulomb interaction.

\subsection{Excitonic absorption spectra}

\subsubsection{Graphene}

To obtain the absorption coefficient $\alpha(\omega)$, the numerical evaluation of the Bloch equation for the microscopic polarization $p_{\mathbf{k}}(t)$ is needed. Taking into account only the Hartree-Fock contribution, the Bloch equation for the polarization reads within the linear limit

$$
\begin{aligned}
\dot{p}_{\mathbf{k}}(t)= & {\left[i \omega_{\mathbf{k}}^{v c}+\frac{i}{\hbar} \sum_{\mathbf{k}^{\prime}} V_{\mathrm{ren}^{\mathbf{k}}}^{\mathbf{k} \mathbf{k}^{\prime}}-\gamma\right] p_{\mathbf{k}}(t)+i \Omega_{\mathbf{k}}^{v c}(t) } \\
& +\frac{i}{\hbar} \sum_{\mathbf{k}^{\prime}}\left(V_{\mathbf{k} v, \mathbf{k}^{\prime} c}^{\mathbf{k}^{\prime} v, \mathbf{k} c} p_{\mathbf{k}^{\prime}}(t)+V_{\mathbf{k} v, \mathbf{k}^{\prime} v}^{\mathbf{k}^{\prime} c, \mathbf{k}} p_{\mathbf{k}^{\prime}}^{*}(t)\right) .
\end{aligned}
$$

with the phenomenological damping constant $\gamma=(0.12 / \hbar) \mathrm{eV}$, which describes the carrier-phonon scattering [41]. Figure 3.1 illustrates the temporal evolution of the imaginary part of the polarization $p_{\mathbf{k}}(t)$ at the $M$ point in the free-particle and excitonic picture. The frequency and the damping of the oscillation reflect the position and the width of the absorption peak in the spectrum. The different frequency and shape of the excitonic polarization in comparison with the free-particle part illustrates the importance of the Coulomb interaction. The change of the frequency results from the renormalization term $\frac{i}{\hbar} \sum_{\mathbf{k}^{\prime}} V_{\text {ren }}^{\mathbf{k k}^{\prime}}$, whereas the different 


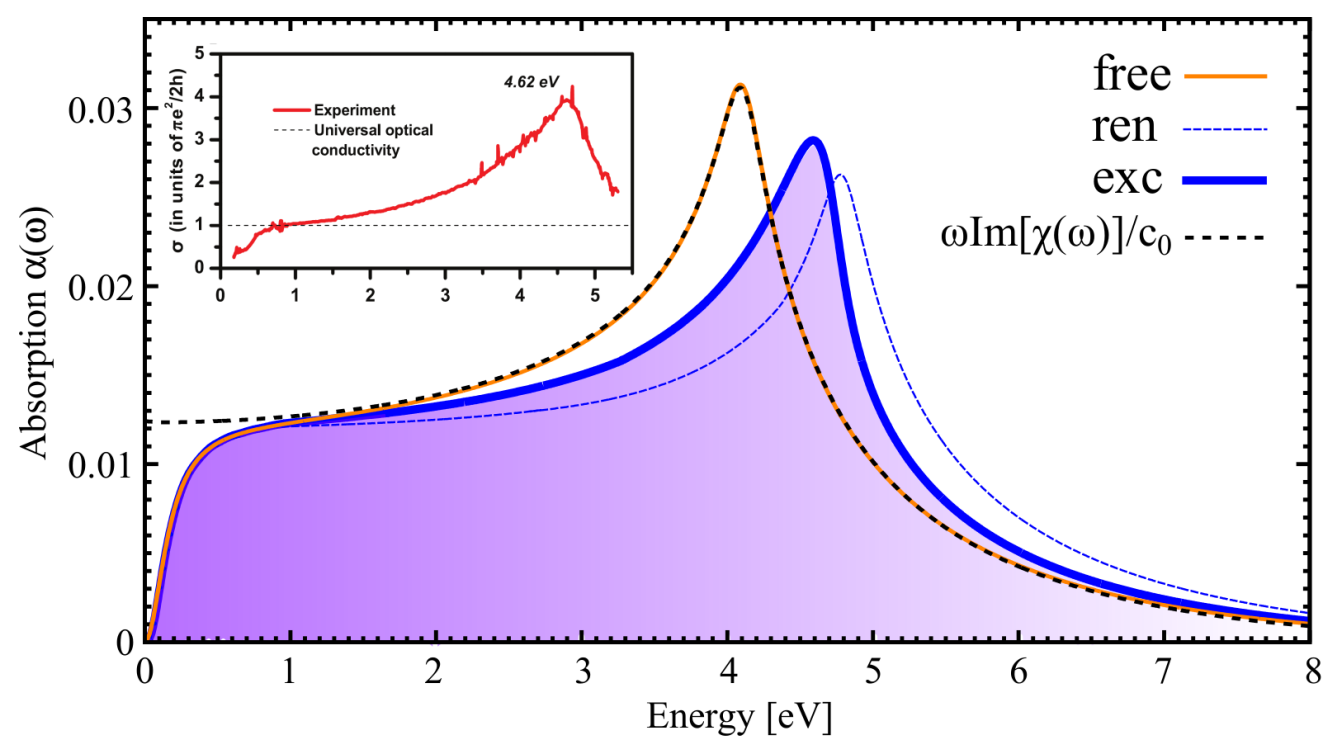

Figure 3.2.: Free-particle, renormalized, and excitonic absorption spectrum of graphene illustrating the importance of the repulsive carrier-carrier and attractive carrier-hole part of the Coulomb interaction. The spectrum is characterized by a almost constant absorbance between 1 and $2 \mathrm{eV}$ and a drop-off in the signal at energies below $0.5 \mathrm{eV}$. Without the denominator in Eq. 3.5 (black dashed line), the absorbance is almonst constant between 0 and $2 \mathrm{eV}$. The inset illustrates the experimental obtained conductivity of graphene. Experimental data is taken from 66 .

shape in the oscillation is described by the last term proportional to $p_{\mathbf{k}^{\prime}}(t)$ of Eq. 3.7. Inserting the numerical solution of the linear limit of the Bloch equation in the current density (Eq. 3.3), the absorption can be calculated via Eq. 3.5.

Figure 3.2 shows the free-particle, Coulomb-renormalized and excitonic absorption spectrum of graphene. The free-particle absorbance exhibits at approximately $4 \mathrm{eV}$ a well pronounced peak corresponding to the free-particle bandgap at the $M$ point. The position of the $M$ point and therefore of the pronounced peak is determined by the tight-binding parameter $\gamma_{0}$. The observed peak exhibits a large width of approximately $2 \mathrm{eV}$ reflecting the broad density of states at the saddlepoint. The peak shape and its intensity is influenced by the interband optical matrix element $M_{\mathbf{k}}^{v c}$, which shows a maximal optical activity at the $M$ point and suppressing it at the $\Gamma$ point. In good agreement with experimental results [66], the absorbance is nearly constant in the near-infrared region between 1 and $2 \mathrm{eV}$. This can be traced back to the interplay between the density of states, which scales linearly with the frequency $\omega$, and the dependence of the absorbance on 
$1 / \omega$. Within the applied tight-binding method including effective carbon atom orbital functions, the constant absorbance value depends on the effective atomic number $\left.Z_{\text {eff }} \mid 75\right]$, which determines the optical matrix element, cp. appendix A.1. Taking the denominator of Eq. 3.5 into account, the absorbance is considerably reduced for photon energies below $0.5 \mathrm{eV}$. This observation is due to the radiation coupling, which leads to a renormalization of the absorption coefficient 41.

In distinction from the free-particle absorption spectrum, the experiment shows an asymmetric peak shape at a different energetic position. To directly illustrate the influence of the Coulomb interaction on the absorption spectrum, the contribution of the repulsive electron-electron and the attractive electron-hole coupling is separately investigated, cp. Fig. 3.2. The Coulomb interaction is known to be strong in low-dimensional carbon nanostructures $[76,77 \mid$. Even in metallic carbon nanotubes [58,59,78,79] and gapless graphene [8, 17, 66], the optical oscillator strength is transferred to a large extent to excitons. This can be ascribed to the much weaker many-particle screening compared to conventional metallic structures. In graphene the screening of the Coulomb potential is very efficient due to the crossing bands at the Dirac point, and needs to be explicitly taken into account $58,59,78,79]$. The momentum-dependent dielectric function $\varepsilon(\mathbf{q})$ has been derived consistently within the density matrix formalism by taking into account higher-order contributions within the correlation expansion, see section 2.3.2. The calculations reveal an efficient screening in graphene for small momentum transfers. As a result, the many-particle screening completely cancels any Coulomb contribution in the absorption spectrum at low energies, the free-particle spectrum is identical to the renormalized as well as excitonic spectrum for photon energies up to $1 \mathrm{eV}$. Note, that this can be only observed, if the full spatial dependence of the screened Coulomb interaction is taken into account, see Eq. 3.6.

First, the repulsive part of the electron-electron interaction will be discussed, cp. the dashed line in Fig 3.2. A pronounced Coulomb-induced blue-shift of the saddle-point transition by approximately $4.7 \mathrm{eV}$ is observed. The repulsive part of the Coulomb interaction $V_{\text {ren }}\left(\mathbf{k}, \mathbf{k}^{\prime}\right)$ in Eq. (3.7) gives rise to a significant renormalization of the electronic free-particle band structure of graphene. It leads to a larger band gap at the $M$ point, which explains the blue-shift.

Including the excitonic part of the Coulomb interaction (last term of Eq. 3.7) changes the position and the shape of the absorption peak accounting for the formation of of electron-hole pairs at the $M$ point (saddle-point excitons), cp. the thick line in Fig. 3.2. The observed red-shift of the Coulomb-renormalized spectrum of approximately $200 \mathrm{meV}$ represent the excitonic binding energy. Similarly to metallic carbon nanotubes, higher excitonic transitions cannot be observed in the spectrum of graphene. The change in the peak shape results from the redistribution of the momentum and the transfer of the oscillator strength into the excitonic transition. In agreement with experiments [66, 80], an asymmetric peak 


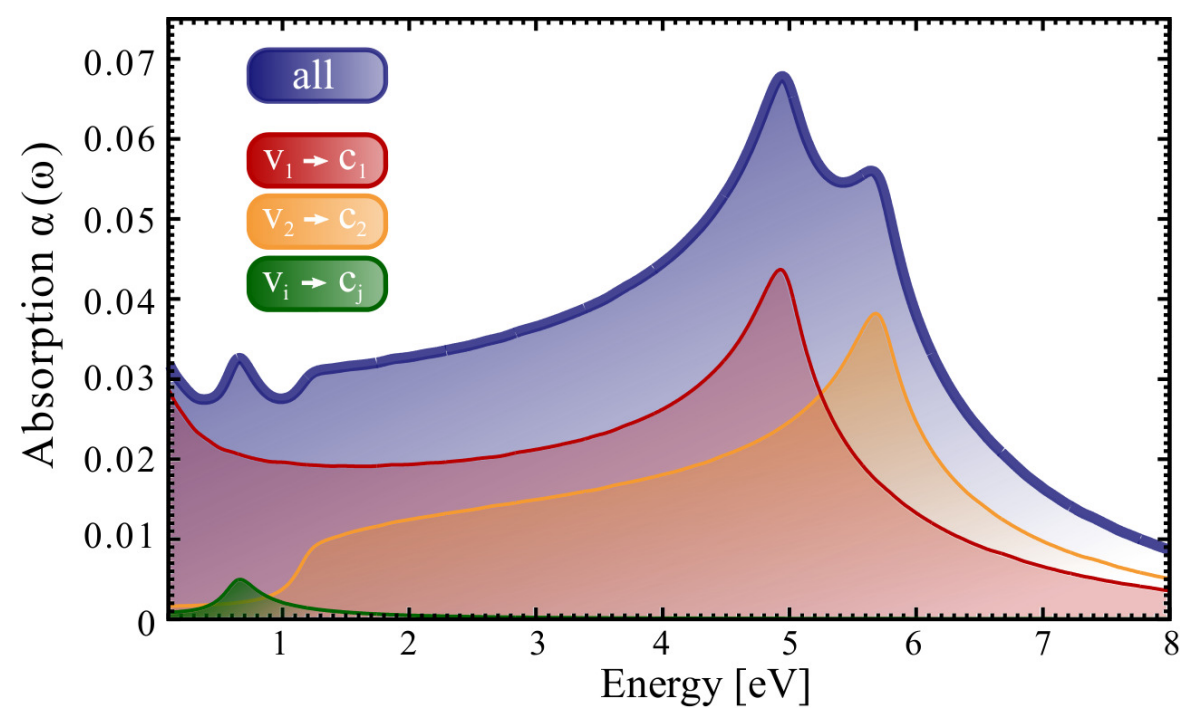

Figure 3.3.: Excitonic absorption spectrum of bilayer graphene over a large spectral range, where the contribution of single optical transitions is shown. In the ultraviolet region, the spectrum exhibits two energetically close absorption peaks resulting from interband transitions $v_{i} \rightarrow c_{i}$ with $i=(1,2)$ at the $M$ point. At low energies, the spectrum is characterized by a small peak, which is induced by cross transitions $v_{i} \rightarrow c_{j}$ with $i \neq j$ at the Dirac point. Figure is adopted from [46].

is observed, cp. Fig. 3.2, where the asymmetry can be ascribed to an overlap of the excitonic transition with the contribution of the free-continuum.

\subsubsection{Bilayer graphene}

In bilayer graphene, the temporal evolution of the polarization within the HartreeFock approximation is given by

$$
\begin{aligned}
\dot{p}_{\mathbf{k}}^{\lambda_{i} \lambda_{j}^{\prime}}(t)= & {\left[i \omega_{\mathbf{k}}^{\lambda_{i} \lambda_{j}^{\prime}}+\frac{i}{\hbar} \sum_{\mathbf{k}^{\prime}} V_{\mathrm{ren}}^{\lambda_{i} \lambda_{j}^{\prime}}-\gamma\right] p_{\mathbf{k}}(t)+i \Omega_{\mathbf{k}}^{\lambda_{i} \lambda_{j}^{\prime}}(t) } \\
& +\frac{i}{\hbar} \sum_{\mathbf{k}^{\prime}}\left(V_{\mathbf{k} \lambda_{i}, \mathbf{k}^{\prime} \lambda_{j}^{\prime}}^{\mathbf{k}^{\prime} \lambda_{i}, \mathbf{k} \lambda_{j}^{\prime}} p_{\mathbf{k}^{\prime}}^{\lambda_{i} \lambda_{j}^{\prime}}(t)+V_{\mathbf{k} \lambda_{i}, \mathbf{k}^{\prime} \lambda_{i}}^{\mathbf{k}^{\prime} \lambda_{j}^{\prime}, \mathbf{k} \lambda_{\mathbf{k}^{\prime}}^{\prime}} p^{\lambda_{i} \lambda_{j}^{\prime} *}(t)\right) .
\end{aligned}
$$

Here, different transitions $\lambda_{i} \rightarrow \lambda_{j}^{\prime}$ have to be considered to obtain the full absorption spectrum. Figure 3.3 shows the excitonic absorption spectrum of bilayer graphene over a large spectral range. To obtain profound insights into the optical properties of bilayer graphene and the underlying elementary processes, the contributions of single optical transitions is presented, cp. Eq. 3.8. The calculations 


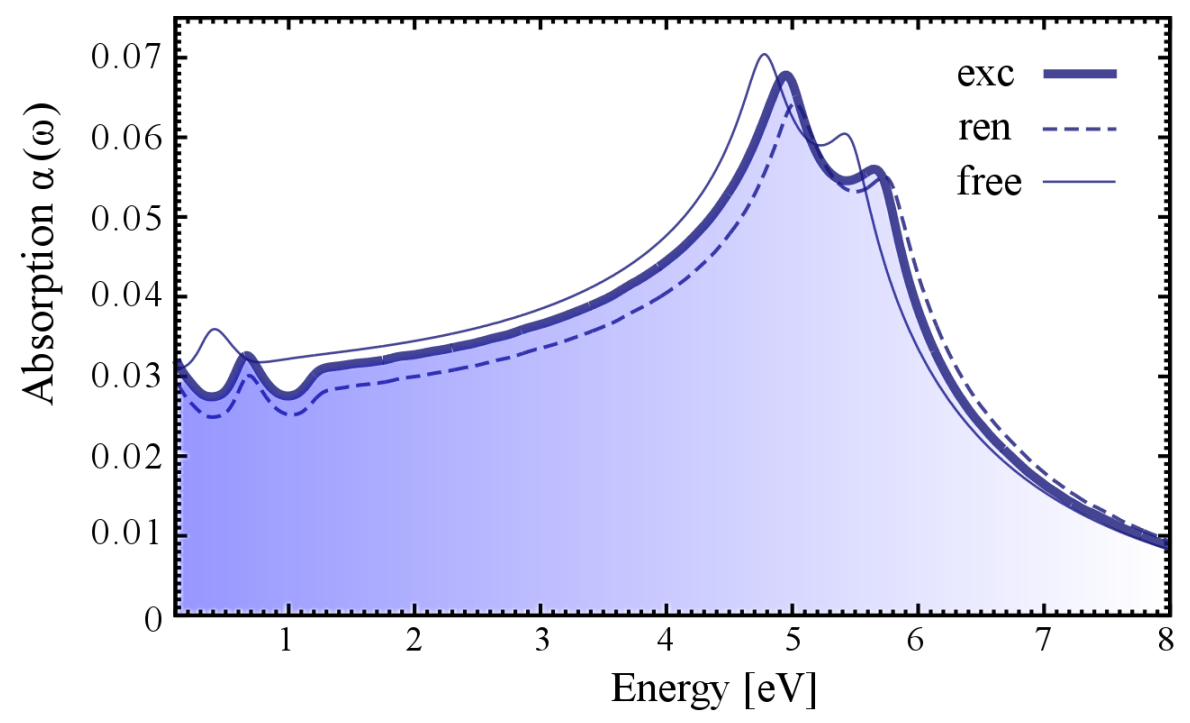

Figure 3.4.: Free-particle, Coulomb-renormalized, and excitonic absorption spectrum of bilayer graphene illustrating the importance of the repulsive carrier-carrier and attractive carrier-hole part of the Coulomb interaction. Figure is adopted from $[46$.

predict the appearance of three pronounced absorption peaks at $0.7 \mathrm{eV}, 4.9 \mathrm{eV}$, and $5.7 \mathrm{eV}$, respectively. The striking difference to the spectrum of monolayer graphene lies in the appearance of the low-intensity peak at $0.7 \mathrm{eV}$. It can be clearly ascribed to the cross transitions $v_{i} \rightarrow c_{j}$ at the Dirac point, cp. Fig. 2.4. The corresponding energy gap $\hbar \omega_{K}^{v_{i} c_{j}}$ exactly reflects the spectral position of the predicted peak and the optical matrix element $\left|\mathbf{M}_{\mathbf{k}}^{v_{i} c_{j}}\right|^{2}$ determining its oscillator strength exhibits a maximum at the Dirac point, as illustrated in Fig. 2.6. This low-intensity peak overlaps the featureless frequency-independent absorption region and has already been experimentally observed [63|. In the ultra-violet region of the spectrum, two pronounced absorption peaks appear resulting from the transitions $v_{1} \rightarrow c_{1}$ and $v_{2} \rightarrow c_{2}$, respectively. Their spectral position reflects the corresponding energy gaps $\hbar \omega_{M}^{v_{i} c_{i}}$ at the $M$ point, cp. Fig. 2.4. They are characterized by the same oscillator strength determined by the optical matrix element $\left|\mathbf{M}_{\mathbf{k}}^{v_{i} c_{i}}\right|^{2}$, which exhibits a clear maximum at the saddle point, cp. Fig. 4.3. The energetically lower transition $v_{1} \rightarrow c_{1}$ is higher in intensity due to the larger overlap with the lowenergy shoulder of the $v_{2} \rightarrow c_{2}$ transition in addition to the $1 / \omega$ dependence of the absorption coefficient, cp. Eq. 3.5. Furthermore, the contribution of the transition $v_{2} \rightarrow c_{2}$ vanishes in the near-infrared region reflecting the small bandgap of $2\left|\gamma_{1}\right|$ between the two involved bands, cp. Fig. 2.4. There is no pronounced absorption at this point despite the enhanced density of states, since the corre- 
sponding optical matrix elements vanish at the Dirac point, as shown in Fig. 2.6. The peak width is determined by the dephasing of the microscopic polarization resulting from Coulomb- and phonon-induced many-particle correlations beyond the Hartree-Fock level. In this thesis, the dephasing is considered within a typical phenomenological constant $\gamma=(0,12 / \hbar) \mathrm{eV}$. In most experiments, only one spectrally broad peak has been observed in the ultra-violet region suggesting a larger broadening in the investigated samples of bilayer graphene 66, 80.

To illustrate the influence of the Coulomb interaction on the absorption spectrum of bilayer graphene, the contribution of the repulsive electron-electron and the attractive electron-hole coupling is separately investigated, cp. Fig. 3.4. The electron-electron coupling leads to a considerable overall renormalization of the band structure. The additional term $V_{\mathrm{ren}}^{\lambda_{i} \lambda_{j}}$ in Eq. 2.58 enlarges the band gaps at the saddle point and the Dirac point giving rise to a spectrum blue-shifted by approximately $0.3 \mathrm{eV}$ compared to the free-particle case, cp. the thin solid and the dashed line in Fig. 3.4. Similar to monolayer graphene, the strong Coulomb interaction gives rise to the formation of electron-hole pairs at the $M$ point [8, 17, 66, 80]. As a result, the spectrum at the saddle-point is considerably rearranged, where a clear red-shift of approximately $100 \mathrm{meV}$ reflecting the excitonic binding energy for the transitions $v_{1} \rightarrow c_{1}$ and $v_{2} \rightarrow c_{2}$ is observed. This binding energy is smaller compared to graphene $8,17,66$ due to the increased many-particle screening in the presence of two carbon layers. The microscopically obtained dielectric function $\varepsilon(\mathbf{q})$ reaches values of above 13 [see Fig. 2.8] for a small momentum transfer $q$, which is clearly larger than in graphene 41]. As a result, the Coulomb matrix element is weak close to the Dirac point no exciton-induced red-shift of the lowenergy peak is observed, cp. the thick solid and dashed line in Fig. 3.4. 


\section{Impact of intraband absorption in graphene}

The ultrafast carrier relaxation dynamics in optically excited graphene has been intensively studied [18 27, 81]. Typically, the carrier relaxation has been accessed via high-resolution pump-probe experiments $22,23,25,81,82$. Common to all studies is a bi-exponential decay of the pump-induced differential transmission (DT) spectrum. The fast decay component in the range of few tens of femtoseconds is assigned to an ultrafast Coulomb-dominated carrier redistribution towards a hot FermiDirac distribution, whereas the slower decay component in the range of a picosecond reflects the equilibration between the electron and the phonon system 22]. However, while some of the studies report on a purely positive transient DT spectrum [18, 20, 83, others exhibit a zero-crossing after the initial decay $19,22,23,81,84,86$. The second decay component characterizes the recovering of the negative transient DT signal. There is a number of possible underlying mechanisms for the DT-zero crossing 19, 22, 23, 26, 41, 81, 84, 86. For some of the experiments, the negative DT can be clearly traced back to the predominant intraband absorption. This is the case, when the photon energy is smaller than twice the value of the Fermi energy [23, 81, 85. However, for all other cases, where negative DT components occur for photon energies much larger

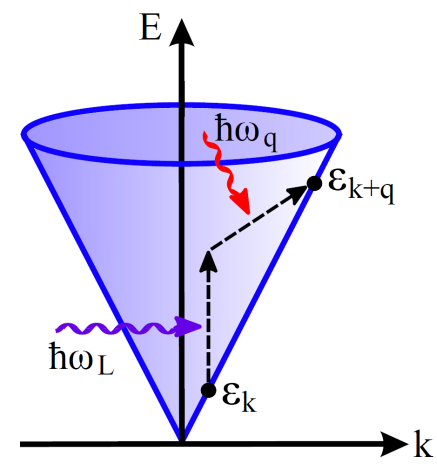

Figure 4.1.: Illustration of the phonon-assisted intraband absorption within the linear band structure of graphene. Applying a pulse with the energy $\hbar \omega_{L}$ lifts electrons within the (conduction) band into an energetically higher electronic state. To conserve energy and momentum, this intraband absorption is assisted by an interaction with a phonon characterized by the energy $\hbar \omega_{\mathbf{q}}$ and the momentum q. Figure is taken from [56]. than the Fermi energy, the underlying mechanism has been controversially discussed in literature. In particular, pump-induced bandstructure renormalization for transient $[22$ or in the long time limit $[87 \mid$ at the $M$-point as well as intraband absorption 24] have been suggested as explanations for the observed neg- 
ative DT signals. In this chapter a microscopic explanation for the occurrence of transient negative differential transmission in graphene is presented, where the detailed interplay of intra- and interband absorption processes on the transient DT in graphene is investigated. The absorptive electronic intraband transitions are assisted by emission or absorption of a phonon with an adequate momentum necessary to fulfil the momentum conservation, cf. Fig,4.1. Intraband transitions are shown to lead to an enhanced absorption giving rise to the experimentally observed zero-crossing from positive to negative DT signals.

\subsection{Phonon-assisted intraband absorption processes}

To determine the interplay of intra- and interband absorption processes on the transient DT in graphene, the equations of motion for the carrier occupation probability $\rho_{\mathbf{k}}^{\lambda}$ and the microscopic polarization $p_{\mathbf{k}}$ have to be derived. Here, the main focus lies on the carrier occupation, since the intraband absorption processes directly result from the phonon-assisted quantities. Applying the Heisenberg equation of motion the occupation reads

$$
\left.\dot{\rho}_{\mathbf{k}}^{\lambda}\right|_{H_{c, p}}=\frac{1}{i \hbar} \sum_{\lambda^{\prime}} \sum_{j \mathbf{q}}\left(g_{\mathbf{k}, \mathbf{q}}^{\lambda \lambda^{\prime} j} S_{\mathbf{k}, \mathbf{q}}^{\lambda \lambda^{\prime} j}-g_{\mathbf{k}+\mathbf{q}, \mathbf{q}}^{\lambda^{\prime} \lambda j} S_{\mathbf{k}+\mathbf{q}, \mathbf{q}}^{\lambda^{\prime} \lambda j}-g_{\mathbf{k}, \mathbf{q}}^{\lambda \lambda^{\prime} j *} T_{\mathbf{k}, \mathbf{q}}^{\lambda^{\prime} \lambda j}+g_{\mathbf{k}+\mathbf{q}, \mathbf{q}}^{\lambda^{\prime} \lambda j *} T_{\mathbf{k}+\mathbf{q}, \mathbf{q}}^{\lambda \lambda^{\prime} j}\right),
$$

where $S_{\mathbf{k}, \mathbf{q}}^{\lambda \lambda^{\prime} j}=\left\langle a_{\mathbf{k}}^{\lambda \dagger} a_{\mathbf{k}-\mathbf{q}}^{\lambda^{\prime}} b_{\mathbf{q}}^{j}\right\rangle$ and $T_{\mathbf{k}, \mathbf{q}}^{\lambda \lambda^{\prime} j}=\left\langle a_{\mathbf{k}-\mathbf{q}}^{\lambda \dagger} a_{\mathbf{k}}^{\lambda^{\prime}} b_{\mathbf{q}}^{j \dagger}\right\rangle$ are phonon-assisted electron densities and transitions [88]. To obtain a closed system of differential equations, additional equations for the phonon-assisted quantities must be derived [88, 89]. Exemplary, the equation of the temporal evolution of $S_{\mathbf{k}, \mathbf{q}}^{\lambda_{1} \lambda_{2} j}$ is given by

$$
\begin{aligned}
\dot{S}_{\mathbf{k}, \mathbf{q}}^{\lambda_{1} \lambda_{2} j}= & \frac{i}{\hbar} \Delta \varepsilon_{\mathbf{k}, \mathbf{q}}^{\lambda_{1} \lambda_{2}} S_{\mathbf{k}, \mathbf{q}}^{\lambda_{1} \lambda_{2} j}+\frac{i}{\hbar} \sum_{\lambda_{3} \lambda_{4}} g_{\mathbf{k}, \mathbf{q}}^{\lambda_{3} \lambda_{4} j *} Q_{\mathbf{k}, \mathbf{q}}^{\lambda_{1} \lambda_{3} \lambda_{4} \lambda_{2} j} \\
& +\frac{\hbar e_{0}}{m_{0}} \sum_{\lambda^{\prime}}\left[S_{\mathbf{k}, \mathbf{q}}^{\lambda_{1} \lambda^{\prime} j} \mathbf{M}_{\mathbf{k}-\mathbf{q}}^{\lambda_{2} \lambda^{\prime}}-S_{\mathbf{k}, \mathbf{q}}^{\lambda^{\prime} \lambda_{2} j} \mathbf{M}_{\mathbf{k}}^{\lambda^{\prime} \lambda_{1}}\right] \cdot \mathbf{A}(t)
\end{aligned}
$$

with the energy $\Delta \varepsilon_{\mathbf{k}, \mathbf{q}}^{\lambda_{1} \lambda_{2}}=\varepsilon_{\mathbf{k}}^{\lambda_{1}}-\varepsilon_{\mathbf{k}-\mathbf{q}}^{\lambda_{2}}-\hbar \omega_{\mathbf{q}}^{j}$ and the scattering kernel $Q_{\mathbf{k}, \mathbf{q}}^{\lambda_{1} \lambda_{2} \lambda_{3} \lambda_{4} j}=$ $\left(\delta_{\lambda_{1} \lambda_{2}}-\sigma_{\mathbf{k}}^{\lambda_{1} \lambda_{2}}\right) \sigma_{\mathbf{k}-\mathbf{q}}^{\lambda_{3} \lambda_{4}} n_{\mathbf{q}}^{j}-\sigma_{\mathbf{k}}^{\lambda_{1} \lambda_{2}}\left(\delta_{\lambda_{3} \lambda_{4}}-\sigma_{\mathbf{k}-\mathbf{q}}^{\lambda_{3} \lambda_{4}}\right)\left(n_{\mathbf{q}}^{j}+1\right)$, where $\sigma_{\mathbf{k}}^{\lambda \lambda^{\prime}}=\left\langle a_{\mathbf{k}}^{\lambda^{\dagger \dagger}} a_{\mathbf{k}}^{\lambda^{\prime}}\right\rangle$. The corresponding equation for $T_{\mathbf{k}, \mathbf{q}}^{\lambda \lambda^{\prime} j}$ can be derived in analogy |17, 41.

The last term in Eq. 4.2 is of crucial interest, since it describes phonon-assisted optical transitions. They are driven by both carrier-light interaction $\left(\mathbf{M}_{\mathbf{k}}\right)$ and implicitly also by carrier-phonon matrix elements appearing in the dynamics of the 
phonon-assisted quantity $S_{\mathbf{k}, \mathbf{q}}^{\lambda_{1} \lambda_{2} j}$. The main goal is the description of the phononassisted intraband absorption processes affecting the weak probe pulse applied after a much stronger pump pulse that creates a non-equilibrium carrier distribution. Since the experimentally observed negative transient DT spectrum occurs clearly after the application of the optical excitation, the interaction of the test pulse with the carrier occupation resulting from the pump-induced optical dynamics is studied 22,26$]$. To be able to solve Eq. (4.2) analytically, a perturbative approach is used assuming that 90

$$
S_{\mathbf{k}, \mathbf{q}}^{\lambda_{1} \lambda_{2} j}=S_{\mathbf{k}, \mathbf{q}(0)}^{\lambda_{1} \lambda_{2} j}+\epsilon S_{\mathbf{k}, \mathbf{q}(1)}^{\lambda_{1} \lambda_{2} j},
$$

where $\epsilon$ represents a small perturbation corresponding to the amplitude of the vector potential $\mathbf{A}_{\text {test }}(t)=\mathbf{A}_{0} e^{-t^{2} /\left(2 \sigma^{2}\right)} \cos \left(\omega_{L} t\right)$ describing the probe pulse with $\sigma$ as the pulse duration. Within the well tested Markov approximation, [17] the zeroth and the first order of $S_{\mathbf{k}, \mathbf{q}}^{\lambda_{1} \lambda_{2} j}$ are obtained analytically reading [56]

$$
\begin{aligned}
& S_{\mathbf{k}, \mathbf{q}(0)}^{\lambda_{1} \lambda_{2} j}=i \pi \sum_{\lambda_{3} \lambda_{4}} g_{\mathbf{k}, \mathbf{q}}^{\lambda_{3} \lambda_{4} j *} Q_{\mathbf{k}, \mathbf{q}}^{\lambda_{1} \lambda_{3} \lambda_{4} \lambda_{2} j} \delta\left(\Delta \varepsilon_{\mathbf{k}, \mathbf{q}}^{\lambda_{3} \lambda_{4}}\right), \\
& S_{\mathbf{k}, \mathbf{q}(1)}^{\lambda_{1} \lambda_{2} j}=i \pi \sum_{\lambda_{3} \lambda_{4}} g_{\mathbf{k}, \mathbf{q}}^{\lambda_{3} \lambda_{4} j *} Q_{\mathbf{k}, \mathbf{q}}^{\lambda_{1} \lambda_{3} \lambda_{4} \lambda_{2} j} \frac{\frac{i e_{0}}{m_{0}} \Delta M_{\mathbf{k}, \mathbf{q}}^{\lambda \lambda^{\prime}} e^{-\frac{t^{2}}{2 \sigma^{2}}}}{2 \Delta \varepsilon_{\mathbf{k}, \mathbf{q}}^{\lambda_{1} \lambda_{2}}} \\
& \quad \times\left[e^{-i \omega_{L} t} \delta\left(\Delta \varepsilon_{\mathbf{k}, \mathbf{q}}^{\lambda_{3} \lambda_{4}}+\hbar \omega_{L}\right)+e^{i \omega_{L} t} \delta\left(\Delta \varepsilon_{\mathbf{k}, \mathbf{q}}^{\lambda_{3} \lambda_{4}}-\hbar \omega_{L}\right)\right]
\end{aligned}
$$

with $\Delta M_{\mathbf{k}, \mathbf{q}}^{\lambda \lambda^{\prime}}=\left(\mathbf{M}_{\mathbf{k}-\mathbf{q}}^{\lambda^{\prime} \lambda^{\prime}}-\mathbf{M}_{\mathbf{k}}^{\lambda \lambda}\right) \cdot \hat{\mathbf{e}}_{A}$. All terms proportional to the off-diagonal matrix element $\mathbf{M}_{\mathbf{k}}^{v c}$ turn out to cancel in the equation of $\rho_{\mathbf{k}}^{\lambda}$. The first order $S_{\mathbf{k}, \mathbf{q}(1)}^{\lambda_{1} \lambda_{2} j}$ describes the phonon-assisted intraband absorption, while the zeroth order $S_{\mathbf{k}, \mathbf{q}(0)}^{\lambda_{1} \lambda_{2} j}$ yields the standard Boltzmann-like equation containing phonon-assisted intra- and interband scattering processes. Using Eq. (4.4) and (4.5) the equation of motion for the carrier occupation $\rho_{\mathbf{k}}^{\lambda}$ can be written as

$$
\left.\dot{\rho}_{\mathbf{k}}^{\lambda}\right|_{H_{c, p}+H_{c, c}}=\left[\Gamma_{\lambda, \mathbf{k}}^{\text {in }}+\Gamma_{\lambda, \mathbf{k}}^{\text {in }, \mathrm{A}}\right]\left(1-\rho_{\mathbf{k}}^{\lambda}\right)-\left[\Gamma_{\lambda, \mathbf{k}}^{\text {out }}+\Gamma_{\lambda, \mathbf{k}}^{\text {out }, \mathrm{A}}\right] \rho_{\mathbf{k}}^{\lambda} .
$$

Here, $\Gamma_{\lambda, \mathbf{k}}^{\text {in,out }}$ describe the time- and momentum-dependent scattering rates including the Coulomb- and phonon-assisted contributions, which are discussed in detail in Section 2.4.2. The field-assisted second order contribution $\Gamma_{\lambda, \mathbf{k}}^{\mathrm{in}, \mathrm{A}}$, responsible for 
the intraband absorption, reads

$$
\begin{aligned}
& \Gamma_{\lambda, \mathbf{k}}^{\mathrm{in}, \mathrm{A}}=2 \pi \sum_{\lambda^{\prime} j \mathbf{q}} {\left[\left|g_{\mathbf{k}, \mathbf{q}}^{\lambda^{\prime} \lambda j}\right|^{2}\left(\frac{\tilde{M}_{\mathbf{k}, \mathbf{q}}^{\lambda^{\prime} \lambda}}{\Delta \varepsilon_{\mathbf{k}, \mathbf{q}}^{\lambda \lambda^{\prime}}} \delta\left(\Delta \varepsilon_{\mathbf{k}, \mathbf{q}}^{\lambda \lambda^{\prime}} \pm \hbar \omega_{L}\right)\right) \rho_{\mathbf{k}-\mathbf{q}}^{\lambda^{\prime}} n_{\mathbf{q}}^{j}\right.} \\
&\left.+\left|g_{\mathbf{k}+\mathbf{q}, \mathbf{q}}^{\lambda^{\prime} \lambda j}\right|^{2}\left(\frac{\tilde{M}_{\mathbf{k}+\mathbf{q}, \mathbf{q}}^{\lambda \lambda^{\prime}}}{\Delta \varepsilon_{\mathbf{k}+\mathbf{q}, \mathbf{q}}^{\lambda^{\prime} \lambda}} \delta\left(\Delta \varepsilon_{\mathbf{k}+\mathbf{q}, \mathbf{q}}^{\lambda^{\prime} \lambda} \pm \hbar \omega_{L}\right)\right) \rho_{\mathbf{k}+\mathbf{q}}^{\lambda^{\prime}}\left(n_{\mathbf{q}}^{j}+1\right)\right], \\
& \Gamma_{\lambda, \mathbf{k}}^{\mathrm{out}, \mathrm{A}}=2 \pi \sum_{\lambda^{\prime} j \mathbf{q}}\left[\left|g_{\mathbf{k}, \mathbf{q}}^{\lambda^{\prime} \lambda j}\right|^{2}\left(\frac{\tilde{M}_{\mathbf{k}, \mathbf{q}}^{\lambda^{\prime} \lambda}}{\Delta \varepsilon_{\mathbf{k}, \mathbf{q}}^{\lambda \lambda^{\prime}}} \delta\left(\Delta \varepsilon_{\mathbf{k}, \mathbf{q}}^{\lambda \lambda^{\prime}} \pm \hbar \omega_{L}\right)\right)\left(\rho_{\mathbf{k}-\mathbf{q}}^{\lambda^{\prime}}+1\right)\left(n_{\mathbf{q}}^{j}+1\right)\right. \\
&\left.+\left|g_{\mathbf{k}+\mathbf{q}, \mathbf{q}}^{\lambda^{\prime} \lambda j}\right|^{2}\left(\frac{\tilde{M}_{\mathbf{k}+\mathbf{q}, \mathbf{q}}^{\lambda \lambda^{\prime}}}{\Delta \varepsilon_{\mathbf{k}+\mathbf{q}, \mathbf{q}}^{\lambda^{\prime}}} \delta\left(\Delta \varepsilon_{\mathbf{k}+\mathbf{q}, \mathbf{q}}^{\lambda^{\prime} \lambda} \pm \hbar \omega_{L}\right)\right)\left(\rho_{\mathbf{k}+\mathbf{q}}^{\lambda^{\prime}}+1\right) n_{\mathbf{q}}^{j}\right]
\end{aligned}
$$

with $\tilde{M}_{\mathbf{k}, \mathbf{q}}^{\lambda \lambda^{\prime}}=i e_{0} / m_{0}\left(\mathbf{M}_{\mathbf{k}-\mathbf{q}}^{\lambda^{\prime} \lambda^{\prime}}-\mathbf{M}_{\mathbf{k}}^{\lambda \lambda}\right) \cdot \mathbf{A}(t)$. It accounts for optically driven intraband absorption processes that are assisted by phonon absorption or emission fulfilling the momentum and energy conservation. A detailed derivation of the field-assisted contribution can be found in the appendix E. Basically, these terms have a similar form as the scattering rates $\Gamma_{\lambda, \mathbf{k}}^{\text {in,out }}$. They are proportional to the optical matrix element $\tilde{M}_{\mathbf{k}, \mathbf{q}}^{\lambda \lambda{ }^{\prime}}$ and therefore a contribution to the DT is expected.

Similar to Eq. (4.2), Eq. (4.6) can be further simplified by using the ansatz

$$
\rho_{\mathbf{k}}^{\lambda}(t)=\rho_{\mathbf{k}}^{\lambda, 0}(t)+\delta \rho_{\mathbf{k}}^{\lambda}(t) .
$$

Here, $\rho_{\mathbf{k}}^{\lambda, 0}(t)$ contains the pump-induced occupation resulting from the Coulomband phonon-assisted scattering rates $\Gamma_{\lambda, \mathbf{k}}^{\text {in,out }}\left[41\right.$, while $\delta \rho_{\mathbf{k}}^{\lambda}(t)$ describes a small perturbation induced by the probe pulse. The latter is in particularly driven by phonon-assisted intraband absorption processes. Assuming a weak $\delta$-shaped probe pulse $\mathbf{A}=\mathbf{A}_{0} \delta(t-\tau)$, the Fourier transform for the perturbation yields

$$
\delta \rho_{\mathbf{k}}^{\lambda}(\omega, \tau)=\frac{\Gamma_{\lambda, \mathbf{k}}^{\mathrm{in}, \mathrm{A}}(\omega, \tau)\left(1-\rho_{\mathbf{k}}^{\lambda, 0}(\tau)\right)-\Gamma_{\lambda, \mathbf{k}}^{\mathrm{out}, \mathrm{A}}(\omega, \tau) \rho_{\mathbf{k}}^{\lambda, 0}(\tau)}{i \omega+\tilde{\gamma}}
$$

where the small damping $\tilde{\gamma}=\Gamma_{\lambda, \mathbf{k}}^{\mathrm{in}}+\Gamma_{\lambda, \mathbf{k}}^{\text {out }}$ is assumed to be constant. Note that this quantity has only a marginal influence on the impact of intraband absorption processes since all different wave number contributions have to be summed up, cf. Eq. 4.12). The expression of $\delta \rho_{\mathbf{k}}^{\lambda}(\omega, \tau)$ neglects the non-linear terms proportional to $\mathbf{A}(t)$ assuming a weak probe pulse. 


\subsection{Transient negative differential transmission}

The absorption coefficient $\alpha(\omega)$ of graphene is determined by the imaginary part of the optical susceptibility $\chi(\omega)=j(\omega) /\left(\epsilon_{0} \omega^{2} A(\omega)\right)[41$. The current reads

$$
\mathbf{j}(t)=\frac{2 e_{0} \hbar}{m_{0} L^{2}} \sum_{\mathbf{k}}\left[2 \mathbf{M}_{\mathbf{k}}^{v c} \operatorname{Im}\left[p_{\mathbf{k}}\right]-i \sum_{\lambda} \mathbf{M}_{\mathbf{k}}^{\lambda \lambda} \rho_{\mathbf{k}}^{\lambda}+\sum_{\lambda} \frac{e_{0}}{\hbar} \mathbf{A}(t) \rho_{\mathbf{k}}^{\lambda}\right]
$$

containing an interband (first term) and an intraband contribution (second and third term). Within the linear band regime, it can be shown that the third term of the current $\mathbf{j}(t)$ in Eq. 4.11) cancels with the real part of interband term resulting in a purely imaginary current in the Fourier space [91|. Finally, the absorption coefficient can be written as

$$
\alpha(\omega)=\frac{2 e_{0} \hbar}{m_{0} L^{2} \omega c_{0} \varepsilon_{0}} \sum_{\mathbf{k}} \operatorname{Im}\left[\frac{2 \mathbf{M}_{\mathbf{k}}^{v c} \cdot \hat{\mathbf{e}}_{A} \operatorname{Im}\left[p_{\mathbf{k}}(\omega)\right]}{A(\omega)}-i \sum_{\lambda} \frac{\mathbf{M}_{\mathbf{k}}^{\lambda \lambda} \cdot \hat{\mathbf{e}}_{A} \rho_{\mathbf{k}}^{\lambda}(\omega)}{A(\omega)}\right]
$$

with the direction of polarization of the vector potential $\hat{\mathbf{e}}_{A}$. The interband contribution is proportional to $\mathbf{M}_{\mathbf{k}}^{v c}$ and to the microscopic polarization $p_{\mathbf{k}}(\omega)$ leading to the well-known absorption spectrum of graphene with a constant value in the visible spectral range [6, 41, 66, 92, 94, , see Chapter 3 . The phonon-assisted intraband processes influence the spectra via the carrier occupation probability $\rho_{\mathbf{k}}^{\lambda}(\omega)$ weighted by the optical intraband matrix element $\mathbf{M}_{\mathbf{k}}^{\lambda \lambda}$.

Figure 4.2 shows the calculated free-particle absorption spectrum including only the phonon-induced intraband processes within the linear regime of the bandstructure. Within the linear optics, the appearing carrier occupations in $\delta \rho_{\mathbf{k}}^{\lambda}(\omega)$ are approximated by Fermi-Dirac distributions at room temperature. An enhanced absorption in the range of $1 \%$ is observed compared to the spectrum containing only interband transitions. The effect is rather small since the small amount of excited carriers due the Fermi-Dirac distributions at room temperature, which results in a weak efficiency of the phonon-assisted intraband processes. Additionally, the calculations reveal that the higher the photon energy, the stronger is the contribution of the intraband absorption. This can be traced back to the increasing density of states providing more scattering partners at higher photon energies $\hbar \omega_{L}$.

Since the main goal in this work is the description of the non-equilibrium regime, the focus lies on the differential transmission spectrum that is determined by $\Delta T / T_{0}(\tau, \omega) \propto \alpha^{(t)}(\omega)-\alpha^{(p, t)}(\omega, \tau)$. Assuming an isotropic carrier distribution 


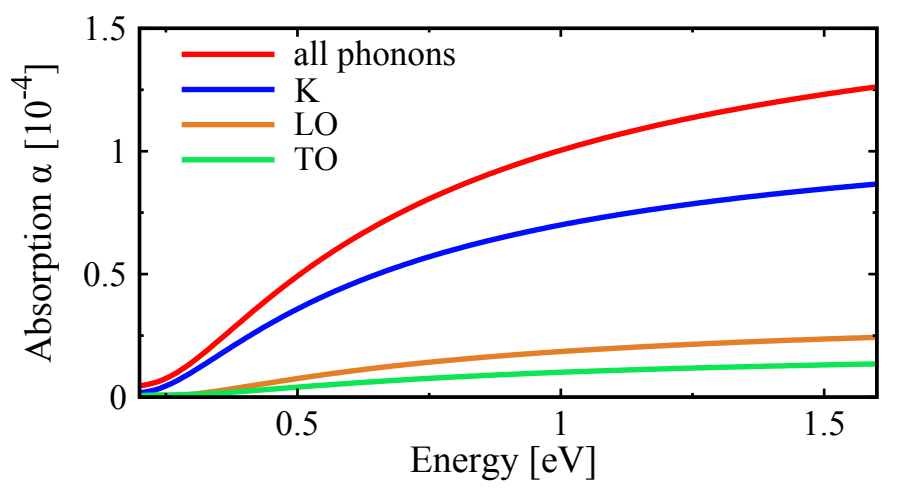

Figure 4.2.: The free-particle absorption spectrum of graphene including only the contribution of the intraband absorption. The figure illustrates the contribution of different optical phonon modes and reveals that the phonon-induced intraband processes lead to a slightly enhanced absorption.

in the momentum space after several fs [28], the transient DT reads [56]

$$
\begin{aligned}
\Delta T / T_{0}(\tau, \omega) \propto & \frac{\left|\mathbf{M}_{k_{\omega}}^{v c} \cdot \hat{\mathbf{e}}_{A}\right|^{2} e_{0} \hbar^{2}}{8 \pi m_{0} v_{F}^{2}}\left[\Delta \rho_{k_{\omega}}^{(p, t)}(\tau)-\Delta \rho_{k_{\omega}}^{(t)}(\tau)\right] \\
& +\frac{i}{L^{2} \omega A_{0}^{(t)}} \sum_{\lambda \mathbf{k}} \mathbf{M}_{\mathbf{k}}^{\lambda \lambda} \cdot \hat{\mathbf{e}}_{A} \operatorname{Im}\left[\rho_{\mathbf{k}}^{\lambda,(p, t)}(\omega, \tau)-\rho_{\mathbf{k}}^{\lambda,(t)}(\omega, \tau)\right],
\end{aligned}
$$

with the excitation momentum $k_{\omega}=\hbar \omega_{L} / 2 v_{F}$. The first line of Eq. (4.13) accounts for interband transitions, which reflects the absorption bleaching due to the additional carriers lifted from the valence into the conduction band by the pump pulse. As a result, the transient DT is expected to show a positive peak during the excitation. The second line describes the phonon-assisted intraband absorption processes that are induced by the probe pulse. Since these processes lead to an increased absorption after the excitation a negative contribution to the transient DT signal is expected.

Inserting Eq. 4.10 into Eq. 4.13), the impact of phonon-assisted intraband absorption processes on the transient differential transmission (Fig. 4.3) can be determined. As initial condition, a Fermi distribution for the electron population $\rho_{\mathbf{k}}$ and a Bose-Einstein distribution for the phonon occupations $n_{\mathbf{q}}^{j}$ at room temperature is assumed. First, the impact of the excitation strength on the transient DT spectrum is analyzed. Figure 4.3 shows the calculated degenerate transient DT spectrum $\Delta T / T_{0}(\tau)$ excited by a $50 \mathrm{fs}$ pulse with a pump fluence of $8 \mu \mathrm{Jcm}^{-2}$ at the photon energy $E=1.5 \mathrm{eV}$. The abrupt increase of the signal reflects the ultrafast optical injection of non-equilibrium carriers around the photon energy resulting in 
Chapter 4. Impact of intraband absorption in graphene

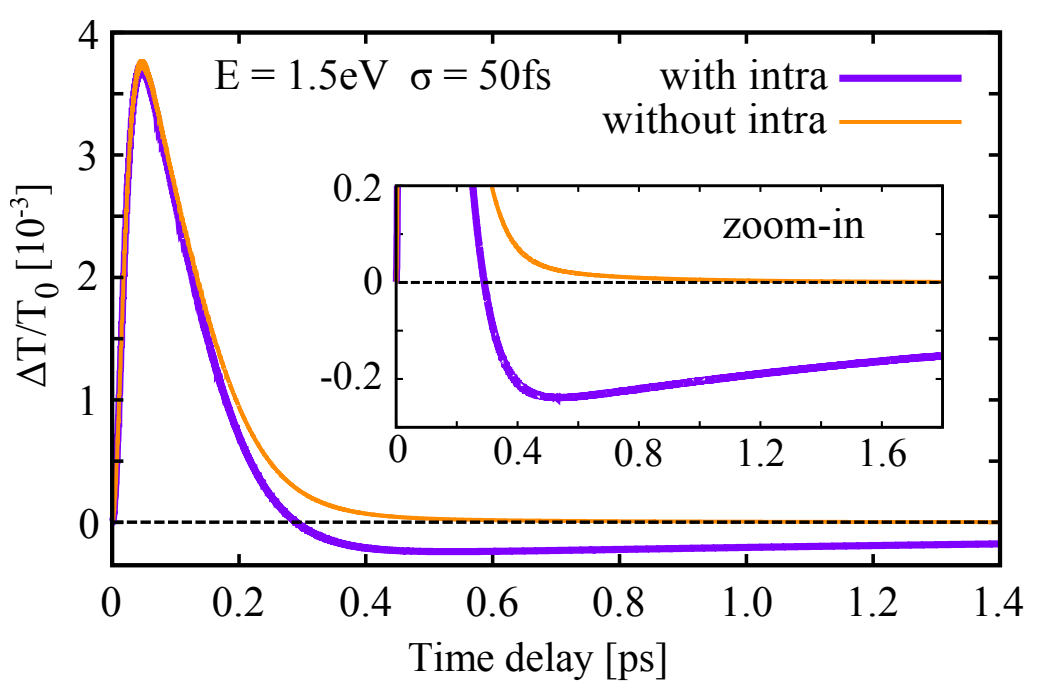

Figure 4.3.: Transient differential transmission spectrum as a function of the delay time $\tau$ between the pump and the probe pulse illustrating the impact of phonon-assisted intraband processes, which account for a zero-crossing. Figure is adopted from $[56]$.

an absorption bleaching. The transmission of the probe pulse is enhanced after the pump pulse has excited carrier from the valence into the conduction band, which explains the increase of the DTS during the optical excitation. Afterwards, the signal decreases on a fs timescale due to the carrier-carrier (thermalization of the non-equilibrium electrons) and carrier-phonon (cooling and recombination of the thermalized electrons) scattering $[22$. Taking into account only interband absorption (Fig. 4.3, thin line), the transient DT signal remains positive during the entire dynamics. However, after including the phonon-assisted intraband absorption, the transient exhibits a distinct zero-crossing after approximately $310 \mathrm{fs}$ followed by a subsequent recovery towards zero on a ps timescale. This observation reflects well the experimental observation, as will be discussed below.

For both theory curves in Fig. 4.3, a bi-exponential decay is found characterized by a fast time constant $\tau_{1}=90 \mathrm{fs}$ and a slow component $\tau_{2}^{\text {inter }}=0.6 \mathrm{ps}$ (positive DT, thin orange line) and $\tau_{2}^{\text {inter+intra }}=2.8 \mathrm{ps}$ (negative DT, thick violet line) depending on whether intraband processes are included or not. The intraband absorption does not change the first decay rate, whereas the second decay is considerably slowed down in the presence of intraband absorption. The decay $\tau_{2}^{\text {inter }}$ is determined by the electron cooling due to electron-phonon interaction, whereas the decay $\tau_{2}^{\text {inter+intra }}$ in the presence of intraband absorption has a different origin: Since intraband absorption occurs always for finite carrier population $\rho_{\mathbf{k}}^{\lambda}, \tau_{2}^{\text {inter+intra }}$ is limited by 
carrier recombination processes that take place on a slower time scale (several ps) than the carrier cooling (one ps).

Figure 4.4(a) illustrates the impact of the phonon-assisted intraband absorption on the transient DT at different pump fluences covering the range of $2-24 \mu \mathrm{Jcm}^{-2}$. The fast decay time $\tau_{1}$ exhibits a weak increase from 80 to $110 \mathrm{fs}$ with the increasing fluence. In the case of weak excitation, the thermalized carrier distribution differs only slightly from the initial thermal distribution. Due to the low population, the impact of Pauli blocking above the thermal tail is almost negligible with the consequence that the out-scattering from the excited states becomes very efficient and leads to a fast decay $\tau_{1}$. For stronger excitation, the efficient Pauli blocking suppresses the out-scattering explaining the increased $\tau_{1}$ time 95 . In contrast to the fast decay component, the slower $\tau_{2}$ time decreases approximately linearly from 3.3 to $2.7 \mathrm{ps}$ with increasing pump fluence: At high excitation, there is an increased number of excited carriers at the Dirac-point resulting in faster recombination of the carrier system.

To test the predicted behaviour, the results were compared with recently performed experimental data 26] depicted in Fig. 4.4(b). Investigating increasing pump intensity, one can see both in theory and experiment similar timescales $\tau_{1}$ and $\tau_{2}$. Furthermore, the same trend of the zero-crossing, and height trend as a function of pump intensity is found. The absolute value of the negative transient DT signal increases with the pump fluence, cp. the insets of Fig. 4.4(a) and (b). At high fluences, the intraband absorption is enhanced due to the larger number of available carriers in the conduction band. At the same time, increasing the fluence gives rise to a more efficient Pauli blocking, which increases the absorption bleaching and leads to a stronger positive DT signals. Figure 4.4(c) shows the fluence-dependence of the ratio $\mathrm{I}^{-}{ }_{\max } / \mathrm{I}^{+}{ }_{\max }$ between the maximal negative $\left(\mathrm{I}^{-}{ }_{\max }\right)$ and positive $\left(\mathrm{I}^{+}{ }_{\max }\right)$ transient DT signal. The maxima have been extracted from the noisy experimental data by performing a fitting that takes into account the shape of the laser pulse and the exponential decay with different time constants. A good qualitative agreement between the theoretically predicted and experimentally measured behaviour is found: The ratio clearly decreases with the fluence with a maximal ratios in the range of $10 \%$ found in the weak excitation regime. The decrease can be traced back to the predominant role of the Pauli blocking at high fluences prevailing over the increased efficiency of intraband absorption. This also explains the observation both in theory and experiment that the zero crossing occurs at larger delay times with the increasing fluence, $\mathrm{cf}$. the insets of Fig. 4.4(a) and (b). The theoretically predicted values for $\tau_{2}$ correspond well with the timescale experimentally observed.

The appearing quantitative deviations with respect to the more pronounced temporal shift of the zero-crossing for increasing fluences and with respect to the higher relative strength of the negative DT spectrum at low fluences might be due 
to the neglected effects of Coulomb-induced intraband absorption processes and energy renormalization. In particular, the Coulomb-assisted processes are expected to become important in the high excitation regime and could explain the more pronounced fluence-dependence of the temporal zero-crossing in the experimental DT signal. The influence of substrate-induced doping and sample impurities might also responsible for the quantitative differences to the experiment. 
Chapter 4. Impact of intraband absorption in graphene
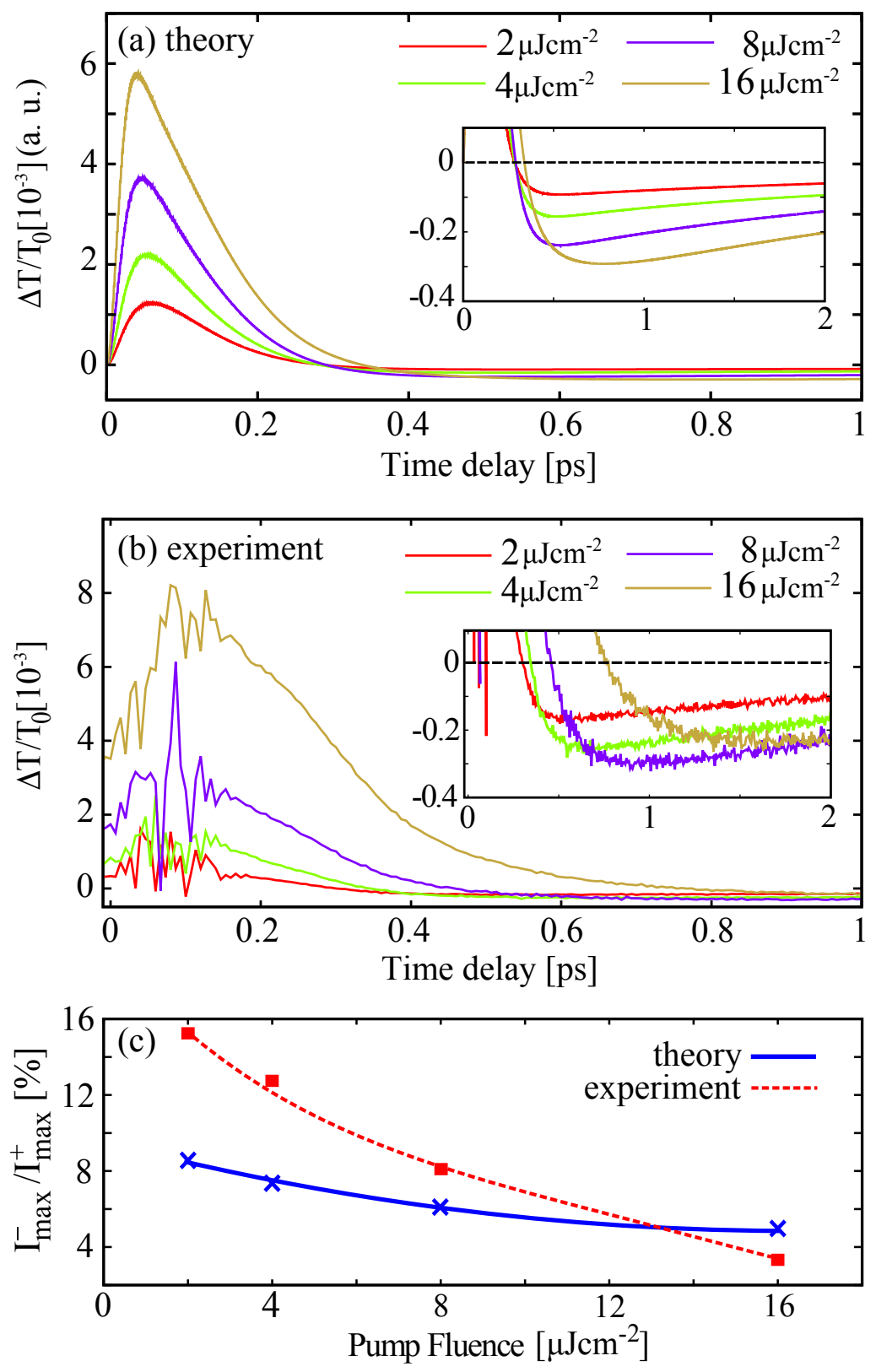

Figure 4.4.: (a) Transient differential transmission spectrum for different pump fluences including the phonon-assisted intraband absorption processes. (b) Experimentally measured transient differential transmission at the same conditions (excitation energy of $1.5 \mathrm{eV}$ and a pulse width of $50 \mathrm{fs}$ ). (c) Fluence-dependent ratio $\mathrm{I}^{-}{ }_{\max } / \mathrm{I}^{+}{ }_{\max }$ denoting the maximal negative and positive transient DT signal, respectively. The strongest relative impact of phonon-assisted intraband processes is found at small fluences - in good agreement with the experiment. Figure is taken from $\lceil 56]$. 


\section{Terahertz carrier relaxation dynamics in graphene}

The carrier dynamics in the Terahertz (THz) region exhibit a number of features that have been interpreted in the framework of phenomenological models, but have not been understood quantitatively as well as qualitatively in terms of a fundamental microscopic theory of many-particle processes. For example, the photoinduced $\mathrm{THz}$ conductivity may be either positive or negative. The positive photoinduced $\mathrm{THz}$ conductivity has been viewed in the context of simple Drude models as stemming from enhanced free-carrier intraband absorption upon photoexcitation $[82,96$, whereas the negative photoinduced $\mathrm{THz}$ conductivity has been attributed variously to stimulated $\mathrm{THz}$ emission [97], enhanced carrier scattering with optical phonons, surface optical phonons or charge impurities 98 -100], and carrier heating [101-103]. In the region near to the Dirac point, hot-carrier relaxation and cooling slow down considerably due to the vanishing density of states, the energetic mismatch with the energy of optical phonons, and the weak scattering with acoustic phonons $23,55,104 \mid$. Disorder-assisted electron-phonon (supercollision) scattering processes have been recently proposed to account for the experimentally observed cooling times in the THz region [105], but the underlying enhancement of the phase space restrictions should strongly depend on the quality and the degree of disorder of the particular graphene sample. Hence, both a microscopic theoretical treatment and a methodical experimental investigation are needed to provide a rigorous foundation for understanding the $\mathrm{THz}$ dynamics of hot carriers in graphene.

In this chapter the ultrafast time-resolved $\mathrm{THz}$ spectroscopy is used to systematically investigate the hot-carrier dynamics for pristine and highly doped graphene. To model the pump-probe experiments the microscopic density-matrix theory including all relevant time-dependent carrier-carrier and carrier-phonon scattering mechanisms is used, which explicitly account for the time-dependent interaction of the carriers with the optical and $\mathrm{THz}$ field. The observed dynamics can be accounted qualitatively without including any extrinsic effects such as disorder, and without using any free parameters. Furthermore, the results will be compared with the standard Drude model. 


\subsection{Terahertz carrier dynamics}

In the following, the results of a coordinated theoretical and experimental program is presented, using ultrafast time-resolved $\mathrm{THz}$ spectroscopy [106] combined with microscopic modelling 41, 107 to systematically study the $\mathrm{THz}$ carrier dynamics in a wide variety of graphene samples, including epitaxial and chemical-vapordeposited (CVD) graphene 107.

\subsubsection{Hot-carrier dynamics in graphene}

A schematic illustration of the hot-carrier dynamics in graphene following ultrafast photoexcitation appears in Figure 5.1, which shows how they depend significantly on the Fermi level. It is important to distinguish between carrier thermalization via carrier-carrier scattering, which only redistributes the deposited energy within the electron gas, and carrier cooling via carrier-phonon scattering, which takes energy from the electron gas into the lattice. Figure 5.1(a) illustrates the hot-carrier relaxation and cooling dynamics in highly doped graphene. Initially, the ultrafast optical pump pulse injects high-energy non-equilibrium electrons in the conduction band and holes in the valence band. The strong intraband and interband carrier-carrier scattering processes lead to ultrafast carrier relaxation and thermalization which establish a single uniform hot-carrier Fermi-Dirac distribution within $\sim 100-200 \mathrm{fs}$ after photoexcitation [108]. At that point, carrier generation (through impact ionization) and carrier relaxation (through Auger recombination and intraband carrier-carrier scattering) processes just balance each other, while hot carriers cool via optical and acoustic phonon emission. The hot-carrier cooling is facilitated by these efficient Coulomb interactions by continuously rethermalizing the hot-carrier distribution and replenishing the carriers at high energies.

The situation in undoped (or very lightly doped) graphene is very different, as shown in Figure 5.1(b). Immediately after photoexcitation, the phase space for impact ionization processes is large, while Auger recombination processes are inhibited, which leads to a significant carrier multiplication in the conduction band up to a moderate excitation regime [30,35]. Again, the strong intraband and interband carrier-carrier scattering processes establish a single uniform hot-carrier Fermi-Dirac distribution on an ultrafast timescale followed by hot-carrier cooling via optical and acoustic phonon emission. At later times, the small phase space near the Dirac point strongly reduces the efficiency of carrier rethermalization via the Coulomb interactions, which slows down phonon emission and hot-carrier cooling. 


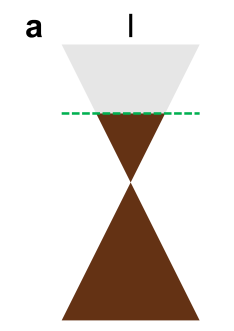

b

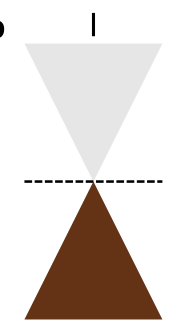

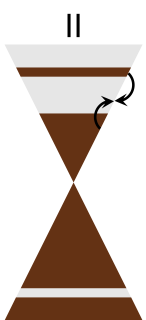

II

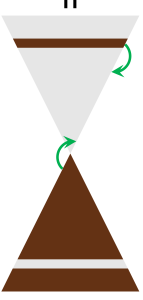

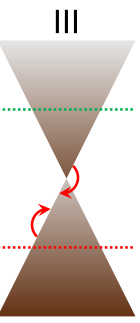

III

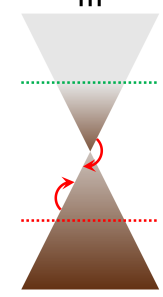

IV

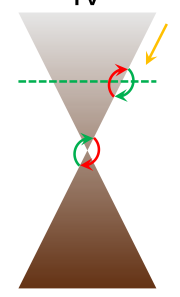

IV

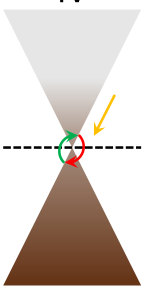

Figure 5.1.: Hot-carrier relaxation and cooling dynamics of undoped and highly n-doped graphene. (a) Hot-carrier relaxation and cooling dynamics of highly n-doped graphene (step I). Initially, the optical pump pulse injects hot non-equilibrium carriers at high energies (step II). The hot electrons and holes thermalize within the conduction and valence bands, respectively, due to very efficient intraband carrier-carrier scattering processes (step III). As the hot carriers relax to lower energies, interband Auger recombination processes become allowed that quickly merge the separate electron and hole quasi-Fermi levels and lead to a single uniform hot-carrier Fermi-Dirac distribution within $\sim 100-200$ fs after photoexcitation (step IV). The hot carriers cool further via optical phonon emission facilitated by very efficient carrier-carrier rethermalization in highly doped graphene. (b), Hot-carrier relaxation and cooling dynamics of undoped graphene (step I). Initially, the optical pump pulse injects hot non-equilibrium carriers at high energies (step II). In contrast to (a), interband impact ionization processes are possible and lead for a moderate excitation regime to a significant carrier multiplication in the conduction band. Again, as the hot carriers relax to lower energies, interband Auger recombination processes become allowed (step III) that leads to a single uniform hot-carrier Fermi-Dirac distribution within $\sim 100-200$ fs after photoexcitation (step IV). The hot carriers cool further also via optical phonon emission, but carrier-carrier rethermalization in undoped graphene becomes increasingly inefficient at low energies due to the small phase space near the Dirac point. Figure is taken from [107]. 


\subsubsection{Perturbation approach for the $\mathrm{THz}$ dynamics}

The dynamic $\mathrm{THz}$ response due to an optical excitation can be microscopically addressed by evaluating the graphene Bloch equations [17]:

$$
\begin{aligned}
& \frac{d}{d t} \rho_{\mathbf{k}}^{\lambda}=-\frac{e_{0}}{\hbar} \mathbf{E} \cdot \nabla_{\mathbf{k}} \rho_{\mathbf{k}}^{\lambda}+\sigma_{\lambda} 2 \operatorname{Im}\left[\Omega_{\mathbf{k}}^{v c *} p_{\mathbf{k}}\right]+\Gamma_{\lambda \mathbf{k}}^{\text {in }}\left[1-\rho_{\mathbf{k}}^{\lambda}\right]-\Gamma_{\lambda \mathbf{k}}^{\text {out }} \rho_{\mathbf{k}}^{\lambda}, \\
& \frac{d}{d t} p_{\mathbf{k}}=\left[i \Delta \omega_{\mathbf{k}}-\gamma_{\mathbf{k}}\right] p_{\mathbf{k}}-i \Omega_{\mathbf{k}}^{v c}\left[\rho_{\mathbf{k}}^{c}-\rho_{\mathbf{k}}^{v}\right] .
\end{aligned}
$$

Here, a drift term $\mathbf{E} \cdot \nabla_{\mathbf{k}} \rho_{\mathbf{k}}^{\lambda}$ expressing the light-induced intraband transitions is included, which is crucial for the $\mathrm{THz}$ dynamics driven by the probe pulse. The explicit form of the many-particle contributions as well as the equation for the phonon dynamics can be found in Chapter 2. Assuming a weak THz probe pulse, the occupation $\rho_{\mathbf{k}}^{\lambda}(t)$ can be treated perturbatively:

$$
\rho_{\mathbf{k}}^{\lambda}(t)=\rho_{\mathbf{k}}^{\lambda, 0}(t)+\delta \rho_{\mathbf{k}}^{\lambda}(t)
$$

where $\rho_{\mathbf{k}}^{\lambda, 0}(t)$ is the pump pulse-induced occupation, while $\delta \rho_{\mathbf{k}}^{\lambda}(t)$ describes the weak carrier occupation excited by the $\mathrm{THz}$ probe pulse.

To obtain the dynamic $\mathrm{THz}$ response from the differential $\mathrm{THz}$ transmission spectra, the fact is exploit that ultrafast carrier-carrier scattering in graphene forms a uniform Fermi-Dirac distribution within the first tens of femtoseconds after the excitation [22] and the subsequent dynamics is fully characterized by the temporal evolution of the transient temperature $T_{\lambda}(t)$ and transient Fermi level $\varepsilon_{F}^{\lambda}(t)$ of electrons and holes. Thus, by iteratively evaluating $T_{\lambda}(t)$ and $\varepsilon_{F}^{\lambda}(t)$ on the basis of the numerically calculated carrier dynamics at each time step, the pump pulse-induced occupation $\rho_{\mathbf{k}}^{\lambda, 0}(t)$ can be obtained. The dynamics of the probe pulse-induced carrier occupation is given by a separate equation of motion

$$
\frac{d}{d t} \delta \rho_{\mathbf{k}}^{\lambda}(t)=-\frac{e_{0}}{\hbar} \mathbf{E} \cdot \nabla_{\mathbf{k}} \rho_{\mathbf{k}}^{\lambda, 0}(t)-\Gamma_{\lambda \mathbf{k}}^{0}(t) \delta \rho_{\mathbf{k}}^{\lambda}(t)
$$

where $\Gamma_{\lambda \mathbf{k}}^{0}(t)=\Gamma_{\lambda \mathbf{k}}^{\mathrm{in}, 0}(t)+\Gamma_{\lambda \mathbf{k}}^{\mathrm{out}, 0}(t)$ is the diagonal contribution stemming from the Boltzmann-like scattering terms (cf. Eq. 5.1), which are independent of the probe pulse as denoted by the index 0 . Non-linear contributions in the probe pulse and non-diagonal terms have been neglected here. For the response of the $\mathrm{THz}$ probe field, the interband contribution is negligibly small, since the intraband processes are dominating. By inserting the numerically evaluated Fourier transform of $\delta \rho_{\mathbf{k}}^{\lambda}(t)$ in Eq. 3.3, the Coulomb- and phonon-assisted dynamics induced by the THz probe pulse can be determined. More details of the derivation of the $\mathrm{THz}$ dynamics can be found in appendix $\mathrm{F}$. 


\subsection{Optical pump and $\mathrm{THz}$ probe differential transmission spectra}

To study the dynamic $\mathrm{THz}$ response of graphene, the ultrafast time-resolved $\mathrm{THz}$ spectroscopy 106, 109, 110 is used, which has established itself as a very powerful all-optical experimental technique for directly probing the relaxation and cooling dynamics of photoexcited carriers, because it is sensitive to both the number of carriers and their distribution in energy. The details of the experimental set-up are provided in Ref. [107].

To obtain the optical pump and THz-probe DTS, the intraband absorption coefficient has to be determined, which is given by

$$
\alpha(\omega) \propto \frac{1}{\omega} \sum_{\lambda, \mathbf{k}} \operatorname{Im}\left[i \frac{\mathbf{M}_{\mathbf{k}}^{\lambda \lambda} \cdot \hat{\mathbf{e}}_{A} \delta \rho_{\mathbf{k}}^{\lambda}(\omega)}{A(\omega)}\right] .
$$

Inserting the numerically evaluated Fourier transform of $\delta \rho_{\mathbf{k}}^{\lambda}(t)$, the DTS can be calculated via Eq. 2.78.

Experimental samples The experimental samples vary the type of graphene and the degree of disorder, the Fermi level, the number of graphene layers and their stacking orientation, the substrate temperatures and initial carrier temperatures and the type of underlying substrate to determine the dominant mechanisms responsible for the hot-carrier relaxation and cooling dynamics for different graphene material parameters and under different experimental conditions [107].

The experimental graphene sample is synthesized by using three different methods. The first type is multilayer epitaxial graphene (MEG), where the individual layers are electronically decoupled due to their unique rotational stacking, and each layer exhibits a single graphene layer Dirac cone near the Dirac point so that MEG behaves in essence as multilayer graphene 111, 112. The second type is singlecrystal CVD graphene (sCVDG), which is grown on oxygen-rich copper foil into large individual single crystals exceeding hundreds of micrometers in size [113. The last type is polycrystalline CVD graphene (pCVDG), which is grown on copper foil into large continuous layers with domain sizes on the order of hundreds of nanometers [114].

Since the different graphene samples are synthesized using completely different techniques, their Fermi level and degree of disorder are also very different. This allows to study the THz dynamics for different doping levels and different degrees of disorder. The MEG samples are highly n-doped $\left(\varepsilon_{F} \sim 100-400 \mathrm{meV}\right)$ due to electron transfer from the interface, and the Fermi level in subsequent layers decreases exponentially away from the substrate to around $\varepsilon_{F} \sim 10 \mathrm{meV}$ 81, 85, 115, 116. 
Chapter 5. Terahertz carrier relaxation dynamics in graphene

The CVDG samples are highly p-doped $\left(\varepsilon_{F} \sim 200-400 \mathrm{meV}\right)$ due to water vapor adsorption from the environment [117]. Thus, the dynamic $\mathrm{THz}$ response of graphene can directly compared with Fermi level far above, far below and very close to the Dirac point [107.

\subsubsection{Highly doped graphene}

First, epitaxial and CVD graphene with high doping density is considered. Figure $5.2(\mathrm{a}, \mathrm{b})$ shows the differential $\mathrm{THz}$ transmission signals at the peak of the $\mathrm{THz}$ probe pulse normalized to the $\mathrm{THz}$ transmission without photoexcitation $(\Delta t / t)$ as a function of pump-probe delay, for variable pump fluence and for variable substrate temperature, respectively, for an MEG sample with $\sim 3$ layers. The secondary peak in the differential $\mathrm{THz}$ transmission at $\sim 7 \mathrm{ps}$ is due to a roundtrip reflection of the optical pump pulse inside the substrate that photoexcites additional carriers [107|. Similar results are obtained for the other two types of the graphene samples [107]. Based on extensive measurements on many graphene samples under various experimental conditions, the $\mathrm{THz}$ carrier dynamics of all graphene samples with high doping are strikingly similar. The differential $\mathrm{THz}$ transmission is positive under all experimental conditions, which corresponds to a pump-induced increase of the $\mathrm{THz}$ transmission or a decrease of the THz absorption. This observation has been previously phenomenologically attributed to an increase of the carrier scattering rate and a corresponding decrease of the $\mathrm{THz}$ conductivity of graphene as the carrier temperature is raised upon photoexcitation, which is essentially a metal-like behaviour $98-100]$. In addition, the THz carrier dynamics are weakly dependent on the pump fluence and completely independent of the substrate temperature.

Performing phenomenological fits to the data in Figure $5.2(\mathrm{a}, \mathrm{b})$ the differential $\mathrm{THz}$ transmission follows closely a mono-exponential relaxation form under all experimental conditions. A summary of the extracted carrier relaxation times as a function of pump fluence and substrate temperature, respectively, for all graphene samples with high doping is presented in Figure 5.3 (a,b). The relaxation times of all graphene samples with high doping are weakly dependent on the pump fluence and completely independent of the substrate temperature. In addition, they are very similar in magnitude and in the range of $\sim 1-2$ ps with sample-to-sample variation within $\sim 20-30 \%$. On average, MEG samples exhibit slightly longer relaxation times than sCVDG and pCVDG samples, which can be attributed to a degree of disorder arising from charge impurities, substrate roughness, wrinkling and breaking of the transferred CVDG samples that can provide additional parallel channels for carrier cooling [105, 118, 119. However, the relaxation times of some CVDG samples can approach or exceed these of MEG samples, indicating that disorder-assisted electron-phonon (supercollision) cooling is not the dominant 
cooling mechanism in our high quality graphene samples, but generally provides at most only a modest correction.

Figure $5.2(\mathrm{c}, \mathrm{d})$ shows the calculated $\mathrm{THz}$ carrier dynamics for highly doped graphene with $\varepsilon_{F}=300 \mathrm{meV}$ under similar experimental conditions. The theoretical results reproduce all trends observed in the experiments. The hot-carrier $\mathrm{THz}$ dynamics results from the interplay between very efficient carrier-carrier scattering and carrier-optical-phonon scattering. The physical reason for the negligible substrate temperature dependence of the differential $\mathrm{THz}$ transmission is that the direct $\mathrm{THz}$ absorption itself is insensitive to the substrate temperature for highly doped graphene at least up to room temperature. Similarly, Figure 5.3(c,d) illustrates the calculated carrier relaxation times to directly compare with the experiments. The slight increase of the relaxation times with the pump fluence is due to the re-absorption of hot optical phonons generated during the initial carrier thermalization [17,41]. 

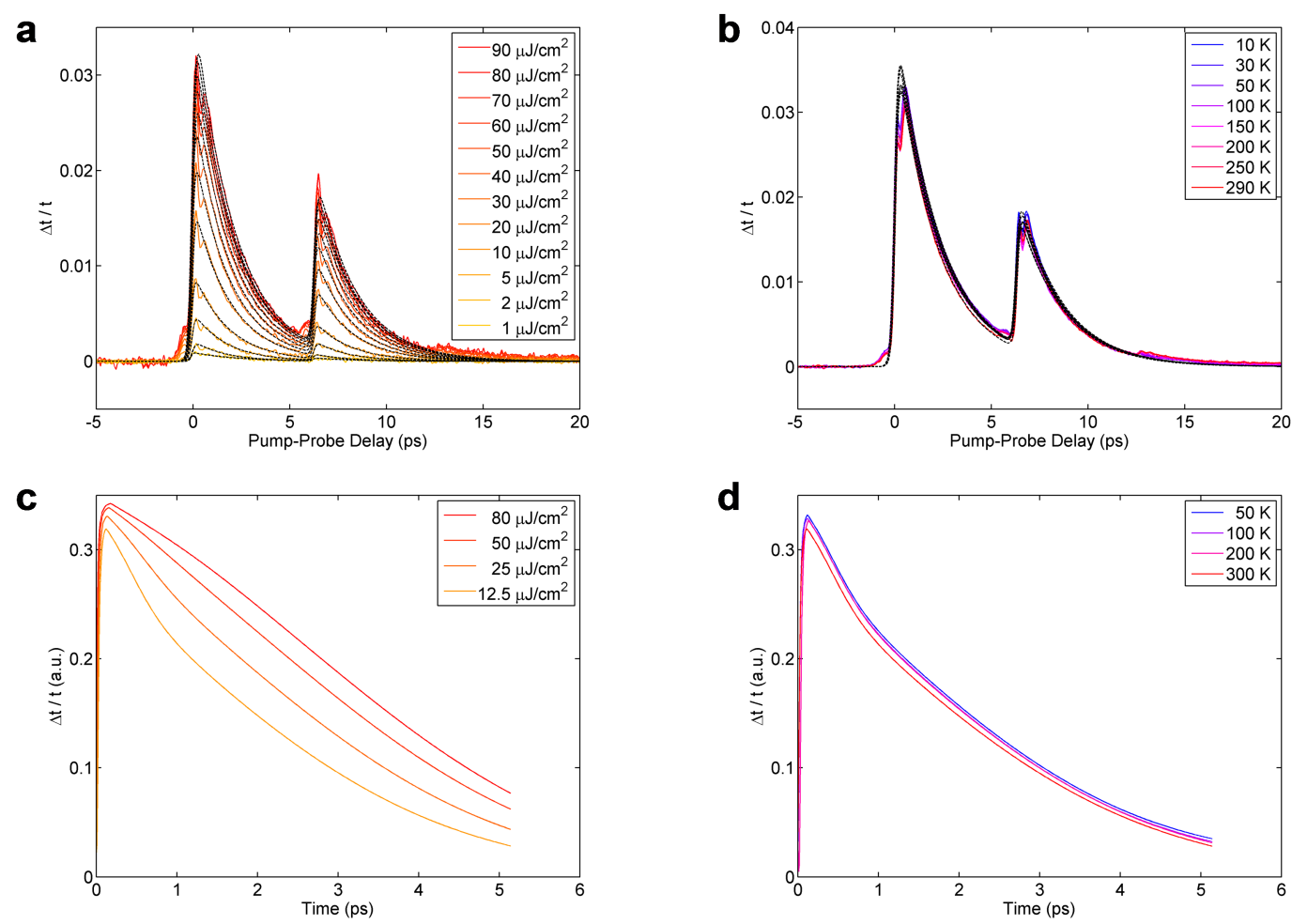

Figure 5.2.: Optical pump and $\mathrm{THz}$ probe DTS of highly doped graphene. Experimental differential $\mathrm{THz}$ transmission $\Delta t / t$ at the peak of the $\mathrm{THz}$ probe pulse as a function of pump-probe delay recorded at a substrate temperature of $300 \mathrm{~K}$ for a few different pump fluences (a) and at a pump fluence of $60 \mu \mathrm{Jcm}^{-2}$ for a few different substrate temperatures (b) for a highly doped MEG sample with $\sim 3$ layers. The THz carrier dynamics follow a fast mono-exponential relaxation at all substrate temperatures and all pump fluences. Theoretical differential $\mathrm{THz}$ transmission $\Delta t / t$ at a substrate temperature of $300 \mathrm{~K}$ for a few different pump fluences (c) and at a pump fluence of $12.5 \mu \mathrm{Jcm}^{-2}$ for a few different substrate temperatures (d) for highly doped graphene with $\varepsilon_{F}=300 \mathrm{meV}$. Theory and experiment are in excellent agreement under all conditions. Figure is adopted from [107|. 


\section{a}

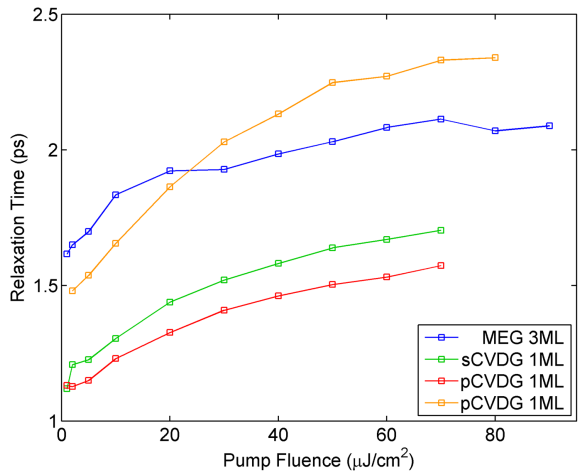

C

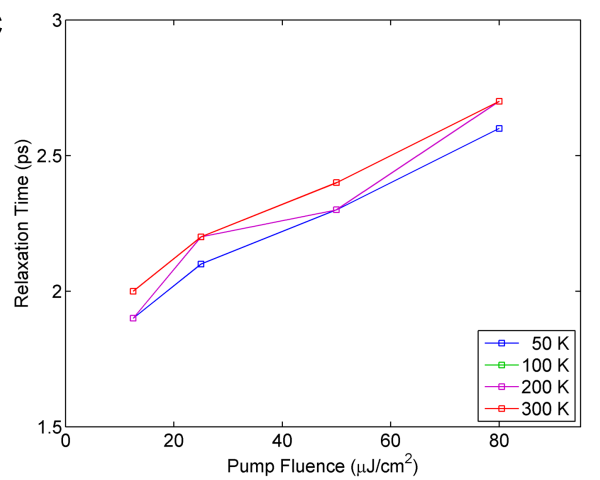

b

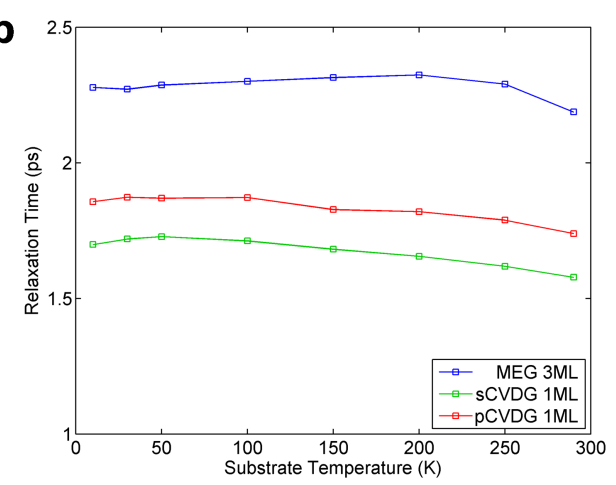

d

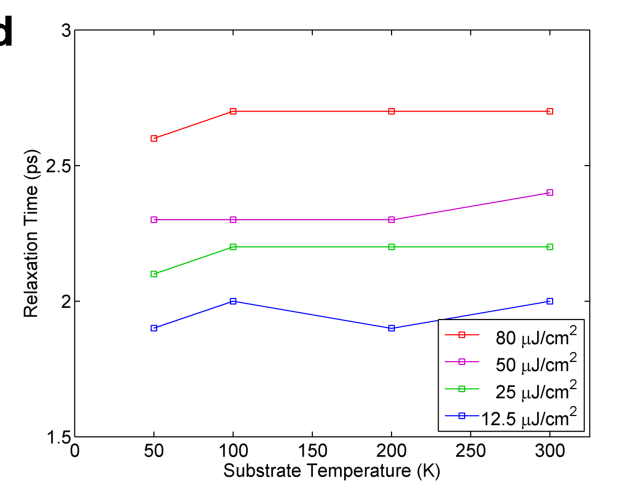

Figure 5.3.: $\mathrm{THz}$ carrier relaxation times in highly doped graphene. Carrier relaxation times extracted from fits to experimental differential $\mathrm{THz}$ transmission $\Delta t / t$ as a function of pump fluence at a substrate temperature of $300 \mathrm{~K}$ (a) and as a function of substrate temperature at a pump fluence of $60 \mu \mathrm{Jcm}^{-2}$ (b) for highly doped MEG, sCVDG and pCVDG samples. Carrier relaxation times extracted from fits to theoretical differential $\mathrm{THz}$ transmission $\Delta t / t$ as a function of pump fluence for a few different substrate temperatures (c) and as a function of substrate temperature for a few different pump fluences (d) for highly doped graphene $\left(\varepsilon_{F}=300 \mathrm{meV}\right)$. The theory reproduces the magnitude of the relaxation times and the trends with pump fluence and substrate temperature observed in the experiments. Figure is taken from 107 . 
Chapter 5. Terahertz carrier relaxation dynamics in graphene

\subsubsection{Undoped graphene}

Next, multilayer epitaxial graphene is considered, in which only the first few layers closest to the underlying $\mathrm{SiC}$ substrate have high doping density and the large number of top layers have very low doping density. In these MEG samples, the many top layers with low doping completely dominate the measured $\mathrm{THz}$ carrier dynamics. Figure 5.4 (a,b) shows the representative normalized differential $\mathrm{THz}$ transmission at the peak of the $\mathrm{THz}$ probe pulse, $\Delta t / t$, as a function of pumpprobe delay, for variable pump fluence and for variable substrate temperature, respectively, for an MEG sample with $\sim 63$ layers. In sharp contrast to the high doping case, the differential $\mathrm{THz}$ transmission is negative under all experimental conditions, which corresponds to a pump-induced decrease of the $\mathrm{THz}$ transmission or an increase of the $\mathrm{THz}$ absorption. This observation can be phenomenologically attributed to an increase of the THz conductivity of graphene as the carrier occupation in the conduction band increases upon photoexcitation, which resembles a semiconductor-like behaviour 82, 96. In addition, the $\mathrm{THz}$ carrier dynamics are weakly dependent on the pump fluence, but strongly dependent on the substrate temperature, which is very different to highly doped graphene.

Performing phenomenological fits to the data in Figure 5.4(a,b), the differential $\mathrm{THz}$ transmission evolves from a faster mono-exponential relaxation form at room temperature to a slower bi-exponential relaxation form at cryogenic temperatures. The fast carrier relaxation component results from optical phonon emission. A summary of the extracted carrier relaxation times as a function of pump fluence at room temperature for two MEG samples with $\sim 35$ and $\sim 63$ layers is presented in Figure 5.5(a). The relaxation times at room temperature do not depend on the number of layers, which supports the interpretation that the measured $\mathrm{THz}$ carrier dynamics are dominated by the large number of top layers with low doping. This conclusion is further supported by the fact that the maximum differential $\mathrm{THz}$ transmission signal scales linearly with the number of layers, which is shown in Ref. 107. In addition, the relaxation times of graphene samples with low doping are in the range of $\sim 4-7 \mathrm{ps}$, which is clearly longer than the relaxation times for highly doped graphene samples (see Figure 5.3(a)), because the efficiency of carriercarrier scattering increases with doping density. At very low temperatures, both short and long carrier relaxation times from the bi-exponential fits are extracted, which is associated with two distinct cooling mechanisms. Figure 5.5(b) shows the short relaxation times, which is attributed to carrier-optical-phonon scattering, as a function of substrate temperature for variable pump fluence for the MEG sample with $\sim 63$ layers. The short relaxation times of graphene samples with low doping are weakly dependent on the pump fluence, but strongly dependent on the substrate temperature.

Figure 5.4 (c,d) illustrates the calculated $\mathrm{THz}$ carrier dynamics for undoped 
graphene under similar experimental conditions. The theoretical results reproduce all trends observed in the experiments. As in the high doping case, the hot-carrier relaxation occurs via the interplay between efficient carrier-carrier scattering and carrier-optical-phonon scattering. Figure 5.5(c,d) also show the calculated carrier relaxation times to directly compare with the experiments. In contrast to the highly doped case, the relaxation times decrease significantly with increasing substrate temperature. The impact of substrate-temperature-dependent carrierphonon scattering on the $\mathrm{THz}$ carrier dynamics is negligible. However, the initial Fermi surface, where the THz probe pulse acts on the carriers, strongly depends on the substrate temperature for undoped graphene. Additionally, the fewer thermal carriers are present, the less efficient carrier-carrier scattering is, and this results in longer relaxation times. The calculations also illustrate a weak increase in the relaxation times with increasing pump fluence that can be traced back to hot phonon effects.

At low substrate temperatures, the THz carrier dynamics in the MEG samples with low doping show a long tail on the timescale of hundreds of picoseconds, corresponding to the slow carrier relaxation component in the bi-exponential decay. The $\mathrm{THz}$ carrier dynamics become dependent on the number of layers in the MEG sample, indicating that interlayer thermal coupling effects are important [106]. 

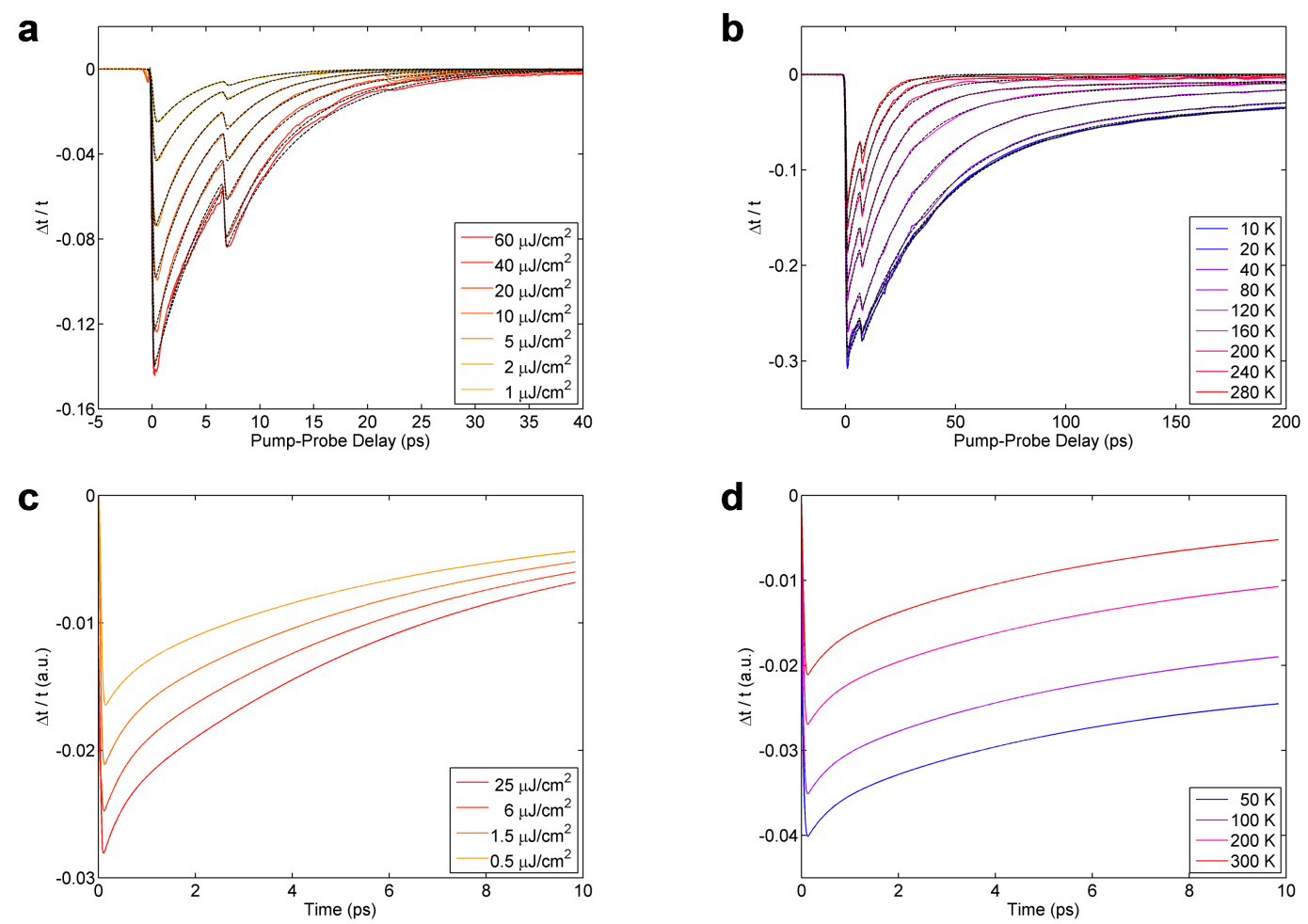

Figure 5.4.: Optical pump and THz probe DTS of graphene with low doping density. Experimental differential THz transmission $\Delta t / t$ at the peak of the $\mathrm{THz}$ probe pulse as a function of pump-probe delay recorded at a substrate temperature of $300 \mathrm{~K}$ for a few different pump fluences (a) and at a pump fluence of $23.4 \mu \mathrm{Jcm}^{-2}$ for a few different substrate temperatures (b) for a lightly doped MEG sample with $\sim 63$ layers. The $\mathrm{THz}$ carrier dynamics evolve from a faster monoexponential relaxation at room temperature to a slower bi-exponential relaxation at cryogenic temperatures. Differential $\mathrm{THz}$ transmission $\Delta t / t$ calculated at a substrate temperature of $300 \mathrm{~K}$ for a few different pump fluences (c) and at a pump fluence of $1.5 \mu \mathrm{Jcm}^{-2}$ for a few different substrate temperatures (d) for undoped graphene. Theory and experiment are in excellent agreement under all conditions. Figure is taken from 107. 

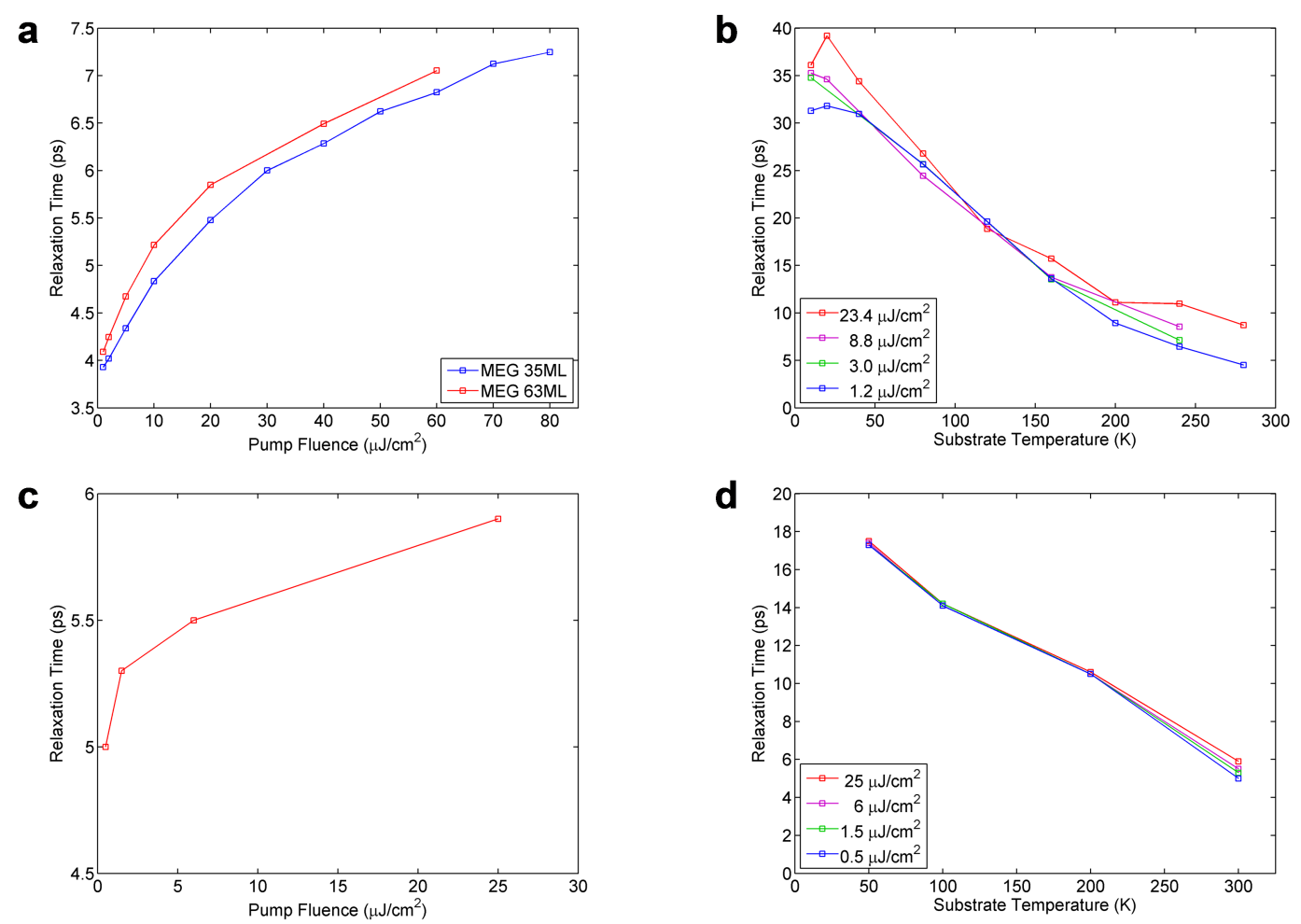

Figure 5.5.: THz carrier relaxation times. Carrier relaxation times extracted from fits to experimental differential $\mathrm{THz}$ transmission $\Delta t / t$ as a function of pump fluence at a substrate temperature of $300 \mathrm{~K}$ (a) and as a function of substrate temperature for a few different pump fluences (b) for lightly doped MEG samples. Carrier relaxation times extracted from fits to theoretical differential $\mathrm{THz}$ transmission $\Delta t / t$ as a function of pump fluence at a substrate temperature of $300 \mathrm{~K}$ (c) and as a function of substrate temperature for a few different pump fluences (d) for undoped graphene. The theory accurately reproduces the magnitude of the relaxation times and the trends with pump fluence and substrate temperature observed in the experiments. Figure is adopted from [107]. 


\subsection{Shortcoming of the Drude model}

To obtain a simple Drude model, the scattering rate is approximated as a constant: $\Gamma_{\lambda \mathbf{k}}^{0}(t)=\Gamma$. The Fourier transform of Eq. 5.4 yields

$$
\delta \rho_{\mathbf{k}}^{\lambda}(\omega)=i \omega \mathbf{e}_{x} \frac{e_{0} A_{0}}{\hbar} \cdot \frac{1}{i \omega-\Gamma} \nabla_{\mathbf{k}} \rho_{\mathbf{k}}^{\lambda, 0} .
$$

The intraband current density is given by:

$$
\mathbf{j}(\omega)=\frac{2 e_{0} \hbar}{i m_{0} L^{2}} \sum_{\mathbf{k}, \lambda} \mathbf{M}_{\mathbf{k}}^{\lambda \lambda} \delta \rho_{\mathbf{k}}^{\lambda}(\omega)
$$

Assuming a Fermi-Dirac distribution for the carrier occupation, the gradient yields

$$
\nabla_{\mathbf{k}} \rho_{\mathbf{k}}^{\lambda}=-\sigma_{\lambda} \mathbf{e}_{\mathbf{k}} \frac{v_{F} \hbar}{4 k_{B} T} \operatorname{sech}^{2}\left[\sigma_{\lambda}\left(k v_{F} \hbar-\varepsilon_{F}\right) / 2 k_{B} T\right] .
$$

For a constant scattering rate $\Gamma$ and with the optical intraband matrix element $\mathbf{M}_{\mathbf{k}}^{\lambda \lambda} \approx i \sigma_{\lambda} M \mathbf{e}_{\mathbf{k}}\left(\sigma_{c}=1\right.$ and $\left.\sigma_{v}=-1\right)$, the intraband current can be evaluated analytically using Eqs. 5.6 and 5.7:

$$
j(\omega)=\frac{e_{0}^{2} M A_{0} k_{B}}{m_{0} \pi c^{2}}\left[i \frac{\Gamma}{\Gamma^{2}+\omega^{2}}-\frac{\omega}{\Gamma^{2}+\omega^{2}}\right] T \ln \left(2+2 \cosh \left(\frac{\varepsilon_{F}}{k_{B} T}\right)\right),
$$

which corresponds to the Drude model. The resulting delay-dependent Drude-like $\mathrm{THz}$ response is given by

$$
\Delta t / t(\tau) \propto j\left(T_{0}, \varepsilon_{F, 0}\right)-j\left(T(\tau), \varepsilon_{F}(\tau)\right),
$$

where $\varepsilon_{F, 0}$ and $T_{0}$ denote the initial Fermi level and carrier temperature before the arrival of the optical pump pulse at $\tau=0$. The time-varying carrier temperature $T(\tau)$ and quasi-Fermi level $\varepsilon_{F}(\tau)$ are formed after the thermalization of the pump-induced non-equilibrium carrier population. The Drude-like differential $\mathrm{THz}$ transmission $\Delta t / t$ (Eq. 5.10 calculated for highly doped graphene $\left(\varepsilon_{F}=300 \mathrm{meV}\right)$ is mapped in Figure 5.6(a). It is apparent (dashed line) that if the effect of the optical pump pulse was only to heat the carriers without changing the Fermi level $\left(\varepsilon_{F}=\varepsilon_{F, 0}\right)$, then a negative sign of the $\Delta t / t$ signal would always be observed. For a constant $\Gamma_{\lambda, \mathbf{k}}^{0}(t)=\Gamma$, carrier heating alone cannot explain the positive sign of the differential $\mathrm{THz}$ transmission observed in highly doped graphene.

Figure 5.6(a) also illustrates the pump-induced temporal evolution of $T(\tau)$ and $\varepsilon_{F}(\tau)$ obtained by solving the full graphene Bloch equations within this approximation for low and high excitation $\left(12.5 \mu \mathrm{Jcm}^{-2}\right.$ (red line) and $80 \mu \mathrm{Jcm}^{-2}$ (blue line)). Interestingly, not only carrier heating is found, but also a transient decrease of the 
Chapter 5. Terahertz carrier relaxation dynamics in graphene

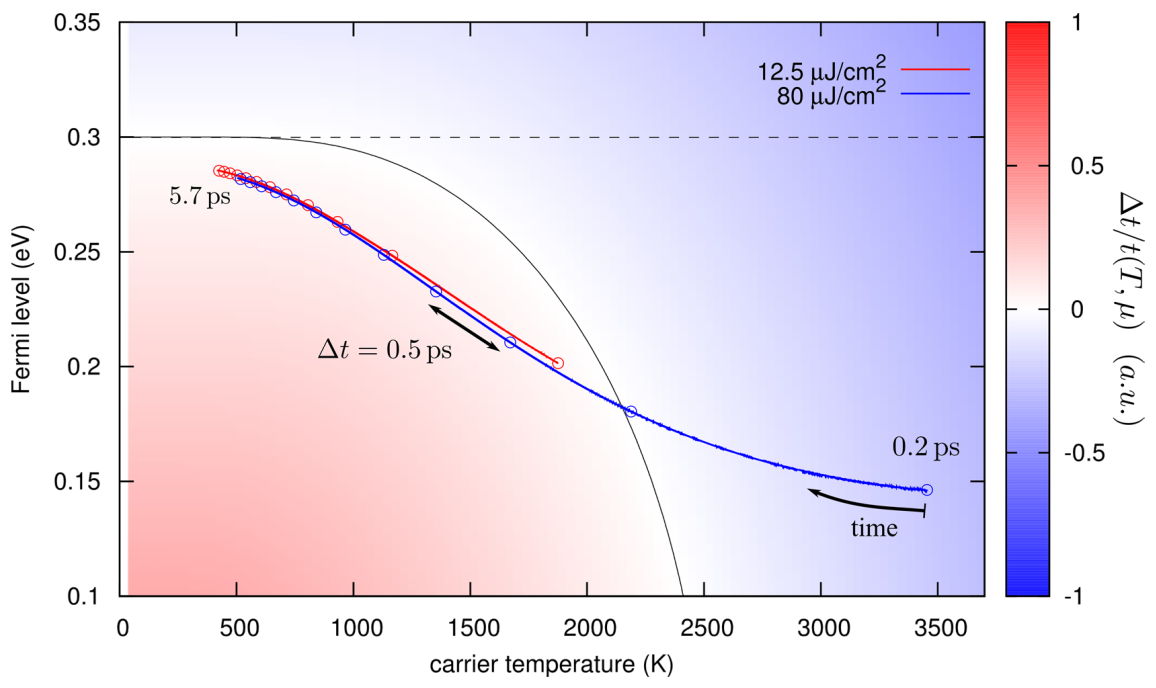

Figure 5.6.: Microscopic theory calculation of carrier dynamics and carrier scattering time. Differential THz transmission assuming a Drude model for an initial temperature of $300 \mathrm{~K}$ and a Fermi level of $300 \mathrm{meV}$ as a function of the transient carrier temperature $T$ and Fermi level $\varepsilon_{F}$. The dashed line illustrates the possible differential $\mathrm{THz}$ transmission for a constant Fermi level. The solid black line separates regions of positive and negative $\Delta t / t$. The red (blue) line shows the path through the $T-\varepsilon_{F}$-map for a pump fluence of $12.5 \mu \mathrm{Jcm}^{-2}\left(80 \mu \mathrm{Jcm}^{-2}\right)$ obtained from the solution of the full graphene Bloch equations for the pumpinduced dynamics. The first point reflects the system $0.2 \mathrm{ps}$ after the pump pulse, when the carrier distribution can be extrapolated by a Fermi distribution. The time delay between two points is $0.5 \mathrm{ps}$, respectively.

quasi-Fermi level which reduces the THz absorption, as implied by Eq. 5.9. Obviously, the pump-induced shift of the Fermi level outweighs the impact of carrier heating. As a consequence, a positive sign of the $\Delta t / t$ signal for the lower pump fluence (red line) at zero time delay and for the higher pump fluence (blue line) after $0.8 \mathrm{ps}$ if found. Thus, the positive $\Delta t / t$ signal for highly doped graphene observed in the measurements can be explained to large extent by a standard Drude model with constant $\Gamma$, provided that the carrier heating and cooling dynamics as well as the Fermi level dynamics are described correctly. Previously, the observation of a positive differential $\mathrm{THz}$ transmission in doped graphene has been attributed phenomenologically to a pump-induced increase in the carrier scattering rate, and a corresponding decrease of the THz conductivity within a Drude model [98-100], which is essentially a metal-like behaviour, although the mechanism responsible for the increase in the carrier scattering rate could not be positively identified. Here, 
the positive $\Delta t / t$ signal can be partly explained within a simple Drude picture as a consequence of the Fermi level shift. However, the simple Drude model is not sufficient to explain the behaviour at higher pump fluence, for which the short time dynamics would exhibit a negative $\Delta t / t$ signal, unless other phenomenological parameters such as a carrier heating efficiency [101,102 or a non-monotonic carrier-temperature-dependent Drude weight are included |103|.

A complete understanding is obtained by applying the full microscopic formalism, Eqs. (5.4) and 4.11), including the explicitly time- and momentumdependent scattering rates $\Gamma_{\lambda, \mathbf{k}}^{0}(t)$ of the $\mathrm{THz}$ probe-induced carrier dynamics. The results are shown in Figure $5.2(\mathrm{c}, \mathrm{d})$, exhibiting excellent agreement with the experiment, for low as well as high pump fluence. Specifically, in both experiment and theory, an overall positive differential $\mathrm{THz}$ transmission is found at all time delays and all pump fluences. Thus, the microscopic model for the pump-induced carrier scattering is essential to capture the very initial THz carrier dynamics correctly. In the regime of high fluence and short time, even if carrier thermalization is complete, a constant carrier scattering rate approximation cannot be trusted. In particular, in the strong excitation regime the efficient time-dependent Coulomb scattering provides the major contribution. In principle a $\mathbf{k}$-dependent $\Gamma_{\mathbf{k}}$ could also be considered in Eqs. 5.6 and 5.7 to obtain a more advanced Drude model. However, this would require also an approximate analytical model for $\Gamma_{\mathbf{k}}$.

An important question in the hot-carrier dynamics is the possible energy dependence of the carrier scattering rate (inverse time), and the consequences for the $\mathrm{THz}$ carrier dynamics. The microscopic theory calculations performed here give further insight into this point, where the carrier scattering rate as the exponential decay of the carrier occupation is considered. 
Calculation of the carrier scattering time in graphene Next, a relation between the microscopic scattering rates and the exponential decay of the carrier occupation will be derived. The collision part of the Boltzmann equation, which is given by

$$
\frac{d}{d t} \rho_{\mathbf{k}}^{\lambda}=\Gamma_{\lambda, \mathbf{k}}^{\mathrm{in}}\left[1-\rho_{\mathbf{k}}^{\lambda}\right]-\Gamma_{\lambda, \mathbf{k}}^{\text {out }} \rho_{\mathbf{k}}^{\lambda}
$$

can be rewritten as:

$$
\frac{d}{d t} \rho_{\mathbf{k}}^{\lambda}=-\frac{\rho_{\mathbf{k}}^{\lambda}-\tau_{\lambda, \mathbf{k}} \Gamma_{\lambda, \mathbf{k}}^{\mathrm{in}}}{\tau_{\lambda, \mathbf{k}}},
$$

with

$$
\tau_{\lambda, \mathbf{k}}:=\frac{1}{\Gamma_{\lambda, \mathbf{k}}^{\mathrm{in}}+\Gamma_{\lambda, \mathbf{k}}^{\mathrm{out}}} .
$$

The term $\tau_{\lambda, \mathbf{k}} \Gamma_{\lambda, \mathbf{k}}^{\text {in }}$ can be written as:

$$
\tau_{\lambda, \mathbf{k}} \Gamma_{\lambda, \mathbf{k}}^{\text {in }}=\frac{\Gamma_{\lambda, \mathbf{k}}^{\text {in }}}{\Gamma_{\lambda, \mathbf{k}}^{\text {in }}}+\Gamma_{\lambda, \mathbf{k}}^{\text {out }}=\frac{1}{1+\frac{\Gamma_{\lambda, \mathbf{k}}^{\text {out }}}{\Gamma_{\lambda, \mathbf{k}}^{\text {in }}}} .
$$

Accounting only for weak excitations, the scattering rates can be approximated with the equilibrium rates $\Gamma_{\lambda, \mathbf{k}}^{\mathrm{in}, \text { out }, 0}$ fulfilling the principle of detailed balance (D.B.):

$$
\frac{\Gamma_{\lambda, \mathbf{k}}^{\text {out }}}{\Gamma_{\lambda, \mathbf{k}}^{\text {in }}} \approx \frac{\Gamma_{\lambda, \mathbf{k}}^{\text {out }, 0}}{\Gamma_{\lambda, \mathbf{k}}^{\text {in }, 0}} \stackrel{\text { D.B. }}{=} e^{\left(\varepsilon_{k}-\varepsilon_{F}\right) / k_{B} T} .
$$

Thus, Eq. 5.14 represents the initial Fermi distribution $\rho_{\mathbf{k}}^{\lambda, 0} \approx \tau_{\lambda, \mathbf{k}} \Gamma_{\lambda, \mathbf{k}}^{\text {in }}$ and the Boltzmann equation yields the relaxation-time model

$$
\frac{d}{d t} \rho_{\mathbf{k}}^{\lambda}=-\frac{\rho_{\mathbf{k}}^{\lambda}-\rho_{\mathbf{k}}^{\lambda, 0}}{\tau_{\lambda, \mathbf{k}}}
$$

Within the approximation in Eq. 5.15. Eq. 5.16 clearly reveals a direct relation between the scattering rates and the exponential decay of the carrier population. However, by fitting the numerical data stemming from the full scattering equation (Eq. 5.11), the method for the determination of the carrier scattering time (see Figure 5.7) goes beyond the relaxation-time approximation.

By fitting the numerically calculated hot-carrier dynamics, the energy relaxation time $\tau(\epsilon)$ of the photoexcited carriers is obtained. Figure 5.7 shows the calculated carrier scattering time $\tau(\epsilon)$ as a function of carrier energy $\epsilon$ for pristine graphene at a substrate temperature of $300 \mathrm{~K}$. The carrier scattering time is precisely inverse to the carrier energy, $\tau(\epsilon)=\beta /|\epsilon|$ (with $\beta \approx 0.9 \mathrm{eVps}$ ), over a very broad energy range $(|\epsilon| \sim 0.2-1.5 \mathrm{eV})$ in agreement with previous experimental studies on 
Chapter 5. Terahertz carrier relaxation dynamics in graphene

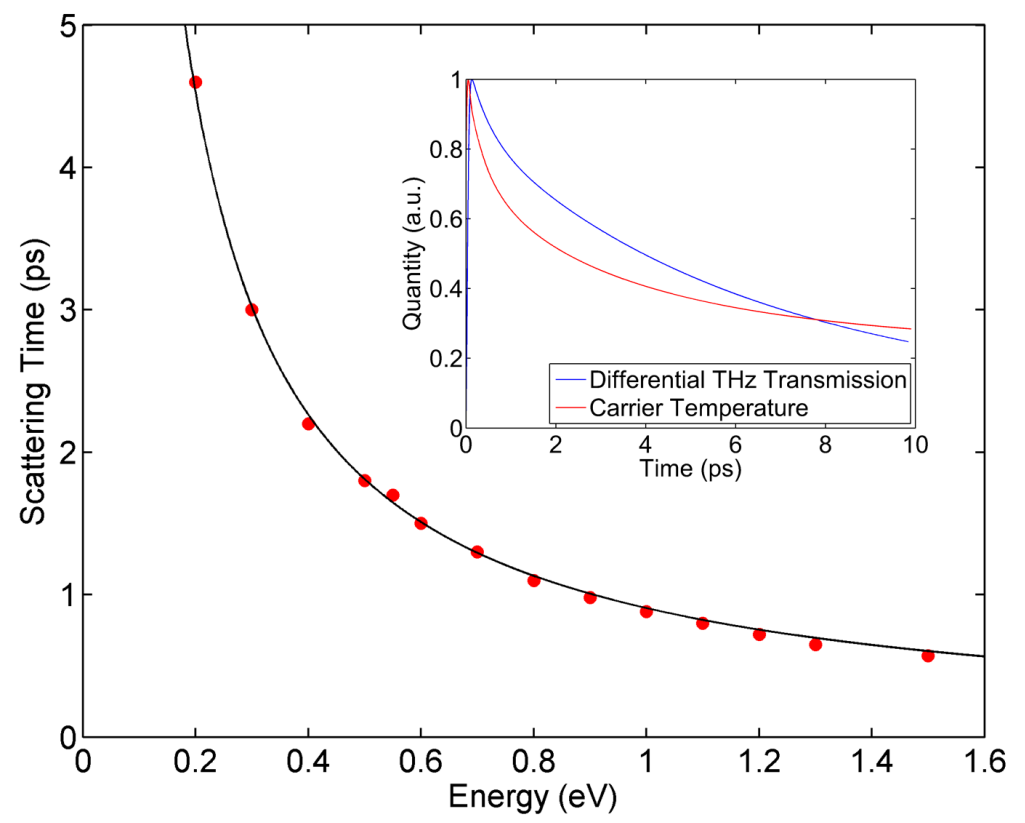

Figure 5.7.: Carrier scattering time $\tau(\epsilon)$ as a function of carrier energy $\epsilon$ calculated for undoped graphene at a temperature of $300 \mathrm{~K}$. The calculated values are inverse to the carrier energy, $\tau(\epsilon)=\beta /|\epsilon|$ (with $\beta \approx 0.9 \mathrm{eV}$ ps). The inset shows a comparison between the normalized differential THz transmission and the normalized carrier temperature dynamics calculated for undoped graphene at a substrate temperature of $300 \mathrm{~K}$ and a pump fluence of $1.5 \mu \mathrm{Jcm}^{-2}$. The two dynamics relax on similar, but not exactly equal timescales.

MEG 23 and graphite 120, 121. These results are in contrast with the linear relation on carrier energy, $\tau(\epsilon)=\alpha|\epsilon|$, 122,123 that is inferred from electrical transport measurements in some graphene samples. This is attributed to the fact that in electrical transport measurements carriers have energies close to the Fermi energy and carrier scattering in these graphene samples is dominated by extrinsic mechanisms such as defects, charge impurities, wrinkles and ripples 124, 125.

Finally, the microscopic theory allows to directly address another important question in ultrafast time-resolved $\mathrm{THz}$ spectroscopy: To what extent does the differential $\mathrm{THz}$ transmission measure the carrier temperature dynamics? The transient differential $\mathrm{THz}$ transmission and transient carrier temperature are not directly proportional to each other, but are connected through the macroscopic current density. The inset of Figure 5.7 shows a direct comparison between the differential $\mathrm{THz}$ transmission and the carrier temperature dynamics for undoped graphene at a substrate temperature of $300 \mathrm{~K}$ and a pump fluence of $1.5 \mu \mathrm{Jcm}^{-2}$. 
It can be clearly seen, that the differential $\mathrm{THz}$ transmission does not exactly follow the carrier temperature dynamics, which reveals that both transient carrier temperature and Fermi level shifts are essential to capture the dynamic $\mathrm{THz}$ response correctly. In particular, the relaxation times extracted from the decay of the differential $\mathrm{THz}$ transmission are in general not exactly equal to the electronic cooling times, although they may serve as a useful approximation as the two dynamics relax on similar timescales. 


\section{Impact of doping on carrier relaxation dynamics in graphene}

In the last years a number of theoretical and experimental studies were performed to obtain a deep understanding of the carrier relaxation dynamics in graphene $17,20,23,25,29,56,126,127$. Most of these studies focus on the ultrafast Coulomb- and phonon-induced carrier dynamics without considering the influence of the doping in the investigated graphene samples. However, an extrinsic carrier density can have a crucial impact on the relaxation dynamics via a significant increase in the scattering phase space and the enhanced effect of Pauli blocking, which causes both the carrier dynamics and consequently also the performance of graphene in opto-eletronic devices.

A first experimental time-resolved ARPES study has been performed addressing the doping dependence of carrier multiplication of graphene $|128|$. The underlying elementary processes determining the observed different behavior for $\mathrm{p}$ - and n-doped samples have not been understood, yet. The main part of this chapter lies in particular on the impact of doping on the appearance of the technologically relevant carrier multiplication (CM) $30,32,33,101,128,135]$. This is an interesting ultrafast phenomenon that is related to the linear electronic band structure of graphene opening up the possibility of efficient Coulomb-induced Auger processes. A significant $\mathrm{CM}$ has been theoretically predicted $30,130,134$ and experimentally confirmed in graphene [32, 101, 128, 133, 135. So far, the theoretical studies have been constrained to the case of undoped graphene. Introducing doping, one has to distinguish between the Coulomb-induced processes of carrier multiplication (CM) and hot carrier multiplication (hCM), cf. Fig. 6.5. Auger scattering bridging the valence and the conduction band gives rise to $\mathrm{CM}$ corresponding to an increase of the number of charge carriers in graphene. In contrast, $\mathrm{hCM}$ is induced by Coulomb-induced intraband scattering bridging the states below and above the Fermi level and resulting in an increase of the number of hot carriers. Here, the actual number of charge carriers remains unchanged in each band. Nevertheless, since these hot carriers are crucial for many technological applications, the appearance of hCM is also of technological relevance.

Another interesting study is the orientational relaxation in graphene. Since the optical matrix element shows an anisotropy in the momentum space the optically excited non-equilibrium is highly anisotropic at the beginning 29, 136. It could be 
Chapter 6. Impact of doping on carrier relaxation dynamics in graphene

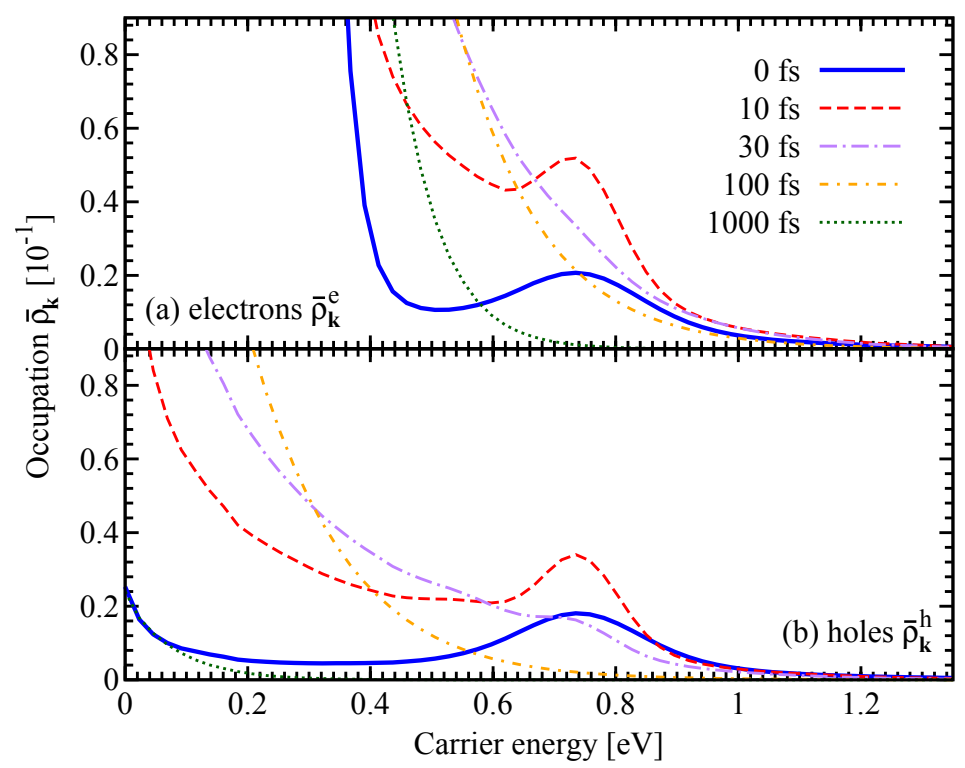

Figure 6.1.: Angle-averaged occupation probability $\bar{\rho}_{\mathbf{k}}$ for (a) electrons and (b) holes in highly $\mathrm{n}$-doped graphene $\left(\varepsilon_{f}=300 \mathrm{meV}\right)$ is shown as a function of the carrier energy for different times after the optical excitation. Note that due to the n-doping the electron relaxation is faster resulting in a thermalized hot Fermi distribution already after 30 fs.

shown, that the initially highly anisotropic carrier occupation becomes efficiently redistributed due to electron-phonon and electron-electron scattering processes within few tens of femtosecond [136].

In this chapter the microscopic theory is applied to investigate the relaxation dynamics of optically excited carriers in doped graphene. Being able to track the carrier resolved in time, angle and momentum and to apply precisely the same excitation conditions for pristine and doped graphene, the impact of doping on the carrier dynamics, anisotropic excitation, and carrier multiplication are systematically worked out.

\subsection{Carrier occupation and polarization}

In this section, the carrier dynamics in highly doped graphene is studied, in particular the impact of doping on the carrier occupation and microscopic polarization is studied. Furthermore, the influence of the non-diagonal dephasing and the screening model on polarization is presented.

Figure 6.1 illustrates the the angle-averaged occupation probability $\bar{\rho}_{\mathbf{k}}^{\lambda}$ for (a) elec- 
Chapter 6. Impact of doping on carrier relaxation dynamics in graphene

trons and (b) holes for an initial n-doping of $300 \mathrm{meV}$ as a function of the carrier energy for different times after the optical excitation. Note that for symmetry reasons, the physical picture remains the same for holes in p-doped graphene samples. The system is excited by an $10 \mathrm{fs}$ pulse at an energy of $1.5 \mathrm{eV}$ with a pump fluence of $0.3 \mu \mathrm{Jcm}^{-2}$. The characteristics of the excitation pulse correspond to typical values that can be realized by standard pulsed lasers $[22$. The pulse is centered at $0 \mathrm{fs}$ and gives rise to a well pronounced non-equilibrium distributions for electrons and holes around the carrier energy of $0.75 \mathrm{eV}$ (corresponding to the spectral position of the excitation pulse), cf. Fig. 6.1 (a) and (b). For the n-doped sample, the electronic states are fully occupied up to the Fermi energy of $300 \mathrm{meV}$. For both electrons and holes, the efficient carrier-carrier and carrier-phonon scattering leads to an ultrafast thermalization of the system towards a hot Fermi distribution already after some tens of femtoseconds. Then, a slower phonon-induced carrier cooling occurs that drives the electron and hole occupations towards their initial thermal Fermi distributions. Due to the increased number of available scattering partners in the conduction band of n-doped graphene, the Coulomb-driven carrier thermalization occurs faster for electrons. Here, a hot thermalized Fermi distribution is already reached after $30 \mathrm{fs}$, cf. the purple line in Fig. 6.1(a).

Now, the impact of doping on the imaginary part of the microscopic polarization $p_{\mathbf{k}_{0}}(t)$ is discussed. with the excitation momentum $k_{0}=\hbar \omega_{L} / 2 v_{F}$, cf. Fig. 6.2 (a). The doped case shows a weaker polarization compared to the undoped case. In doped graphene the many-particle dephasing $\gamma_{\mathbf{k}}$ (cf. Eq. 2.72), which is determined scattering rates $\Gamma_{\mathbf{k} \lambda}^{\text {in,out }}$, is considerably larger leading to a more efficient dephasing. As a result, the strong dephasing in the doped case leads to a faster decay of the polarization. The calculations also reveal that the off-diagonal contribution $\mathcal{U}_{\mathbf{k}}$ of Eq. (2.72) and the screening model play an important role for the polarization dynamics. Accounting only the diagonal dephasing, as shown in Fig. 6.2 (b) (red line), it can be seen that the excited oscillations decay within few femtoseconds. This can be seen from the underlying equation of motion for the polarization (Eq. 2.72, where the diagonal dephasing appears as an exponential damping $\gamma_{\mathbf{k}}$ constituted by the sum of the scattering rates. In contrast to the diagonal case, the off-diagonal dephasing (blue line) couples the polarization dynamics at different quantum numbers $\mathbf{k}^{\prime}$, which gives rise to an enhanced coherence at $k_{0}$, cf. Eq. (2.74). With the contribution of the off-diagonal dephasing the microscopic polarization directly couples to the carrier dynamics during the excitation pulse and this results in the generation of a higher nonequilibrium distribution [41]. For higher pump fluences, this effect becomes even more crucial. The diagonal dephasing decreases the transition in a certain state $\mathbf{k}$ and the full dephasing increases the probability of the state $\mathbf{k}[89$.

Figure 6.2 (c) illustrate the difference between the static (red line) and dynamical screening (blue line) on the polarization. The calculations reveal that the dynam- 
Chapter 6. Impact of doping on carrier relaxation dynamics in graphene

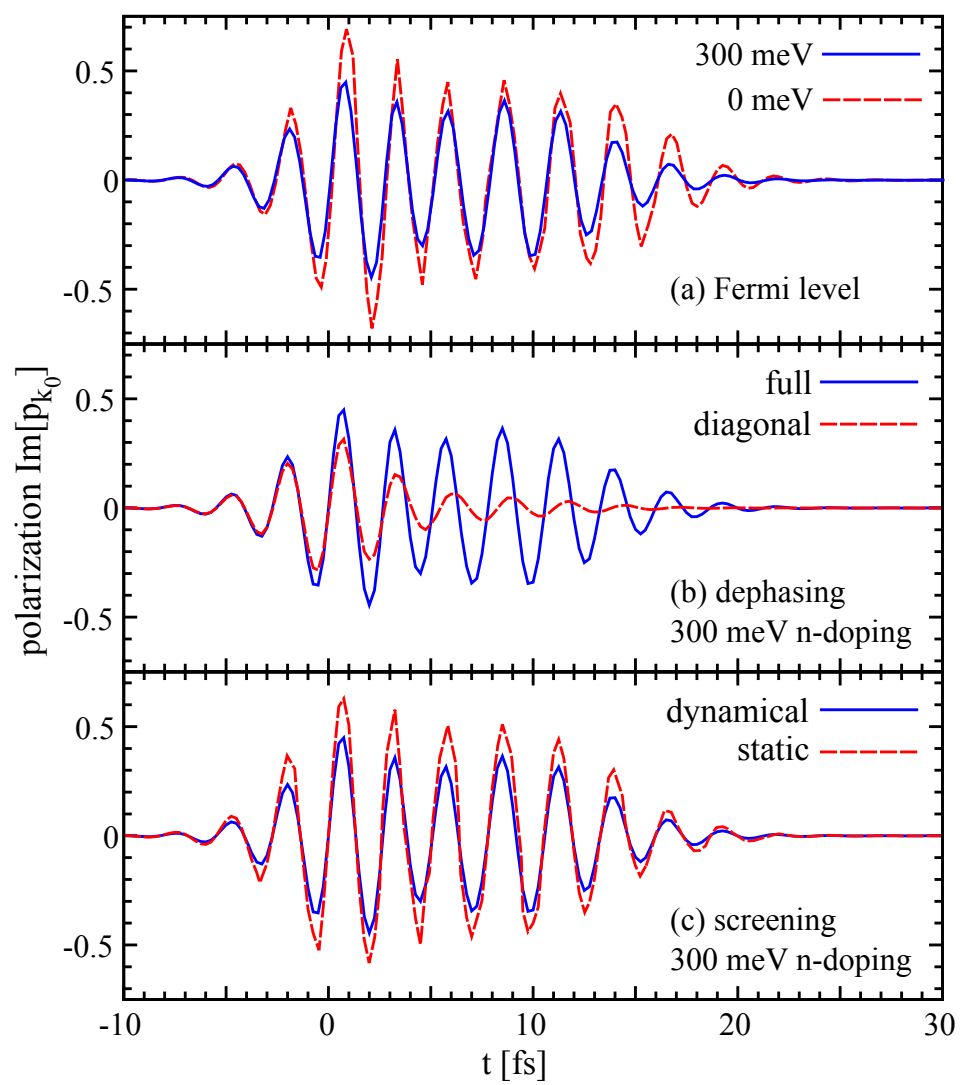

Figure 6.2.: (a) The influence of doping on the imaginary part of the microscopic polarization $p_{\mathbf{k}_{0}}$ at the excitation $k_{0}$. (b) Imaginary part of the microscopic polarization at the excitation momentum $k_{0}$ for n-doped graphene $\left(\varepsilon_{f}=300 \mathrm{meV}\right)$ with and without the contribution of the off-diagonal many-particle dephasing. (c) The influence of the screening model on the microscopic polarization for n-doped graphene.

ical screening is considerably stronger compared to the static limit leading to a weaker polarization.

\subsection{Orientational carrier relaxation}

In this section the anisotropy of the initially excited distribution in highly doped is presented, where the carrier orientation $\phi_{\mathbf{k}}$ of the two-dimensional momentum space $\mathbf{k}=\left(k, \phi_{\mathbf{k}}\right)$ is considered. Taking realistic parameters from experiments, the system is excited by an $10 \mathrm{fs}$ pulse at $\hbar \omega_{L}=1.5 \mathrm{eV}$ and a pump fluence of $2.3 \mu \mathrm{Jcm}^{-2}$ [22. Figure 6.3 shows the fully two-dimensional resolved electron dis- 
Chapter 6. Impact of doping on carrier relaxation dynamics in graphene

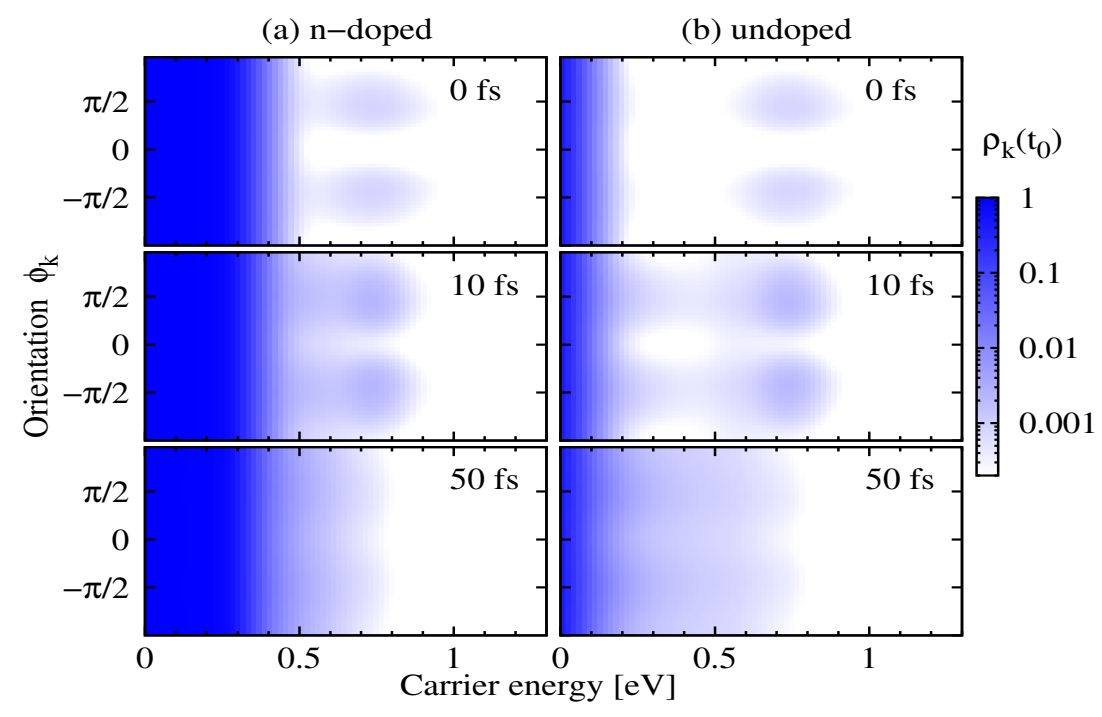

Figure 6.3.: Fully momentum resolved carrier occupation $\rho_{k, \phi}$ as a function of the momentum $\mathrm{k}$ and the orientation angle $\phi$ with respect to the Dirac point for (a) highly doped and (b) undoped graphene for selected times after the pulse maximum. The initially highly anisotropic carrier distribution equilibrates to a large extent due to the efficient carrier-phonon scattering processes within $50 \mathrm{fs}$.

tribution $\rho_{k, \phi}^{c}(t)$ of n-doped with a doping level of $300 \mathrm{meV}$ (a) and undoped (b) graphene, where the angle $\phi$ describes the carrier orientation with respect to the excitation pulse and the momentum $\mathrm{k}$. In undoped graphene, the initially highly anisotropic carrier distribution equilibrates due to the efficient carrier-carrier and carrier-phonon scattering processes on a $100 \mathrm{fs}$ timescale [28, 29], cf. Fig. 6.3 [28]. The maximal occupation is found perpendicular with respect to the pump pulse polarization, in particular at the carrier energy $0.75 \mathrm{eV}$ and the orientation angle $\phi= \pm \pi / 2$. The anisotropy of the carrier-light interaction gives rise to a preferred carrier excitation [41. It can be shown, that scattering with optical phonons provides highly efficient orientational relaxation, whereas Coulomb scattering is mainly responsible for an ultrafast momentum redistribution [28]. In doped graphene, practically no difference in the relaxation dynamics is seen, since the time to reach an isotropic carrier distribution is almost the same, cf. Fig. 6.3. a). The reason is the following: optical phonon scattering is mainly responsible for the orientational relaxation and since they are relatively insensitive to the carrier density, the additional extrinsic carriers has no major impact on the orientational relaxation [95. As a result, doping does not play a significant role for the orientational relaxation dynamics as long as the excitation energy is significantly larger than the Fermi level. 


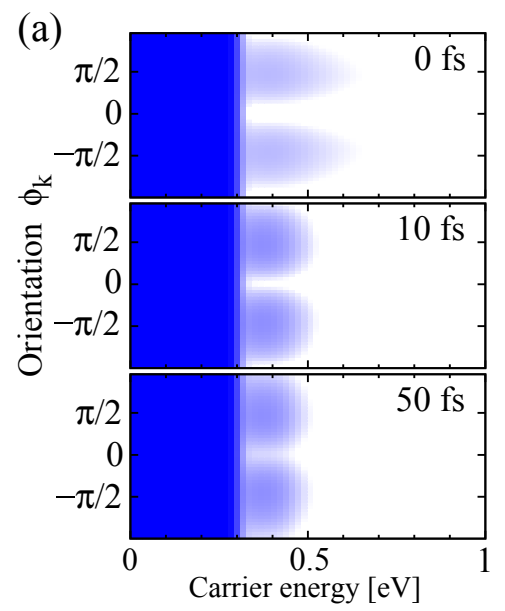

(b)

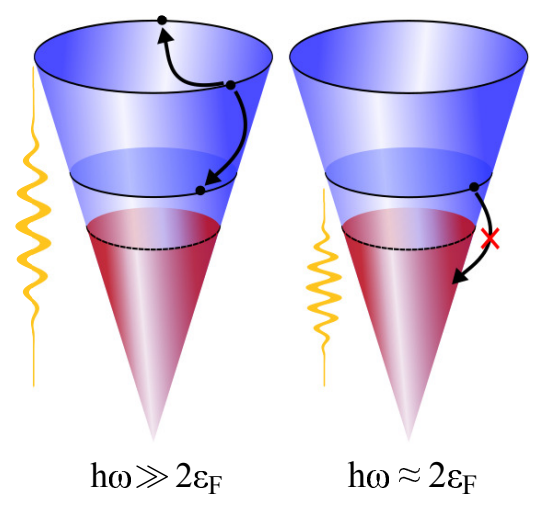

Figure 6.4.: (a) Carrier occupation $\rho_{k, \phi_{k}}$ as a function of the momentum $\mathrm{k}$ and the orientation angle $\phi$ around the Dirac point for highly doped graphene with an excitation near the Fermi level. The non-equilibrium distribution still remains anisotropic after 50 fs. (b) Illustration of phonon-induced scattering processes with an excitation energy considerably larger than the Fermi level and near the Fermi level. In the latter case, carrier-phonon scattering processes are almost suppressed due to the strict Markovian energy conservation.

Next, the relaxation dynamic is studied by exciting the system sligthly above the Fermi level with $0.75 \mathrm{eV}$, which is illustrated in Figure 6.4(a). With an excitation energy near the Fermi level the non-equilibrium still remains at $50 \mathrm{fs}$. In this case, carrier-phonon scattering processes due to the large phonon energies are almost suppressed by Pauli-Blocking in the final carrier lying below the Fermi level. In particular, the emission of phonons is suppressed due to the strict Markovian energy conservation, since the constant dispersion energies of the optical phonons between 160 and $200 \mathrm{meV}$ are too large for efficient scattering processes, cf. Fig. 6.4(b). As a result, the orientational relaxation dynamics is significantly slower. The contribution of acoustic phonons is negligibly small for the first tens of femtoseconds [41. 


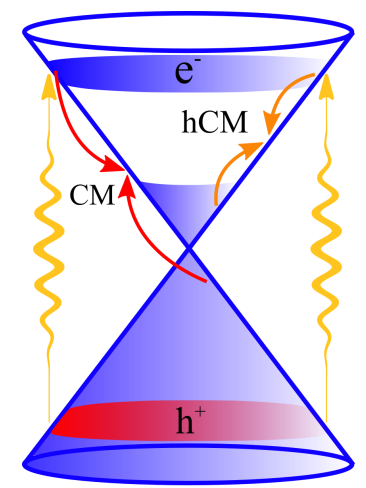

Figure 6.5.: Schematic illustration of the impact excitation in the linear regime of the electronic band structure for n-doped graphene with the optically excited hole $\rho_{\mathbf{k}}^{h}$ and electron occupation probabilities $\rho_{\mathbf{k}}^{e}$. In doped graphene, the technologically relevant many-particle processes of carrier multiplication (CM) and hot carrier multiplication (hCM) need to be distinguished. While the first are induced by Auger processes bridging the valence and the conduction band (red arrows) increasing the number of charge carriers in the system, the second corresponds to Coulomb-induced intraband scattering (orange arrows) increasing the number of hot (high-energy) carriers. Figure is taken from [137|.

\subsection{Carrier Multiplication in doped graphene}

An interesting ultrafast phenomenon related to the linear electronic band structure of graphene is the possibility of efficient Coulomb-induced Auger processes bridging the valence and the conduction band and changing the number of charge carriers in the system. A significant carrier multiplication has been theoretically predicted [30,130,134 and experimentally confirmed in graphene [32,101, 128, 133, 135]. So far, the theoretical studies have been constrained to the case of undoped graphene. Introducing doping, one has to distinguish between the Coulomb-induced processes of carrier multiplication (CM) and hot carrier multiplication (hCM), cf. Fig. 6.5. Auger scattering bridging the valence and the conduction band gives rise to $\mathrm{CM}$ corresponding to an increase of the number of charge carriers in graphene. In contrast, hCM is induced by Coulomb-induced intraband scattering bridging the states below and above the Fermi level and resulting in an increase of the number of hot carriers. Here, the actual number of charge carriers remains unchanged in each band. Nevertheless, since these energetically high hot carriers are crucial for many optoelectronic applications, the appearance of $\mathrm{hCM}$ is of technological relevance.

In this section, the impact of doping on Auger scattering channels and the 

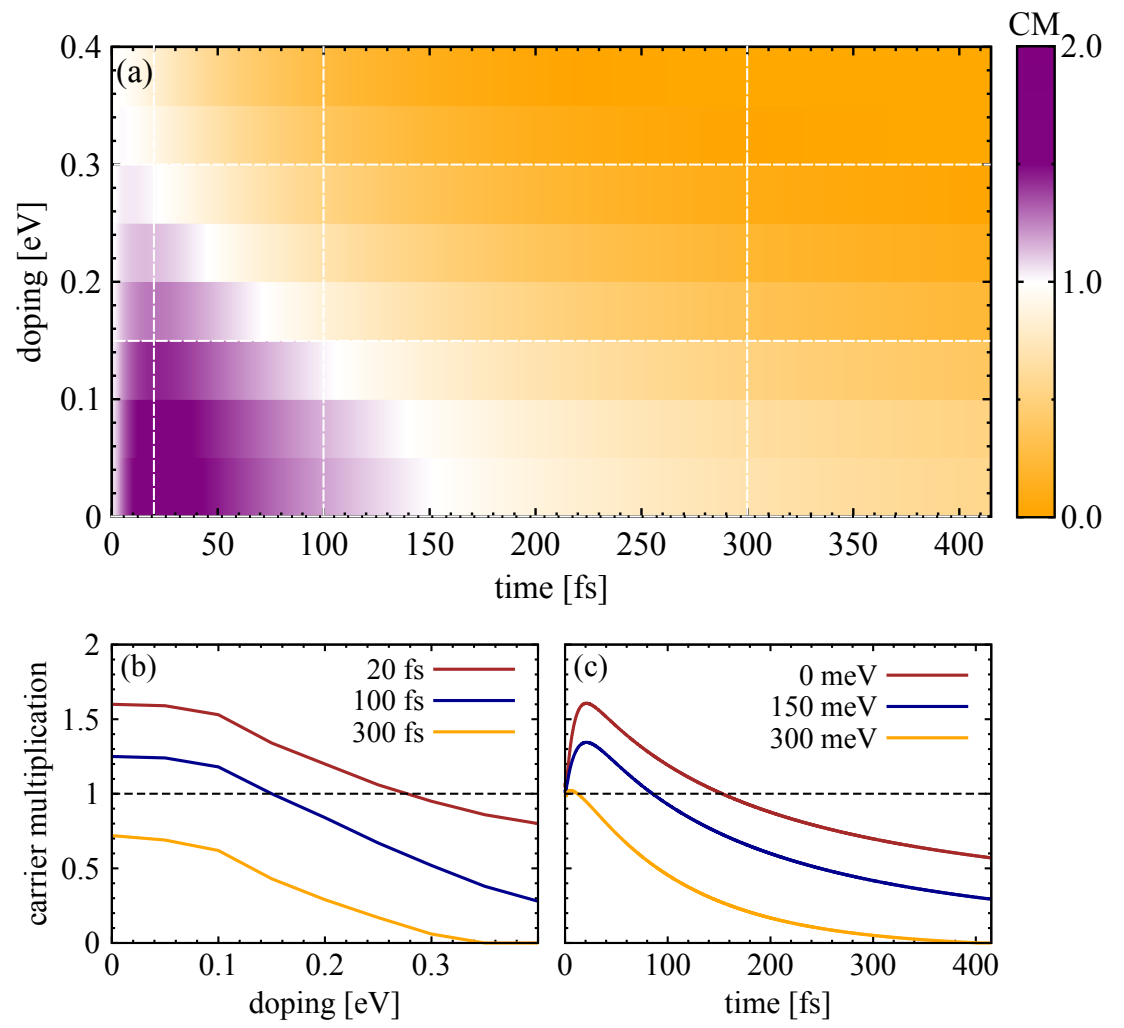

Figure 6.6.: (a) Temporal evolution of the doping-dependent carrier multiplica-

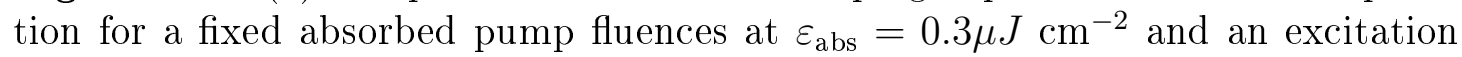
energy of $1.5 \mathrm{eV}$. (b) Doping-dependent CM for three fixed time delays and (c) the temporal evolution of CM for different doping levels. Figure is taken from [137].

appearance of carrier multiplication in realistic doped graphene samples is studied. Here, one has to distinguish between the carrier generating impact excitation (IE) and its inverse process, the Auger recombination (AR). In distinct excitation regimes, the IE has a higher probability than AR leading to a theoretically predicted and experimentally confirmed carrier multiplication (CM) 30, 128, 133, 135]. $\mathrm{CM}$ is generally defined as the ratio between the number of overall generated electron-hole pairs and the optically excited charge carriers:

$$
\mathrm{CM}_{\text {generally }}=\frac{n-n_{T}}{n_{\text {opt }}}
$$

where $n$ is the total carrier density, $n_{T}$ the initial thermal carrier background, and $n_{\text {opt }}$ the optically excited carrier density. All contributions contain both electrons $e$ in the conduction band as well as holes $h$ in the valence band. For doped graphene, the specific definition depends on the physical situation: For optical measurements 
Chapter 6. Impact of doping on carrier relaxation dynamics in graphene

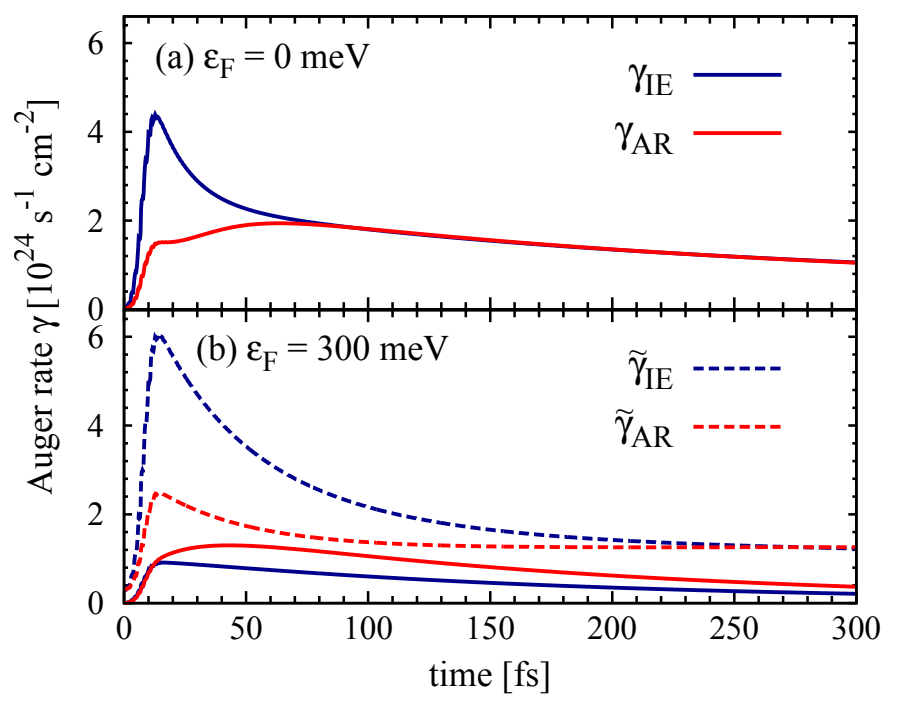

Figure 6.7.: Temporal evolution of Auger rates including impact excitation (IE) and the inverse process of Auger recombination (AR) for (a) undoped and (b) highly n-doped graphene $(\varepsilon=300 \mathrm{meV})$. In the latter case, also the corresponding 'intraband Auger rates' describing Coulomb-induced scattering bridging the states below and above the Fermi level are illustrated (dashed lines), cf. Fig. 6.5. In undoped graphene, there is a clear asymmetry between both Auger processes in favor of IE in the first $100 \mathrm{fs}$. In the doped case, the behavior is inverse and $\gamma_{A R}$ is significantly larger than $\gamma_{I E}$ for a time period longer than $300 \mathrm{fs}$. For 'intraband Auger rates', the situation is different and $\tilde{\gamma}_{I E}$ clearly prevails over $\tilde{\gamma}_{A R}$. Figure is adopted from 137.

probing vertical carrier transitions, a definition with respect to to the Dirac point is reasonable, i.e. $\rho_{\mathbf{k}}^{e}=\rho_{\mathbf{k}}^{c}$ and $\rho_{\mathbf{k}}^{h}=1-\rho_{\mathbf{k}}^{v}$. The carrier density then reads

$$
n_{\mathrm{CM}}\left(T, \varepsilon_{F}\right)=\frac{\sigma_{s} \sigma_{v}}{L^{2}} \sum_{\lambda=e, h ; \mathbf{k}} \rho_{\mathbf{k}}^{\lambda}
$$

where $L^{2}$ is the graphene area and $\sigma_{s}\left(\sigma_{v}\right)$ denotes the spin (valley) degeneracy. On the other side, electric transport phenomena are also of great interest, where hot electrons around the Fermi level are relevant. In this situation, charge carriers are defined with respect to the Fermi level, i.e. for n-doped graphene the upper Dirac cone is splited into $\rho_{\mathbf{k}}^{h, c}=1-\rho_{\mathbf{k}}^{c}$ for $k<k_{F}$ and $\rho_{\mathbf{k}}^{e, c}=\rho_{\mathbf{k}}^{c}$ for $k>k_{F}$ with the Fermi momentum $k_{F}$. The bottom cone remains unaffected with $\rho_{\mathbf{k}}^{h, v} \equiv \rho_{\mathbf{k}}^{h}=1-\rho_{\mathbf{k}}^{v}$. The 
Chapter 6. Impact of doping on carrier relaxation dynamics in graphene

according hot carrier density $n_{\mathrm{hCM}}$ is given by

$$
n_{\mathrm{hCM}}\left(T, \varepsilon_{F}\right)=\frac{\sigma_{s} \sigma_{v}}{L^{2}}\left(\sum_{\mathbf{k}} \rho_{\mathbf{k}}^{h}+\sum_{\mathbf{k}}^{k<k_{F}} \rho_{\mathbf{k}}^{h, c}+\sum_{\mathbf{k}}^{k>k_{F}} \rho_{\mathbf{k}}^{e, c}\right),
$$

and the associated carrier multiplication for transport phenomena is labeled as hot carrier multiplication (hCM) [101, 128], cf. Fig. 6.5. Note that symmetric results are obtained for n- and p-doped graphene. For undoped graphene, both definitions of carrier density and carrier multiplication are equivalent for symmetry reasons. Treating the full set of graphene Bloch equations, we have microscopic access to the temporal evolution of the carrier density including the contributions of carrierlight, carrier-carrier, and carrier-phonon interactions. With this, appearance of $\mathrm{CM}$ and hCM can be investigated. Both processes sensitively depend on the initial doping as well as on the excitation strength. A multiplication of charge carriers only takes place, if there is an asymmetry between the impact excitation (IE) and the inverse process of Auger recombination (AR) - in favor of the first. In addition, IE has to be more efficient than the competing carrier-phonon scattering to avoid energy dissipation to the lattice [130.

Carrier multiplication: Figure 6.6(a) illustrates the temporal evolution of the $\mathrm{CM}$ as a function of initial doping at a fixed absorbed pump fluence of $0.3 \mu \mathrm{Jcm}^{-2}$. The fluence dependence is discussed in detail in the supplementary material. The surface plot reveals that doping reduces the efficiency of carrier multiplication: The lower the doping, the higher is the CM factor reaching values of up to approximately 1.7 for undoped graphene, cf. Fig. 6.6(b). CM can be observed for Fermi energies of up to $300 \mathrm{meV}$. The maximal CM lifetime is approximately $150 \mathrm{fs}$ for undoped graphene and becomes significantly shorter for increasing doping, as illustrated in cf. Fig. 6.6(c). The observed carrier multiplication in the low-doping case can be explained by the strongly efficient impact excitation prevailing over the Auger recombination, which is a result of the large gradient in carrier occupation around the Dirac point, cf. Fig. 6.1(a). For undoped graphene, the probability for IE can be written as $\rho^{v}\left(1-\rho^{c}\right) \approx 1$, whereas the probability for $\mathrm{AR}$ is given by $\rho^{c}\left(1-\rho^{v}\right) \approx 0$. In this case, IE is significantly favored by Pauli-blocking during the initial dynamics, which is reflected by the corresponding rates $\gamma_{I E}$ and $\gamma_{A R}$, cf. Fig. 6.7(a) displaying the direct comparison between the temporal evolution of IE and AR rates after an optical excitation. The time-dependent generation $\gamma_{I E}$ and recombination $\gamma_{A R}$ rates are defined by the integration of the carrier-carrier scattering equation over all processes that increase or decreases the carrier density. The IE rate is clearly higher for a time range of approximately $100 \mathrm{fs}$ determining the strength of the appearing CM. During the carrier relaxation both rates converge to the same stationary limit and end up in an equilibrium, where no more 
Chapter 6. Impact of doping on carrier relaxation dynamics in graphene

carriers are generated. The lifetime of the $\mathrm{CM}$ is determined by the duration of the imbalance between IE and AR rates in combination with the interplay with competing channels of carrier-phonon scattering transfering the energy from the electronic system to the lattice. With an increasing doping, Auger scattering becomes more and more Pauli-blocked resulting in overall lower rates, cf. Fig. 6.7(b). Furthermore, for doping levels higher than $300 \mathrm{meV}$, AR becomes the dominant relaxation channels and CM does not appear anymore.

Hot carrier multiplication: The surface plot in Fig. 6.8(a) illustrates hCM as a function of doping and time at a fixed absorbed pump fluence of $\varepsilon_{\text {abs }}=$ $0.3 \mu \mathrm{Jcm}^{-2}$. In contrast to the behavior of $\mathrm{CM}$, we observe a clear increase of hCM with doping. Hot carrier multiplication factors of up to approximately 2 are found (at the considered pump fluence) with a lifetime of about $200 \mathrm{fs}$ for highly doped graphene with $\varepsilon_{F}=300 \mathrm{meV}$, cf. Figs. 6.8(b) and (c). There is nearly a linear dependence between $\mathrm{hCM}$ and doping: The smaller doping, the smaller is hCM and the shorter is its lifetime. The probability for intraband IE processes is given by $\rho^{h, c}\left(1-\rho^{e, c}\right)$, which is initially large compared to the probability for intraband AR processes $\rho^{e, c}\left(1-\rho^{h, c}\right)$. With increasing doping, the intraband Auger processes are shifted into a region of higher density of states making them more efficient, as reflected by the much higher scattering rates displayed in Fig. 6.7(b). The initial strong imbalance between $\tilde{\gamma}_{A R}$ and $\tilde{\gamma}_{I E}$ gives rise to a pronounced hCM. Besides the discussed doping dependence, $\mathrm{CM}$ or hCM are strongly sensitive to the excitation regime. A detailed discussion is provided in the next Section 6.3.1, where also a semi-analytical approach is presented focusing on the purely Coulomb-induced $\mathrm{CM}$ and hCM.

Direct comparison to experimental data: After having presented the theoretical results on the doping dependence of the carrier multiplication, a direct comparison with recently performed time-resolved ARPES measurements on nand p-doped graphene samples is presented [128]. Considering that the microscopic theory does not contain any fitting parameters, an excellent agreement between theory and experiment is obtained, cf. the inset in Fig. 6.8(c). There is a clearly higher hCM for n-doped graphene reaching values of up to 2.2 in the theory and more than 3 in the experiment. In contrast, for p-doped graphene only a small hCM of 1.4 (1.2) is obtained in theory and experiment, respectively. This pronounced difference is actually not due to the type of doping $(n, p)$. It can be clearly explained by the differences in the applied fluence and the doping strength. Note that at exactly same conditions with respect to $\varepsilon_{F}$ and $\varepsilon_{\text {abs }}$, the same carrier multiplication for both $\mathrm{n}$ - and p-doped samples are obtained. However, the experiment has been performed for: (i) n-doped graphene with the Fermi level $\varepsilon_{F}=$ 
Chapter 6. Impact of doping on carrier relaxation dynamics in graphene
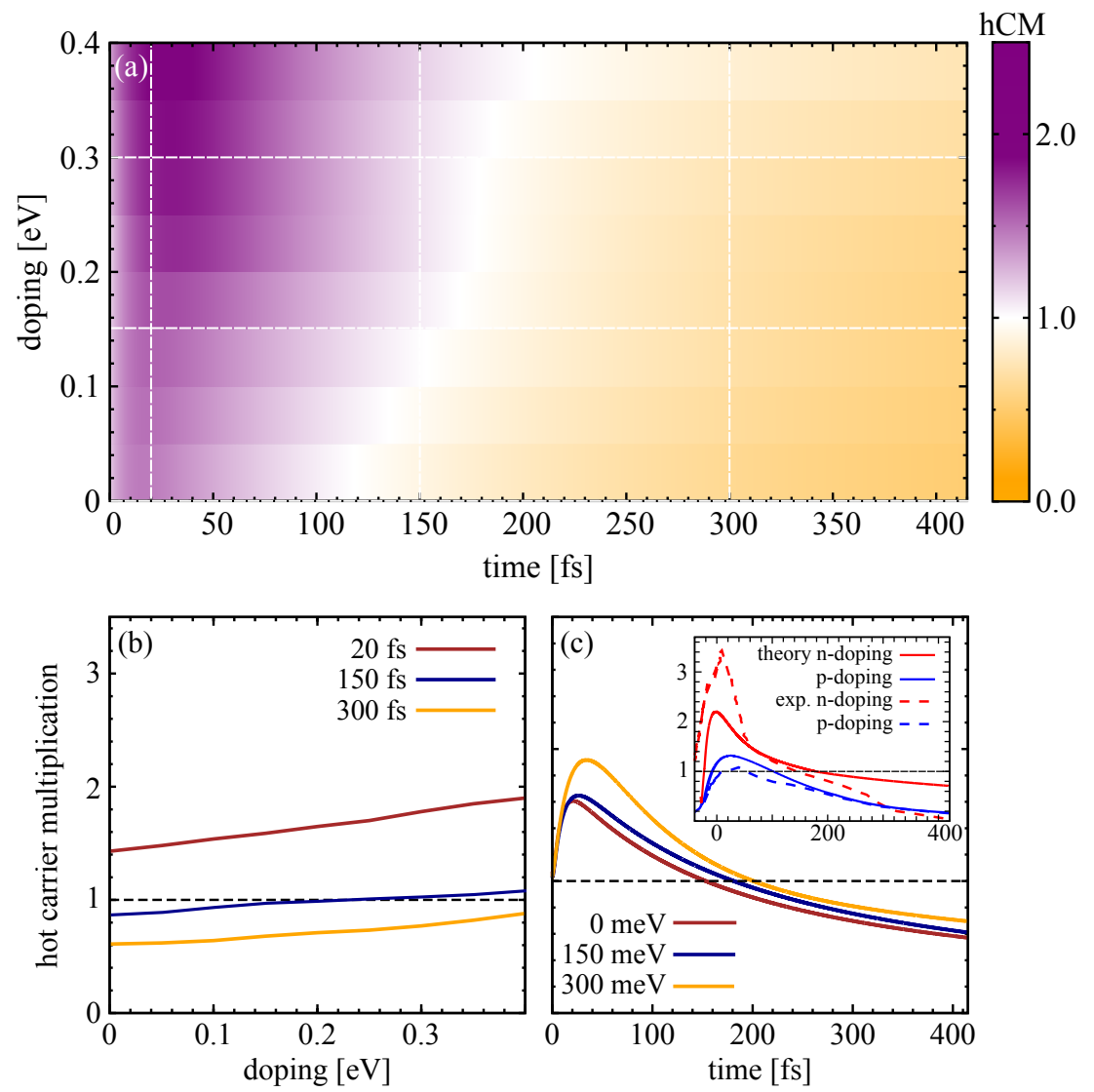

Figure 6.8.: (a) Temporal evolution of the doping-dependent hot carrier multiplication (hCM) for a fixed absorbed pump fluence of $\varepsilon_{\mathrm{abs}}=0.3 \mu \mathrm{Jcm}^{-2}$ and an excitation energy of $1.5 \mathrm{eV}$. (b) Doping-dependent CM for three fixed time delays and (c) the temporal evolution of $\mathrm{CM}$ for three fixed doping levels. The inset in (c) illustrates a direct comparison between theoretically predicted (solid lines) and experimentally measured (dashed lines) data for: (i) n-doped graphene $\left(\varepsilon_{F}=\right.$ $380 \mathrm{meV}$ ) with an absorbed pump fluence of $\varepsilon_{\mathrm{abs}}=0.5 \mu \mathrm{Jcm}^{-2}$ and (ii) p-doped graphene $\left(\varepsilon_{F}=240 \mathrm{meV}\right)$ with a fluence of $\varepsilon_{\mathrm{abs}}=1.5 \mu \mathrm{Jcm}^{-2}$. The experimental data is taken from Johannsen et al. [128]. Note that to be able to perform the comparison, the definition of the hCM has been adjusted taking into account only the contribution of the electrons. Figure is taken from [137.

$380 \mathrm{meV}$ and an absorbed pump fluence of $\varepsilon_{\mathrm{abs}}=0.5 \mu \mathrm{Jcm}^{-2}$ and (ii) p-doped graphene with $\varepsilon_{F}=240 \mathrm{meV}$ and $\varepsilon_{\text {abs }}=1.5 \mu \mathrm{Jcm}^{-2}$. As shown in Fig. 6.8(b), hot carrier multiplication increases almost linearly with the doping. Furthermore, it is strongly suppressed in the strong excitation regime, i.e. the larger the pump fluence, the less efficient is the hot carrier multiplication, as illustrated in Fig. 
Chapter 6. Impact of doping on carrier relaxation dynamics in graphene

6.11 in Section 6.3.1. As a result, the n-doped graphene sample shows a much more pronounced hCM, since its Fermi level $\varepsilon_{F}$ is significantly higher and since the experiment has been performed at a clearly smaller pump fluence compared to the p-doped graphene sample.

\subsubsection{Statictical approach for the purely Coulomb-induced carrier multiplication}

In order to find a favorable excitation and doping regime for carrier multiplication $(\mathrm{CM})$ and hot carrier multiplication $(\mathrm{hCM})$ the result of the purely Coulombinduced dynamics within statistical methods is presented. Carrier multiplication can be obtained analytically by using the carrier density $n$ and energy density $\mathcal{E}$ :

$$
n_{\mathrm{CM}}=\frac{\sigma_{s} \sigma_{v}}{L^{2}} \sum_{\lambda=e, h ; \mathbf{k}} \rho_{\mathbf{k}}^{\lambda}, \text { and } \mathcal{E}_{\mathrm{CM}}=\frac{\sigma_{s} \sigma_{v}}{L^{2}} \sum_{\lambda \mathbf{k}} \varepsilon_{\mathbf{k}}^{\lambda} \rho_{\mathbf{k}}^{\lambda}
$$

In equilibrium the occupation is given by a Fermi distribution and both carrier and energy density are functions of the temperature $T$ and the Fermi level $\varepsilon_{F}$. Therefore, it is possible to determine the Coulomb-induced final state of the system after an optical excitation. Assuming a Fermi distribution for the carrier occupation $\rho_{\mathbf{k}}^{\lambda}$, the carrier and energy density read in the case of $\varepsilon_{F} \neq 0$

$$
\begin{gathered}
n_{\mathrm{CM}}\left(T, \varepsilon_{F}\right)=-\frac{2 k_{B}^{2}}{\pi v_{F}^{2}} T^{2} \sum_{\sigma= \pm} \operatorname{Li}_{2}\left(-e^{\sigma \varepsilon_{F} / T k_{B}}\right), \\
\mathcal{E}_{\mathrm{hCM}}\left(T, \varepsilon_{F}\right)=-\frac{4 k_{B}^{3}}{\pi v_{F}^{2}} T^{3} \sum_{\sigma= \pm} \operatorname{Li}_{3}\left(-e^{\sigma \varepsilon_{F} / T k_{B}}\right),
\end{gathered}
$$

where $\operatorname{Li}_{n}($.$) denotes the polylogarithm. In the case of the hot carrier multiplication$ the carrier and energy density read

$$
\begin{aligned}
& n_{\mathrm{hCM}}\left(T, \varepsilon_{F}\right)=\frac{\sigma_{s} \sigma_{v}}{L^{2}}\left(\sum_{\mathbf{k}} \rho_{\mathbf{k}}^{h}+\sum_{\mathbf{k}}^{k<k_{F}} \rho_{\mathbf{k}}^{h, c}+\sum_{\mathbf{k}}^{k>k_{F}} \rho_{\mathbf{k}}^{e, c}\right), \\
& \mathcal{E}_{\mathrm{hCM}}\left(T, \varepsilon_{F}\right)=\frac{\sigma_{s} \sigma_{v}}{L^{2}}\left(\sum_{\mathbf{k}} \varepsilon_{\mathbf{k}}^{h} \rho_{\mathbf{k}}^{h}+\sum_{\mathbf{k}}^{k<k_{F}} \varepsilon_{\mathbf{k}}^{h, c} \rho_{\mathbf{k}}^{h, c}+\sum_{\mathbf{k}}^{k>k_{F}} \varepsilon_{\mathbf{k}}^{e, c} \rho_{\mathbf{k}}^{e, c}\right)
\end{aligned}
$$

with $\varepsilon_{\mathbf{k}}^{h, c}=\hbar v_{F}\left(k_{F}-k\right)$ for $k<k_{F}, \varepsilon_{\mathbf{k}}^{e, c}=\hbar v_{F}\left(k-k_{F}\right)$ for $k>k_{F}$, and in the bottom cone with $\varepsilon_{\mathbf{k}}^{h, v} \equiv \varepsilon_{\mathbf{k}}^{h}=\hbar v_{F}\left(k+k_{F}\right)$. A more detailed description of the carrier and energy density can be found in appendix $G$. 


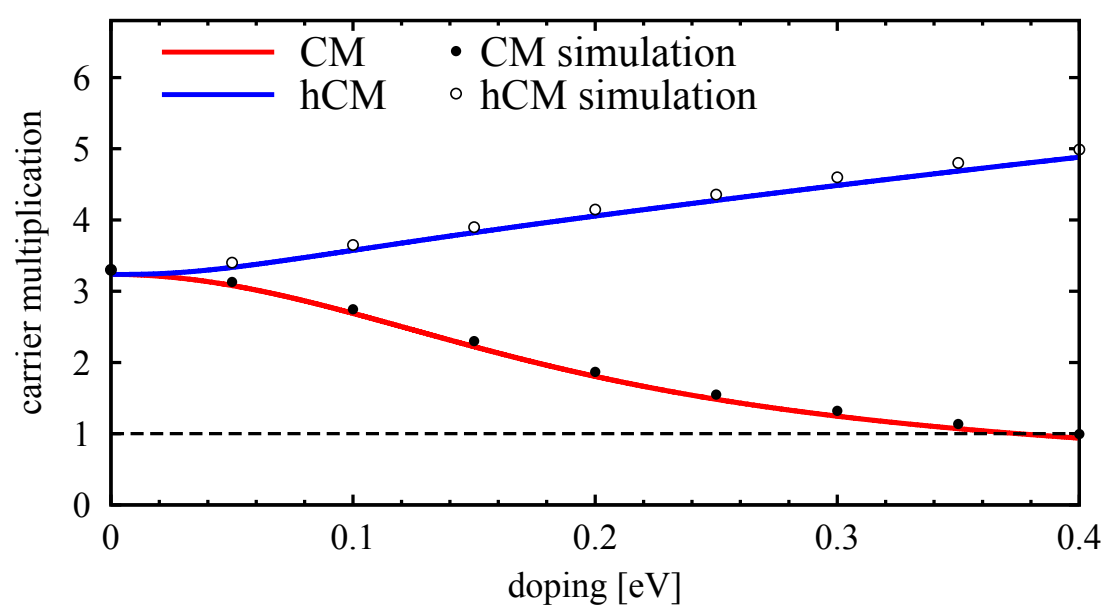

Figure 6.9.: Analytically obtained $\mathrm{CM}$ and hCM as a function of doping for a fixed pump fluence (solid line) compared to the numerical solution from the Bloch equations (dots). Figure is taken from 137 .

After an optical excitation the electronic system contains the carrier density $n=n\left(T_{0}, \varepsilon_{F}\right)+n_{\text {opt }}$ and the energy density $\mathcal{E}=\mathcal{E}\left(T_{0}, \varepsilon_{F}\right)+\mathcal{E}_{\text {opt }}$ with the initial temperature $T_{0}$ and $n_{\mathrm{opt}}$ and $\mathcal{E}_{\mathrm{opt}}$ are the optically excited carrier and energy density. Since Auger processes are considered, only the energy density will be conserved, whereas the carrier density is a variable quantity giving rise to a single Fermi distribution in the conduction and the valence band [130]. In constrast, Auger processes in a conventional semiconductor are negligible and the energy and the carrier density are both conserved and form new separate Fermi distributions in both bands. Starting with an initial thermal distribution at room temperature and a initial doping level $\varepsilon_{F}$, the energy density $\mathcal{E}\left(T_{\mathrm{exc}}, \varepsilon_{F}\right)=\mathcal{E}\left(T_{0}, \varepsilon_{F}\right)+\mathcal{E}_{\text {opt }}$ can be calculated to extract the excited temperature $T_{\text {exc }}$ and finally the final state of the system $f$ with $n_{f}=n\left(T_{\mathrm{exc}}, \varepsilon_{F}\right)$. The description of the final state made above is only valid without electron-phonon interaction and energy dissipation, which cannot be statiscally treated.

Fig. 6.9 shows an excellent agreement between the analytically [cf. Eqs. 6.5)[6.6] and numerically obtained doping-dependent carrier multiplication at a fixed pump fluence of $\varepsilon_{\mathrm{abs}}=0.1 \mu \mathrm{Jcm}^{-2}$. The higher the doping, the smaller is the CM and the larger is the hCM. For highly doped graphene samples with $\varepsilon_{F}>0.4 \mathrm{eV}$, $\mathrm{CM}$ becomes smaller than 1 . In contrast, hCM reaches values of almost 5 . The same qualitative dependence on doping is found by numerically evaluating the graphene Bloch equations (shown in the main part), which also include the carrierphonon coupling. This demonstrates that the presented semi-analytical approach 
Chapter 6. Impact of doping on carrier relaxation dynamics in graphene

already sufficiently covers the most important aspects of doping-dependent carrier multiplication. For quantitative insights and time-dependent $\mathrm{CM}$, the full microscopic approach is required. For a vanishing Fermi level, i.e. $\varepsilon_{F}=0$, both definitions of carrier and energy density are equivalent, see appendix G. In this case, carrier multiplication can be analytically expressed as a function of the absorbed pump fluence, photon energy $\hbar \omega_{L}$ and initial temperature $T_{0}$ [130:

$$
\operatorname{CM}\left(\mathcal{E}_{\text {opt }}, \omega_{L}, T_{0}\right)=\frac{a}{2} \frac{\hbar \omega_{L}}{\mathcal{E}_{\text {opt }}}\left[\left(T_{0}^{3}+b \mathcal{E}_{\text {opt }}\right)^{2 / 3}-T_{0}^{2}\right] .
$$

with the abbrevations $a=\pi v_{F}^{2} /\left(6 \zeta(3) k_{B}^{2}\right)$ and $b=\pi k_{B}^{2} /\left(3 v_{F}^{2}\right)$, where $\zeta($.$) denotes$ the Zeta function.

\section{Fluence dependence of carrier multiplication}

Now, based on the semi-analytical approach the impact of the excitation strength on the appearance of the CM and hCM is discussed. Figures 6.10(a) and 6.11(a) show surface plots illustrating the doping and the fluence dependence of CM and $\mathrm{hCM}$ at $300 \mathrm{~K}$ and at an excitation energy of $1.5 \mathrm{eV}$, respectively. The calculations demonstrate that both $\mathrm{CM}$ and hCM are strongly suppressed in the strong excitation regime. For pump fluences larger than approximately $5 \mu \mathrm{Jcm}^{-2}$ (hot) carrier multiplication does not occur any more. Figures 6.10(b) and 6.11(b) also illustrate the decrease (increase) of $\mathrm{CM}(\mathrm{hCM})$ with the doping. The larger the pump fluence, the more scattering partners are available giving rise to a faster carrier dynamics [30]. This leads to a thermalized distribution within the first tens of fs and the asymmetry of impact excitation and Auger recombination rates favors the latter. As a result, carrier multiplication clearly decreases at enhanced pump fluences, cf. Figs. 6.10(c) and 6.11(c).

Overall, the optimal conditions for $\mathrm{CM}$ and hCM are small pump fluences, low initial temperature, and a high excitation energy. Increasing the Fermi level leads to higher hCM factors, whereas CM decreases at the same time. 

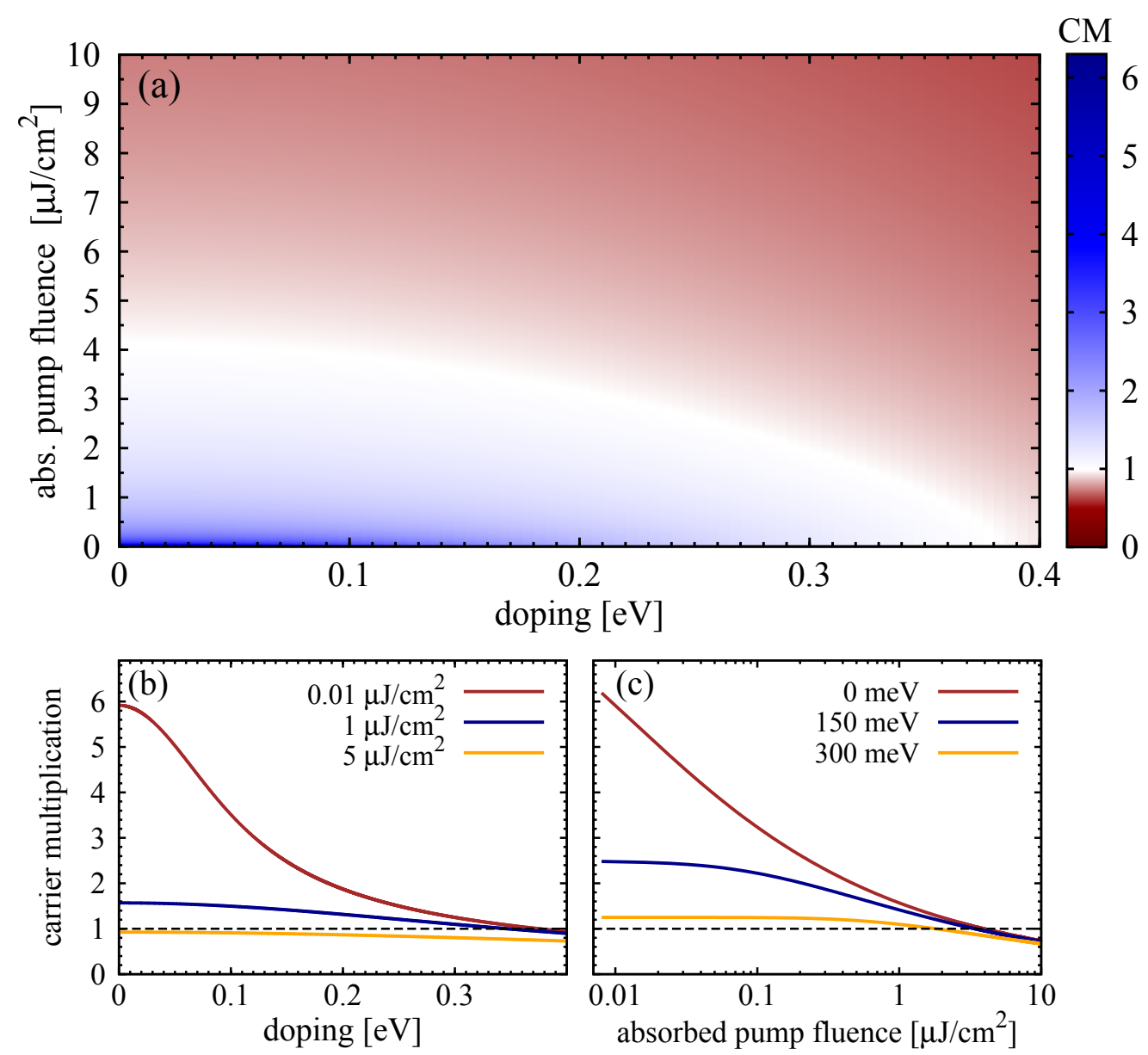

Figure 6.10.: (a) Doping-dependent carrier multiplication for different pump fluences at $300 \mathrm{~K}$ and an excitation energy of $1.5 \mathrm{eV}$. (b) Doping-dependent $\mathrm{CM}$ for three fixed pump fluences and (c) fluence-depenent CM for different doping levels. Figure is taken from 137. 

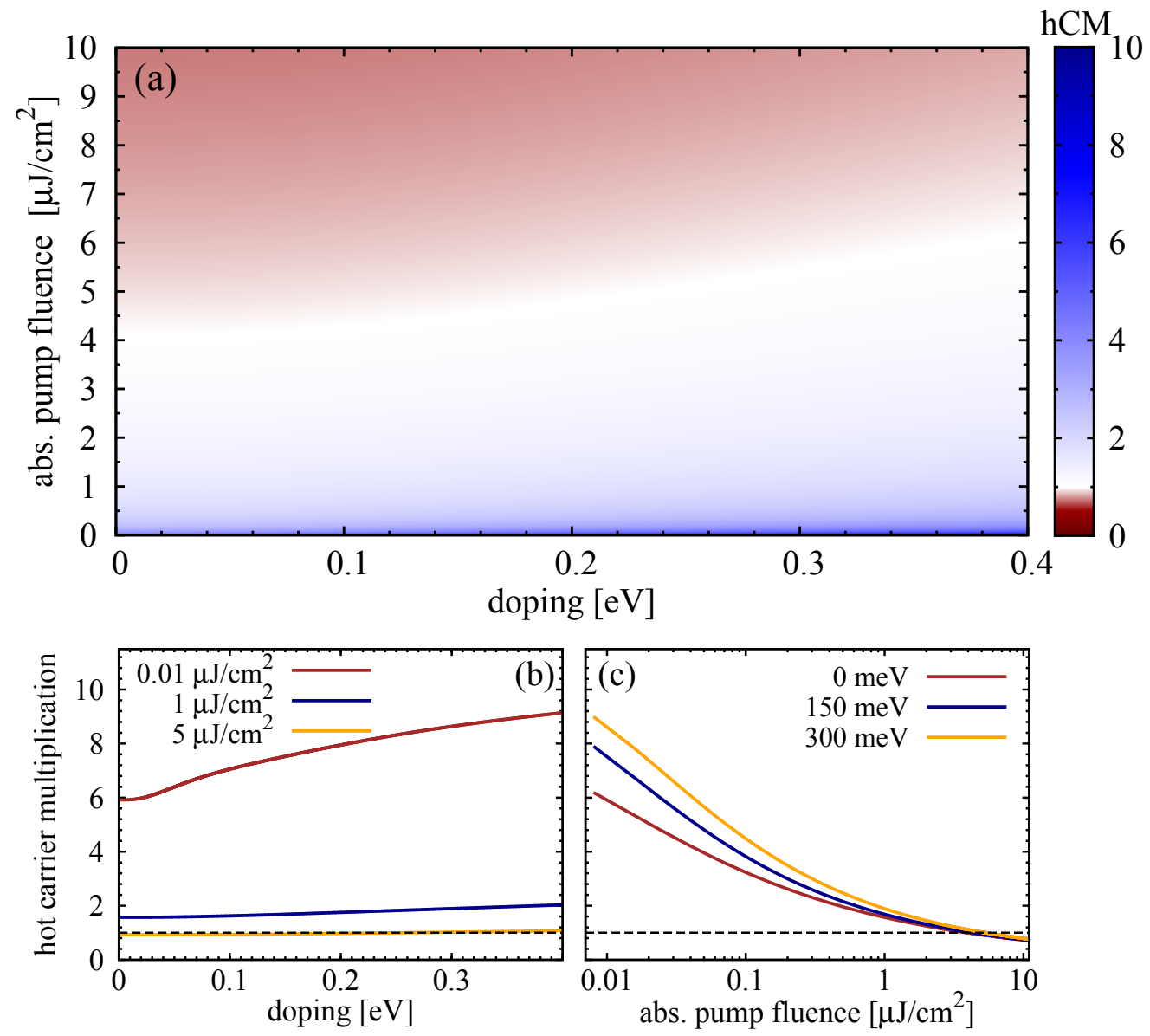

Figure 6.11.: (a) Doping-dependent hot carrier multiplication for different pump fluences at $300 \mathrm{~K}$ and an excitation energy of $1.5 \mathrm{eV}$. (b) Doping-dependent hCM for three fixed pump fluences and (c) fluence-depenent hCM for different doping levels. Figure is taken from 137 . 


\section{Conclusion}

In this thesis, a microscopic theoretical study of the ultrafast carrier dynamics in graphene is developed based on the density matrix formalism. The model accounts for a coherent description of optical excitation as well as Coulomb- and phonon-induced relaxation channels within the density matrix formalism. Based on a time-, momentum-, and angle-resolved access to carrier occupation, microscopic polarization and phonon population, this work has provided a thorough understanding of the nonequilibrium carrier dynamics in graphene. The graphene Bloch equations have been applied to explain linear absorption spectra and various features observed in optical pump-probe experiments.

To benchmark the theoretical approach, excitonic absorption spectra in monoand bilayer graphene have been performed. In agreement with recent experiments, the absorbance of graphene is characterized by a frequency-independent value in the near-infrared spectral region and a pronounced peak at $4.7 \mathrm{eV}$ resulting from interband transition. Bilayer graphene exhibits in the low energy spectrum a pronounced peak, which can be unambiguously ascribed to cross transitions $v_{1} \rightarrow c_{2}$ and $v_{2} \rightarrow c_{1}$ at the Dirac point. In the ultraviolet region, the spectrum is characterized by two pronounced energetically close absorption peaks resulting from interband transitions $v_{1} \rightarrow c_{1}$ and $v_{2} \rightarrow c_{2}$, respectively. For both mono- and bilayer graphene, the calculations reveal the importance of the Coulomb interaction resulting in the formation of saddle-point excitons with a binding energy of approximately $200 \mathrm{meV}$ for monolayer and $100 \mathrm{meV}$ for bilayer graphene. The analytic evaluation of the carrier-light coupling elements sheds light on the optical selection rules and the oscillator strength of the allowed optical transitions.

The theory proposes a microscopic mechanism explaining the occurrence of the transient negative differential transmission in graphene that has been observed in several recent pump-probe experiments. The calculations reveal that the interplay of interband and phonon-assisted intraband absorption processes provides a qualitative explanation of this effect, in particular phonon-assisted optical transitions give rise to an enhanced absorption in the excited carrier-phonon system. Phononinduced processes open an additional absorption channel assisted by simultaneous absorption or emission of phonons that are required to fulfil the conservation of momentum.

The calculate terahertz carrier dynamics depend critically on the Fermi level and range from a few picoseconds for high doping densities to hundreds of pi- 
coseconds for very low doping densities. In excellent agreement with optical pump and terahertz probe experiments, the $\mathrm{THz}$ carrier dynamics of doped and undoped graphene are qualitatively explained by the interplay of carrier-carrier and carrier-phonon interactions, without the need of disorder effects or any other extrinsic mechanisms and without the need for free fitting parameters. The theory accounts explicitly for the time-dependent response of the hot carriers to the $\mathrm{THz}$ probe field. Qualitatively, for highly doped graphene, in both experiment and theory a fast and substrate-temperature-independent $\mathrm{THz}$ carrier dynamics were observed. On the other hand, for graphene having very low doping density, a comparatively slower and strongly substrate-temperature-dependent $\mathrm{THz}$ carrier dynamics were obtained. A Drude model approximation to the full microscopic theory can provide only a semi-qualitative framework for interpreting the experimental data in doped and pristine graphene at low excitation fluences. However, the full microscopic theory is required to explain the data qualitatively for all excitation levels.

Introducing doping, the carrier dynamics in graphene exhibit interesting features. It could be shown, that the orientational relaxation dynamics in doped graphene is almost suppressed, if the excitation energy is near the Fermi level. In this case, the emission of phonons is suppressed due to the strict Markovian energy conservation, since the constant dispersion energies of the optical phonons are too large for efficient scattering processes. Furthermore, the impact of doping on the (hot) carrier multiplication was investigated. The appearance of Augerinduced carrier multiplication up to dopings of $300 \mathrm{meV}$ has been revealed. In the case of hot carrier multiplication, which occurs within one band, doping is even more advantageous, since it increases the phase space by providing a large number of available scattering partners. The comparison of the time-dependent hot carrier multiplication for $\mathrm{n}$ - and $\mathrm{p}$-doped with experimental ARPES measurements showed an excellent agreement. The results contribute to a better understanding of the ultrafast carrier dynamics in realistic graphene samples and provide valuable insights into the technologically relevant carrier multiplication in graphene.

However, there are still features in the experimental data that require an extension of the applied model. The neglected effects of Coulomb-induced intraband absorption processes or energy renormalization, which could explain a more pronounced fluence-dependence of the temporal zero-crossing in the experimental DT signal. Furthermore, the iteratively obtained THz dynamics is only valid, if both carrier-carrier and carrier-phonon scattering taken into account. To gain further insight in the $\mathrm{THz}$ regime, the numerical resolution of the momentum- and angle-dependent carrier dynamics needs to be enlarged. Moreover, the full nonMarkovian treatment of the carrier dynamics should be performed to investigate the role of quantum mechanical memory effects. 



\section{A. Optical matrix elements}

In this appendix part the optical matrix is expressed, which is given within the representation of second quantization by 41,51

$$
\mathbf{M}_{\mathbf{k k}^{\prime}}^{\lambda \lambda^{\prime}}=\left\langle\Psi_{\mathbf{k}}^{\lambda}(\mathbf{r})\left|\nabla_{\mathbf{r}}\right| \Psi_{\mathbf{k}^{\prime}}^{\lambda^{\prime}}(\mathbf{r})\right\rangle
$$

\section{A.1. Graphene}

Inserting tight-binding wave functions (Eq. 2.10), the matrix element reads

$$
\mathbf{M}_{\mathbf{k} \mathbf{k}^{\prime}}^{\lambda \lambda^{\prime}}=\frac{1}{N} \sum_{l, l^{\prime}}^{A, B} \sum_{\mathbf{R}_{l}, \mathbf{R}_{l^{\prime}}}\left(C_{l}^{\lambda *}(\mathbf{k}) C_{l^{\prime}}^{\lambda^{\prime}}\left(\mathbf{k}^{\prime}\right) e^{-i\left(\mathbf{k R}_{l}-\mathbf{k}^{\prime} \mathbf{R}_{l}^{\prime}\right)}\left\langle\phi\left(\mathbf{r}-\mathbf{R}_{l}\right)\left|\nabla_{\mathbf{k}}\right| \phi\left(\mathbf{r}-\mathbf{R}_{l^{\prime}}\right)\right\rangle+\text { c.c. }\right) .
$$

Applying the next-neighbour approximation and assuming that the $2 p_{z}$-orbital is symmetric in the $\mathrm{x}-\mathrm{y}$ plane and antisymmetric in the $z$ direction [48, 49], Eq. A.2 yields

$$
\begin{aligned}
\mathbf{M}_{\mathbf{k k}^{\prime}}^{\lambda \lambda^{\prime}}=\delta_{\mathbf{k k}^{\prime}} \sum_{i=1}^{3} & \left(C_{A}^{\lambda *}(\mathbf{k}) C_{B}^{\lambda^{\prime}}(\mathbf{k}) e^{i \mathbf{k} \mathbf{b}_{i}}\left\langle\phi\left(\mathbf{r}+\mathbf{b}_{i}\right)\left|\nabla_{\mathbf{k}}\right| \phi(\mathbf{r})\right\rangle\right. \\
& \left.+C_{B}^{\lambda *}(\mathbf{k}) C_{A}^{\lambda^{\prime}}(\mathbf{k}) e^{-i \mathbf{k} \mathbf{b}_{i}}\left\langle\phi(\mathbf{r})\left|\nabla_{\mathbf{k}}\right| \phi\left(\mathbf{r}+\mathbf{b}_{i}\right)\right\rangle\right) .
\end{aligned}
$$

The remaining integral can be determined by a rotation of the coordinate system resulting in $\left\langle\phi\left(\mathbf{r}+\mathbf{b}_{i}\right)\left|\nabla_{\mathbf{k}}\right| \phi(\mathbf{r})\right\rangle=M \frac{\mathbf{b}_{i}}{\left|\mathbf{b}_{i}\right|}|50|$. The integral $M$ can be determined numerically by expressing $2 p_{z}$ orbitals $\psi_{210}(\mathbf{r})=\sqrt{\frac{1}{4 \pi}}\left(\frac{Z_{\text {eff }}}{2 a_{B}}\right)^{\frac{3}{2}} \frac{Z_{\text {eff }}}{a_{B}} e^{\frac{Z_{\text {eff }}}{2 a_{B}}|\mathbf{r}|} z$, where $Z_{\text {eff }}$ is the effective charge number and $a_{B}$ the Bohr radius:

$$
\begin{aligned}
M & =\left\langle\psi\left(\begin{array}{c}
x+b \\
y \\
z
\end{array}\right)\left|\partial_{x}\right| \psi\left(\begin{array}{c}
x \\
y \\
z
\end{array}\right)\right\rangle \approx \int_{\infty}^{\infty} \mathrm{d} \mathbf{r} \psi_{210}\left(\begin{array}{c}
x+b \\
y \\
z
\end{array}\right) \partial_{x} \psi_{210}\left(\begin{array}{l}
x \\
y \\
z
\end{array}\right) \\
& \approx 3,00145 \mathrm{~nm}^{-1}
\end{aligned}
$$


Therewith the matrix element reads

$$
\mathbf{M}_{\mathbf{k k}^{\prime}}^{\lambda \lambda^{\prime}}=\delta_{\mathbf{k} \mathbf{k}^{\prime}} M \sum_{i=1}^{3}\left(C_{A}^{\lambda *}(\mathbf{k}) C_{B}^{\lambda^{\prime}}(\mathbf{k}) e^{i \mathbf{k} \mathbf{b}_{i}} \frac{\mathbf{b}_{i}}{\left|\mathbf{b}_{i}\right|}-C_{B}^{\lambda *}(\mathbf{k}) C_{A}^{\lambda^{\prime}}(\mathbf{k}) e^{-i \mathbf{k b}_{i}} \frac{\mathbf{b}_{i}}{\left|\mathbf{b}_{i}\right|}\right) .
$$

The tight-binding coefficients $C_{s}^{\lambda}(\mathbf{k})$ can be obtained by exploiting the normalization of the wave function $\left(1=\int \mathrm{d} \mathbf{r} \Psi_{\mathbf{k}}^{\lambda} \Psi_{\mathbf{k}}^{* \lambda}=\left|C_{A}^{\lambda}(\mathbf{k})\right|^{2}+\left|C_{B}^{\lambda}(\mathbf{k})\right|^{2}\right)$ :

$$
C_{A}^{\lambda}(\mathbf{k})=\sigma_{\lambda} \frac{1}{\sqrt{2}} \frac{e(\mathbf{k})}{|e(\mathbf{k})|}, \text { and } C_{B}^{\lambda}(\mathbf{k})=\sigma_{\lambda} \frac{1}{\sqrt{2}} .
$$

Finally, the interband matrix element is given by

$$
\mathbf{M}_{\mathbf{k k}^{\prime}}^{v c}=\delta_{\mathbf{k k}^{\prime}} \frac{M}{|e(\mathbf{k})|\left|b_{1}\right|} \operatorname{Re}\left[e^{*}(\mathbf{k}) \sum_{i=1}^{3} e^{i \mathbf{k b}_{i}} \mathbf{b}_{i}\right]
$$

and the intraband matrix element reads

$$
\mathbf{M}_{\mathbf{k k}^{\prime}}^{v v}=\delta_{\mathbf{k k}^{\prime}} \frac{i M}{|e(\mathbf{k})|\left|b_{1}\right|} \operatorname{Im}\left[e^{*}(\mathbf{k}) \sum_{i=1}^{3} e^{i \mathbf{k b}_{i}} \mathbf{b}_{i}\right]
$$

with the symmetry relations $\mathbf{M}_{\mathbf{k} \mathbf{k}^{\prime}}^{v c}=-\mathbf{M}_{\mathbf{k} \mathbf{k}^{\prime}}^{c v}$ and $\mathbf{M}_{\mathbf{k} \mathbf{k}^{\prime}}^{v v}=-\mathbf{M}_{\mathbf{k} \mathbf{k}^{\prime}}^{c c}$.

\section{A.1.1. Linearised matrix elements in graphene}

Within the linear regime of the band structure the optical matrix element can be further simplified. In this case, the function $e(\mathbf{k})$ can be expanded up to the first order around the Dirac point via

$$
e(\mathbf{k}) \approx-\frac{a_{0} \sqrt{3}}{2}\left(i \tilde{k}_{x}+\tilde{k}_{y}\right)
$$

Therewith, the simplified inter- and intraband matrix elements read

$$
\begin{aligned}
\mathbf{M}_{\mathbf{k}}^{v c} & =\frac{M}{|e(\mathbf{k})|\left|b_{1}\right|} \operatorname{Re}\left[e^{*}(\mathbf{k}) \sum_{i=1}^{3} e^{i \mathbf{k} \mathbf{b}_{i}} \mathbf{b}_{i}\right]=\frac{M}{|e(\mathbf{k})|\left|b_{1}\right|} \operatorname{Re}\left[e^{*}(\mathbf{k}) i \nabla_{\mathbf{k}} e(\mathbf{k})\right] \\
& =\frac{3}{2} M\left(\begin{array}{c}
\sin \varphi_{k} \\
\cos \varphi_{k}
\end{array}\right) \\
\mathbf{M}_{\mathbf{k}}^{v v} & =i \frac{3}{2} M\left(\begin{array}{c}
\cos \varphi_{k} \\
\sin \varphi_{k}
\end{array}\right) .
\end{aligned}
$$

The intraband matrix element is approximately a radial field, whereas the interband matrix element can be seen as a rotational field. 


\section{A.2. Bilayer graphene}

In analogy to graphene, the optical matrix element for bilayer graphene reads within the next-neighbour approximation

$$
\begin{aligned}
& \mathbf{M}_{\mathbf{k k}^{\prime}}^{\lambda \lambda^{\prime}}=\delta_{\mathbf{k}, \mathbf{k}^{\prime}} \sum_{i=1}^{3}\left(C_{A_{1}}^{\lambda *} C_{B_{1}}^{\lambda^{\prime}} e^{i \mathbf{k} \mathbf{b}_{i}}\left\langle\phi\left(\mathbf{r}+\mathbf{b}_{i}\right)\left|\nabla_{\mathbf{r}}\right| \phi(\mathbf{r})\right\rangle+C_{B_{1}}^{\lambda *} C_{A_{1}}^{\lambda^{\prime}} e^{-i \mathbf{k b}_{i}}\left\langle\phi(\mathbf{r})\left|\nabla_{\mathbf{r}}\right| \phi\left(\mathbf{r}+\mathbf{b}_{i}\right)\right\rangle\right. \\
& \left.+C_{A_{2}}^{\lambda *} C_{B_{2}}^{\lambda^{\prime}} e^{-i \mathbf{k} \mathbf{b}_{i}}\left\langle\phi(\mathbf{r})\left|\nabla_{\mathbf{r}}\right| \phi\left(\mathbf{r}+\mathbf{b}_{i}\right)\right\rangle+C_{B_{2}}^{\lambda *} C_{A_{2}}^{\lambda^{\prime}} e^{-i \mathbf{k} \mathbf{b}_{i}}\left\langle\phi\left(\mathbf{r}+\mathbf{b}_{i}\right)\left|\nabla_{\mathbf{r}}\right| \phi(\mathbf{r})\right\rangle\right) .
\end{aligned}
$$

Here, all terms of the form $\left\langle\phi\left(\mathbf{r}+\mathbf{R}_{I_{j}}\right)\left|\nabla_{\mathbf{r}}\right| \phi\left(\mathbf{r}+\mathbf{R}_{I_{k}^{\prime}}\right)\right\rangle$ for $I=I^{\prime}$ with $j=k$ and $j \neq k$ as well as $I \neq I^{\prime}$ with $j \neq k$ are neglected. The coefficients $C_{s}^{\lambda}(\mathbf{k})$ are obtained by exploiting the normalization of the wave function $\Psi_{\mathbf{k}}^{\lambda}(\mathbf{r})$ :

$$
\begin{array}{ll}
C_{A_{1}}^{\lambda}(\mathbf{k})=\frac{1}{\beta_{\mathbf{k}}^{\sigma_{i}}}\left(\frac{\gamma_{1}-\sigma_{i} \sqrt{4|e(\mathbf{k})|^{2} \gamma_{0}^{2}+\gamma_{1}^{2}}}{2 e(\mathbf{k}) \gamma_{0}}\right), \quad C_{B_{1}}^{\lambda}(\mathbf{k})=\sigma_{\lambda} \frac{1}{\beta_{\mathbf{k}}^{\sigma_{i}}}\left(\sigma_{i} \frac{e(\mathbf{k})^{*}}{e(\mathbf{k})}\right), \\
C_{A_{2}}^{\lambda}(\mathbf{k})=\sigma_{\lambda} \frac{1}{\beta_{\mathbf{k}}^{\sigma_{i}}}\left(\frac{\sigma_{i} \gamma_{1}-\sqrt{4|e(\mathbf{k})|^{2} \gamma_{0}^{2}+\gamma_{1}^{2}}}{2 e(\mathbf{k}) \gamma_{0}}\right), \quad C_{B_{2}}^{\lambda}(\mathbf{k})=\frac{1}{\beta_{\mathbf{k}}^{\sigma_{i}}}
\end{array}
$$

with the normalization value $\beta_{\mathbf{k}}^{\sigma_{i}}=\left(4-\frac{4 \gamma_{1}}{\left(\gamma_{1}+\sigma_{i} \sqrt{4|e(\mathbf{k})|^{2} \gamma_{0}^{2}+\gamma_{1}^{2}}\right)}\right)^{\frac{1}{2}}$. Here, the notations $\sigma_{\lambda}=+1(-1)$ for $\lambda=v_{i}\left(\lambda=c_{i}\right)$ and $\sigma_{i}=+1(-1)$ for $i=1(i=2)$ are used. Including the specific coefficients $C_{s}^{\lambda}(\mathbf{k})$ into Eq. A.12 and applying the relation $\left\langle\phi\left(\mathbf{r}+\mathbf{b}_{i}\right)\left|\nabla_{\mathbf{r}}\right| \phi(\mathbf{r})\right\rangle=M \frac{\mathbf{b}_{i}}{\left|\mathbf{b}_{i}\right|}$, the bilayer graphene optical matrix elements read

$$
\begin{aligned}
& \mathbf{M}_{\mathbf{k}}^{v_{i} c_{i}}=-\mathbf{M}_{\mathbf{k}}^{c_{i} v_{i}}=\frac{2 M \gamma_{0}}{\sqrt{4|e(\mathbf{k})|^{2} \gamma_{0}^{2}+\gamma_{1}^{2}}} \operatorname{Re}[\eta(\mathbf{k})], \\
& \mathbf{M}_{\mathbf{k}}^{v_{i} v_{i}}=-\mathbf{M}_{\mathbf{k}}^{c_{i} c_{i}}=-\frac{2 i M \gamma_{0}}{\sqrt{4|e(\mathbf{k})|^{2} \gamma_{0}^{2}+\gamma_{1}^{2}}} \operatorname{Im}[\eta(\mathbf{k})] \\
& \mathbf{M}_{\mathbf{k}}^{\lambda_{1} \lambda_{2}}=-\mathbf{M}_{\mathbf{k}}^{\lambda_{2} \lambda_{1}}=\frac{M \gamma_{1}}{|e(\mathbf{k})|^{2} \gamma_{0}}\left(1+\frac{\gamma_{1}^{2}}{|e(\mathbf{k})|^{2} \gamma_{0}^{2}}\right)^{-\frac{1}{2}} \operatorname{Re}[\eta(\mathbf{k})], \\
& \mathbf{M}_{\mathbf{k}}^{v_{1} c_{2}}=-\mathbf{M}_{\mathbf{k}}^{v_{2} c_{1}}=\frac{i M \gamma_{1}}{|e(\mathbf{k})|^{2} \gamma_{0}}\left(1+\frac{\gamma_{1}^{2}}{|e(\mathbf{k})|^{2} \gamma_{0}^{2}}\right)^{-\frac{1}{2}} \operatorname{Im}[\eta(\mathbf{k})],
\end{aligned}
$$

where $\eta(\mathbf{k})=e^{*}(\mathbf{k}) \sum_{i=1}^{3} e^{i \mathbf{k b}_{i}} \frac{\mathbf{b}_{i}}{\left|\mathbf{b}_{i}\right|}$. The matrix elements fulfil the symmetries $\mathbf{M}_{\mathbf{k}}^{v_{1} c_{2}}=\mathbf{M}_{\mathbf{k}}^{c_{2} v_{1}}=-\mathbf{M}_{\mathbf{k}}^{c_{1} v_{2}}$. 


\section{B. Coulomb matrix element}

The Coulomb matrix element is given by

$$
V_{l_{3} l_{4}}^{l_{1} l_{2}}=\int \mathrm{d} \mathbf{r} \int \mathrm{d} \mathbf{r}^{\prime} \Psi_{l_{1}}^{*}(\mathbf{r}) \Psi_{l_{2}}^{*}\left(\mathbf{r}^{\prime}\right) V_{\text {Coul }}\left(\mathbf{r}-\mathbf{r}^{\prime}\right) \Psi_{l_{4}}(\mathbf{r}) \Psi_{l_{3}}\left(\mathbf{r}^{\prime}\right),
$$

where $V_{\text {Coul }}\left(\mathbf{r}-\mathbf{r}^{\prime}\right)=\frac{e_{0}^{2}}{4 \pi \varepsilon_{0}} \frac{1}{\left|\mathbf{r}-\mathbf{r}^{\prime}\right|}$ is the screened Coulomb potential depending on a three-dimensional vector. Since all electron wave vectors lie in the graphene plane due to the small extent in the $z$ direction, the Coulomb potential is expanded in a two-dimensional Fourier series

$$
\begin{aligned}
V(\mathbf{r}) & =\sum_{\mathbf{q}} V_{\mathbf{q}} e^{i \mathbf{q r}} \\
\text { with } V_{\mathbf{q}} & =\frac{1}{L^{2}} \int_{A} \mathrm{~d} r^{\prime 2} V(\mathbf{r}) e^{-i \mathbf{q r}}=\frac{e_{0}^{2}}{4 \pi \varepsilon_{0} L^{2}} \int_{0}^{\infty} \mathrm{d} r \int_{0}^{2 \pi} \mathrm{d} \phi e^{-i q r \cos \phi} \\
& =\frac{e_{0}^{2}}{2 \varepsilon_{0} L^{2}} \frac{1}{q} .
\end{aligned}
$$

Therewith the potential yields

$$
V(\mathbf{r})=\frac{e_{0}^{2}}{2 \varepsilon_{0} \varepsilon_{r} L^{2}} \sum_{\mathbf{q}} \frac{1}{q} e^{-i \mathbf{q r}}
$$

with $q_{z}=0$. Now, Equation B.1 can be written as

$$
V_{l_{3} l_{4}}^{l_{1} l_{2}}=\sum_{\mathbf{q}} V_{\mathbf{q}} \Gamma_{l_{1} l_{3}} \Gamma_{l_{4} l_{2}}^{*}
$$

with $\Gamma_{i j}=\int_{V} \mathrm{~d} \mathbf{r} \Psi_{\mathbf{k}}^{i *}(\mathbf{r}) e^{i \mathbf{q r}} \Psi_{\mathbf{k}}^{j *}(\mathbf{r})$. Inserting the wave functions $\Psi_{\mathbf{k}}(\mathbf{r})$ from Eq. 2.10 into $\Gamma_{i j}$, the resulting integral yields

$$
\Gamma_{i j}=\left[C_{A}^{i *} C_{A}^{j}+C_{B}^{i *} C_{B}^{j}\right] I_{A}^{i j}(\mathbf{q}) \text { with } I_{A}^{i j}(\mathbf{q})=\int_{V} \mathrm{~d} \mathbf{r} \Phi_{A}^{i *}(\mathbf{k}, \mathbf{r}) e^{i \mathbf{q r}} \Phi_{A}^{j}(\mathbf{k}, \mathbf{r}) .
$$

Here, the overlap between different unit cells is neglected. Furthermore, the two remaining integrals yield the same value and can be expressed within the tightbinding ansatz:

$$
I_{A}^{i j}(\mathbf{q})=\int_{V} \mathrm{~d} \mathbf{r} \Phi_{A}^{i *}(\mathbf{k}, \mathbf{r}) e^{-i \mathbf{q r}} \Phi_{A}^{j}(\mathbf{k}, \mathbf{r})=\delta_{\mathbf{q}, \mathbf{k}_{i}-\mathbf{k}_{j}}\left[\left(\frac{q a_{B}}{Z_{\mathrm{eff}}}\right)^{2}+1\right]^{-3}
$$


with the Bohr radius $a_{B}$. The Kronecker delta is responsible for the conversation of the momentum. Finally, the Coulomb matrix element of graphene is given by

$$
V_{l_{3} l_{4}}^{l_{1} l_{2}}=V_{\mathbf{q}}\left[\left(\frac{q a_{B}}{Z_{e f f}}\right)^{2}+1\right]^{-6} \tilde{\Gamma}_{l_{1}, l_{3}} \tilde{\Gamma}_{l_{4}, l_{2}}^{*} \delta_{\mathbf{q}, \mathbf{k}_{4}-\mathbf{k}_{2}}
$$

with $\tilde{\Gamma}_{i j}=\left[C_{A}^{i *}(\mathbf{k}) C_{A}^{j}(\mathbf{k})+C_{B}^{i *}(\mathbf{k}) C_{B}^{j}(\mathbf{k})\right]$.

Bilayer graphene The integral $\Gamma_{i j}^{B G}$ reads in the case of bilayer graphene

$$
\begin{gathered}
\Gamma_{i j}^{B G}=\left[C_{A_{1}}^{i *} C_{A_{1}}^{j}+C_{B_{1}}^{i *} C_{B_{1}}^{j}+C_{A_{2}}^{i *} C_{A_{2}}^{j}+C_{B_{2}}^{i *} C_{B_{2}}^{j}\right] I_{A_{1}}^{i j}(\mathbf{q}) \\
\text { with } I_{A_{1}}^{i j}(\mathbf{q})=\int_{V} \mathrm{~d} \mathbf{r} \Phi_{A_{1}}^{i *}(\mathbf{k}, \mathbf{r}) e^{-i \mathbf{q r}} \Phi_{A_{1}}^{j}(\mathbf{k}, \mathbf{r})=\delta_{\mathbf{q}, \mathbf{k}_{i}-\mathbf{k}_{j}}\left[\left(\frac{q a_{B}}{Z_{\mathrm{eff}}}\right)^{2}+1\right]^{-3} .
\end{gathered}
$$

Inserting the integral $\Gamma_{i j}^{B G}$ into Eq. B.4 the bilayer graphene Coulomb matrix element is given by

$$
V_{l_{3} l_{4}}^{l_{1} l_{2}}=V_{\mathbf{q}}\left[\left(\frac{q a_{B}}{Z_{\text {eff }}}\right)^{2}+1\right]^{-6} \tilde{\Gamma}_{l_{1}, l_{3}}^{B G} \tilde{\Gamma}_{l_{4}, l_{2}}^{* B G}(\mathbf{q}) \delta_{\mathbf{q}, \mathbf{k}_{4}-\mathbf{k}_{2}}
$$

with $\tilde{\Gamma}_{i j}^{B G}=\sum_{\substack{s=A_{1}, A_{2} \\ B_{1}, B_{2}}} C_{s}^{i *}(\mathbf{k}) C_{s}^{j}(\mathbf{k})$. 


\section{Derivation of the Coulomb- and phonon-induced dynamics}

To access the carrier dynamics, the extension of the Bloch equations beyond the mean-field approximation is required. In this appendix, the scattering contribution of the microscopic quantities [see Eqs. 2.53 2.55] are derived within the secondorder Born-Markov approximation.

\section{C.1. Coulomb interaction}

Considering only the Coulomb interaction, the equation of motion for the quantity $\sigma_{12}=\left\langle a_{1}^{\dagger} a_{2}\right\rangle$ with the index $1=\left(\mathbf{k}_{1}, \lambda_{1}\right)$ is given by

$$
\left.i \hbar \frac{d}{d t} \sigma_{12}\right|_{H_{c, c}}=\sum_{a b c}\left[V_{b c}^{2 a} \Lambda_{c b}^{1 a}-V_{1 c}^{a b} \Lambda_{c 2}^{a b}\right]
$$

with the abbreviation $\Lambda_{34}^{12}=\left\langle a_{1}^{\dagger} a_{2}^{\dagger} a_{3} a_{4}\right\rangle$. These equation describes the meanfield Coulomb interaction of the transition $\sigma_{12}$ between the states 1 and 2, where the dynamics of the density matrix element couples to four-operators expectation values. Applying the correlation expansion for these expectation values, they can be divided into a Hartree-Fock contribution (HF) and a second-order correlation contribution (scat) with

$$
\begin{aligned}
\left.i \hbar \frac{d}{d t} \sigma_{12}\right|_{\mathrm{HF}} & =\sum_{a b c}\left[V_{b c}^{2 a}\left[\sigma_{1 b} \sigma_{a c}-\sigma_{1 c} \sigma_{a b}\right]-V_{1 c}^{a b}\left[\sigma_{a 2} \sigma_{b c}-\sigma_{a c} \sigma_{b 2}\right]\right] \\
\left.i \hbar \frac{d}{d t} \sigma_{12}\right|_{\mathrm{scat}} & =\sum_{a b c}\left[V_{b c}^{2 a} \Lambda_{c b}^{1 a^{c}}-V_{1 c}^{a b} \Lambda_{c 2}^{a b c}\right] .
\end{aligned}
$$

Here, $\Lambda_{34}^{12^{c}}$ denotes the correlated expectation value.

Carrier-Carrier scattering rates In this part the treatment of the Coulomb interaction goes beyond the Hartree-Fock level by deriving a Coulomb-induced Boltzmann-like equation within the second-order Born-Markov approximation. 
The scattering between two electrons is described by C.4

$$
\Lambda_{34}^{12^{c}}=\Lambda_{34}^{12}+\sigma_{13} \sigma_{24}-\sigma_{14} \sigma_{23}
$$

In order to determine their dynamics, we apply the Heisenberg equation of motion to equation C.4 for the Hamilton operators. The free particle Hamiltonian directly yields

$$
\left.\frac{d}{d t} \Lambda_{34}^{12^{c}}\right|_{H_{0}}=\frac{i}{\hbar} \varepsilon_{34}^{12} \Lambda_{34}^{12^{c}}
$$

with $\varepsilon_{34}^{12}=\varepsilon_{1}+\varepsilon_{2}-\varepsilon_{3}-\varepsilon_{4}$.

The Coulomb-induced dynamics of $\Lambda_{34}^{12}$ in Eq. C.4 is split into two terms, where the temporal evolution of $\Lambda_{34}^{12}$ is given by

$$
\begin{aligned}
\left.i \hbar \frac{d}{d t} \Lambda_{34}^{12}\right|_{H_{c, c}} & =\sum_{a b c} V_{1 c}^{a b}\left[\left\langle a_{a}^{\dagger} a_{b}^{\dagger} a_{2}^{\dagger} a_{c} a_{3} a_{4}\right\rangle-\Lambda_{34}^{a b} \delta_{2 c}\right]-\sum_{a b c} V_{2 c}^{a b}\left\langle a_{a}^{\dagger} a_{b}^{\dagger} a_{1}^{\dagger} a_{c} a_{3} a_{4}\right\rangle \\
& +\sum_{a b c} V_{b c}^{3 a}\left\langle a_{1}^{\dagger} a_{2}^{\dagger} a_{a}^{\dagger} a_{4} a_{c} a_{b}\right\rangle-\sum_{a b c} V_{b c}^{4 a}\left[\left\langle a_{1}^{\dagger} a_{2}^{\dagger} a_{a}^{\dagger} a_{3} a_{c} a_{b}\right\rangle-\Lambda_{c b}^{12} \delta_{3 a}\right]
\end{aligned}
$$

with the three-particle quantity $\left\langle a_{1}^{\dagger} a_{2}^{\dagger} a_{3}^{\dagger} a_{4} a_{5} a_{6}\right\rangle$. The factorization of the threeparticle quantity reads within the correlation expansion

$$
\begin{aligned}
\left\langle a_{1}^{\dagger} a_{2}^{\dagger} a_{3}^{\dagger} a_{4} a_{5} a_{6}\right\rangle \approx & \sigma_{14}\left(\sigma_{26} \sigma_{35}-\sigma_{25} \sigma_{36}\right)+\sigma_{15}\left(\sigma_{24} \sigma_{36}-\sigma_{26} \sigma_{34}\right)+\sigma_{16}\left(\sigma_{25} \sigma_{34}-\sigma_{24} \sigma_{35}\right) \\
& +\Lambda_{45}^{12^{c}} \sigma_{36}-\Lambda_{46}^{12^{c}} \sigma_{35}+\Lambda_{56}^{12^{c}} \sigma_{34}-\Lambda_{45}^{13^{c}} \sigma_{26}+\Lambda_{46}^{13^{c}} \sigma_{25} \\
& -\Lambda_{56}^{13^{c}} \sigma_{24}+\Lambda_{45}^{23^{c}} \sigma_{16}-\Lambda_{46}^{23^{c}} \sigma_{15}+\Lambda_{56}^{23^{c}} \sigma_{14} .
\end{aligned}
$$

The equation of motion of the second term of Eq. C.4 is given by

$$
\begin{aligned}
\left.i \hbar \frac{d}{d t}\left[\sigma_{13} \sigma_{24}-\sigma_{14} \sigma_{23}\right]\right|_{H_{c, c}}= & \sum_{a b c}\left\{V_{1 c}^{a b}\left[\Lambda_{c 4}^{a b} \sigma_{23}-\Lambda_{c 3}^{a b} \sigma_{24}\right]+V_{2 c}^{a b}\left[\Lambda_{c 4}^{a b} \sigma_{23}-\Lambda_{c 3}^{a b} \sigma_{24}\right]\right. \\
& \left.+V_{b c}^{3 a}\left[\Lambda_{c b}^{1 a} \sigma_{24}-\Lambda_{c b}^{2 a} \sigma_{14}\right]+V_{b c}^{4 a}\left[\Lambda_{c b}^{2 a} \sigma_{13}-\Lambda_{c b}^{1 a} \sigma_{23}\right]\right\} .
\end{aligned}
$$


Inserting Eqs. C.6 and C.8 into the correlated term C.4 the dynamics of $\Lambda_{34}^{12^{c}}$ yields

$$
\begin{aligned}
\left.i \hbar \frac{d}{d t} \Lambda_{34}^{12 c}\right|_{H_{c, c}} & =\sum_{a b c d}\left(V_{d c}^{a b}-V_{d c}^{b a}\right)\left[\sigma_{1 d} \sigma_{2 c} \sigma_{b 3}^{\dagger} \sigma_{a 4}^{\dagger}-\sigma_{1 d}^{\dagger} \sigma_{2 c}^{\dagger} \sigma_{b 3} \sigma_{a 4}\right] \\
& -\sum_{a}\left[\epsilon_{1 a}^{c o r} \Lambda_{34}^{a 2^{c}}+\epsilon_{2 a}^{c o r} \Lambda_{a 4}^{12 c}-\epsilon_{3 a}^{c o r^{*}} \Lambda_{a 4}^{12^{c}}-\epsilon_{4 a}^{c o r^{*}} \Lambda_{3 a}^{12^{c}}\right] \\
& +\sum_{a b c d} V_{d c}^{a b}\left[\Lambda_{34}^{a b^{c}}\left(\sigma_{1 d} \sigma_{2 c}-\sigma_{1 d}^{\dagger} \sigma_{2 c}^{\dagger}\right)+\Lambda_{c d}^{12^{c}}\left(\sigma_{b 3}^{\dagger} \sigma_{a 4}^{\dagger}-\sigma_{b 3} \sigma_{a 4}\right)\right] \\
& +2 \sum_{a b c d} V_{d c}^{a b}\left[\Lambda_{c 4}^{2 a}\left(\sigma_{1 d} \sigma_{3 b}^{\dagger}-\sigma_{b 3} \sigma_{d 1}^{\dagger}\right)+\Lambda_{c 3}^{2 a^{c}}\left(\sigma_{b 4} \sigma_{d 1}^{\dagger}-\sigma_{d 1} \sigma_{4 b}^{\dagger}\right)\right. \\
& \left.+\quad \Lambda_{d 4}^{a 1^{c}}\left(\sigma_{b 3} \sigma_{c 2}^{\dagger}-\sigma_{2 c} \sigma_{3 b}^{\dagger}\right)+\Lambda_{d 3}^{a 1^{c}}\left(\sigma_{2 c} \sigma_{4 b}^{\dagger}-\sigma_{b 4} \sigma_{c 2}^{\dagger}\right)\right]
\end{aligned}
$$

with $\epsilon_{1 a}^{\mathrm{cor}}=2 \sum_{b c} V_{1 c}^{a b} \sigma_{b c}$ and $\epsilon_{3 a}^{\mathrm{cor} *}=2 \sum_{b c} V_{c a}^{3 b} \sigma_{b c}$. To obtain the Boltzmann-like scattering equation only the terms from the first row in Eq. C.9 are relevant. The second line leads to a many-particle screening of the Coulomb-potential, see appendix D. Now, the dynamic of equation C.4 can be written as

$$
\frac{d}{d t} \Lambda_{34}^{12^{c}}=\left(\frac{i}{\hbar} \Delta \varepsilon_{34}^{12}-\gamma\right) \Lambda_{34}^{12^{c}}+\frac{i}{\hbar} Q_{34}^{12}(t)
$$

with a decay constant $\gamma$ describing the higher-order correlation beyond secondorder Born-Markov approximation and the scattering kernel

$$
Q_{34}^{12}(t)=\sum_{a b c d}\left(V_{d c}^{a b}-V_{d c}^{b a}\right)\left[\sigma_{1 d}^{\dagger} \sigma_{2 c}^{\dagger} \sigma_{b 3} \sigma_{a 4}-\sigma_{1 d} \sigma_{2 c} \sigma_{b 3}^{\dagger} \sigma_{a 4}^{\dagger}\right]
$$

Applying the Markov approximation the solution of the two-particle correlation is given by

$$
\Lambda_{34}^{12}=-i \pi Q_{34}^{12}(t) \delta\left(\Delta \varepsilon_{C D}^{A B}\right) .
$$

Inserting the solution of $\Lambda_{34}^{12}$ in Eq. C.3 results in

$$
\begin{aligned}
\left.\frac{d}{d t} \sigma_{12}\right|_{H_{c, c}} & =\frac{\pi}{\hbar} \sum_{a b c} V_{b c}^{2 a}\left[\sum_{A B C D} \tilde{V}_{D C}^{A B}\left[\sigma_{1 D}^{\dagger} \sigma_{a C}^{\dagger} \sigma_{B c} \sigma_{A b}-\sigma_{a D} \sigma_{b C} \sigma_{B c}^{\dagger} \sigma_{A b}^{\dagger}\right] \delta\left(\Delta \varepsilon_{C D}^{A B}\right)\right] \\
& -\frac{\pi}{\hbar} \sum_{a b c} V_{1 c}^{a b}\left[\sum_{A B C D} \tilde{V}_{D C}^{A B}\left[\sigma_{a D}^{\dagger} \sigma_{b C}^{\dagger} \sigma_{B c} \sigma_{A 2}-\sigma_{a D} \sigma_{b C} \sigma_{B c}^{\dagger} \sigma_{A 2}^{\dagger}\right] \delta\left(\Delta \varepsilon_{C D}^{A B}\right)\right]
\end{aligned}
$$

with $\tilde{V}_{D C}^{A B}=V_{D C}^{A B}-V_{D C}^{B A}$. 
Carrier Occupation The resulting microscopic Boltzmann-like kinetic equation for the carrier occupation reads

$$
\left.\dot{\rho}_{l}\right|_{H_{c, c}}=\Gamma_{l}^{\mathrm{in}, c c}\left[1-\rho_{l}\right]+\Gamma_{l}^{\mathrm{out}, c c}(t) \rho_{l},
$$

where the in- and out-scattering rates for the Coulomb-induced carrier dynamics are given by

$$
\begin{aligned}
\Gamma_{l}^{\mathrm{in}, c c} & =\frac{2 \pi}{\hbar} \sum_{a b c} V_{b c}^{l a} \tilde{V}_{b c}^{l a}\left[1-\rho_{a}\right] \rho_{b} \rho_{c} \delta\left(\varepsilon_{l}+\varepsilon_{a}-\varepsilon_{b}-\varepsilon_{c}\right), \\
\Gamma_{l}^{\mathrm{out}, c c} & =\frac{2 \pi}{\hbar} \sum_{a b c} V_{b c}^{l a} \tilde{V}_{b c}^{l a} \rho_{a}\left[1-\rho_{b}\right]\left[1-\rho_{c}\right] \delta\left(\varepsilon_{l}+\varepsilon_{a}-\varepsilon_{b}-\varepsilon_{c}\right)
\end{aligned}
$$

with $\tilde{V}_{b c}^{l a}=2 V_{b c}^{l a *}-V_{b c}^{a l *}$.

Polarization To obtain the Coulomb-induced dynamics of the microscopic polarization $p_{\mathbf{k}}(t)$ all terms in Eq. C.13 proportional to $p_{\mathbf{k}}$ (first order) are considered here. The resulting temporal evolution of $p_{\mathbf{k}}(t)$ yields

$$
\left.\frac{d}{d t} p_{\mathbf{k}}(t)\right|_{H_{c, c}}=-\gamma_{\mathbf{k}}^{c c} p_{\mathbf{k}}+\sum_{\mathbf{k}^{\prime}}\left[\mathcal{U}_{\mathbf{k k}^{\prime}}^{v c}(t) p_{\mathbf{k}^{\prime}}(t)+\mathcal{U}_{\mathbf{k k}^{\prime}}^{c v}(t) p_{\mathbf{k}^{\prime}}^{*}(t)\right]
$$

with the diagonal dephasing

$$
\gamma_{\mathbf{k}}^{c c}=\frac{1}{2} \sum_{\lambda}\left[\Gamma_{\lambda, \mathbf{k}}^{\mathrm{in}, c c}(t)+\Gamma_{\lambda, \mathbf{k}}^{\mathrm{out}, c c}(t)\right],
$$

and the off-diagonal dephasing

$$
\begin{aligned}
& \mathcal{U}_{\mathbf{k k}^{\prime}}^{\lambda_{1} \lambda_{2}}=\frac{\pi}{\hbar} \sum_{\mathbf{a b}} \sum_{\lambda}\left[V_{\mathbf{k}^{\prime} \lambda_{2} \mathbf{k}_{b} \lambda_{b}}^{\mathbf{k} c \mathbf{k}_{c} \lambda_{c}} \tilde{V}_{\mathbf{k} v \mathbf{k}_{c} \lambda_{c}}^{\mathbf{k}^{\prime} \lambda_{1} \mathbf{k}_{b} \lambda_{b}}\left(\rho_{\mathbf{k}}^{\lambda}\left[1-\rho_{\mathbf{b}}\right] \rho_{\mathbf{c}}+\left[1-\rho_{\mathbf{k}}^{\lambda}\right] \rho_{\mathbf{b}}\left[1-\rho_{\mathbf{c}}\right]\right) \delta_{c c}^{+}\right. \\
& \left.-V_{\mathbf{k}_{b} \lambda_{b} \mathbf{k}_{c} \lambda_{c}}^{\mathbf{k} c \mathbf{k}^{\prime} \lambda_{1}} \tilde{V}_{\mathbf{k} v \mathbf{k}^{\prime} \lambda_{2}}^{\mathbf{k}_{b} \lambda_{b} \mathbf{k}_{c} \lambda_{c}}\left(\left[1-\rho_{\mathbf{k}}^{\lambda}\right] \rho_{\mathbf{b}} \rho_{\mathbf{c}}+\rho_{\mathbf{k}}^{\lambda}\left[1-\rho_{\mathbf{b}}\right]\left[1-\rho_{\mathbf{c}}\right]\right) \delta_{c c}^{-}\right],
\end{aligned}
$$

where $\delta_{c c}^{ \pm}=\delta\left(\varepsilon_{\mathbf{k}}^{\lambda}+\sigma_{\lambda^{\prime}} \varepsilon_{\mathbf{k}^{\prime}}^{\lambda}-\varepsilon_{\mathbf{b}} \pm \varepsilon_{\mathbf{c}}\right)$.

\section{C.2. Electron-phonon coupling}

In the following, the electron-phonon scattering rates are derived. Note, that spatial homogeneity is applied via $\left(\sigma_{12}=\left\langle a_{1}^{\dagger} a_{2}\right\rangle \delta_{\mathbf{k}_{1}, \mathbf{k}_{2}}=\sigma_{\mathbf{k}}^{\lambda \lambda^{\prime}}\right)$. Applying the 
Heisenberg equation of motion the dynamics for the density matrix element $\sigma_{\mathbf{k}}^{\lambda \lambda^{\prime}}$ reads

$$
\left.\dot{\sigma}_{\mathbf{k}}^{\lambda \lambda^{\prime}}\right|_{H_{c, p}}=\frac{1}{i \hbar} \sum_{\lambda^{\prime}} \sum_{j \mathbf{q}}\left(g_{\mathbf{k}, \mathbf{q}}^{\lambda \lambda^{\prime} j} S_{\mathbf{k}, \mathbf{q}}^{\lambda \lambda^{\prime} j}-g_{\mathbf{k}+\mathbf{q}, \mathbf{q}}^{\lambda^{\prime} \lambda j} S_{\mathbf{k}+\mathbf{q}, \mathbf{q}}^{\lambda^{\prime} \lambda j}-g_{\mathbf{k}, \mathbf{q}}^{\lambda \lambda^{\prime} j *} T_{\mathbf{k}, \mathbf{q}}^{\lambda^{\prime} \lambda j}+g_{\mathbf{k}+\mathbf{q}, \mathbf{q}}^{\lambda^{\prime} \lambda j *} T_{\mathbf{k}+\mathbf{q}, \mathbf{q}}^{\lambda \lambda^{\prime} j}\right),
$$

where $S_{\mathbf{k}, \mathbf{q}}^{\lambda \lambda^{\prime} j}=\left\langle a_{\mathbf{k}}^{\lambda \dagger} a_{\mathbf{k}-\mathbf{q}}^{\lambda^{\prime}} b_{\mathbf{q}}^{j}\right\rangle$ and $T_{\mathbf{k}, \mathbf{q}}^{\lambda \lambda^{\prime} j}=\left\langle a_{\mathbf{k}-\mathbf{q}}^{\lambda \dagger} a_{\mathbf{k}}^{\lambda^{\prime}} b_{\mathbf{q}}^{j \dagger}\right\rangle$ are phonon-assisted electron densities and transitions [88|. The correlation expansion for the phonon-assisted quantities is considered via $S_{\mathbf{k}, \mathbf{q}}^{\lambda \lambda^{\prime} j}=\left\langle a_{\mathbf{k}}^{\lambda^{\dagger}} a_{\mathbf{k}-\mathbf{q}}^{\lambda^{\prime}}\right\rangle\left\langle b_{\mathbf{q}}^{j}\right\rangle+S_{\mathbf{k}, \mathbf{q}}^{\lambda \lambda^{\prime} j c}$. The coherent phonons $\left\langle b_{\mathbf{q}}^{j}\right\rangle$ will be neglected, since their influence on the relaxation dynamics in graphene is negligible. As a results, the phonon-assisted quantities and their correlation are equal. The temporal evolution of $S_{\mathbf{k}, \mathbf{q}}^{\lambda \lambda^{\prime} j}$ and $T_{\mathbf{k}, \mathbf{q}}^{\lambda \lambda^{\prime} j}$ stemming from the free particle and electron-phonon Hamiltonian yields

$$
\begin{aligned}
& \left.i \hbar \frac{d}{d t} S_{\mathbf{k}, \mathbf{q}}^{\lambda \lambda^{\prime} j}\right|_{H_{0}+H_{c, p}}=-\left(\varepsilon_{\mathbf{k}}^{\lambda}-\varepsilon_{\mathbf{k}-\mathbf{q}}^{\lambda^{\prime}}-\hbar \omega_{\mathbf{q}}^{j}\right) S_{\mathbf{k}, \mathbf{q}}^{\lambda \lambda^{\prime} j}-\sum_{\lambda_{3} \lambda_{4}} g_{\mathbf{k}, \mathbf{q}}^{\lambda_{3} \lambda_{4} j *} Q_{\mathbf{k}, \mathbf{q}}^{\lambda_{1} \lambda_{3} \lambda_{4} \lambda_{2} j} \\
& \left.i \hbar \frac{d}{d t} T_{\mathbf{k}, \mathbf{q}}^{\lambda \lambda^{\prime} j}\right|_{H_{0}+H_{c, p}}=\left(\varepsilon_{\mathbf{k}}^{\lambda}-\varepsilon_{\mathbf{k}-\mathbf{q}}^{\lambda^{\prime}}-\hbar \omega_{\mathbf{q}}^{j}\right) T_{\mathbf{k}, \mathbf{q}}^{\lambda \lambda^{\prime} j}+\sum_{\lambda_{3} \lambda_{4}} g_{\mathbf{k}, \mathbf{q}}^{\lambda_{3} \lambda_{4} j} Q_{\mathbf{k}, \mathbf{q}}^{\lambda_{3} \lambda_{2} \lambda_{1} \lambda_{4} j}
\end{aligned}
$$

with the scattering kernel $Q_{\mathbf{k}, \mathbf{q}}^{\lambda_{1} \lambda_{2} \lambda_{3} \lambda_{4} j}=\left(\delta_{\lambda_{1} \lambda_{2}}-\sigma_{\mathbf{k}}^{\lambda_{1} \lambda_{2}}\right) \sigma_{\mathbf{k}-\mathbf{q}}^{\lambda_{3} \lambda_{4}} n_{\mathbf{q}}^{j}-\sigma_{\mathbf{k}}^{\lambda_{1} \lambda_{2}}\left(\delta_{\lambda_{3} \lambda_{4}}-\right.$ $\left.\sigma_{\mathbf{k}-\mathbf{q}}^{\lambda_{3} \lambda_{4}}\right)\left(n_{\mathbf{q}}^{j}+1\right)$. In analogy to the Coulomb scattering rates, Eqs. C.21 and C.22 can be analytically solved within the Markov approximation yielding

$$
\begin{aligned}
& S_{\mathbf{k}, \mathbf{q}}^{\lambda \lambda^{\prime} j}=i \pi \sum_{\lambda_{1} \lambda_{2}} g_{\mathbf{k}, \mathbf{q}}^{\lambda_{1} \lambda_{2} j *} Q_{\mathbf{k}, \mathbf{q}}^{\lambda \lambda_{1} \lambda_{2} \lambda^{\prime} j} \delta\left(\varepsilon_{\mathbf{k}}^{\lambda_{1}}-\varepsilon_{\mathbf{k}-\mathbf{q}}^{\lambda_{1}}-\hbar \omega_{\mathbf{q}}^{j}\right) \\
& T_{\mathbf{k}, \mathbf{q}}^{\lambda \lambda^{\prime} j}=-i \pi \sum_{\lambda_{1} \lambda_{2}} g_{\mathbf{k}, \mathbf{q}}^{\lambda_{1} \lambda_{2} j} Q_{\mathbf{k}, \mathbf{q}}^{\lambda_{1} \lambda^{\prime} \lambda \lambda_{2} j} \delta\left(\varepsilon_{\mathbf{k}}^{\lambda_{1}}-\varepsilon_{\mathbf{k}-\mathbf{q}}^{\lambda_{2}}-\hbar \omega_{\mathbf{q}}^{j}\right) .
\end{aligned}
$$

Inserting the analytic solution of the phonon-assisted quantities into the Eq. C.20 the density matrix element yields

$$
\begin{aligned}
\dot{\sigma}_{\mathbf{k}}^{\lambda \lambda^{\prime}}=\frac{\pi}{\hbar} \sum_{\lambda_{1} \lambda_{2} \lambda_{3}} \sum_{\mathbf{q} j} & {\left[g_{\mathbf{k}, \mathbf{q}}^{\lambda^{\prime} \lambda_{1} j} g_{\mathbf{k}, \mathbf{q}}^{\lambda_{2} \lambda_{3} j *} Q_{\mathbf{k}, \mathbf{q}}^{\lambda \lambda_{2} \lambda_{3} \lambda_{1} j} \delta\left(\varepsilon_{\mathbf{k}}^{\lambda_{2}}-\varepsilon_{\mathbf{k}-\mathbf{q}}^{\lambda_{3}}-\hbar \omega_{\mathbf{q}}^{j}\right)\right.} \\
& +g_{\mathbf{k}, \mathbf{q}}^{\lambda \lambda_{1} j *} g_{\mathbf{k}, \mathbf{q}}^{\lambda_{2} \lambda_{3} j} Q_{\mathbf{k}, \mathbf{q}}^{\lambda_{2} \lambda^{\prime} \lambda_{1} \lambda_{3} j} \delta\left(\varepsilon_{\mathbf{k}}^{\lambda_{2}}-\varepsilon_{\mathbf{k}-\mathbf{q}}^{\lambda_{3}}-\hbar \omega_{\mathbf{q}}^{j}\right) \\
& -g_{\mathbf{k}+\mathbf{q}, \mathbf{q}}^{\lambda_{1} \lambda j} g_{\mathbf{k}+\mathbf{q}, \mathbf{q}}^{\lambda_{2} \lambda_{3} j *} Q_{\mathbf{k}+\mathbf{q}, \mathbf{q}}^{\lambda_{1} \lambda_{2} \lambda_{3} \lambda^{\prime} j} \delta\left(\varepsilon_{\mathbf{k}+\mathbf{q}}^{\lambda_{2}}-\varepsilon_{\mathbf{k}}^{\lambda_{3}}-\hbar \omega_{\mathbf{q}}^{j}\right) \\
& \left.-g_{\mathbf{k}+\mathbf{q}, \mathbf{q}}^{\lambda_{1} \lambda^{\prime} j} g_{\mathbf{k}+\mathbf{q}, \mathbf{q}}^{\lambda_{2} \lambda_{3} j} Q_{\mathbf{k}+\mathbf{q}, \mathbf{q}}^{\lambda_{2} \lambda_{1} \lambda \lambda^{\prime} j} \delta\left(\varepsilon_{\mathbf{k}+\mathbf{q}}^{\lambda_{2}}-\varepsilon_{\mathbf{k}}^{\lambda_{3}}-\hbar \omega_{\mathbf{q}}^{j}\right)\right] .
\end{aligned}
$$

This expression leads to the phonon-induced temporal evolution of the microscopic polarization and carrier occupation, which will be derived in the following. 
Carrier Occupation In analogy to the Coulomb-induced scattering rates, the carrier occupation can be obtained by collecting all terms in Eq. C.25 containing only diagonal matrix elements with $\sigma_{\mathbf{k}}^{\lambda \lambda^{\prime}}=\sigma_{\lambda \lambda^{\prime}} \delta_{\lambda \lambda^{\prime}}$. The resulting Boltzmann-like scattering equation reads

$$
\left.\frac{d}{d t} \rho_{\mathbf{k}}^{\lambda}\right|_{H_{c, p}}=\Gamma_{\lambda, \mathbf{k}}^{\mathrm{in}, c p}\left[1-\rho_{\mathbf{k}}^{\lambda}\right]-\Gamma_{\lambda, \mathbf{k}}^{\mathrm{out}, c p} \rho_{\mathbf{k}}^{\lambda}
$$

with the in- and out-scattering rates

$$
\begin{aligned}
& \Gamma_{\lambda, \mathbf{k}}^{\mathrm{in}, c p}=\frac{2 \pi}{\hbar} \sum_{\lambda^{\prime} j \mathbf{q}}\left[\left|g_{\mathbf{k}, \mathbf{q}}^{\lambda^{\prime} \lambda j}\right|^{2} \rho_{\mathbf{k}-\mathbf{q}}^{\lambda^{\prime}} n_{\mathbf{q}}^{j} \delta\left(\Delta \varepsilon_{\mathbf{k}, \mathbf{q}}^{\lambda \lambda^{\prime}}\right)\right. \\
& \left.+\left|g_{\mathbf{k}+\mathbf{q}, \mathbf{q}}^{\lambda^{\prime} \lambda j}\right|^{2} \rho_{\mathbf{k}+\mathbf{q}}^{\lambda^{\prime}}\left(n_{\mathbf{q}}^{j}+1\right) \delta\left(\Delta \varepsilon_{\mathbf{k}+\mathbf{q}, \mathbf{q}}^{\lambda^{\prime} \lambda}\right)\right], \\
& \Gamma_{\lambda, \mathbf{k}}^{\text {out }, c p}=\frac{2 \pi}{\hbar} \sum_{\lambda^{\prime} j \mathbf{q}}\left[\left|g_{\mathbf{k}, \mathbf{q}}^{\lambda^{\prime} \lambda j}\right|^{2}\left[1-\rho_{\mathbf{k}-\mathbf{q}}^{\lambda^{\prime}}\right]\left[n_{\mathbf{q}}^{j}+1\right] \delta\left(\Delta \varepsilon_{\mathbf{k}, \mathbf{q}}^{\lambda \lambda^{\prime}}\right)\right. \\
& \left.+\left|g_{\mathbf{k}+\mathbf{q}, \mathbf{q}}^{\lambda^{\prime} \lambda j}\right|^{2}\left[1-\rho_{\mathbf{k}+\mathbf{q}}^{\lambda^{\prime}}\right]\left(n_{\mathbf{q}}^{j}+1\right) \delta\left(\Delta \varepsilon_{\mathbf{k}+\mathbf{q}, \mathbf{q}}^{\lambda^{\prime} \lambda}\right)\right]
\end{aligned}
$$

with $\Delta \varepsilon_{\mathbf{k}, \mathbf{q}}^{\lambda \lambda^{\prime}}=\varepsilon_{\mathbf{k}}^{\lambda}-\varepsilon_{\mathbf{k}-\mathbf{q}}^{\lambda^{\prime}}-\hbar \omega_{\mathbf{q}}^{j}$.

Polarization The phonon-induced dynamics of the microscopic polarization $p_{\mathbf{k}}(t)$ is given by

$$
\left.\frac{d}{d t} p_{\mathbf{k}}(t)\right|_{H_{c, p}}=-\gamma_{\mathbf{k}}^{c p} p_{\mathbf{k}}+\mathcal{U}_{\mathbf{k}}^{c p}
$$

where the diagonal dephasing $\gamma_{\mathbf{k}}$ read

$$
\gamma_{\mathbf{k}}^{c p}=\frac{1}{2} \sum_{\lambda}\left[\Gamma_{\lambda, \mathbf{k}}^{\mathrm{in}, c p}(t)+\Gamma_{\lambda, \mathbf{k}}^{\mathrm{out}, c p}(t)\right],
$$

and the phonon off-diagonal contribution $\mathcal{U}_{\mathbf{k}}^{c p}$ is given by

$$
\begin{array}{r}
\mathcal{U}_{\mathbf{k}}^{c p}=\frac{\pi}{\hbar} \sum_{\lambda j \mathbf{q}}\left[\left|g_{\mathbf{k}+\mathbf{q}, \mathbf{q}}^{\lambda \lambda j}\right|^{2}\left[\left(1-\rho_{\mathbf{k}}^{\lambda}\right)\left(n_{\mathbf{q}}^{j}+1\right)+\rho_{\mathbf{k}}^{\lambda} n_{\mathbf{q}}^{j}\right] p_{\mathbf{k}+\mathbf{q}} \delta_{c p}^{+}\right. \\
\left.+\left|g_{\mathbf{k}, \mathbf{q}}^{\lambda \lambda j}\right|^{2}\left[\left(1-\rho_{\mathbf{k}}^{\lambda}\right) n_{\mathbf{q}}^{j}+\rho_{\mathbf{k}}^{\lambda}\left(n_{\mathbf{q}}^{j}+1\right)\right] p_{\mathbf{k}-\mathbf{q}} \delta_{c p}^{-}\right]
\end{array}
$$

with $\delta_{c p}^{ \pm}=\delta\left( \pm \varepsilon_{\mathbf{k} \pm \mathbf{q}}^{\lambda} \mp \varepsilon_{\mathbf{k}}^{\lambda}-\hbar \omega_{\mathbf{q}}^{j}\right)$ 


\section{C.2.1. Phonon dynamics}

The equation of motion of the phonon occupation is determined by the phononassisted quantities and reads

$$
\left.i \hbar \frac{d}{d t} n_{\mathbf{q}}^{j}\right|_{H_{c, p}}=-\sum_{\lambda \lambda^{\prime} \mathbf{k}}\left(g_{\mathbf{k}, \mathbf{q}}^{\lambda \lambda^{\prime} j} S_{\mathbf{k}, \mathbf{q}}^{\lambda \lambda^{\prime} j}-g_{\mathbf{k}+\mathbf{q}, \mathbf{q}}^{\lambda \lambda^{\prime} j *} T_{\mathbf{k}, \mathbf{q}}^{\lambda \lambda^{\prime} j}\right) .
$$

Applying the Markov approximation, the temporal evolution of $n_{\mathbf{q}}^{j}$ is given by

$$
\left.\dot{n}_{\mathbf{q}}^{j}(t)\right|_{\text {scat }}=\Gamma_{\mathbf{q}, j}^{\mathrm{em}}(t)\left[n_{\mathbf{q}}^{j}(t)+1\right]-\Gamma_{\mathbf{q}}^{\mathrm{abs}, j}(t) n_{\mathbf{q}}^{j}(t),
$$

with the phonon emission and absorption rates

$$
\begin{aligned}
& \Gamma_{\mathbf{q}}^{\mathrm{em}, j}(t)=\frac{2 \pi}{\hbar} \sum_{\lambda \lambda^{\prime} \mathbf{k}}\left|g_{\mathbf{k}, \mathbf{q}}^{\lambda \lambda^{\prime} j}\right|^{2} \rho_{\mathbf{k}}^{\lambda}\left[1-\rho_{\mathbf{k}-\mathbf{q}}^{\lambda^{\prime}}\right] \Delta \varepsilon_{\mathbf{k}, \mathbf{q}}^{\lambda \lambda^{\prime}}, \\
& \Gamma_{\mathbf{q}}^{\mathrm{abs}, j}(t)=\frac{2 \pi}{\hbar} \sum_{\lambda \lambda^{\prime} \mathbf{k}}\left|g_{\mathbf{k}, \mathbf{q}}^{\lambda \lambda^{\prime} j}\right|^{2}\left[1-\rho_{\mathbf{k}}^{\lambda}\right] \rho_{\mathbf{k}-\mathbf{q}}^{\lambda^{\prime}} \Delta \varepsilon_{\mathbf{k}, \mathbf{q}}^{\lambda \lambda^{\prime}}
\end{aligned}
$$




\section{Derivation of the dephasing in the dynamical screening}

In order to obtain an expression for the dephasing $\delta_{\mathbf{k}, \mathbf{q}}^{\lambda}$ in the dynamical screening (Eq. 2.47), the dynamic of the electronic density matrix element $\sigma_{12}=\left\langle a_{1}^{\dagger} a_{2}\right\rangle$ with the index $1=(\mathbf{k}, \lambda)$ is needed, which is given within the correlation expansion:

$$
\begin{aligned}
\left.i \hbar \frac{d}{d t} \sigma_{12}\right|_{H_{0}+H_{c, c}}= & \left(\varepsilon_{2}-\varepsilon_{1}\right) \sigma_{12}+\sum_{B C D} V_{C D}^{2 B}\left[\sigma_{1 C} \sigma_{B D}-\sigma_{B C} \sigma_{1 D}+S_{1 B D C}^{c}\right] \\
& -\left[\sum_{B C D} V_{C D}^{1 B}\left[\sigma_{B D} \sigma_{2 C}-\sigma_{B C} \sigma_{2 D}+S_{2 B D C}^{c}\right]\right]^{*}
\end{aligned}
$$

Here, $S_{1234}^{c}=\left\langle a_{1}^{\dagger} a_{2}^{\dagger} a_{3} a_{4}\right\rangle$ is two-particle correlation quantity stemming from the factorization $S_{1234}=\sigma_{23} \sigma_{14}-\sigma_{13} \sigma_{24}+S_{1234}^{c}$. The equation of motion of the correlation quantity $S_{1234}^{c}$ reads

$i \hbar \frac{d}{d t} S_{1234}^{c}=\left(\varepsilon_{3}+\varepsilon_{4}-\varepsilon_{1}-\varepsilon_{1}\right) S_{1234}^{c}+\sum_{A B C D} \tilde{V}_{C D}^{A B}\left[\sigma_{1 D} \sigma_{2 C} \sigma_{A 3}^{+} \sigma_{B 4}^{+}-\sigma_{A 3} \sigma_{B 4} \sigma_{1 D}^{+} \sigma_{2 C}^{+}\right]$

with the Coulomb matrix element $\tilde{V}_{C D}^{A B}=V_{C D}^{A B}-V_{D C}^{A B}$. The fist part of this equation corresponds to the free-particle contribution including the energy difference of the four involved states, whereas the second part is responsible for the screening. In order to analyze the dynamical screening formula, the definition of the electron charge density fluctuation is used, exemplary shown for the conduction band $(\lambda=$ c) [51]:

$$
\left\langle\tilde{\rho}_{\mathbf{q}}\right\rangle=-\frac{|e|}{L^{3}} \sum_{\mathbf{k}}\left\langle a_{\mathbf{k}-\mathbf{q}}^{\dagger} a_{\mathbf{k}}\right\rangle=-\frac{|e|}{L^{3}} \sum_{\mathbf{k}} \sigma_{\mathbf{k}-\mathbf{q}, \mathbf{k}} .
$$

The equation of motion for this quantity reads 51

$$
\frac{d}{d t} \sigma_{\mathbf{k}-\mathbf{q}, \mathbf{k}}=\frac{i}{\hbar}\left(\varepsilon_{\mathbf{k}-\mathbf{q}}-\varepsilon_{\mathbf{k}}\right) \sigma_{\mathbf{k}-\mathbf{q}, \mathbf{k}}+\frac{i V_{\mathbf{q}}}{\hbar}\left(\rho_{\mathbf{k}}-\rho_{\mathbf{k}-\mathbf{q}}\right) \sum_{\mathbf{k}^{\prime}} \sigma_{\mathbf{k}^{\prime}-\mathbf{q}, \mathbf{k}^{\prime}}
$$


With the ansatz $\sigma_{\mathbf{k}-\mathbf{q}, \mathbf{k}}(t)=e^{-i(\omega+i \delta) t} \sigma_{\mathbf{k}-\mathbf{q}, \mathbf{k}}(0)$ the inhomogeneous polarization function can be obtained, which leads to the dynamical screening formula with a constant dephasing $\delta_{\mathbf{k}, \mathbf{q}}=\delta$, cf. Eq. 2.47 |51|. To obtain a momentum-dependent $\delta$, we write the non-coherent equation of motion for the electron charge density in second-order Born-Markov approximation:

$$
\begin{aligned}
\left.\frac{d}{d t} \sigma_{\mathbf{k}-\mathbf{q}, \mathbf{k}}\right|_{n c}= & \frac{\pi}{\hbar} \sum_{A B C D} \tilde{V}_{C D}^{A B} \delta\left(\varepsilon_{D}+\varepsilon_{C}-\varepsilon_{B}-\varepsilon_{A}\right) \times \\
& {\left[-\sum_{b c d} V_{c d}^{\mathbf{k} b}\left(\sigma_{\mathbf{k}-\mathbf{q}, D} \sigma_{b C} \sigma_{A d}^{\dagger} \sigma_{B c}^{\dagger}-\sigma_{A d} \sigma_{B c} \sigma_{\mathbf{k}-\mathbf{q}, D}^{\dagger} \sigma_{b C}^{\dagger}\right)\right.} \\
& \left.+\sum_{a b d} V_{\mathbf{k}-\mathbf{q} d}^{a b}\left(\sigma_{a D} \sigma_{b C} \sigma_{A d}^{\dagger} \sigma_{B \mathbf{k}}^{+}-\sigma_{A d} \sigma_{B \mathbf{k}} \sigma_{a D}^{\dagger} \sigma_{b C}^{\dagger}\right)\right]
\end{aligned}
$$

This equation contains the momentum-dependent dephasing term, which is given by $\left.\frac{d}{d t} \sigma_{\mathbf{k}-\mathbf{q}, \mathbf{k}}\right|_{n c} \propto \delta_{\mathbf{k}, \mathbf{q}} \sigma_{\mathbf{k}-\mathbf{q}, \mathbf{k}}$. Neglecting all polarization terms $\sigma_{a b} \rightarrow \rho_{a}$, the dephasing $\delta_{\mathbf{k}, \mathbf{q}}$ yields

$$
\delta_{\mathbf{k}, \mathbf{q}}^{\lambda}=S_{\mathbf{k}, \mathbf{q}}^{\mathrm{in}, \lambda}+S_{\mathbf{k}, \mathbf{q}}^{\mathrm{out}, \lambda}
$$

with

$$
\begin{aligned}
& S_{\mathbf{k}, \mathbf{q}}^{\mathrm{in}, \lambda}=\frac{\pi}{\hbar} \sum_{\mathbf{a b c}}\left[V_{\mathbf{k}_{a} \lambda_{a} \mathbf{k}_{b} \lambda_{b}}^{\mathbf{k} \lambda \mathbf{k}_{c} \lambda_{c}} \tilde{V}_{\mathbf{k}}^{\mathbf{k}_{a} \lambda_{a} \mathbf{k}_{b} \lambda_{b}}{ }_{\mathbf{k}_{c} \lambda_{c}}+V_{\mathbf{k}_{a} \lambda_{a} \mathbf{k}_{b} \lambda_{b}}^{\mathbf{k}-\mathbf{q} \mathbf{k}_{c} \lambda_{c}} \tilde{V}_{\mathbf{k}-\mathbf{q} \lambda \mathbf{k}_{c} \lambda_{c}}^{\mathbf{k}_{a} \lambda_{a} \mathbf{k}_{b} \lambda_{b}}\right] \times \\
& {\left[1-\rho_{\mathbf{a}}\right] \rho_{\mathbf{b}} \rho_{\mathbf{c}} \delta\left(\varepsilon_{\mathbf{k}}^{\lambda}+\varepsilon_{\mathbf{a}}+\varepsilon_{\mathbf{b}}+\varepsilon_{\mathbf{c}}\right)} \\
& S_{\mathbf{k}, \mathbf{q}}^{\text {out }, \lambda}=\frac{\pi}{\hbar} \sum_{\mathbf{a b c}}\left[V_{\mathbf{k}_{a} \lambda_{a} \mathbf{k}_{b} \lambda_{b}}^{\mathbf{k} \lambda \mathbf{k}_{c} \lambda_{c}} \tilde{V}_{\mathbf{k}}^{\mathbf{k}_{a} \lambda_{a} \mathbf{k}_{b} \lambda_{b}}{ }_{\mathbf{k}_{c} \lambda_{c}}+V_{\mathbf{k}_{a} \lambda_{a} \mathbf{k}_{b} \lambda_{b}}^{\mathbf{k}-\mathbf{q} \lambda \mathbf{k}_{c} \lambda_{c}} \tilde{V}_{\mathbf{k}-\mathbf{q} \lambda \mathbf{k}_{c} \lambda_{c}}^{\mathbf{k}_{a} \lambda_{a} \mathbf{k}_{b} \lambda_{b}}\right] \times \\
& \rho_{\mathbf{a}}\left[1-\rho_{\mathbf{b}}\right]\left[1-\rho_{\mathbf{c}}\right] \delta\left(\varepsilon_{\mathbf{k}}^{\lambda}+\varepsilon_{\mathbf{a}}+\varepsilon_{\mathbf{b}}+\varepsilon_{\mathbf{c}}\right) .
\end{aligned}
$$

Note, that $V_{34}^{12}$ contains here the unscreened Coulomb potential.

Static Screening The static screening is evaluated at $\omega=0$ and $\delta_{\mathbf{k}, \mathbf{q}}^{\lambda}=0$. Including the wave function from 2.10 and assuming that the system is in equilibrium at $T=0$ K, i.e. $\rho_{\mathbf{k}}^{v}=1$ and $\rho_{\mathbf{k}}^{c}=0$, the static limit of the Lindhart formula (Eq. 2.47 yields for graphene

$\varepsilon(\mathbf{q})=1-\frac{2 V_{q}}{\gamma_{0}}\left[\left(\frac{q a_{B}}{Z_{\text {eff }}}\right)^{2}+1\right]^{-6} \sum_{\mathbf{k}} \frac{1}{|e(\mathbf{k}-\mathbf{q})|+|e(\mathbf{k})|}\left(1+\operatorname{Re}\left[\frac{e^{*}(\mathbf{k}) e(\mathbf{k}-\mathbf{q})}{|e(\mathbf{k})||e(\mathbf{k}-\mathbf{q})|}\right]\right)$. 
The static screening for bilayer graphene within the limit of linear optics is given by

$$
\begin{aligned}
\varepsilon(\mathbf{q})^{B G}=1 & -4 V_{q}\left[\left(\frac{q a_{B}}{Z_{\mathrm{eff}}}\right)^{2}+1\right]^{-6} \\
\sum_{\mathbf{k}} & {\left[\left(\frac{1}{\beta_{\mathbf{k}}^{\sigma_{1}} \beta_{\mathbf{k}-\mathbf{q}}^{\sigma_{1}} \Delta \varepsilon_{\mathbf{k}-\mathbf{q}, \mathbf{k}}^{v_{1} c_{1}}}+\frac{1}{\beta_{\mathbf{k}}^{\sigma_{2}} \beta_{\mathbf{k}-\mathbf{q}}^{\sigma_{2}} \Delta \varepsilon_{\mathbf{k}-\mathbf{q}, \mathbf{k}}^{v_{2} c_{2}}}\right) \cdot\left(1-\operatorname{Re}\left[\frac{e^{*}(\mathbf{k}) e(\mathbf{k}-\mathbf{q})}{|e(\mathbf{k})||e(\mathbf{k}-\mathbf{q})|}\right]\right)\right.} \\
+ & \left.\frac{1}{\beta_{\mathbf{k}}^{\sigma_{2}} \beta_{\mathbf{k}-\mathbf{q}}^{\sigma_{1}} \Delta \varepsilon_{\mathbf{k}-\mathbf{q}, \mathbf{k}}^{v_{1} c_{2}}}\left|\frac{\left(\gamma_{1}+\zeta_{\mathbf{k}}\right)\left(\gamma_{1}-\zeta_{\mathbf{k}-\mathbf{q}}\right)}{2 \gamma_{0} e(\mathbf{k}) e(\mathbf{k}-\mathbf{q})}+\frac{e(\mathbf{k}) e^{*}(\mathbf{k}-\mathbf{q})}{e^{*}(\mathbf{k}) e(\mathbf{k}-\mathbf{q})}+1\right|^{2}\right]
\end{aligned}
$$

with $\beta_{\mathbf{k}}^{\sigma_{i}}=\left(4-\frac{4 \gamma_{1}}{\left(\gamma_{1}+\sigma_{i} \sqrt{4|e(\mathbf{k})|^{2} \gamma_{0}{ }^{2}+\gamma_{1}^{2}}\right)}\right)^{\frac{1}{2}}$, where $\sigma_{i}=+1(-1)$ for $i=1(i=2)$. Furthermore the abbreviations $\Delta \varepsilon_{\mathbf{k}-\mathbf{q}, \mathbf{k}}^{\lambda \lambda^{\prime}}=\varepsilon_{\mathbf{k}-\mathbf{q}}^{\lambda}-\varepsilon_{\mathbf{k}}^{\lambda^{\prime}}$ and $\zeta_{\mathbf{k}}=\sqrt{4|e(\mathbf{k})|^{2} \gamma_{0}{ }^{2}+\gamma_{1}^{2}}$ are used. 


\section{E. Perturbation approach of the phonon-assisted quantities}

Here, the field-assisted second order contribution, responsible for the phononassisted intraband absorption, is evaluated. In addition, the equation of motion for the occupation and polarization including the intraband absorption processes is determined. The equation of motion for the phonon-assisted quantity $S_{\mathbf{k}, \mathbf{q}}^{\lambda_{1} \lambda_{2} j}$ $\left(\tilde{S}_{\mathbf{k}, \mathbf{q}}^{\lambda_{1} \lambda_{2} j}\right.$ in analogy) reads

$$
\begin{gathered}
\dot{S}_{\mathbf{k}, \mathbf{q}}^{\lambda_{1} \lambda_{2} j}=\frac{i}{\hbar} \Delta \varepsilon_{\mathbf{k}, \mathbf{q}}^{\lambda_{1} \lambda_{2}} S_{\mathbf{k}, \mathbf{q}}^{\lambda_{1} \lambda_{2} j}+\frac{i}{\hbar} \sum_{\lambda_{3} \lambda_{4}} g_{\mathbf{k}, \mathbf{q}}^{\lambda_{3} \lambda_{4} j *} Q_{\mathbf{k}, \mathbf{q}}^{\lambda_{1} \lambda_{3} \lambda_{4} \lambda_{2} j} \\
+\frac{\hbar e_{0}}{m_{0}} \sum_{\lambda^{\prime}}\left[S_{\mathbf{k}, \mathbf{q}}^{\lambda_{1} \lambda^{\prime} j} \mathbf{M}_{\mathbf{k}-\mathbf{q}}^{\lambda_{2} \lambda^{\prime}}-S_{\mathbf{k}, \mathbf{q}}^{\lambda^{\prime} \lambda_{2} j} \mathbf{M}_{\mathbf{k}}^{\lambda^{\prime} \lambda_{1}}\right] \cdot \mathbf{A}(t),
\end{gathered}
$$

where the vector potential is assumed to be linear polarized. To be able to solve $S_{\mathbf{k}, \mathbf{q}}^{\lambda_{1} \lambda_{2} j}$ analytically, a perturbation approach is used (exemplary shown for $S_{\mathbf{k}, \mathbf{q}}^{c c, j}$ ):

$$
S_{\mathbf{k}, \mathbf{q}}^{c c, j}=S_{\mathbf{k , \mathbf { q } ( 0 )}}^{c c, j}+\epsilon S_{\mathbf{k}, \mathbf{q}(0)}^{c c, j},
$$

where $\epsilon=A_{0}$. Inserting this ansatz into Eq. E.1, the zeroth and second order of $\dot{S}_{\mathbf{k}, \mathbf{q}}^{c c, j} \operatorname{read}$

$$
\begin{aligned}
& \dot{S}_{\mathbf{k}, \mathbf{q}(0)}^{c c, j}=\frac{i}{\hbar} \Delta \varepsilon_{\mathbf{k}, \mathbf{q}}^{c c} S_{\mathbf{k}, \mathbf{q}(0)}^{c c, j}+\frac{i}{\hbar} \sum_{\lambda_{3} \lambda_{4}} g_{\mathbf{k}, \mathbf{q}}^{\lambda_{3} \lambda_{4} j *} Q_{\mathbf{k}, \mathbf{q}}^{c \lambda_{3} \lambda_{4} c, j} \\
& \dot{S}_{\mathbf{k}, \mathbf{q}(1)}^{c c, j}=\frac{i}{\hbar} \Delta \varepsilon_{\mathbf{k}, \mathbf{q}}^{c c} S_{\mathbf{k}, \mathbf{q}(1)}^{c c, j)}+\frac{\hbar e_{0}}{m_{0}} \sum_{\lambda^{\prime}}\left[\left(M_{\mathbf{k}-\mathbf{q}}^{c \lambda^{\prime}} S_{\mathbf{k}, \mathbf{q}(0)}^{c \lambda^{\prime}, j}-M_{\mathbf{k}}^{\lambda^{\prime} c} S_{\mathbf{k}, \mathbf{q}(0)}^{\lambda^{\prime} c, j}\right) \tilde{A}(t)\right],
\end{aligned}
$$

where $\tilde{A}(t)=A(t) / A_{0}$. The stationary limit of $S_{\mathbf{k}, \mathbf{q},(0)}^{c c, j}$ yields

$$
S_{\mathbf{k}, \mathbf{q}, l i m}^{c c j(0)}=-\frac{\sum_{\lambda_{3} \lambda_{4}} g_{\mathbf{k}, \mathbf{q}}^{\lambda_{3} \lambda_{4} j *} Q_{\mathbf{k}, \mathbf{q}}^{c \lambda_{3} \lambda_{4} c, j}}{\Delta \varepsilon_{\mathbf{k}, \mathbf{q}}^{c c}}
$$


Inserting for the first perturbation order of $\dot{S}_{\mathbf{k}, \mathbf{q}}^{c c j}$ the stationary limit, the following equation is obtained:

$$
\begin{aligned}
\dot{S}_{\mathbf{k}, \mathbf{q}(1)}^{c c, j}= & \frac{i}{\hbar} \Delta \varepsilon_{\mathbf{k}, \mathbf{q}}^{c c} S_{\mathbf{k}, \mathbf{q}(1)}^{c c, j} \\
& -\frac{\hbar e_{0}}{m_{0}} \sum_{\lambda_{3} \lambda_{4}} g_{\mathbf{k}, \mathbf{q}}^{\lambda_{3} \lambda_{4} j *}\left[\Delta M_{\mathbf{k}, \mathbf{q}}^{c c} \frac{Q_{\mathbf{k}, \mathbf{q}}^{c \lambda_{3} \lambda_{4} c, j}}{\Delta \varepsilon_{\mathbf{k}, \mathbf{q}}^{c c}}+M_{\mathbf{k}-\mathbf{q}}^{c v} \frac{Q_{\mathbf{k}, \mathbf{q}}^{c \lambda_{3} \lambda_{4} v, j}}{\Delta \varepsilon_{\mathbf{k}, \mathbf{q}}^{c v}}+M_{\mathbf{k}}^{v c} \frac{Q_{\mathbf{k}, \mathbf{q}}^{v \lambda_{3} \lambda_{4} c, j}}{\Delta \varepsilon_{\mathbf{k}, \mathbf{q}}^{v c}}\right] \tilde{A}(t),
\end{aligned}
$$

where $\Delta M_{\mathbf{k}, \mathbf{q}}^{c c}=M_{\mathbf{k}-\mathbf{q}}^{c c}-M_{\mathbf{k}}^{c c}$. The solution of $\dot{S}_{\mathbf{k}, \mathbf{q}(1)}^{c c j}$ within the Markov approximation (exemplary shown for the first term proportional to $\Delta M_{\mathbf{k}, \mathbf{q}}^{c c}$ ) reads

$$
\begin{aligned}
S_{\mathbf{k}, \mathbf{q}(1)}^{c c, j}= & -\frac{\hbar e_{0}}{m_{0}} \frac{\sum_{\lambda_{3} \lambda_{4}} g_{\mathbf{k}, \mathbf{q}}^{\lambda_{3} \lambda_{4} j *}}{\Delta \varepsilon_{\mathbf{k}, \mathbf{q}}^{c c}} \int_{-\infty}^{t} e^{\left(\frac{i}{\hbar} \Delta \varepsilon_{\mathbf{k}, \mathbf{q}}^{c c}-\gamma\right)\left(t-t^{\prime}\right)} A\left(t^{\prime}\right) Q_{\mathbf{k}, \mathbf{q}}^{c \lambda_{3} \lambda_{4} c, j}\left(t^{\prime}\right) d t^{\prime} \\
= & -\frac{\hbar e_{0}}{m_{0}} \frac{\sum_{\lambda_{3} \lambda_{4}} g_{\mathbf{k}, \mathbf{q}}^{\lambda_{3} \lambda_{4} j *}}{\Delta \varepsilon_{\mathbf{k}, \mathbf{q}}^{c c}} \int_{0}^{\infty} e^{\left(\frac{i}{\hbar} \Delta \varepsilon_{\mathbf{k}, \mathbf{q}}^{c c}-\gamma\right) t^{\prime}} A\left(t-t^{\prime}\right) Q_{\mathbf{k}, \mathbf{q}}^{c \lambda_{3} \lambda_{4} c, j}\left(t-t^{\prime}\right) d t^{\prime}, \\
= & -\frac{\hbar e_{0}}{m_{0}} \frac{\sum_{\lambda_{3} \lambda_{4}} g_{\mathbf{k}, \mathbf{q}}^{\lambda_{3} \lambda_{4} j *}}{2 \Delta \varepsilon_{\mathbf{k}, \mathbf{q}}^{c c}} Q_{\mathbf{k}, \mathbf{q}}^{c \lambda_{3} \lambda_{4} c, j}(t) \hat{A}(t) \times \\
& {\left[\frac{e^{-i \omega_{L} t}}{-\frac{i}{\hbar}\left(\Delta \varepsilon_{\mathbf{k}, \mathbf{q}}^{\lambda_{3} \lambda_{4}}+\hbar \omega_{L}\right)+\gamma}+\frac{e^{i \omega_{L} t}}{-\frac{i}{\hbar}\left(\Delta \varepsilon_{\mathbf{k}, \mathbf{q}}^{\lambda_{3} \lambda_{4}}-\hbar \omega_{L}\right)+\gamma}\right] . }
\end{aligned}
$$

Note, that the assumptions $\tilde{A}\left(t-t^{\prime}\right) \approx \hat{A}(t) \cos \left(\omega_{L}\left(t-t^{\prime}\right)\right)$ and $Q_{\mathbf{k}, \mathbf{q}}^{c \lambda_{3} \lambda_{4} c, j}\left(t-t^{\prime}\right) \approx$ $Q^{c \lambda_{3} \lambda_{4} c, j}(t) e^{-\frac{i}{\hbar}\left(\varepsilon_{\mathbf{k}}^{c}-\varepsilon_{\mathbf{k}}^{\lambda_{3}}+\varepsilon_{\mathbf{k}-\mathbf{q}}^{\lambda_{4}}-\varepsilon_{\mathbf{k}-\mathbf{q}}^{c}\right) t^{\prime}}$ are used. The vector potential $\tilde{A}\left(t-t^{\prime}\right)$ and the scattering kernel $Q_{\mathbf{k}, \mathbf{q}}^{c \lambda_{3} \lambda_{4} c, j}\left(t-t^{\prime}\right)$ are separated in a slow and quick component. Finally, assuming $\gamma \rightarrow 0$ the solution of the first order of the phonon-assisted quantity yields

$$
\begin{aligned}
S_{\mathbf{k}, \mathbf{q}(1)}^{c c, j}= & -\frac{\hbar e_{0}}{m_{0}} \frac{\sum_{\lambda_{3} \lambda_{4}} g_{\mathbf{k}, \mathbf{q}}^{\lambda_{3} \lambda_{4} j *}}{2 \Delta \varepsilon_{\mathbf{k}, \mathbf{q}}^{c c}} Q_{\mathbf{k}, \mathbf{q}}^{c \lambda_{3} \lambda_{4} c, j}(t) \hat{A}(t) \times \\
\pi & {\left[e^{-i \omega_{L} t} \delta\left(\Delta \varepsilon_{\mathbf{k}, \mathbf{q}}^{\lambda_{3} \lambda_{4}}+\hbar \omega_{L}\right)+\frac{i e^{-i \omega_{L} t}}{\hbar} \mathcal{P}\left(\frac{1}{\frac{1}{\hbar}\left(\Delta \varepsilon_{\mathbf{k}, \mathbf{q}}^{\lambda_{3} \lambda_{4}}+\hbar \omega_{L}\right)}\right)\right.} \\
& \left.+e^{i \omega_{L} t} \delta\left(\Delta \varepsilon_{\mathbf{k}, \mathbf{q}}^{\lambda_{3} \lambda_{4}}-\hbar \omega_{L}\right)+\frac{i e^{i \omega_{L} t}}{\hbar} \mathcal{P}\left(\frac{1}{\frac{1}{\hbar}\left(\Delta \varepsilon_{\mathbf{k}, \mathbf{q}}^{\lambda_{3} \lambda_{4}}-\hbar \omega_{L}\right)}\right)\right]
\end{aligned}
$$

where $P($.$) denotes the principal value, which will be neglected. Inserting all$ expressed phonon-assisted quantities in Eq. E.2, all terms proportional to the offdiagonal matrix element $\mathbf{M}_{\mathbf{k}}^{v c}$ turn out to cancel in the equation of $\rho_{\mathbf{k}}^{\lambda}$. Without 
the off-diagonal matrix element, $S_{\mathbf{k}, \mathbf{q}}^{\lambda_{1} \lambda_{2} j}$ is given by

$$
\begin{aligned}
S_{\mathbf{k}, \mathbf{q}}^{\lambda_{1} \lambda_{2} j}= & i \pi \sum_{\lambda_{3} \lambda_{4}} g_{\mathbf{k}, \mathbf{q}}^{\lambda_{3} \lambda_{4} j *} Q_{\mathbf{k}, \mathbf{q}}^{\lambda_{1} \lambda_{3} \lambda_{4} \lambda_{2} j} \delta\left(\Delta \varepsilon_{\mathbf{k}, \mathbf{q}}^{\lambda_{3} \lambda_{4}}\right) \\
& -\frac{\hbar e_{0}}{m_{0}} \frac{\sum_{\lambda_{3} \lambda_{4}} g_{\mathbf{k}, \mathbf{q}}^{\lambda_{3} \lambda_{4} j *} Q_{\mathbf{k}, \mathbf{q}}^{\lambda_{1} \lambda_{3} \lambda_{4} \lambda_{2} j}\left(M_{\mathbf{k}-\mathbf{q}}^{\lambda_{2} \lambda_{2}}-M_{\mathbf{k}}^{\lambda_{1} \lambda_{1}}\right) \cdot \tilde{A}(t) A_{0}}{2 \Delta \varepsilon_{\mathbf{k}, \mathbf{q}}^{\lambda_{1} \lambda_{2}}} \times \\
& \pi\left[e^{-i \omega_{L} t} \delta\left(\Delta \varepsilon_{\mathbf{k}, \mathbf{q}}^{\lambda_{3} \lambda_{4}}+\hbar \omega_{L}\right)+e^{i \omega_{L} t} \delta\left(\Delta \varepsilon_{\mathbf{k}, \mathbf{q}}^{\lambda_{3} \lambda_{4}}-\hbar \omega_{L}\right)\right] .
\end{aligned}
$$

The first term stems from the zeroth order of $S$, whereas the second term is the solution of the first order of $S$ responsible for the phonon-assisted absorption processes.

Occupation: Inserting the derived phonon-assisted quantities into Eq. E.5, the occupation reads

$$
\left.\dot{\rho}_{\mathbf{k}}^{\lambda}\right|_{H_{c, p}+H_{c, c}}=\left[\Gamma_{\lambda, \mathbf{k}}^{\text {in }}+\Gamma_{\lambda, \mathbf{k}}^{\text {in, A }}\right]\left(1-\rho_{\mathbf{k}}^{\lambda}\right)-\left[\Gamma_{\lambda, \mathbf{k}}^{\text {out }}+\Gamma_{\lambda, \mathbf{k}}^{\text {out }, \mathrm{A}}\right] \rho_{\mathbf{k}}^{\lambda}
$$

with the field-assisted second order contributions

$$
\begin{aligned}
& \Gamma_{\lambda, \mathbf{k}}^{\mathrm{in}, \mathrm{A}}=2 \pi \sum_{\lambda^{\prime} j \mathbf{q}}\left[\left|g_{\mathbf{k}, \mathbf{q}}^{\lambda^{\prime} \lambda j}\right|^{2}\left(\frac{\tilde{M}_{\mathbf{k}, \mathbf{q}}^{\lambda^{\prime} \lambda}}{\Delta \varepsilon_{\mathbf{k}, \mathbf{q}}^{\lambda \lambda^{\prime}}} \delta\left(\Delta \varepsilon_{\mathbf{k}, \mathbf{q}}^{\lambda \lambda^{\prime}} \pm \hbar \omega_{L}\right)\right) \rho_{\mathbf{k}-\mathbf{q}}^{\lambda^{\prime}} n_{\mathbf{q}}^{j}\right. \\
& \left.+\left|g_{\mathbf{k}+\mathbf{q}, \mathbf{q}}^{\lambda^{\prime} \lambda j}\right|^{2}\left(\frac{\tilde{M}_{\mathbf{k}+\mathbf{q}, \mathbf{q}}^{\lambda \lambda^{\prime}}}{\Delta \varepsilon_{\mathbf{k}+\mathbf{q}, \mathbf{q}}^{\lambda^{\prime} \lambda}} \delta\left(\Delta \varepsilon_{\mathbf{k}+\mathbf{q}, \mathbf{q}}^{\lambda^{\prime} \lambda} \pm \hbar \omega_{L}\right)\right) \rho_{\mathbf{k}+\mathbf{q}}^{\lambda^{\prime}}\left(n_{\mathbf{q}}^{j}+1\right)\right], \\
& \Gamma_{\lambda, \mathbf{k}}^{\text {out }, \mathrm{A}}=2 \pi \sum_{\lambda^{\prime} j \mathbf{q}}\left[\left|g_{\mathbf{k}, \mathbf{q}}^{\lambda^{\prime} \lambda j}\right|^{2}\left(\frac{\tilde{M}_{\mathbf{k}, \mathbf{q}}^{\lambda^{\prime} \lambda}}{\Delta \varepsilon_{\mathbf{k}, \mathbf{q}}^{\lambda \lambda^{\prime}}} \delta\left(\Delta \varepsilon_{\mathbf{k}, \mathbf{q}}^{\lambda \lambda^{\prime}} \pm \hbar \omega_{L}\right)\right)\left(\rho_{\mathbf{k}-\mathbf{q}}^{\lambda^{\prime}}+1\right)\left(n_{\mathbf{q}}^{j}+1\right)\right. \\
& \left.+\left|g_{\mathbf{k}+\mathbf{q}, \mathbf{q}}^{\lambda^{\prime} \lambda j}\right|^{2}\left(\frac{\tilde{M}_{\mathbf{k}+\mathbf{q}, \mathbf{q}}^{\lambda \lambda^{\prime}}}{\Delta \varepsilon_{\mathbf{k}+\mathbf{q}, \mathbf{q}}^{\lambda^{\prime} \lambda}} \delta\left(\Delta \varepsilon_{\mathbf{k}+\mathbf{q}, \mathbf{q}}^{\lambda^{\prime} \lambda} \pm \hbar \omega_{L}\right)\right)\left(\rho_{\mathbf{k}+\mathbf{q}}^{\lambda^{\prime}}+1\right) n_{\mathbf{q}}^{j}\right],
\end{aligned}
$$

where $\tilde{M}_{\mathbf{k}, \mathbf{q}}^{\lambda \lambda^{\prime}}=i e_{0} / m_{0}\left(\mathbf{M}_{\mathbf{k}-\mathbf{q}}^{\lambda^{\prime} \lambda^{\prime}}-\mathbf{M}_{\mathbf{k}}^{\lambda \lambda}\right) \cdot \mathbf{A}(t)$.

Polarization $p_{\mathbf{k}}$ : The equation of motion for the polarization is given by

$$
\dot{p}_{\mathbf{k}}=\left[i \omega_{\mathbf{k}}^{v c}-\gamma_{\mathbf{k}}\right] p_{\mathbf{k}}+i \Omega_{\mathbf{k}}^{v c}\left[\rho_{\mathbf{k}}^{v}-\rho_{\mathbf{k}}^{c}\right]+\mathcal{U}_{\mathbf{k}}^{\text {off }}+\mathcal{U}_{\mathbf{k}}^{c p, A},
$$


with the field-assisted non-diagonal element

$$
\begin{aligned}
\mathcal{U}_{\mathbf{k}}^{c p, A} & =\frac{\pi}{\hbar} \sum_{\lambda j \mathbf{q}}\left[\left|g_{\mathbf{k}, \mathbf{q}}^{\lambda \lambda j}\right|^{2}\left(\frac{\tilde{M}_{\mathbf{k}, \mathbf{q}}^{\lambda^{\prime} \lambda}}{\Delta \varepsilon_{\mathbf{k}, \mathbf{q}}^{\lambda \lambda^{\prime}}} \delta\left(\Delta \varepsilon_{\mathbf{k}, \mathbf{q}}^{\lambda \lambda} \pm \hbar \omega_{L}\right)\right) p_{\mathbf{k}-\mathbf{q}}\left[\left(1-\rho_{\mathbf{k}}^{\lambda}\right) n_{\mathbf{q}}^{j}+\rho_{\mathbf{k}}^{\lambda}\left(n_{\mathbf{q}}^{j}+1\right)\right]\right. \\
& \left.+\left|g_{\mathbf{k}+\mathbf{q}, \mathbf{q}}^{\lambda \lambda j}\right|^{2}\left(\frac{\tilde{M}_{\mathbf{k}+\mathbf{q}, \mathbf{q}}^{\lambda \lambda^{\prime}}}{\Delta \varepsilon_{\mathbf{k}+\mathbf{q}, \mathbf{q}}^{\lambda^{\prime} \lambda}} \delta\left(\Delta \varepsilon_{\mathbf{k}+\mathbf{q}, \mathbf{q}}^{\lambda \lambda} \pm \hbar \omega_{L}\right)\right) p_{\mathbf{k}+\mathbf{q}}\left[\left(1-\rho_{\mathbf{k}}^{\lambda}\right)\left(n_{\mathbf{q}}^{j}+1\right)+\rho_{\mathbf{k}}^{\lambda} n_{\mathbf{q}}\right]\right] .
\end{aligned}
$$

Note, that these terms have no contribution to the absorption, since they are proportional to $\mathbf{A}^{2}(t)$.

Occupation in the frequency space The occupation can be simplified by using the ansatz

$$
\rho_{\mathbf{k}}^{\lambda}(t)=\rho_{\mathbf{k}}^{\lambda, 0}(t)+\delta \rho_{\mathbf{k}}^{\lambda}(t)
$$

Here, $\rho_{\mathbf{k}}^{\lambda, 0}(t)$ contains the pump-induced occupation resulting from the Coulomband phonon-assisted scattering rates $\Gamma_{\lambda, \mathbf{k}}^{\text {in,out }}\left[41\right.$, while $\delta \rho_{\mathbf{k}}^{\lambda}(t)$ describes a small perturbation induced by the probe pulse. Inserting this ansatz into Eq. E.6 the occupation reads

$$
\begin{aligned}
\dot{\rho}_{\mathbf{k}}^{\lambda, 0}(t)+\delta \dot{\rho}_{\mathbf{k}}^{\lambda}(t)= & \left(\Gamma_{\lambda, \mathbf{k}}^{\mathrm{in}, 0}+\Gamma_{\lambda, \mathbf{k}}^{\mathrm{in}, A}\right)\left[1-\rho_{\mathbf{k}}^{\lambda, 0}(t)-\delta \rho_{\mathbf{k}}^{\lambda}(t)\right]- \\
& \left(\Gamma_{\lambda, \mathbf{k}}^{\text {out }, 0}+\Gamma_{\lambda, \mathbf{k}}^{\text {out }, A}\right)\left[\rho_{\mathbf{k}}^{\lambda, 0}(t)+\delta \rho_{\mathbf{k}}^{\lambda}(t)\right] .
\end{aligned}
$$

Note, that the scattering rates are also separated: $\Gamma_{\lambda, \mathbf{k}}^{\text {in,out }, 0}=\Gamma_{\lambda, \mathbf{k}}^{\text {in out }, \rho_{\mathbf{k}}^{\lambda, 0}}+\Gamma_{\lambda, \mathbf{k}}^{\text {in,out }, \delta \rho_{\mathbf{k}}^{\lambda}}$. The field-assisted contribution $\Gamma_{\lambda, \mathbf{k}}^{\mathrm{in}, \mathrm{out}, A, \delta \rho_{\mathbf{k}}^{\lambda}}$ is non-linear and will be neglected. The separation of the occupation yields

$$
\begin{aligned}
\dot{\rho}_{\mathbf{k}}^{\lambda, 0}(t)= & \Gamma_{\lambda, \mathbf{k}}^{\mathrm{in}, \rho_{\mathbf{k}}^{\lambda, 0}}\left[1-\rho_{\mathbf{k}}^{\lambda, 0}(t)\right]-\Gamma_{\lambda, \mathbf{k}}^{\text {out }, \rho_{\mathbf{k}}^{\lambda, 0}} \rho_{\mathbf{k}}^{\lambda, 0}(t), \\
\delta \dot{\rho}_{\mathbf{k}}^{\lambda}(t)= & \Gamma_{\lambda, \mathbf{k}}^{\mathrm{in}, A}\left[1-\rho_{\mathbf{k}}^{\lambda, 0}(t)\right]-\Gamma_{\lambda, \mathbf{k}}^{\text {out }, A} \rho_{\mathbf{k}}^{\lambda, 0}(t)-\left[\Gamma_{\lambda, \mathbf{k}}^{\text {in }, 0}+\Gamma_{\lambda, \mathbf{k}}^{\text {out }, 0}\right] \delta \rho_{\mathbf{k}}^{\lambda}(t)- \\
& {\left[\Gamma_{\lambda, \mathbf{k}}^{\mathrm{in}, A}+\Gamma_{\lambda, \mathbf{k}}^{\text {out }, A}\right] \delta \rho_{\mathbf{k}}^{\lambda}(t)+\Gamma_{\lambda, \mathbf{k}}^{\mathrm{in}, \delta \rho_{\mathbf{k}}^{\lambda}}\left[1-\rho_{\mathbf{k}}^{\lambda, 0}(t)\right]-\Gamma_{\lambda, \mathbf{k}}^{\text {out }, \delta \rho_{\mathbf{k}}^{\lambda}} \rho_{\mathbf{k}}^{\lambda, 0}(t) . }
\end{aligned}
$$

Assuming a constant $\tilde{\gamma}=\Gamma_{\lambda, \mathbf{k}}^{\text {in }, 0}+\Gamma_{\lambda, \mathbf{k}}^{\text {out }, 0}$ and a delta pulse $\mathbf{A}(t)=\mathbf{A}_{0} \delta(t-\tau)$ and neglecting the non-linear terms proportional to the vector potential as well as the off diagonal contribution proportional in $\Gamma_{\lambda, \mathbf{k}}^{\text {in out }, \delta \rho_{\mathbf{k}}^{\lambda}}$, the Fourier transform of $\delta \rho_{\mathbf{k}}^{\lambda}(\omega)$ is given by

$$
\delta \rho_{\mathbf{k}}^{\lambda}(\omega, \tau)=\frac{\Gamma_{\lambda, \mathbf{k}}^{\mathrm{in}, A}(\omega, \tau)\left[1-\rho_{\mathbf{k}}^{\lambda, 0}(\tau)\right]-\Gamma_{\lambda, \mathbf{k}}^{\mathrm{out}, A}(\omega, \tau) \rho_{\mathbf{k}}^{\lambda, 0}(\tau)}{i \omega+\tilde{\gamma}} e^{-i \omega \tau}
$$


Polarization in the frequency space The equation of motion for the polarization reads

$$
\dot{p}_{\mathbf{k}}=\left[i \omega_{\mathbf{k}}^{v c}-\gamma_{\mathbf{k}}\right] p_{\mathbf{k}}+i \Omega_{\mathbf{k}}^{v c}\left[\rho_{\mathbf{k}}^{v}-\rho_{\mathbf{k}}^{c}\right]+\mathcal{U}_{\mathbf{k}}^{\text {off }}+\mathcal{U}_{\mathbf{k}}^{c p, A},
$$

where $\gamma_{\mathbf{k}}=\frac{1}{2} \sum_{\lambda}\left[\Gamma_{\lambda, \mathbf{k}}^{\mathrm{in}}+\Gamma_{\lambda, \mathbf{k}}^{\text {out }}\right] \equiv \gamma$ is assumed to be constant. Neglecting the offdiagonal contribution, the polarization can be separated in a real and imaginary part:

$$
\frac{d}{d t}\left(p_{\mathbf{k}}^{\prime}+p_{\mathbf{k}}^{\prime \prime}\right)=\left[i \omega_{\mathbf{k}}^{v c}-\gamma\right]\left(p_{\mathbf{k}}^{\prime}+i p_{\mathbf{k}}^{\prime \prime}\right)-\frac{e_{0}}{m_{0}} \mathbf{A}(t) \mathbf{M}_{\mathbf{k}}^{v c}\left[\rho_{\mathbf{k}}^{v}(t)-\rho_{\mathbf{k}}^{c}(t)\right]
$$

Neglecting all non-linear terms in the vector potential, the real and imaginary part of the polarization read

$$
\begin{aligned}
& \dot{p}_{\mathbf{k}}^{\prime}(t)=-\omega_{\mathbf{k}}^{v c} p_{\mathbf{k}}^{\prime \prime}(t)-\gamma_{\mathbf{k}} p_{\mathbf{k}}^{\prime}(t)-\frac{e_{0}}{m_{0}} \mathbf{A}(t) \mathbf{M}_{\mathbf{k}}^{v c}\left[\rho_{\mathbf{k}}^{v}(t)-\rho_{\mathbf{k}}^{c}(t)\right] \\
& \dot{p}_{\mathbf{k}}^{\prime \prime}(t)=\omega_{\mathbf{k}}^{v c} p_{\mathbf{k}}^{\prime}(t)-\gamma p_{\mathbf{k}}^{\prime \prime}
\end{aligned}
$$

Assuming a delta pulse $\mathbf{A}(t)=\mathbf{A}_{0} \delta(t-\tau)$, the imaginary part $p_{\mathbf{k}}^{\prime \prime}(t)$ can be written in the frequency space as

$$
\begin{aligned}
& p_{\mathbf{k}}^{\prime \prime}(\omega, \tau)=-\frac{e_{0}}{m_{0}} \mathbf{A}_{0} \mathbf{M}_{\mathbf{k}}^{v c} \Delta \rho_{\mathbf{k}}(\tau) {\left[i \frac{2 \gamma \omega_{\mathbf{k}}^{v c} \omega}{\left(\left(\omega_{\mathbf{k}}^{v c}\right)^{2}+\gamma^{2}-\omega^{2}\right)^{2}+4 \gamma^{2} \omega^{2}}+\right.} \\
&\left.\frac{\omega_{\mathbf{k}}^{v c}\left(\left(\omega_{\mathbf{k}}^{v c}\right)^{2}+\gamma^{2}-\omega^{2}\right)}{\left(\left(\omega_{\mathbf{k}}^{v c}\right)^{2}+\gamma^{2}-\omega^{2}\right)^{2}+4 \gamma^{2} \omega^{2}}\right]
\end{aligned}
$$

where $\Delta \rho_{\mathbf{k}}(t)=\rho_{\mathbf{k}}^{v}(t)-\rho_{\mathbf{k}}^{c}(t)$.

Absorption The current density in the frequency space reads

$$
\mathbf{j}(\omega)=\frac{2 e_{0} \hbar}{m_{0} L^{2}} \sum_{\mathbf{k}}\left[2 \mathbf{M}_{\mathbf{k}}^{v c} p_{\mathbf{k}}^{\prime \prime}(\omega)-i \sum_{\lambda} \mathbf{M}_{\mathbf{k}}^{\lambda \lambda} \rho_{\mathbf{k}}^{\lambda}(\omega)-\frac{e_{0}}{\hbar} \mathbf{A}(\omega) \sum_{\lambda} \rho_{\mathbf{k}}^{t h, \lambda}\right] .
$$

It can be shown that the third term of the current in Eq. 4.11 cancels with the real part of interband term resulting in a purely imaginary current [91. With $\chi(\omega)=\frac{j(\omega)}{\varepsilon_{0} \omega^{2} A(\omega)}$ and $\alpha(\omega)=\frac{\omega}{c_{0}} \operatorname{Im}[\chi(\omega)]$ the absorption is given by

$$
\alpha(\omega)=\frac{\omega}{c_{0}} \frac{2 e_{0} \hbar}{\varepsilon_{0} \omega^{2} m_{0} L^{2}} \sum_{\mathbf{k}} \operatorname{Im}\left[\frac{2 \mathbf{M}_{\mathbf{k}}^{v c} \cdot \hat{\mathbf{e}}_{A} p_{\mathbf{k}}^{\prime \prime}(\omega)}{A(\omega)}-i \sum_{\lambda} \frac{\mathbf{M}_{\mathbf{k}}^{\lambda \lambda} \cdot \hat{\mathbf{e}}_{A} \delta \rho_{\mathbf{k}}^{\lambda}(\omega)}{A(\omega)}\right]
$$




\section{F. Terahertz dynamics}

In this appendix the electron-electron and electron-phonon contributions are determined in the $\mathrm{THz}$ regime induced by a probe pulse.

\section{F.1. Electron-Phonon Scattering}

The phonon-induced carrier occupation reads

$$
\left.\frac{d}{d t} \rho_{\mathbf{k}}^{\lambda}\right|_{H_{c, p}}=\Gamma_{\lambda, \mathbf{k}}^{\mathrm{in}, c p}\left[1-\rho_{\mathbf{k}}^{\lambda}\right]-\Gamma_{\lambda, \mathbf{k}}^{\text {out }, c p} \rho_{\mathbf{k}}^{\lambda}
$$

where the scattering rates are given by

$$
\Gamma_{\lambda, \mathbf{k}}^{\mathrm{in}, c p}=\frac{2 \pi}{\hbar} \sum_{\lambda^{\prime} j \mathbf{k}^{\prime}}\left[\left|g_{\mathbf{k}^{\prime}, \mathbf{k}^{\prime}-\mathbf{k}}^{\lambda^{\prime} \lambda j}\right|^{2} \rho_{\mathbf{k}^{\prime}}^{\lambda^{\prime}}\left[n_{\mathbf{k}^{\prime}-\mathbf{k}}^{j}+1\right] \delta\left(\Delta \varepsilon^{-}\right)+\left|g_{\mathbf{k}, \mathbf{k}-\mathbf{k}^{\prime}}^{\lambda^{\prime} \lambda j}\right|^{2} \rho_{\mathbf{k}^{\prime}}^{\lambda^{\prime}} n_{\mathbf{k}-\mathbf{k}^{\prime}}^{j} \delta\left(\Delta \varepsilon^{+}\right)\right],
$$

and

$$
\begin{aligned}
& \Gamma_{\lambda, \mathbf{k}}^{\text {out }, c p}=\frac{2 \pi}{\hbar} \sum_{\lambda^{\prime} j \mathbf{k}^{\prime}} {\left[\left|g_{\mathbf{k}^{\prime}, \mathbf{k}^{\prime}-\mathbf{k}}^{\lambda^{\prime} \lambda j}\right|^{2}\left[1-\rho_{\mathbf{k}^{\prime}}^{\lambda^{\prime}}\right] n_{\mathbf{k}^{\prime}-\mathbf{k}}^{j} \delta\left(\Delta \varepsilon^{-}\right)\right.} \\
&\left.+\left|g_{\mathbf{k}, \mathbf{k}-\left.\mathbf{k}^{\prime}\right|^{\prime} \lambda j}\right|^{2}\left[1-\rho_{\mathbf{k}^{\prime}}^{\lambda^{\prime}}\right]\left[n_{\mathbf{k}-\mathbf{k}^{\prime}}^{j}+1\right] \delta\left(\Delta \varepsilon^{+}\right)\right]
\end{aligned}
$$

with $\Delta \varepsilon^{ \pm}=\varepsilon_{\mathbf{k}^{\prime}}^{\lambda^{\prime}}-\varepsilon_{\mathbf{k}}^{\lambda} \pm \hbar \omega_{\mp \mathbf{k}^{\prime} \pm \mathbf{k}}^{j}$, where $\mathbf{q} \rightarrow \mathbf{k}^{\prime}-\mathbf{k}$ in the first and $\mathbf{q} \rightarrow \mathbf{k}^{\prime}-\mathbf{k}$ in the second term of the Eqs. F.2 and F.3 was used. The carrier occupation can be written as

$$
\rho_{\mathbf{k}}^{\lambda}(t)=\rho_{\mathbf{k}}^{\lambda, 0}(t)+\delta \rho_{\mathbf{k}}^{\lambda}(t)
$$

where $\rho_{\mathbf{k}}^{\lambda, 0}(t)$ represents the carriers excited by the pump pulse and $\delta \rho_{\mathbf{k}}^{\lambda}(t)$ is a small perturbation induced by the $\mathrm{THz}$ probe pulse. Note, both contributions depend explicitly on time. Assuming thermal equilibrium for the carrier system, all purely pump-induced terms cancels via:

$$
\left.\frac{d}{d t} \rho_{\mathbf{k}}^{\lambda, 0}\right|_{H_{c, p}}=\Gamma_{\lambda, \mathbf{k}}^{\mathrm{in}, c p, 0}\left[1-\rho_{\mathbf{k}}^{0, \lambda}\right]-\Gamma_{\lambda, \mathbf{k}}^{\mathrm{out}, c p, 0} \rho_{\mathbf{k}}^{\lambda, 0} .
$$


Neglecting terms in second order of the perturbation $\mathcal{O}\left(\delta^{2}\right)$, Eqs. (F.1. F.3) yield

$$
\left.\frac{d}{d t} \delta \rho_{\mathbf{k}}^{\lambda}\right|_{H_{c, p}}=\sum_{\lambda^{\prime} \mathbf{k}^{\prime}}\left[\gamma_{\mathbf{k}, \mathbf{k}^{\prime}, \lambda, \lambda^{\prime}}^{0, \text { in }}+\gamma_{\mathbf{k}, \mathbf{k}^{\prime}, \lambda, \lambda^{\prime}}^{\text {out }, 0}\right] \delta \rho_{\mathbf{k}^{\prime}}^{\lambda^{\prime}}-\left[\Gamma_{\lambda, \mathbf{k}}^{\text {in }, c p, 0}+\Gamma_{\lambda, \mathbf{k}}^{\text {out }, c p, 0}\right] \delta \rho_{\mathbf{k}}^{\lambda},
$$

with

$$
\begin{aligned}
\gamma_{\mathbf{k}, \mathbf{k}^{\prime}, \lambda, \lambda^{\prime}}^{\text {in, }}= & \frac{2 \pi}{\hbar} \sum_{j} \times \\
& {\left[\left|g_{\mathbf{k}^{\prime}, \mathbf{k}^{\prime}-\mathbf{k}}^{\lambda^{\prime} \lambda j}\right|^{2}\left(1-\rho_{\mathbf{k}}^{\lambda, 0}\right)\left[n_{\mathbf{k}^{\prime}-\mathbf{k}}^{j}+1\right] \delta\left(\Delta \varepsilon^{-}\right)+\left|g_{\mathbf{k}, \mathbf{k}-\mathbf{k}^{\prime}}^{\lambda^{\prime} \lambda j}\right|^{2}\left(1-\rho_{\mathbf{k}}^{\lambda, 0}\right) n_{\mathbf{k}^{\prime}-\mathbf{k}}^{j} \delta\left(\Delta \varepsilon^{+}\right)\right] }
\end{aligned}
$$

and

$$
\begin{aligned}
\gamma_{\mathbf{k}, \mathbf{k}^{\prime}, \lambda, \lambda^{\prime}}^{\text {out }, 0}= & \frac{2 \pi}{\hbar} \sum_{j} \times \\
& {\left[\left|g_{\mathbf{k}^{\prime}, \mathbf{k}^{\prime}-\mathbf{k}}^{\lambda^{\prime} \lambda j}\right|^{2} \rho_{\mathbf{k}}^{\lambda, 0} n_{\mathbf{k}^{\prime}-\mathbf{k}}^{j} \delta\left(\Delta \varepsilon^{-}\right)+\left|g_{\mathbf{k}, \mathbf{k}-\mathbf{k}^{\prime}}^{\lambda^{\prime} \lambda j}\right|^{2} \rho_{\mathbf{k}}^{\lambda, 0}\left[n_{\mathbf{k}^{\prime}-\mathbf{k}}^{j}+1\right] \delta\left(\Delta \varepsilon^{+}\right)\right] . }
\end{aligned}
$$

With the compound index $i=(\mathbf{k}, \lambda)$ Eqs. F.6 F.9) can be rewritten in a compact way:

$$
\delta \dot{\rho}_{i}=A_{i j} \delta \rho_{i}
$$

with the diagonal elements

$$
A_{i i}=\Gamma_{i}^{\mathrm{in}, c p, 0}+\Gamma_{i}^{\mathrm{out}, c p, 0}
$$

and the off-diagonal elements

$$
A_{i j}=\gamma_{i, j}^{\text {in }, 0}+\gamma_{i, j}^{\text {out }, 0}
$$

\section{F.2. Electron-Electron Scattering}

The Coulomb-induced scattering occupation reads

$$
\left.\frac{d}{d t} \rho_{l}\right|_{H_{c, p}}=\Gamma_{l}^{\mathrm{in}, c c}\left[1-\rho_{l}\right]-\Gamma_{l}^{\mathrm{out}, c c} \rho_{l},
$$

where the in- and out-scattering rates are given by

$$
\begin{aligned}
\Gamma_{l}^{\mathrm{in}, c c} & =\frac{2 \pi}{\hbar} \sum_{a b c} V_{b c}^{l a} \tilde{V}_{l a}^{b c}\left[1-\rho_{a}\right] \rho_{b} \rho_{c} \delta(\Delta \varepsilon), \\
\Gamma_{l}^{\mathrm{out}, c c} & =\frac{2 \pi}{\hbar} \sum_{a b c} V_{b c}^{l a} \tilde{V}_{l a}^{b c} \rho_{a}\left[1-\rho_{b}\right]\left[1-\rho_{c}\right] \delta(\Delta \varepsilon),
\end{aligned}
$$


with $\Delta \varepsilon=\varepsilon_{l}+\varepsilon_{a}-\varepsilon_{b}-\varepsilon_{c}$. The exchange term is neglected in the following, so that $V_{b c}^{l a} \tilde{V}_{l a}^{b c} \approx\left|V_{b c}^{l a}\right|^{2}$. Using the same ansatz and approximations as in section F.1. the occupation in the scattering kernel reads

$$
\begin{gathered}
{\left[1-\rho_{a}\right] \rho_{b} \rho_{c}\left[1-\rho_{1}\right]-\rho_{a}\left[1-\rho_{b}\right]\left[1-\rho_{c}\right] \rho_{l} \approx} \\
-\left[\left[1-\rho_{a}^{0}\right] \rho_{b}^{0} \rho_{c}^{0}+\rho_{a}^{0}\left[1-\rho_{b}^{0}\right]\left[1-\rho_{c}^{0}\right]\right] \delta \rho_{l} \\
-\left[\left[1-\rho_{l}^{0}\right] \rho_{b}^{0} \rho_{c}^{0}+\rho_{l}^{0}\left[1-\rho_{b}^{0}\right]\left[1-\rho_{c}^{0}\right]\right] \delta \rho_{a} \\
\quad+\left[\left[1-\rho_{c}^{0}\right] \rho_{l}^{0} \rho_{a}^{0}+\rho_{c}^{0}\left[1-\rho_{a}^{0}\right]\left[1-\rho_{l}^{0}\right]\right] \delta \rho_{b} \\
+\left[\left[1-\rho_{b}^{0}\right] \rho_{l}^{0} \rho_{a}^{0}+\rho_{b}^{0}\left[1-\rho_{a}^{0}\right]\left[1-\rho_{l}^{0}\right]\right] \delta \rho_{c},
\end{gathered}
$$

which results for the dynamics of the perturbation in

$$
\left.\frac{d}{d t} \delta \rho_{l}\right|_{H_{c, c}}=-\Gamma_{l}^{0} \delta \rho_{l}-\sum_{a} \gamma_{a l}^{0} \delta \rho_{a}+\sum_{b} \gamma_{b l}^{0} \delta \rho_{b}+\sum_{a} \gamma_{c l}^{0} \delta \rho_{\mathbf{c}}
$$

with

$$
\begin{aligned}
& \Gamma_{l}^{0}=\Gamma_{l}^{\text {in }, c c, 0}+\Gamma_{l}^{\text {out }, c c, 0} \\
& \gamma_{a l}^{0}=\frac{2 \pi}{\hbar} \sum_{b c}\left|V_{b c}^{l a}\right|^{2}\left[\gamma_{l b c}^{\text {in }}+\gamma_{l b c}^{\text {out }}\right] \delta(\Delta \varepsilon), \\
& \gamma_{b l}^{0}=\frac{2 \pi}{\hbar} \sum_{c a}\left|V_{b c}^{l a}\right|^{2}\left[\gamma_{c l a}^{\text {in }}+\gamma_{c l a}^{\text {out }}\right] \delta(\Delta \varepsilon), \\
& \gamma_{c l}^{0}=\frac{2 \pi}{\hbar} \sum_{a b}\left|V_{b c}^{l a}\right|^{2}\left[\gamma_{b a l}^{\text {in }}+\gamma_{b a l}^{\text {out }}\right] \delta(\Delta \varepsilon),
\end{aligned}
$$

and

$$
\begin{aligned}
\gamma_{123}^{\text {in }} & =\left[1-\rho_{1}^{0}\right] \rho_{2}^{0} \rho_{3}^{0}, \\
\gamma_{123}^{\text {out }} & =\rho_{1}^{0}\left[1-\rho_{2}^{0}\right]\left[1-\rho_{3}^{0}\right] .
\end{aligned}
$$

\section{F.3. Drude-like intraband current}

For constant scattering rates $\Gamma_{\mathbf{k}}^{\lambda, 0}=\Gamma$ and with the optical intraband matrix element $\mathbf{M}_{\mathbf{k}}^{\lambda \lambda} \approx i \sigma_{\lambda} M \mathbf{e}_{\mathbf{k}}\left(\sigma_{c}=1\right.$ and $\left.\sigma_{v}=-1\right)$ the intraband current can be evaluated analytically using Eqs. 5.6 and 5.7 .

$$
j(\omega)=\frac{e_{0}^{2} M A_{0} k_{B}}{m_{0} \pi c^{2}}\left[i \frac{\Gamma}{\Gamma^{2}+\omega^{2}}-\frac{\omega}{\Gamma^{2}+\omega^{2}}\right] T\left[\ln \left(1+e^{\mu / k_{b} T}\right)+\ln \left(1+e^{-\mu / k_{b} T}\right)\right],
$$

which corresponds to the Drude model. 


\section{G. Carrier and energy density}

In this appendix the carrier and energy density of graphene is evaluated, which is needed to determine the (hot) carrier multiplication. Assuming a Fermi distribution for the carrier occupation $\rho_{\mathbf{k}}^{\lambda}$, the densities read in the case of $\varepsilon_{F} \neq 0$

$$
\begin{aligned}
& n_{\mathrm{CM}}\left(T, \varepsilon_{F}\right)=-\frac{2 k_{B}^{2}}{\pi v_{F}^{2}} T^{2} \sum_{\sigma= \pm} \operatorname{Li}_{2}\left(-e^{\frac{\sigma \varepsilon_{F}}{T k_{B}}}\right), \\
& \mathcal{E}_{\mathrm{CM}}\left(T, \varepsilon_{F}\right)=-\frac{4 k_{B}^{3}}{\pi v_{F}^{2}} T^{3} \sum_{\sigma= \pm} \operatorname{Li}_{3}\left(-e^{\frac{\sigma \varepsilon_{F}}{T k_{B}}}\right),
\end{aligned}
$$

where $\operatorname{Li}_{n}(z)=\sum_{k=1}^{\infty} \frac{z^{k}}{k^{n}}$ denotes the polylogarithm. In the case of hot carrier multiplication, the carrier and energy density is given by

$$
\begin{aligned}
n_{\mathrm{hCM}}\left(T, \varepsilon_{F}\right) & =\frac{\sigma_{s} \sigma_{v}}{L^{2}}\left(\sum_{\mathbf{k}} \rho_{\mathbf{k}}^{h}+\sum_{\mathbf{k}}^{k<k_{F}} \rho_{\mathbf{k}}^{h, c}+\sum_{\mathbf{k}}^{k>k_{F}} \rho_{\mathbf{k}}^{e, c}\right), \\
\mathcal{E}_{\mathrm{CM}}\left(T, \varepsilon_{F}\right) & =\frac{\sigma_{s} \sigma_{v}}{L^{2}}\left(\sum_{\mathbf{k}} \varepsilon_{\mathbf{k}}^{h} \rho_{\mathbf{k}}^{h}+\sum_{\mathbf{k}}^{k<k_{F}} \varepsilon_{\mathbf{k}}^{h, c} \rho_{\mathbf{k}}^{h, c}+\sum_{\mathbf{k}}^{k>k_{F}} \varepsilon_{\mathbf{k}}^{e, c} \rho_{\mathbf{k}}^{e, c}\right)
\end{aligned}
$$

with $\varepsilon_{\mathbf{k}}^{h, c}=\hbar v_{F}\left(k_{F}-k\right)$ for $k<k_{F}, \varepsilon_{\mathbf{k}}^{e, c}=\hbar v_{F}\left(k-k_{F}\right)$ for $k>k_{F}$, and in the bottom cone with $\varepsilon_{\mathbf{k}}^{h, v} \equiv \varepsilon_{\mathbf{k}}^{h}=\hbar v_{F}\left(k+k_{F}\right)$. Inserting the Fermi distribution in Eqs. G.3 and G.4 the densities yield

$$
\begin{aligned}
n_{\mathrm{hCM}}\left(T, \varepsilon_{F}\right)=\frac{1}{6 \pi v_{F}^{2}}[ & -12 k_{B}^{2} T^{2}\left[-\operatorname{Li}_{2}\left(-e^{\frac{\varepsilon_{F}}{k_{B} T}}\right)+\operatorname{Li}_{2}\left(-e^{\frac{2 \varepsilon_{F}}{k_{B} T}}\right)+\operatorname{Li}_{2}\left(-e^{\frac{\varepsilon_{F}}{k_{B} T}}\right)\right]+\pi^{2} k_{B}^{2} T^{2} \\
& \left.-12 k_{B} \varepsilon_{F} T \log \left(e^{\frac{2 \varepsilon_{F}}{k_{B} T}}+1\right)+6 \varepsilon_{F}\left(k_{B} T \log (4)+\varepsilon_{F}\right)\right] \\
\mathcal{E}_{\mathrm{hCM}}\left(T, \varepsilon_{F}\right)=\frac{1}{6 \pi v_{F}^{2}}[ & 6 k_{B}^{2} T^{2}\left[k_{B} T\left(3 \zeta(3)-4\left(\operatorname{Li}_{3}\left(-e^{\frac{\varepsilon_{F}}{k_{B} T}}\right)-\mathrm{Li}_{3}\left(-e^{\frac{2 \varepsilon_{F}}{k_{B} T}}\right)+\operatorname{Li}_{3}\left(-e^{\frac{\varepsilon_{F}}{k_{B} T}}\right)\right)\right)\right. \\
& \left.-2 \varepsilon_{F}\left[\operatorname{Li}_{2}\left(-e^{\frac{\varepsilon_{F}}{k_{B} T}}\right)+\operatorname{Li}_{2}\left(-e^{\frac{2 \varepsilon_{F}}{k_{B} T}}\right)+\operatorname{Li}_{2}\left(-e^{\frac{\varepsilon_{F}}{k_{B} T}}\right)\right]+\pi^{2} k_{B}^{2} \varepsilon_{F} T^{2}-2 \varepsilon_{F}^{3}\right] .
\end{aligned}
$$

For undoped graphene, both definitions of the carrier and energy density are equivalent for symmetry reasons. For a vanishing Fermi level, the polylogarithm 
can be analytically determined resulting in $\operatorname{Li}_{2}(-1)=-\pi^{2} / 12$ and $\operatorname{Li}_{3}(-1)=$ $-3 \zeta(3) / 4$. Therewith, the densities yield

$$
\begin{aligned}
n(T) & =\frac{\pi k_{B}^{2}}{3 v_{F}^{2}} T^{2}, \\
\mathcal{E}(T) & =\frac{6 \zeta(3) k_{B}^{3}}{\pi v_{F}^{2}} T^{3},
\end{aligned}
$$

where $\zeta($.$) denotes the Zeta function.$ 


\section{List of Figures}

1.1. Graphitic materials in various dimensionalities. . . . . . . . . . 2

2.1. Structure of the graphene lattice. . . . . . . . . . . . . . . 6

2.2. Structure of bilayer graphene . . . . . . . . . . . . 7

2.3. Bandstructure of the graphene . . . . . . . . . . . . . . . 9 9

2.4. Band structure of the bilayer graphene . . . . . . . . . . . . . 12

2.5. Optical matrix element of graphene . . . . . . . . . . . . . . . . . . . . 13

2.6. Optical matrix element of bilayer graphene . . . . . . . . . . . . . . . . 15

2.7. Coulomb-induced scattering channels . . . . . . . . . . . . . . 17

2.8. Dielectrical function as a function of the momentum transfer . . . . 18

2.9. Phonon dispersion of graphene. . . . . . . . . . . . . 20

2.10. Microscopic quantities . . . . . . . . . . . . . . . . 21

2.11. Scheme of the differential transmission spectroscopy . . . . . . . . . 27

3.1. Temporal evolution of the polarization at the $M$ point. . . . . . . . 31

3.2. $\quad$ Exitonic absorption spectrum of graphene . . . . . . . . . . . . . . 32

3.3. $\quad$ Excitonic absorption spectrum of bilayer graphene . . . . . . . . . . 34

3.4. Free-particle, renormalized, and excitonic absorption spectrum of bilayer graphene . . . . . . . . . . . . . . . . 35

4.1. Illustration of the phonon-assisted intraband absorption processes . 37

4.2. Free-particle absorption spectrum of graphene including only the contribution of the intraband absorption . . . . . . . . . . 42

4.3. Transient differential transmission spectrum with the impact of phononassisted intraband processes . . . . . . . . . . . . . 43

4.4. Transient differential transmission spectrum in comparison with the

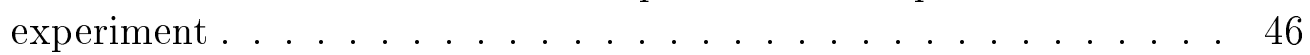

5.1. Hot-carrier relaxation and cooling dynamics of graphene with various doping densities . . . . . . . . . . . . . . . . 49

5.2. Optical pump and THz probe DTS of highly doped graphene . . . . 54

5.3. $\mathrm{THz}$ carrier relaxation times in highly doped graphene $\ldots . . .55$

5.4. Optical pump and THz probe DTS of graphene with low doping

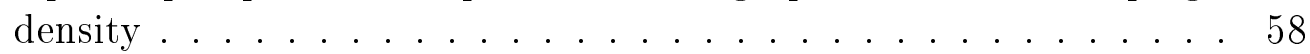


5.5. $\mathrm{THz}$ carrier relaxation times in graphene with low doping density. . 59

5.6. Microscopic theory calculation of carrier dynamics and carrier scat-

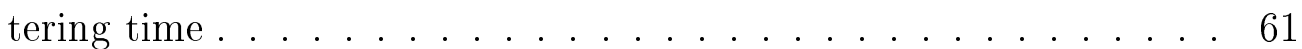

5.7. Microscopic theory calculation of carrier dynamics and carrier scattering time...................... 64

6.1. Occupation probability of doped graphene. . . . . . . . . . . . 67

6.2 . Doping dependence of the microscopic polarization. . . . . . . . . . 69

6.3. Fully momentum resolved carrier occupation $\rho_{k, \phi}$ as a function of the momentum $\mathrm{k}$ and the orientation angle $\phi$ with respect to the

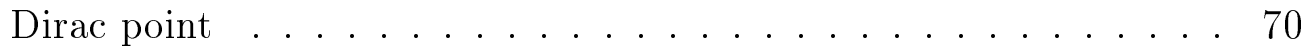

6.4. Carrier occupation $\rho_{k, \phi_{k}}$ as a function of the momentum $\mathrm{k}$ and the orientation angle $\phi$ around the Dirac point for n-doped graphene with an excitation near the Fermi level . . . . . . . . . . . . 71

6.5. Schematic illustration of the impact excitation in the linear regime of the electronic band structure for n-doped graphene . . . . . . . 72

6.6. Temporal evolution of the doping-dependent carrier multiplication . 73

6.7. Temporal evolution of Auger rates . . . . . . . . . . . . 74

6.8. Temporal evolution of the doping-dependent hot carrier multiplication 77

6.9. Analytically obtained carrier multiplication as a function of doping. 79

6.10. Doping-dependent carrier multiplication for different pump fluences

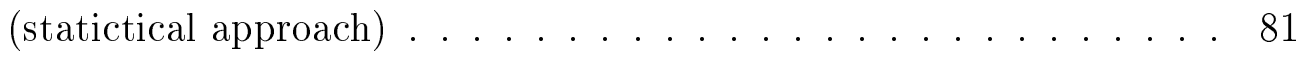

6.11. Doping-dependent hot carrier multiplication for different pump fluences $($ statictical approach) $\ldots \ldots \ldots \ldots . \ldots . \ldots . \ldots 2$ 


\section{Bibliography}

[1] K. S. Novoselov, A. K. Geim, S. V. Morozov, D. Jiang, Y. Zhang, S. V. Dubonos, I. V. Grigorieva, and A. A. Firsov. Electric field effect in atomically thin carbon films. Science, 306(5696):666-669, October 2004. PMID: 15499015 .

[2] R. Peierls. Quelques propriétés typiques des corps solides. Annales de l'institut Henri Poincaré, 5(3):177-222, 1935.

[3] LD Landau. Zur theorie der phasenumwandlungen ii. Phys. Z. Sowjetunion, 11:26-35, 1937.

[4] Yuanbo Zhang, Yan-Wen Tan, Horst L. Stormer, and Philip Kim. Experimental observation of the quantum hall effect and berry's phase in graphene. Nature, 438(7065):201-204, November 2005.

[5] K. S. Novoselov, A. K. Geim, S. V. Morozov, D. Jiang, M. I. Katsnelson, I. V. Grigorieva, S. V. Dubonos, and A. A. Firsov. Two-dimensional gas of massless dirac fermions in graphene. Nature, 438(7065):197-200, November 2005 .

[6] A. K. Geim and K. S. Novoselov. The rise of graphene. Nature Materials, 6(3):183-191, 2007.

[7] K. S. Novoselov, E. McCann, S. V. Morozov, V. I. Fal/'ko, M. I. Katsnelson, U. Zeitler, D. Jiang, F. Schedin, and A. K. Geim. Unconventional quantum Hall effect and Berry/'s phase of 2[pi] in bilayer graphene. Nat Phys, $2(3): 177-180,2006$.

[8] Li Yang, Jack Deslippe, Cheol-Hwan Park, Marvin L. Cohen, and Steven G. Louie. Excitonic effects on the optical response of graphene and bilayer graphene. Physical Review Letters, 103(18):186802, October 2009.

[9] Edward McCann and Mikito Koshino. The electronic properties of bilayer graphene. Reports on Progress in Physics, 76(5):056503, 2013. 
[10] Kin Fai Mak, Jie Shan, and Tony F. Heinz. Electronic structure of few-layer graphene: Experimental demonstration of strong dependence on stacking sequence. Phys. Rev. Lett., 104:176404, Apr 2010.

[11] Taisuke Ohta, Aaron Bostwick, Thomas Seyller, Karsten Horn, and Eli Rotenberg. Controlling the electronic structure of bilayer graphene. Science, 313(5789):951-954, 2006.

[12] Kin Fai Mak, Chun Hung Lui, Jie Shan, and Tony F. Heinz. Observation of an electric-field-induced band gap in bilayer graphene by infrared spectroscopy. Phys. Rev. Lett., 102:256405, Jun 2009.

[13] F. Guinea, A. H. Castro Neto, and N. M. R. Peres. Electronic states and landau levels in graphene stacks. Phys. Rev. B, 73:245426, Jun 2006.

[14] Eduardo V. Castro, K. S. Novoselov, S. V. Morozov, N. M. R. Peres, J. M. B. Lopes dos Santos, Johan Nilsson, F. Guinea, A. K. Geim, and A. H. Castro Neto. Biased bilayer graphene: Semiconductor with a gap tunable by the electric field effect. Phys. Rev. Lett., 99:216802, Nov 2007.

[15] E. J. Nicol and J. P. Carbotte. Optical conductivity of bilayer graphene with and without an asymmetry gap. Phys. Rev. B, 77:155409, Apr 2008.

[16] B. N. Szafranek, D. Schall, M. Otto, D. Neumaier, and H. Kurz. High on/off ratios in bilayer graphene field effect transistors realized by surface dopants. Nano Letters, 11(7):2640-2643, 2011. PMID: 21688768.

[17] Ermin Malic, Torben Winzer, Evgeny Bobkin, and Andreas Knorr. Microscopic theory of absorption and ultrafast many-particle kinetics in graphene. Physical Review B, 84(20):205406, November 2011.

[18] Jahan M. Dawlaty, Shriram Shivaraman, Mvs Chandrashekhar, Farhan Rana, and Michael G. Spencer. Measurement of ultrafast carrier dynamics in epitaxial graphene. Applied Physics Letters, 92(4):042116-042116-3, January 2008.

[19] P. Plochocka, P. Kossacki, A. Golnik, T. Kazimierczuk, C. Berger, W. A. de Heer, and M. Potemski. Slowing hot-carrier relaxation in graphene using a magnetic field. Physical Review B, 80(24):245415, December 2009.

[20] Haining Wang, Jared H. Strait, Paul A. George, Shriram Shivaraman, Virgil B. Shields, Mvs Chandrashekhar, Jeonghyun Hwang, Farhan Rana, Michael G. Spencer, Carlos S. Ruiz-Vargas, and Jiwoong Park. Ultrafast relaxation dynamics of hot optical phonons in graphene. Applied Physics Letters, 96(8):081917-081917-3, February 2010. 
[21] Xingquan Zou, Da Zhan, Xiaofeng Fan, Dongwook Lee, Saritha K. Nair, Li Sun, Zhenhua Ni, Zhiqiang Luo, Lei Liu, Ting Yu, Zexiang Shen, and Elbert E. M. Chia. Ultrafast carrier dynamics in pristine and $\mathrm{FeCl3-}$ intercalated bilayer graphene. Applied Physics Letters, 97(14):141910141910-3, October 2010.

[22] M. Breusing, S. Kuehn, T. Winzer, E. Malić, F. Milde, N. Severin, J. P. Rabe, C. Ropers, A. Knorr, and T. Elsaesser. Ultrafast nonequilibrium carrier dynamics in a single graphene layer. Physical Review B, 83(15):153410, April 2011.

[23] S. Winnerl, M. Orlita, P. Plochocka, P. Kossacki, M. Potemski, T. Winzer, E. Malic, A. Knorr, M. Sprinkle, C. Berger, W. A. de Heer, H. Schneider, and M. Helm. Carrier relaxation in epitaxial graphene photoexcited near the dirac point. Physical Review Letters, 107(23):237401, November 2011.

[24] Leandro M Malard, Kin Fai Mak, A H Castro Neto, N M R Peres, and Tony F Heinz. Observation of intra- and inter-band transitions in the transient optical response of graphene. New Journal of Physics, 15(1):015009, 2013.

[25] Petr A. Obraztsov, Maxim G. Rybin, Anastasia V. Tyurnina, Sergey V. Garnov, Elena D. Obraztsova, Alexander N. Obraztsov, and Yuri P. Svirko. Broadband light-induced absorbance change in multilayer graphene. Nano Letters, 11(4):1540-1545, April 2011.

[26] S. Winnerl, F. Göttfert, M. Mittendorff, H. Schneider, M. Helm, T. Winzer, E. Malic, A. Knorr, M. Orlita, M. Potemski, M. Sprinkle, C. Berger, and W. A. de Heer. Time-resolved spectroscopy on epitaxial graphene in the infrared spectral range: relaxation dynamics and saturation behavior. Journal of Physics: Condensed Matter, 25(5):054202, February 2013.

[27] Torben Winzer, Andreas Knorr, Martin Mittendorff, Stephan Winnerl, Miao-Bin Lien, Dong Sun, Theodore B. Norris, Manfred Helm, and Ermin Malic. Absorption saturation in optically excited graphene. Applied Physics Letters, 101(22):221115-221115-4, November 2012.

[28] Ermin Malic, Torben Winzer, and Andreas Knorr. Efficient orientational carrier relaxation in optically excited graphene. Applied Physics Letters, 101(21):213110-213110-3, November 2012.

[29] Martin Mittendorff, Torben Winzer, Ermin Malic, Andreas Knorr, Claire Berger, Walter A. de Heer, Harald Schneider, Manfred Helm, and Stephan 
Winnerl. Anisotropy of excitation and relaxation of photogenerated charge carriers in graphene. Nano Letters, 14(3):1504-1507, 2014. PMID: 24559191.

[30] Torben Winzer, Andreas Knorr, and Ermin Malic. Carrier multiplication in graphene. Nano Letters, 10(12):4839-4843, December 2010.

[31] Torben Winzer, Ermin Malić, and Andreas Knorr. Microscopic mechanism for transient population inversion and optical gain in graphene. Physical Review B, 87(16):165413, April 2013.

[32] D. Brida, A. Tomadin, C. Manzoni, Y. J. Kim, A. Lombardo, S. Milana, R. R. Nair, K. S. Novoselov, A. C. Ferrari, G. Cerullo, and M. Polini. Ultrafast collinear scattering and carrier multiplication in graphene. Nature Communications, 4, June 2013.

[33] Justin C. W. Song, Klaas J. Tielrooij, Frank H. L. Koppens, and Leonid S. Levitov. Photoexcited carrier dynamics and impact-excitation cascade in graphene. Physical Review B, 87(15):155429, April 2013.

[34] T. Li, L. Luo, M. Hupalo, J. Zhang, M. C. Tringides, J. Schmalian, and J. Wang. Femtosecond population inversion and stimulated emission of dense dirac fermions in graphene. Physical Review Letters, 108(16):167401, April 2012 .

[35] T. Ploetzing, T. Winzer, E. Malic, D. Neumaier, A. Knorr, and H. Kurz. Experimental verification of carrier multiplication in graphene. Nano Letters, 14(9):5371-5375, 2014. PMID: 25144320.

[36] Fausto Rossi and Tilmann Kuhn. Theory of ultrafast phenomena in photoexcited semiconductors. Reviews of Modern Physics, 74(3):895-950, August 2002 .

[37] M. Kira, F. Jahnke, W. Hoyer, and S.W. Koch. Quantum theory of spontaneous emission and coherent effects in semiconductor microstructures. Progress in Quantum Electronics, 23(6):189-279, November 1999.

[38] Marlan O. Scully and M. Suhail Zubairy. Quantum Optics. Cambridge University Press, 1 edition, September 1997.

[39] E. H. Hwang and S. Das Sarma. Acoustic phonon scattering limited carrier mobility in two-dimensional extrinsic graphene. Physical Review B, 77(11):115449, March 2008. 
[40] S. Piscanec, M. Lazzeri, Francesco Mauri, A. C. Ferrari, and J. Robertson. Kohn anomalies and electron-phonon interactions in graphite. Physical Review Letters, 93(18):185503, October 2004.

[41] Ermin Malic and Andreas Knorr. Graphene and Carbon Nanotubes: Ultrafast Optics and Relaxation Dynamics. Wiley-VCH, 1 edition, May 2013.

[42] A. Grüneis, C. Attaccalite, L. Wirtz, H. Shiozawa, R. Saito, T. Pichler, and A. Rubio. Tight-binding description of the quasiparticle dispersion of graphite and few-layer graphene. Phys. Rev. B, 78:205425, Nov 2008.

[43] S. Reich, J. Maultzsch, C. Thomsen, and P. Ordejón. Tight-binding description of graphene. Physical Review B, 66(3):035412, July 2002.

[44] Edward McCann and Mikito Koshino. The electronic properties of bilayer graphene. Reports on Progress in Physics, 76(5):056503, 2013.

[45] A. Grüneis, C. Attaccalite, L. Wirtz, H. Shiozawa, R. Saito, T. Pichler, and A. Rubio. Tight-binding description of the quasiparticle dispersion of graphite and few-layer graphene. Phys. Rev. B, 78:205425, Nov 2008.

[46] Faris Kadi and Ermin Malic. Optical properties of bernal-stacked bilayer graphene: A theoretical study. Phys. Rev. B, 89:045419, Jan 2014.

[47] Edward McCann. Asymmetry gap in the electronic band structure of bilayer graphene. Phys. Rev. B, 74:161403, Oct 2006.

[48] Ermin Malić, Matthias Hirtschulz, Frank Milde, Andreas Knorr, and Stephanie Reich. Analytical approach to optical absorption in carbon nanotubes. Physical Review B, 74(19):195431, November 2006.

[49] A. Grüneis, R. Saito, Ge. G. Samsonidze, T. Kimura, M. A. Pimenta, A. Jorio, A. G. Souza Filho, G. Dresselhaus, and M. S. Dresselhaus. Inhomogeneous optical absorption around the $\mathrm{k}$ point in graphite and carbon nanotubes. Physical Review B, 67(16):165402, April 2003.

[50] T. Winzer. Ultrafast Carrier Relaxation Dynamics in Graphene. PhD thesis, Technische Universität Berlin, July 2013.

[51] Hartmut Haug and Stephan W. Koch. Quantum Theory of the Optical and Electronic Properties of Semiconductors. World Scientific, 2004.

[52] G.F. Guiliani and G Vignale. Quantum Theory of the Electron Liquid. Cambridge University Press, 2005. 
[53] J. Maultzsch, S. Reich, C. Thomsen, H. Requardt, and P. Ordejón. Phonon dispersion in graphite. Physical Review Letters, 92(7):075501, February 2004.

[54] G. D. Sanders, A. R. T. Nugraha, K. Sato, J.-H. Kim, J. Kono, R. Saito, and C. J. Stanton. Theory of coherent phonons in carbon nanotubes and graphene nanoribbons. Journal of Physics: Condensed Matter, 25(14):144201, April 2013.

[55] Wang-Kong Tse and S. Das Sarma. Energy relaxation of hot dirac fermions in graphene. Physical Review B, 79(23):235406, June 2009.

[56] Faris Kadi, Torben Winzer, Ermin Malic, Andreas Knorr, F. Göttfert, M. Mittendorff, S. Winnerl, and M. Helm. Microscopic description of intraband absorption in graphene: The occurrence of transient negative differential transmission. Phys. Rev. Lett., 113:035502, Jul 2014.

[57] Ermin Malić, Matthias Hirtschulz, Frank Milde, Yang Wu, Janina Maultzsch, Tony F. Heinz, Andreas Knorr, and Stephanie Reich. Theory of rayleigh scattering from metallic carbon nanotubes. Physical Review B, 77(4):045432, January 2008.

[58] Ermin Malic, Janina Maultzsch, Stephanie Reich, and Andreas Knorr. Excitonic absorption spectra of metallic single-walled carbon nanotubes. Physical Review B, 82(3):035433, July 2010.

[59] Ermin Malic, Janina Maultzsch, Stephanie Reich, and Andreas Knorr. Excitonic rayleigh scattering spectra of metallic single-walled carbon nanotubes. Physical Review B, 82(11):115439, September 2010.

[60] S. Butscher, F. Milde, M. Hirtschulz, E. Malić, and A. Knorr. Hot electron relaxation and phonon dynamics in graphene. Applied Physics Letters, 91(20):203103-203103-3, November 2007.

[61] Kwangu Kang, Daner Abdula, David G. Cahill, and Moonsub Shim. Lifetimes of optical phonons in graphene and graphite by time-resolved incoherent anti-stokes raman scattering. Physical Review B, 81(16):165405, April 2010 .

[62] Shaul Mukamel. Principles of Nonlinear Optical Spectroscopy. Oxford University Press, USA, April 1999.

[63] Kin Fai Mak, Matthew Y. Sfeir, James A. Misewich, and Tony F. Heinz. The evolution of electronic structure in few-layer graphene revealed by optical spectroscopy. Proceedings of the National Academy of Sciences, 107(34):14999-15004, 2010. 
[64] Matthias Hirtschulz, Frank Milde, Ermin Malić, Stefan Butscher, Christian Thomsen, Stephanie Reich, and Andreas Knorr. Carbon nanotube bloch equations: A many-body approach to nonlinear and ultrafast optical properties. Physical Review B, 77(3):035403, January 2008.

[65] E. Malic, C. Weber, M. Richter, V. Atalla, T. Klamroth, P. Saalfrank, S. Reich, and A. Knorr. Microscopic model of the optical absorption of carbon nanotubes functionalized with molecular spiropyran photoswitches. Physical Review Letters, 106(9):097401, March 2011.

[66] Kin Fai Mak, Jie Shan, and Tony F. Heinz. Seeing many-body effects in single- and few-layer graphene: Observation of two-dimensional saddle-point excitons. Physical Review Letters, 106(4):046401, January 2011.

[67] Dong-Hun Chae, Tobias Utikal, Siegfried Weisenburger, Harald Giessen, Klaus v. Klitzing, Markus Lippitz, and Jurgen Smet. Excitonic fano resonance in free-standing graphene. Nano Letters, 11(3):1379-1382, 2011. PMID: 21322607.

[68] Li Yang. First-principles study of the optical absorption spectra of electrically gated bilayer graphene. Phys. Rev. B, 81:155445, Apr 2010.

[69] Li Yang. Excitons in intrinsic and bilayer graphene. Phys. Rev. B, 83:085405, Feb 2011.

[70] C. P. Chang. Analytic model of energy spectrum and absorption spectra of bilayer graphene. Journal of Applied Physics, 111(10):-, 2012.

[71] Yu A. Ill'inskii and L.V. Keldysh. Electromagnetic Response of Material Media. Springer, 2013.

[72] T. Stroucken, A. Knorr, P. Thomas, and S. W. Koch. Coherent dynamics of radiatively coupled quantum-well excitons. Phys. Rev. B, 53:2026-2033, Jan 1996.

[73] T. Stroucken, J. H. Grönqvist, and S. W. Koch. Excitonic resonances as fingerprint of strong coulomb coupling in graphene. Journal of the Optical Society of America B, 29(2):A86-A94, February 2012.

[74] Eike Verdenhalven and Ermin Malic. Microscopic derivation of many-particle screening in graphene. Research work, TU Berlin, 2013.

[75] T. Stroucken, J. H. Grönqvist, and S. W. Koch. Optical response and ground state of graphene. Physical Review B, 84(20):205445, November 2011. 
[76] Feng Wang, Gordana Dukovic, Louis E. Brus, and Tony F. Heinz. The optical resonances in carbon nanotubes arise from excitons. Science, 308(5723):838$841,2005$.

[77] J. Maultzsch, R. Pomraenke, S. Reich, E. Chang, D. Prezzi, A. Ruini, E. Molinari, M. S. Strano, C. Thomsen, and C. Lienau. Exciton binding energies in carbon nanotubes from two-photon photoluminescence. Phys. Rev. B, 72:241402, Dec 2005.

[78] Feng Wang, David J. Cho, Brian Kessler, Jack Deslippe, P. James Schuck, Steven G. Louie, Alex Zettl, Tony F. Heinz, and Y. Ron Shen. Observation of excitons in one-dimensional metallic single-walled carbon nanotubes. Phys. Rev. Lett., 99:227401, Nov 2007.

[79] Jack Deslippe, Catalin D. Spataru, David Prendergast, and Steven G. Louie. Bound excitons in metallic single-walled carbon nanotubes. Nano Letters, 7(6):1626-1630, 2007. PMID: 17508770.

[80] Dong-Hun Chae, Tobias Utikal, Siegfried Weisenburger, Harald Giessen, Klaus v. Klitzing, Markus Lippitz, and Jurgen Smet. Excitonic fano resonance in free-standing graphene. Nano Letters, 11(3):1379-1382, 2011. PMID: 21322607.

[81] Dong Sun, Zong-Kwei Wu, Charles Divin, Xuebin Li, Claire Berger, Walt A. de Heer, Phillip N. First, and Theodore B. Norris. Ultrafast relaxation of excited dirac fermions in epitaxial graphene using optical differential transmission spectroscopy. Physical Review Letters, 101(15):157402, October 2008.

[82] Jared H. Strait, Haining Wang, Shriram Shivaraman, Virgil Shields, Michael Spencer, and Farhan Rana. Very slow cooling dynamics of photoexcited carriers in graphene observed by optical-pump terahertz-probe spectroscopy. Nano Letters, 11(11):4902-4906, November 2011.

[83] Sunil Kumar, M. Anija, N. Kamaraju, K. S. Vasu, K. S. Subrahmanyam, A. K. Sood, and C. N. R. Rao. Femtosecond carrier dynamics and saturable absorption in graphene suspensions. Applied Physics Letters, 95(19):191911191911-3, November 2009.

[84] Ryan W. Newson, Jesse Dean, Ben Schmidt, and Henry M. van Driel. Ultrafast carrier kinetics in exfoliated graphene and thin graphite films. Optics Express, 17(4):2326-2333, February 2009.

[85] Dong Sun, Charles Divin, Claire Berger, Walt A. de Heer, Phillip N. First, and Theodore B. Norris. Spectroscopic measurement of interlayer screening 
in multilayer epitaxial graphene. Physical Review Letters, 104(13):136802, April 2010.

[86] Jingzhi Shang, Zhiqiang Luo, Chunxiao Cong, Jianyi Lin, Ting Yu, and Gagik G. Gurzadyan. Femtosecond UV-pump/visible-probe measurements of carrier dynamics in stacked graphene films. Applied Physics Letters, 97(16):163103-163103-3, October 2010.

[87] A. T. Roberts, R. Binder, N. H. Kwong, D. Golla, D. Cormode, B. J. Leroy, H. O. Everitt, and A. Sandhu. Optical characterization of electron-phonon interactions at the saddle point in graphene. ArXiv e-prints, October 2013.

[88] J. Schilp, T. Kuhn, and G. Mahler. Electron-phonon quantum kinetics in pulse-excited semiconductors: Memory and renormalization effects. Physical Review B, 50(8):5435-5447, August 1994.

[89] Fausto Rossi, Stefan Haas, and Tilmann Kuhn. Ultrafast relaxation of photoexcited carriers: The role of coherence in the generation process. Physical Review Letters, 72(1):152-155, January 1994.

[90] Mackillo Kira, Walter Hoyer, and Stephan W. Koch. Microscopic theory of the semiconductor terahertz response. physica status solidi (b), 238(3):443450, 2003.

[91] J. E. Sipe and Ed Ghahramani. Nonlinear optical response of semiconductors in the independent-particle approximation. Physical Review B, 48(16):11705-11722, October 1993.

[92] T. Stauber, N. M. R. Peres, and A. K. Geim. Optical conductivity of graphene in the visible region of the spectrum. Phys. Rev. B, 78:085432, Aug 2008.

[93] R. R. Nair, P. Blake, A. N. Grigorenko, K. S. Novoselov, T. J. Booth, T. Stauber, N. M. R. Peres, and A. K. Geim. Fine structure constant defines visual transparency of graphene. Science, 320(5881):1308, 2008.

[94] Kin Fai Mak, Matthew Y. Sfeir, Yang Wu, Chun Hung Lui, James A. Misewich, and Tony F. Heinz. Measurement of the optical conductivity of graphene. Phys. Rev. Lett., 101:196405, Nov 2008.

[95] T. Winzer and E. Malic. The impact of pump fluence on carrier relaxation dynamics in optically excited graphene. Journal of Physics: Condensed Matter, 25(5):054201, February 2013. 
[96] Paul A. George, Jared Strait, Jahan Dawlaty, Shriram Shivaraman, Mvs Chandrashekhar, Farhan Rana, and Michael G. Spencer. Ultrafast opticalpump terahertz-probe spectroscopy of the carrier relaxation and recombination dynamics in epitaxial graphene. Nano Letters, 8(12):4248-4251, December 2008 .

[97] Callum J. Docherty, Cheng-Te Lin, Hannah J. Joyce, Robin J. Nicholas, Laura M. Herz, Lain-Jong Li, and Michael B. Johnston. Extreme sensitivity of graphene photoconductivity to environmental gases. Nat Commun, 3:1228, November 2012.

[98] Giriraj Jnawali, Yi Rao, Hugen Yan, and Tony F. Heinz. Observation of a transient decrease in terahertz conductivity of single-layer graphene induced by ultrafast optical excitation. Nano Letters, 13(2):524-530, 2013. PMID: 23330567.

[99] S.-F. Shi, T.-T. Tang, B. Zeng, L. Ju, Q. Zhou, A. Zettl, and F. Wang. Controlling graphene ultrafast hot carrier response from metal-like to semiconductor-like by electrostatic gating. Nano Letters, 14(3):1578-1582, 2014. PMID: 24564302.

[100] A. J. Frenzel, C. H. Lui, W. Fang, N. L. Nair, P. K. Herring, P. JarilloHerrero, J. Kong, and N. Gedik. Observation of suppressed terahertz absorption in photoexcited graphene. Applied Physics Letters, 102(11):-, 2013.

[101] K. J. Tielrooij, J. C. W. Song, S. A. Jensen, A. Centeno, A. Pesquera, A. Zurutuza Elorza, M. Bonn, L. S. Levitov, and F. H. L. Koppens. Photoexcitation cascade and multiple hot-carrier generation in graphene. Nature Physics, advance online publication, February 2013.

[102] S. A. Jensen, Z. Mics, I. Ivanov, H. S. Varol, D. Turchinovich, F. H. L. Koppens, M. Bonn, and K. J. Tielrooij. Competing ultrafast energy relaxation pathways in photoexcited graphene. Nano Letters, 14(10):5839-5845, 2014. PMID: 25247639.

[103] A. J. Frenzel, C. H. Lui, Y. C. Shin, J. Kong, and N. Gedik. Semiconductingto-metallic photoconductivity crossover and temperature-dependent drude weight in graphene. Phys. Rev. Lett., 113:056602, Jul 2014.

[104] R. Bistritzer and A. H. MacDonald. Electronic cooling in graphene. Phys. Rev. Lett., 102:206410, May 2009. 
[105] Justin C. W. Song, Michael Y. Reizer, and Leonid S. Levitov. Disorderassisted electron-phonon scattering and cooling pathways in graphene. Physical Review Letters, 109(10):106602, September 2012.

[106] M. T. Mihnev, J. R. Tolsma, C. J. Divin, R. Asgari D. Sun, M. Polini, C. Berger, W. A. de Heer, A. H. MacDonald, and T. B. Norris. Electronic cooling via interlayer coulomb coupling in multilayer epitaxial graphene. submitted.

[107] F. Kadi, M. T. Mihnev, C. J. Divin, T. Winzer, S. Lee, C. Liu, Z. Zhong, C. Berger, W. A. de Heer, E. Malic, A. Knorr, and T. B. Norris. Microscopic origins of the terahertz carrier relaxation and cooling dynamics in graphene. submitted.

[108] Isabella Gierz, Jesse C. Petersen, Matteo Mitrano, Cephise Cacho, I. C. Edmond Turcu, Emma Springate, Alexander Stöhr, Axel Köhler, Ulrich Starke, and Andrea Cavalleri. Snapshots of non-equilibrium dirac carrier distributions in graphene. Nature Materials, 12(12):1119-1124, December 2013.

[109] Charles A. Schmuttenmaer. Exploring dynamics in the far-infrared with terahertz spectroscopy. Chemical Reviews, 104(4):1759-1780, 2004. PMID: 15080711.

[110] Jason B. Baxter and Glenn W. Guglietta. Terahertz spectroscopy. Analytical Chemistry, 83(12):4342-4368, 2011. PMID: 21534575.

[111] J. Hass, F. Varchon, J. E. Millán-Otoya, M. Sprinkle, N. Sharma, W. A. de Heer, C. Berger, P. N. First, L. Magaud, and E. H. Conrad. Why multilayer graphene on $4 h-\mathrm{SiC}(000 \overline{1})$ behaves like a single sheet of graphene. Phys. Rev. Lett., 100:125504, Mar 2008.

[112] M. Sprinkle, D. Siegel, Y. Hu, J. Hicks, A. Tejeda, A. Taleb-Ibrahimi, P. Le Fèvre, F. Bertran, S. Vizzini, H. Enriquez, S. Chiang, P. Soukiassian, C. Berger, W. A. de Heer, A. Lanzara, and E. H. Conrad. First direct observation of a nearly ideal graphene band structure. Phys. Rev. Lett., 103:226803, Nov 2009.

[113] Yufeng Hao, M. S. Bharathi, Lei Wang, Yuanyue Liu, Hua Chen, Shu Nie, Xiaohan Wang, Harry Chou, Cheng Tan, Babak Fallahazad, H. Ramanarayan, Carl W. Magnuson, Emanuel Tutuc, Boris I. Yakobson, Kevin F. McCarty, Yong-Wei Zhang, Philip Kim, James Hone, Luigi Colombo, and Rodney S. Ruoff. The role of surface oxygen in the growth of large single-crystal graphene on copper. Science, 342(6159):720-723, 2013. 
[114] Seunghyun Lee, Kyunghoon Lee, and Zhaohui Zhong. Wafer scale homogeneous bilayer graphene films by chemical vapor deposition. Nano Letters, 10(11):4702-4707, 2010. PMID: 20932046.

[115] Claire Berger, Zhimin Song, Xuebin Li, Xiaosong Wu, Nate Brown, Cécile Naud, Didier Mayou, Tianbo Li, Joanna Hass, Alexei N. Marchenkov, Edward H. Conrad, Phillip N. First, and Walt A. de Heer. Electronic confinement and coherence in patterned epitaxial graphene. Science, 312(5777):1191-1196, 2006.

[116] Walt A. de Heer, Claire Berger, Ming Ruan, Mike Sprinkle, Xuebin Li, Yike $\mathrm{Hu}$, Baiqian Zhang, John Hankinson, and Edward Conrad. Large area and structured epitaxial graphene produced by confinement controlled sublimation of silicon carbide. Proceedings of the National Academy of Sciences, 108(41):16900-16905, 2011.

[117] You-Chia Chang, Chang-Hua Liu, Che-Hung Liu, Zhaohui Zhong, and Theodore B. Norris. Extracting the complex optical conductivity of monoand bilayer graphene by ellipsometry. Applied Physics Letters, 104(26):-, 2014 .

[118] Matt W. Graham, Su-Fei Shi, Daniel C. Ralph, Jiwoong Park, and Paul L. McEuen. Photocurrent measurements of supercollision cooling in graphene. 9(2):103-108.

[119] A. C. Betz, S. H. Jhang, E. Pallecchi, R. Ferreira, G. Feve, J-M. Berroir, and B. Placais. Supercollision cooling in undoped graphene. Nat Phys, 9(2):109-112, February 2013.

[120] S. Xu, J. Cao, C. C. Miller, D. A. Mantell, R. J. D. Miller, and Y. Gao. Energy dependence of electron lifetime in graphite observed with femtosecond photoemission spectroscopy. Phys. Rev. Lett., 76:483-486, Jan 1996.

[121] J. González, F. Guinea, and M. A. H. Vozmediano. Unconventional quasiparticle lifetime in graphite. Phys. Rev. Lett., 77:3589-3592, Oct 1996.

[122] Tsuneya Ando. Screening effect and impurity scattering in monolayer graphene. Journal of the Physical Society of Japan, 75(7):074716, 2006.

[123] E. H. Hwang, Rajdeep Sensarma, and S. Das Sarma. Coulomb drag in monolayer and bilayer graphene. Physical Review B, 84(24):245441, December 2011. 
[124] Y.-W. Tan, Y. Zhang, K. Bolotin, Y. Zhao, S. Adam, E. H. Hwang, S. Das Sarma, H. L. Stormer, and P. Kim. Measurement of scattering rate and minimum conductivity in graphene. Phys. Rev. Lett., 99:246803, Dec 2007 .

[125] J.-H. Chen, C. Jang, S. Adam, M. S. Fuhrer, E. D. Williams, and M. Ishigami. Charged-impurity scattering in graphene. 4(5):377-381.

[126] Dong Sun, Charles Divin, Momchil Mihnev, Torben Winzer, Ermin Malic, Andreas Knorr, John E Sipe, Claire Berger, Walt A de Heer, Phillip N First, and Theodore B Norris. Current relaxation due to hot carrier scattering in graphene. New Journal of Physics, 14(10):105012, October 2012.

[127] Jens Christian Johannsen, Søren Ulstrup, Federico Cilento, Alberto Crepaldi, Michele Zacchigna, Cephise Cacho, I. C. Edmond Turcu, Emma Springate, Felix Fromm, Christian Raidel, Thomas Seyller, Fulvio Parmigiani, Marco Grioni, and Philip Hofmann. Direct view of hot carrier dynamics in graphene. Physical Review Letters, 111(2):027403, July 2013.

[128] Jens Christian Johannsen, Søren Ulstrup, Alberto Crepaldi, Federico Cilento, Michele Zacchigna, Jill A. Miwa, Cephise Cacho, Richard T. Chapman, Emma Springate, Felix Fromm, Christian Raidel, Thomas Seyller, Phil D. C. King, Fulvio Parmigiani, Marco Grioni, and Philip Hofmann. Tunable carrier multiplication and cooling in graphene. Nano Letters, 15(1):326-331, 2015. PMID: 25458168.

[129] Justin C. W. Song, Mark S. Rudner, Charles M. Marcus, and Leonid S. Levitov. Hot carrier transport and photocurrent response in graphene. Nano Letters, 11(11):4688-4692, November 2011.

[130] Torben Winzer and Ermin Malić. Impact of auger processes on carrier dynamics in graphene. Physical Review B, 85(24):241404, June 2012.

[131] Luca Pirro, Anuj Girdhar, Yusuf Leblebici, and Jean-Pierre Leburton. Impact ionization and carrier multiplication in graphene. Journal of Applied Physics, 112(9):093707-093707-9, November 2012.

[132] D. M. Basko. Effect of anisotropic band curvature on carrier multiplication in graphene. Physical Review B, 87(16):165437, April 2013.

[133] T. Plötzing, T. Winzer, E. Malic, D. Neumaier, Knorr A., and H. Kurz. Experimental verification of carrier multiplication in graphene. Nano Lett., 14:5371, 2014. 
[134] F. Wendler, A. Knorr, and E. Malic. Carrier multiplication in graphene under landau quantization. Nature Commun., 5:3703, 2014.

[135] I. Gierz, F. Calegari, S. Aeschlimann, M. Chavez Cervantes, C. Cacho, R. T. Chapman, E. Springate, S. Link, U. Starke, C. R. Ast, and A. Cavalleri. Tracking primary thermalization events in graphene with photoemission at extreme timescales. ArXiv:1506.00120, 2015.

[136] Ermin Malic, Torben Winzer, and Andreas Knorr. Efficient orientational carrier relaxation in optically excited graphene. Applied Physics Letters, 101(21):213110-213110-3, November 2012.

[137] Faris Kadi, Torben Winzer, Andreas Knorr, and Ermin Malic. Doping dependence of carrier dynamics in graphene. submitted, 2015. 
